LA-13972-MS

Approved for public release

distribution is unlimited.

Geochemical Data on Waters, Gases,

Scales, and Rocks from the Dixie

Valley Region, Nevada (1996-1999)

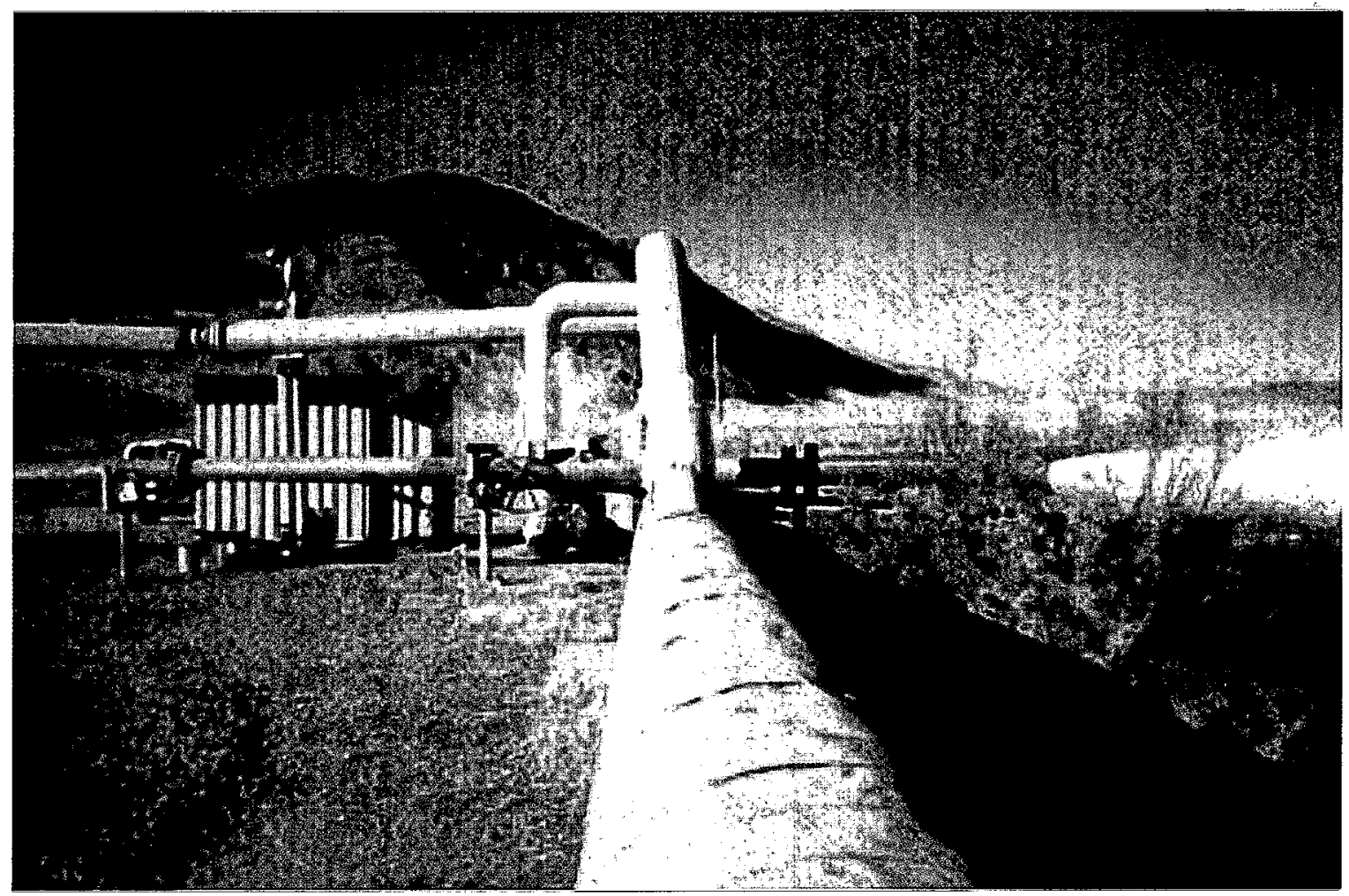

Fos Alamos

Dide valler

SMU GEOTHERMAL LAB OST $1-12$ 


\section{DISCLAIMER}

This report was prepared as an account of work sponsored by an agency of the United States Government. Neither the United States Government nor any agency Thereof, nor any of their employees, makes any warranty, express or implied, or assumes any legal liability or responsibility for the accuracy, completeness, or usefulness of any information, apparatus, product, or process disclosed, or represents that its use would not infringe privately owned rights. Reference herein to any specific commercial product, process, or service by trade name, trademark, manufacturer, or otherwise does not necessarily constitute or imply its endorsement, recommendation, or favoring by the United States Government or any agency thereof. The views and opinions of authors expressed herein do not necessarily state or reflect those of the United States Government or any agency thereof. 


\section{DISCLAIMER}

Portions of this document may be illegible in electronic image products. Images are produced from the best available original document. 
Cover Photo: Photo looking NNE of 37-33 production well and flow lines from the northern production zone, Dixie Valley geothermal field, Nevada. Geothermal brine and steam are being vented into a holding pond on the right side of photo. The Stillwater Range towers above Dixie Valley in the left background. The break in the slope between the range and the valley marks the approximate position of the Stillwater fault zone (photo by F. Goff).

Los Alamos National Laboratory, an affirmative action/equal opportunity employer, is operated by the University of California for the United States Department of Energy under contract W-7405-ENG-36.

This report was prepared as an account of work sponsored by an agency of the United States Government. Neither the Regents of the University of California, the United States Government nor any agency thereof, nor any of their employees make any warranty, express or implied, or assume any legal liability or responsibility for the accuracy, completeness, or usefulness of any information, apparatus, product, or process disclosed, or represent that its use would not infringe privately owned rights. Reference herein to any specific commercial product, process, or service by trade name, trademark, manufacturer, or otherwise does not necessarily constitute or imply its endorsement, recommendation, or favoring by the Regents of the University of California, the United States Government, or any agency thereof. The views and opinions of authors expressed herein do not necessarily state or reflect those of the Regents of the University of California, the United States Government, or any agency thereof. Los Alamos National Laboratory strongly supports academic freedom and a researcher's right to publish; as an institution, however, the Laboratory does not endorse the viewpoint of a publication or guarantee its technical correctness. 
LA-13972-MS

Issued: August 2002

Geochemical Data on Waters, Gases, Scales, and

Rocks from the Dixie Valley Region, Nevada

(1996-1999)

Fraser Goff

Deborah Bergfeld

Cathy J. Janik*

Dale Counce

Mike Murrell

* U.S. Geological Survey, MS 910, 345 Middlefield Road, Menlo Park, CA 94025 cjanik@usgs.gov

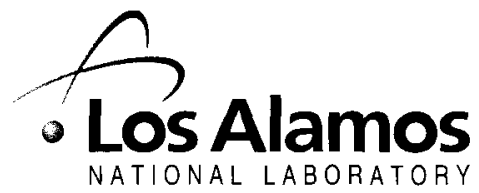




\section{Contents}

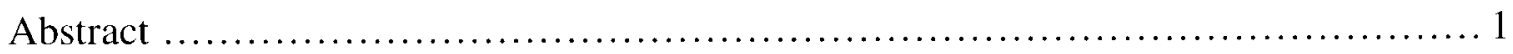

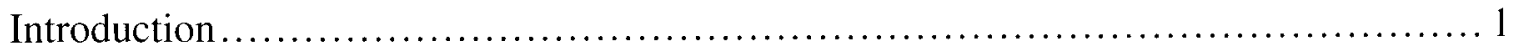

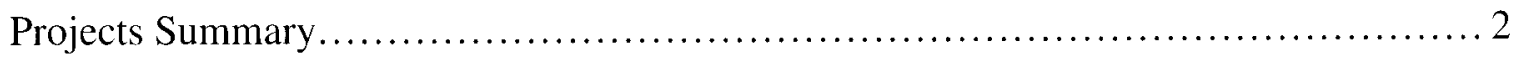

Locations And Field Parameters ............................................. 3

Sampling Methods ........................................................ 3

Analytical Methods, Results, and Calculations ................................ 6

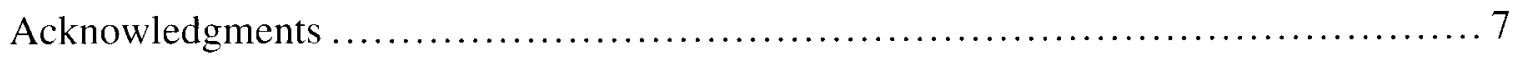

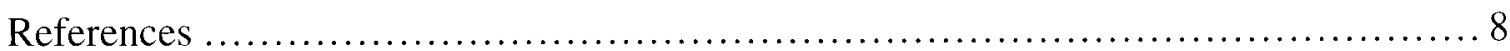

\section{Figures}

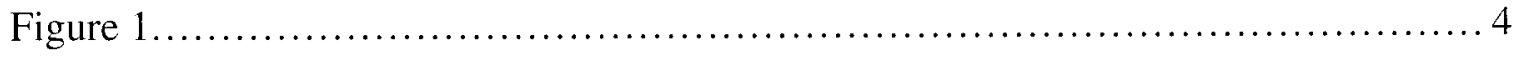

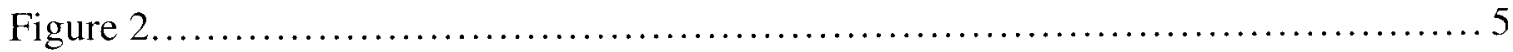

\section{Tables}

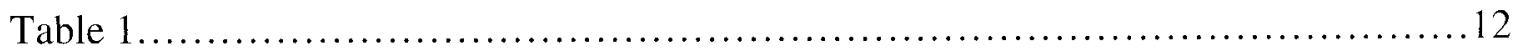

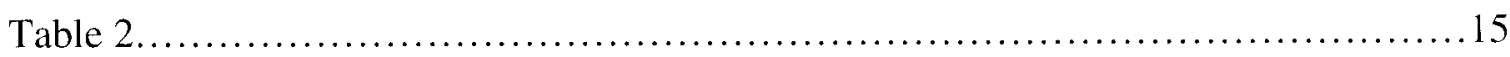

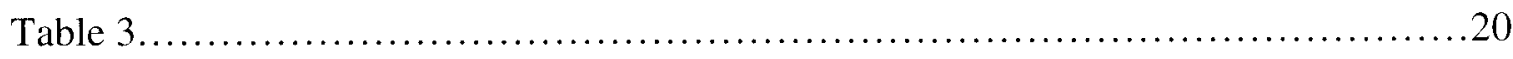

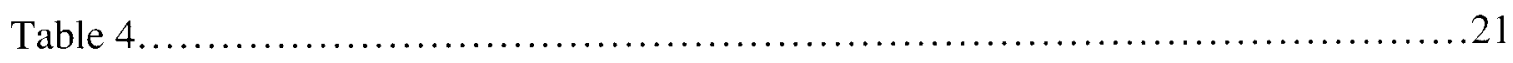

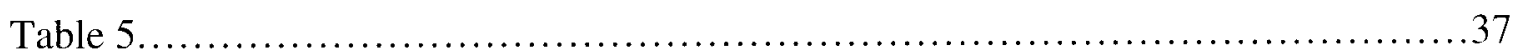

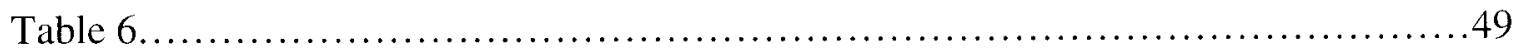

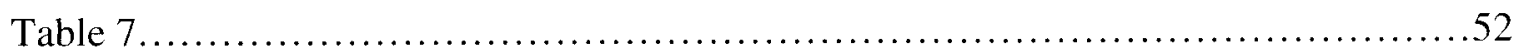

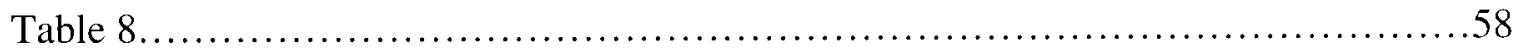

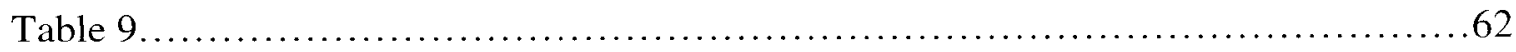

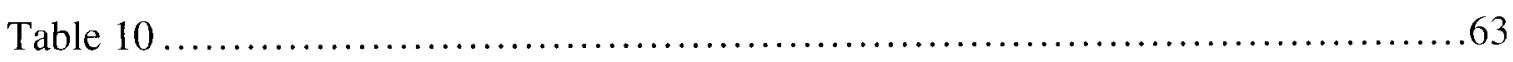

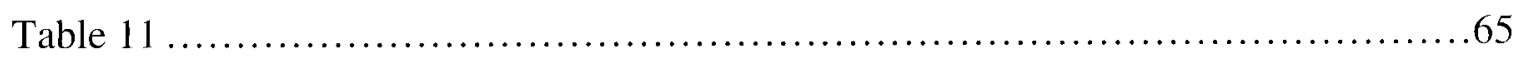

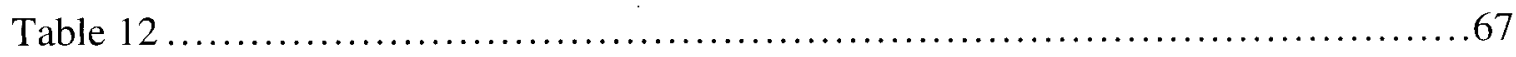

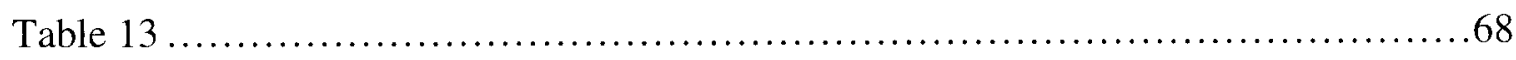

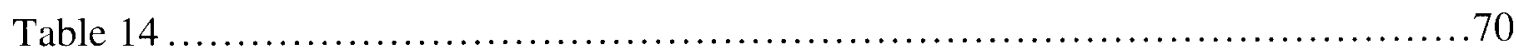

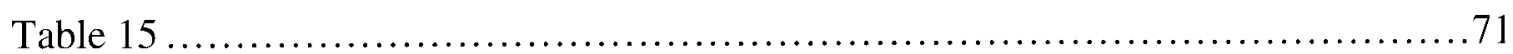




\title{
Geochemical Data on Waters, Gases, Scales, and Rocks from the Dixie Valley Region, Nevada (1996-1999)
}

\author{
by
}

Fraser Goff, Deborah Bergfeld, Cathy J. Janik,

Dale Counce, Mark Huebner, and Mike Murrell

\begin{abstract}
ABSTRAC'T
This report tabulates an extensive geochemical database on waters, gases, scales, rocks, and hot-spring deposits from the Dixie Valley region, Nevada. The samples from which the data were obtained were collected and analyzed during 1996 to 1999. These data provide useful information for ongoing and future investigations on geothermal energy, volcanism, ore deposits, environmental issues, and groundwater quality in this region.
\end{abstract}

\section{INTRODUCTION}

The Dixie Valley geothermal system is located roughly $160 \mathrm{~km}$ northeast of Fallon in west central Nevada and supports a 62-MWe double-flash power plant that became operational in 1988. Located in the Basin and Range tectonic province, Dixie Valley trends north-northeast and is $120 \mathrm{~km}$ long by about $20 \mathrm{~km}$ wide. It is bounded by the Stillwater Range on the west and by the Clan Alpine Range to the east (Waibel 1987; Honjas et al. 1997; Lutz et al. 1997). Geothermal fluids are produced from the subsurface extension of the normal Stillwater fault zone and associated fractured rocks at depths of 3,050 to $2,800 \mathrm{~m}$. The fault zone architecture is considered to be complex (Caine et al. 1996). The Dixie Valley geothermal system displays fault and fracture permeability typical of the Basin and Range. Convective heat flow in the geothermal system exceeds $300 \mathrm{~mW} / \mathrm{m}^{2}$, and conductive temperature gradients range from $100^{\circ} \mathrm{C}$ to $>200^{\circ} \mathrm{C} / \mathrm{km}$ (Williams et al. 1997).

Producing geologic formations penetrated by geothermal wells are exposed in the Stillwater Range west of the power plant. These rocks consist of Triassic to Jurassic marine quartzite, siltstone, shale, and volcaniclastic rocks overlain by the Humboldt Lopolith (Speed 1976), a complex of oceanic crustal rocks that includes gabbro, diorite, and basalt (Waibel 1987; Lutz 1997). The Triassic to Jurassic units have been imbricated into four similar stratigraphic packages by three thrust faults and were later intruded by Cretaceous granodiorite. Uplift and erosion exposed these older rocks by the mid-Tertiary during which an extensive complex of Oligocene ignimbrites was emplaced throughout the region. The Miocene Table Mountain Basalt rests on earlier rocks in the subsurface of Dixie Valley and in the bordering ranges. Within Dixie Valley, the basalt is found at $1,280 \mathrm{~m}$ below sea level and is overlain by a variety of late Tertiary basin-fill deposits. The mouths of all major canyons are now filled by alluvial fans, and the axis of Dixie Valley is occupied by alluvium, playa deposits, and the Humboldt Salt Marsh. 
Extensive alteration from previous hydrothermal activity and present geothermal fluids affects most geologic units, particularly along range front faults. A series of active and dead hot springs and fumaroles is sporadically distributed along the Stillwater fault from a zone a few kilometers north of the Dixie Valley power plant (Senator fumarole group) to a point about $20 \mathrm{k}$ southwest of the power plant (Dixie hot springs). Additional hot and mineral fluids discharge as springs or occur as shallow aquifers throughout the Dixie Valley region.

The authors have been engaged in a series of geothermal investigations in the Dixie Valley region since October 1996 (Bruton et al. 1997; Goff et al. 1998; Kennedy et al. 1999; Nimz et al. 1999; Goff and Janik 2000; Bergfeld 2001; Bergfeld et al. 1998, 2001; Stamates 2001). During these investigations, large quantities of unpublished chemical and isotopic data on waters, gases, rocks, and hot spring deposits have accumulated. Because this data may be useful to other research groups and interested regional stakeholders, we are releasing this information into the public domain. This report contains the locations, field measurements, and analytical results of these accumulated data. No attempt is made herein to interpret the scientific meaning of these data.

\section{PROJECTS SUMMARY}

The following geothermal collaborations were responsible for production of the data included in this report:

1. A study of rock-water interaction, corrosion, and scaling of production/injection horizons, production wells, and injection wells with C. Bruton, Lawrence Livermore National Laboratory (LLNL) and J. Moore (Energy and Geoscience Institute).

2. A study of temporal and spatial stable isotope variations in production fluids of the Dixie Valley geothermal reservoir with C. J. Janik, U.S. Geological Survey (USGS).

3. A study of recharge sources and fluid ages with respect to the Dixie Valley region and the Dixie Valley geothermal system with G. Nimz (LLNL) and C. J. Janik (USGS).

4. A study of the distribution and flux of anomalous $\mathrm{CO}_{2}$ and elevated steam discharge at the "dead zone" on the north edge of the Dixie Valley geothermal system with D. Bergfeld, Los Alamos National Laboratory (LANL) and C. J. Janik (USGS). This project was one of those contained in Bergfeld's Ph.D. thesis.

5. An evaluation of gold, mercury, and other trace metals contents in Dixie Valley production fluids and scales with S. Johnson, Oxbow Geothermal Company.

6. An investigation of the geology and age of selected travertine and sinter deposits within and along the flanks of Dixie Valley with M. Murrell (LANL) and C. J. Janik (USGS).

7. An investigation examining the tritium relations of Dixie Valley geothermal and regional fluids with M. Stamates and L. Shevenell (University of Nevada- Reno). This project made up a portion of Stamates' M.S. thesis. 


\section{LOCATIONS AND FIELD PARAMETERS}

Latitude, longitude, and elevation of the sampling sites appear in Table 1, and the sites are keyed to map numbers on the regional and detailed maps of Figures 1 and 2. Table 1 also provides the name of the 1:100,000 topographic quadrangle and the lithology of each sampling site. Field parameters for the sampling sites appear in Table 2. All field and analytical data are keyed to sample numbers that are listed as initial entries in most of the tables. Temperatures were measured with thermocouples and digital thermometers, or occasionally, from gauges on geothermal wells. Pressures were obtained from gauges on geothermal wells, from a portable digital pressure gauge that was piped into our sampling equipment, or from power plant data. Steam fractions (y) were provided to us by the operator of the geothermal field. The field $\mathrm{pH}$ of produced fluids, injection fluids, and power plant fluids was usually measured with a $\mathrm{pH}$ electrode. The field $\mathrm{pH}$ of background waters was measured with $\mathrm{pH}$-sensitive papers. Field alkalinity and conductivity of produced fluids, injection fluids, and power plant fluids were determined by $\mathrm{pH}$ titration and with a portable conductivity meter, respectively. The field Eh was obtained with a field portable electrode. Flow rates of wells, power plant fluids, and injection lines were provided to us by the operator of the geothermal field. Flow rates of background fluids were generally measured with a bucket or beaker and a stopwatch.

\section{SAMPLING METHODS}

Water Samples: Field procedures for sampling waters have been described in detail by Trujillo et al. (1987), Werner et al. (1997), and Goff and McMurtry (2000). Generally, four basic samples are collected at each water collection site: (1) a 125-ml plastic bottle of filtered $(0.45 \mathrm{~mm})$, unacidified water for anions, (2) a $125-\mathrm{ml}$ plastic bottle of filtered $(0.45 \mathrm{~mm})$ water, acidified to $\mathrm{pH} \leq 2$ with spectrographically pure, concentrated $\mathrm{HNO}_{3}$ for cations, silica, and trace metals, (3) a 30-ml glass bottle of raw water for deuterium and oxygen-18 isotope measurements, and (4) a 500-ml glass or plastic bottle of raw water for tritium measurements. Sampling for trace metals analysis followed a clean-hands protocol in order to avoid the introduction of contaminants. Samples were collected in precleaned, acid-washed (nitric acid) bottles and preserved with trace-metal grade reagents.

\footnotetext{
Aluminum and Silica Samples of Production, Injection, and Other Waters: Special samples were collected for total and ionized aluminum to allow for thermodynamic modeling of mineral phases in equilibrium or nonequilibrium with the production and injection well fluids (Bruton et al. 1997; Gallup 1998). The filtered, acidified sample described above was used for total aluminum analyses. Ionized aluminum was extracted from $500 \mathrm{ml}$ of filtered sample using an oxine-MIBK method modified from Barnes (1975). Both 0.2- and 0.45- $\mu$ filters were used on the samples before extraction proceeded. Final extracts were stored in silica-glass vials in coolers and refrigerators before analysis. In early 1999 C. Bruton (LLNL) indicated that the analyzed values for ionized aluminum determined previously were probably too low, due possibly to coagulation of colloid particles and loss of aluminum during filtering. Thus, 1999 samples were determined on unfiltered oxine-MIBK extracts.
} 


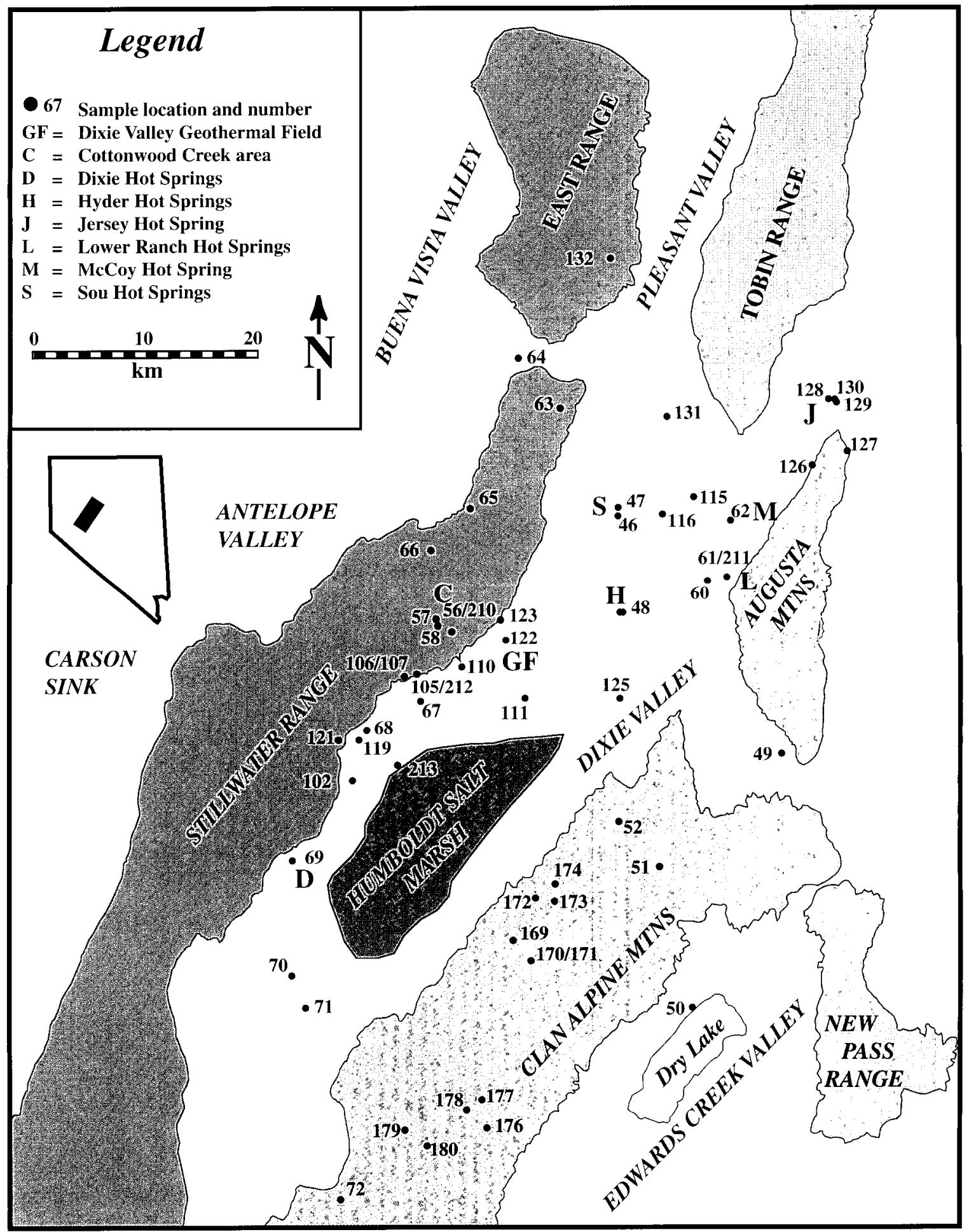

Figure 1. Regional map of the Dixie Valley region, Nevada, showing the locations of samples (Table 1). 


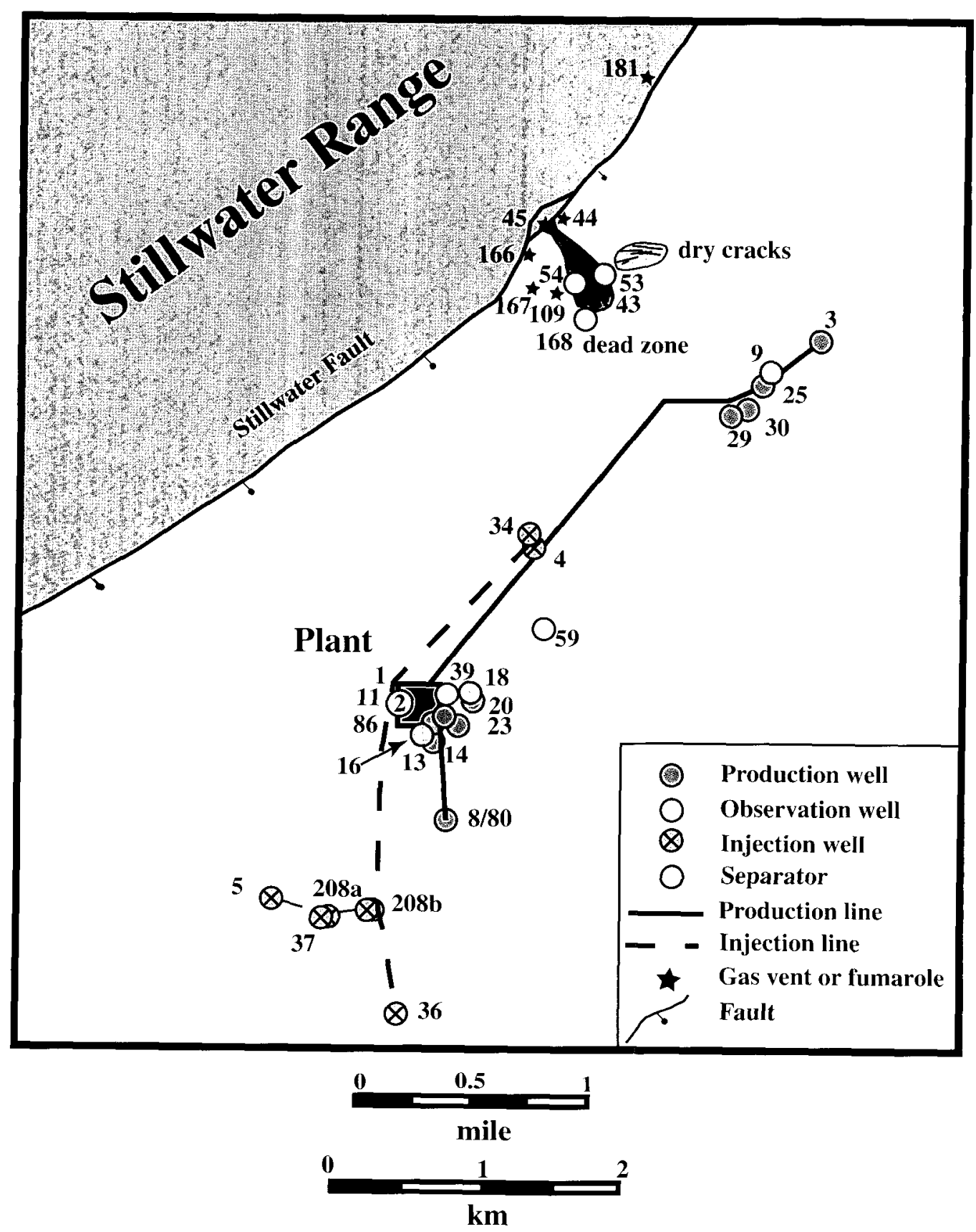

Figure 2. Map of west central Dixie Valley in the vicinity of the geothermal power plant showing locations of production, injection, shallow observation, and water supply wells, the locations of fumaroles along the Stillwater fault zone, and the location of the "dead" zone (Bergfeld et al. 2001). North is straight up on this figure. 
Silica samples of initially hot or supersaturated fluids (such as those from production and injection wells, from power plant fluids, from other geothermal wells, and from hot springs) were obtained by pipetting $5 \mathrm{ml}$ of fresh sample into $60 \mathrm{ml}$ plastic vials containing $30 \mathrm{ml}$ of deionized, silica-free water. Silica from cold features was determined on the filtered, acidified samples described above.

Additional Water Samples: Additional samples were collected for other kinds of analyses but were not collected at each sampling point or for all projects described above. For sulfide analysis, $5 \mathrm{ml}$ raw water sample was pipeted into a $15 \mathrm{ml}$ plastic bottle containing $5 \mathrm{ml}$ of sulfide antioxidant buffer solution. Gold samples were collected by filling a 125- $\mathrm{ml}$ prewashed glass bottle with filtered water and adding $2 \mathrm{ml}$ of aqua regia (a 3:1 mixture of trace-metal-grade $\mathrm{HCl}$ and $\mathrm{HNO}_{3}$ ). Samples for carbon-13 analysis of dissolved inorganic carbon were collected by filling a $125-$ to $250-\mathrm{ml}$ glass bottle with raw water and adding a saturated solution (in most cases $10 \mathrm{ml}$ ) of $\mathrm{SrCl}_{2}$ in $\mathrm{NH}_{4} \mathrm{OH}$.

Gases: Gas samples were obtained at fumaroles, gas vents, gaseous springs and gas-rich wells with funnels, pipes, tubing, and fittings as necessary. Gases were collected in 300$\mathrm{ml}$ double-port, caustic-gas bottles as described by Trujillo et al. (1987), Fahlquist and Janik (1992), and Goff and McMurtry (2000). Caustic-gas bottles are prepared in the laboratory before sampling by adding roughly $100 \mathrm{ml}$ of 4N $\mathrm{NaOH}$ solution (bicarbonate purged) to the bottle and pumping the remaining head space of the bottle to vacuum. Samples collected in caustic bottles can be used for bulk gas analysis and analysis of $\delta^{13} \mathrm{C}-\mathrm{CO}_{2}, \delta^{13} \mathrm{C}-\mathrm{CH}_{4}$, and other isotopic constituents.

Scales, Rocks, and Hot Springs Deposits: Samples of test bed scales were provided by J. Moore $(E G \& G)$. Samples of production and injection well scales were donated by $S$. Johnson (Oxbow Power Co.). Samples of representative volcanic, plutonic, metamorphic, and sedimentary rocks from the Dixie Valley region were identified using geologic and paleomagnetic maps and reports (Speed 1976; Hudson 1988; Hudson and Geissman 1984, 1991; Plank et al. 1999). These samples were analyzed for various isotopes using standard methods by several contract laboratories. Samples of hot spring deposits from the "dead" travertine in Cottonwood Canyon and the Lower Ranch sinter-travertine in northeastern Dixie Valley were collected for U-series dating methods. All scale and rock samples were collected in cloth or plastic bags for later chemical and isotopic analysis.

\section{ANALYTICAL METHODS, RESULTS, AND CALCULATIONS}

Waters: Major and trace element chemical analyses of waters were determined by D. Counce at Los Alamos National Laboratory using methods listed in Table 3 (Janik et al. 1999; Goff et al. 2001). Results of the analyses are given in Tables 4 and 5. Total aluminum was determined by inductively coupled plasma (ICP) spectroscopy on the acidified sample described above. Ionized aluminum was determined on methyl isobutyl ketone (MIBK) extracts described above using graphite furnace atomic adsorption (GFAA) spectroscopy. The aluminum results can be compared on Table 6. Isotope analyses of $\delta \mathrm{D}-\mathrm{H}_{2} \mathrm{O}, \delta^{18} \mathrm{O}-\mathrm{H}_{2} \mathrm{O}, \delta^{13} \mathrm{C}-\mathrm{HCO}_{3}$, and $\delta^{18} \mathrm{O}-\mathrm{SO}_{4}$ were determined by standard methods at various laboratories listed in Table 7. Tritium measurements were obtained primarily from the University of Miami. All isotope results on water samples are reported in Table 7. 
Gases: Bulk gas analyses (Table 8) were obtained from either the U.S. Geological Survey by C. J. Janik or the EES-6 geochemistry lab at Los Alamos National Laboratory by $\mathrm{D}$. Counce using gas methods listed in Table 3. Carbon-13 analyses of $\mathrm{CO}_{2}$ were determined using standard methods at a variety of laboratories as listed in Table 8 . Trace metals analyses of the caustic solutions from selected gas samples are listed in Table 9. These analyses used procedures for liquid samples listed in Table 3, and the results are adjusted for the density of the caustic solution.

Chemical Geothermometers: Results for the sulfate oxygen isotope geothermometer are listed in Table 7. Note that this geothermometer has many limitations due to brine flash, reequilibration, evaporation, and mixing of different fluids. Gas geothermometers that are used to estimate subsurface geothermal reservoir temperatures are listed for all gas samples in Table 9. Reconstructed chemical compositions of flashed brines are given in Table 10. Geothermometer calculations of the reconstructed brines are shown in Table 11. Geothermometer calculations of thermal and mineral springs and wells are listed in Table 12. All calculations use standard geothermometers that are referenced at the bottom of the various tables. Calculations were performed on a personal computer using the code of Urbani (1986).

Scales: About $0.25 \mathrm{~g}$ of dried scale and test-bed precipitates were mixed with a cocktail consisting of $2.0 \mathrm{ml} \mathrm{HNO}, 3.5 \mathrm{ml} \mathrm{HCl}$, and $1.5 \mathrm{ml} \mathrm{HF}$, heated in a microwave oven for about 10 minutes, and the resulting solution adjusted to $50 \mathrm{ml}$ with deionized water. Analyses of selected metals by methods described in Table 2 appear in Table 13. This method extracts easily soluble metallic minerals and colloid particles from the scales without dissolving all the rock fragments, sand grains, and silt that may be in some samples.

Rocks: Rock samples were cleaned of debris and sent to contract laboratories for isotopic analyses as listed in Table 14. Rubidium and strontium concentrations were analyzed at LANL for a group of strontium isotope samples collected in 1998. The rubidium and strontium concentrations for strontium isotope samples collected for an earlier project were analyzed at the University of New Mexico (UNM).

Hot Spring Deposits: Two areas of hot-spring deposits were examined in detail and sampled for U-series radiometric dating: the Cottonwood Canyon travertine and the Lower Ranch mixed travertine and sinter. Samples were cleaned, crushed, and hand picked to obtain 5 to $20 \mathrm{~g}$ of pure carbonate and silica (opal and chalcedony). Samples were further processed in highly purified acids and analyzed by mass spectrometry according to procedures described in Edwards et al. (1997) and Pickett and Murrell (1997).

\section{ACKNOWLEDGMENTS}

Collection of the various samples and publication of this report was made possible by $\mathrm{M}$. Reed and J. Renner of the U.S. Department of Energy, Office of Geothermal Technologies. C. J. Janik was also funded by the U.S. Geological Survey Volcano Hazards Program. Grants from the Geological Society of America supported some of the field work for D. Bergfeld. Stu Johnson and Dick Benoit (Oxbow Power Co.) provided valuable information and lots of muscle at the Dixie Valley geothermal system. Jerry Kelly (DJ Ranch) gave us free access to the Lower Ranch hot springs. J. Brinkerhoff cheerfully allowed us to sample his wells in northern Dixie Valley. The manuscript was reviewed by J. Heikoop (LANL), and Mable Amador provided the final editing. 


\section{REFERENCES}

Barnes, R.B., 1975, The determination of specific forms of aluminum in natural water. Chemical Geology, v. 15, p. 177-191.

Bergfeld, D., 2001, Geothermal systems and $\mathrm{CO}_{2}$ degassing: The Geysers-Clear Lake, and Dixie Valley regions of California and Nevada. Ph.D. thesis, University of New Mexico, Albuquerque, $123 \mathrm{pp}$.

Bergfeld, D., Goff, F.E., and Janik, C.J., 2001, Elevated carbon dioxide flux at the Dixie Valley geothermal field, Nevada; relations between surface phenomena and the geothermal reservoir, Chemical Geology, v. 177, p. 43-66.

Bergfeld, D., Goff, F.E., and Janik, C.J., Johnson, S., 1998, $\mathrm{CO}_{2}$ flux measurements across portions of the Dixie Valley geothermal system, Nevada. Geothermal Resources Council Transactions, v. 22, p. 107-111.

Bruton, C., Counce, D., Bergfeld, D., Goff, F., Johnson, S., Moore, J., and Nimz, G., 1997, Preliminary investigation of scale formation and fluid chemistry at the Dixie Valley geothermal field, Nevada. Geothermal Resources Council Transactions, v. 21, p. $157-164$.

Caine, J., Evans, J., and Forster, C.B., 1996, Fault zone architecture and permeability structure. Geology, v. 24, p. 1025-1028.

D'Amore, F., and Panichi, C., 1980, Evaluation of deep temperatures of hydrothermal systems by a new gas geothermometer. Geochimica Cosmochimica Acta, v. 44, p. 549-556.

Edwards, R.L., Cheng, H., Murrell, M.T., and Goldstein, S.J., 1997, Protactinium-231 dating of carbonates by thermal ionization mass spectrometry: Implications for quaternary climate change. Science, v. 276, p. 782-786.

Fahlquist, L., and Janik, C.J., 1992, Procedures for collecting and analyzing gas samples from geothermal systems. U.S. Geological Survey, Open-File Report 92-211, 19 pp.

Fouillac, C., and Michard, G., 1981, Sodium-lithium ratios in water applied to geothermometry of geothermal reservoirs. Geothermics, v. 10, p. 55-70.

Fournier, R.O., 1981, Application of water geochemistry to geothermal exploration and reservoir engineering, in (Rybach, L., and Muffler, L.J.P., eds.) Geothermal Systems, Principals and Case Histories. Wiley, NY, p. 109-143.

Fournier, R.O., and Potter, R.W., 1979, Magnesium correction to the Na-K-Ca chemical geothermometer. Geochimica Cosmochimica Acta, v. 43, p. 1543-1550.

Fournier, R.O., and Truesdell, A.H., 1973, An empirical Na-K-Ca geothermometer for natural waters. Geochimica Cosmochimica Acta, v. 37, p. 1255-1275.

Gallup, D.L., 1998, Aluminum silicate scale formation and inhibition (2): Scale solubilities and laboratory and field inhibition tests. Geothermics, v. 27, p. 485-501. 
Giggenbach, W.F., 1986, Graphical techniques for the evaluation of water/rock equilibration conditions by use of $\mathrm{Na}, \mathrm{K}, \mathrm{Mg}$, and $\mathrm{Ca}$ contents of discharge waters. Proceedings 8th N.Z. Geothermal Workshop, Auckland, N.Z., University of Auckland, p. $37-43$.

Giggenbach, W.F., 1992, Chemical techniques in geothermal exploration.

UNITAR/UNDP, Rome, Italy, p. 119-144.

Goff, F., and Janik, C.J., 2000, Geothermal Systems, in (H. Sigurdsson, ed.)

Encyclopedia of Volcanoes. Academic Press, San Diego, p. 817-834.

Goff, F., and McMurtry, G.M., 2000, Tritium and stable isotopes of magmatic waters. Journal of Volcanology and Geothermal Research, v. 97, p. 347-396.

Goff, F., Hulen, J., Adams, A., Trujillo, P., Counce, D., and Evans, W.C., 1994, Geothermal characteristics of some oil field waters in the Great Basin, Nevada, in (Schalla, R.A., and Johnson, E.H., eds.) Oil Fields of the Great Basin. Nevada Petroleum Society, Reno, Nevada, p. 93-106.

Goff. F., Janik, C.J., Bergfeld, D., Counce, D., Bruton, C., and Nimz, G., 1998, Geothermal chemistry/exploration investigations at Dixie Valley, Nevada. U.S. Department of Energy, Geothermal Program Review, Berkeley, California, 7 pp.

Goff, F., Bergfeld, D., Janik, C.J., Counce, D., and Stimac, J.A., 2001, Geochemical data on waters, gases, rocks, and sediments from The Geysers-Clear Lake region, California (1991-2000). Los Alamos National Laboratory Report LA-13882-MS, 40 pp.

Goguel, R., 1976, Thermal water transport of some major rock constituents at Wairakei. New Zealand Journal of Science, v. 19, p. 359-368.

Goguel, R., 1988, Ultratrace metal analysis of New Zealand geothermal waters by ICPMS. Proceedings of the New Zealand Trace elements Group Conference, 30 Nov - 2 Dec, 1988, Lincoln College, Canterbury, NZ, p. 263-270.

Honjas, W., Pullammanappillil, S.K., Lettis, W., Plank, G.L., Louie, J., and Schweichert, R., 1997, Predicting subsurface structure within the Dixie Valley geothermal field, Dixie Valley, Nevada, using a non-linear optimization scheme. Geothermal Resources Council Bulletin, February, pp. 45-52.

Hudson, M.R., 1988, Paleomagnetic and structural evidence bearing on the tectonic history of a region surrounding Dixie Valley, west-central Nevada. Ph.D. dissertation, Colorado School of Mines, Golden, Colorado, $352 \mathrm{pp}$.

Hudson, M.R., and Geissman, J.Wm., 1984, Preliminary paleomagnetic data from the Jurassic Humboldt Lopolith, west-central Nevada: Evidence for thrust belt rotation in the Fencemaker allochthon. Geophysical Research Letters, v. I., p. 828-831.

Hudson, M.R., and Geissman, J.W., 1991, Paleomagnetic evidence for the age and extent of middle Tertiary counterclockwise rotation, Dixie Valley region, west central Nevada. Journal of Geophysical Research, v. 96, p. 3979-4006. 
Hulen, J. B., Goff, F., Bereskin, S. R., and Bortz, L. C., 1994, Geology and geothermal origin of Grant Canyon and Bacon Flat oil fields, Railroad Valley, Nevada: American Association of Petroleum Geologists Bulletin, v. 78, p. 596-623.

Janik, C.J., Goff, F., Sorey, M., Rytuba, J., Counce, D., Colvard, E., Huebner, M., White, L.D., and Foster, A., 1999, Physical, chemical, and isotopic data for samples from the Anderson Springs area, Lake County, California, 1998-1999. U.S. Geological Survey Open-File Report 99-585, 27 pp.

Kennedy, B.M., Janik, C., Benoit, D., and Shuster, D., 1999, Natural geochemical tracers for injectate fluids at Dixie Valley. Proceedings, 24th Workshop on Geothermal Reservoir Engineering, Stanford University Report SGP-TR-162, Stanford, California, p. $108-115$.

Kharaka, Y. and Mariner, R., 1989, Chemical geothermometers and their application to formation waters from sedimentary basins, in (Naeser, N., and McCulloh, T., eds.) Thermal History of Sedimentary Basins: Methods and Case Histories. Springer-Verlag, Berlin, p. 99-177.

Lutz, S., Moore, J., and Benoit, D., 1997, Geologic framework of Jurassic reservoir rocks in the Dixie Valley geothermal field, Nevada: Implications from hydrothermal alteration and stratigraphy. Proceedings, 22nd Workshop on Geothermal Reservoir Engineering, Stanford University Report SGP-TR-155, Stanford, California, p. 131-139.

McKenzie, W.F., and Truesdell, A.H., 1977, Geothermal reservoir temperatures estimated from the oxygen isotope compositions of dissolved sulfate and water from hot springs and shallow drillholes. Geothermics, v. 5, p. 51-61.

Nimz, G., Janik, C., Goff, F., Dunlap, C., Huebner, M., Counce, D., and Johnson, S., 1999, Regional hydrology of the Dixie Valley geothermal field, Nevada: preliminary interpretations of chemical and isotopic data. Geothermal Resources Council Transactions, v. 23, p. 333-338.

Norman, D., and Bernhardt, C., 1981, Assessment of geothermal reservoirs by analysis of gases in thermal waters. Final Technical Report, New Mexico Energy Institute, New Mexico State University, Las Cruces, 130 pp.

Picket, D.A., and Murrell, M.T., 1997, Observations of ${ }^{231} \mathrm{~Pa} /{ }^{235} \mathrm{U}$ disequilibrium in volcanic rocks. Earth and Planetary Science Letters, v. 148, p. 259-271.

Plank, G., Schweickert, R., Benoit, D., and Simmons, A., 1999, Influence of fault surface geometry on the location of the Dixie Valley geothermal area, Dixie Valley, Nevada. Proceedings, 24th Workshop on Geothermal Reservoir Engineering, Stanford University Report SGP-TR-162, Stanford, California, 8 pp.

Speed, R.C., 1976, Geologic map of the Humboldt Lopolith. Geological Society of America Map Chart Series MC-14, 1:81050 scale, 1 sheet.

Stamates, M., 2001, Evaluation of injection effects on the Dixie Valley, Nevada, geothermal reservoir through the use of geochemical data. M.S. thesis, University of Nevada, Reno, 198 pp. 
Trujillo, P., Counce, D., Grigsby, C., Goff, F., and Shevenell, L., 1987, Chemical analysis and sampling techniques for geothermal fluids and gases at the Fenton Hill laboratory. Los Alamos National Laboratory, Report LA-11006-MS, 84 pp.

Urbani, F., 1986, GTHERM, a spreadsheet and graphic setup for geothermal exploration. Unpublished report, Los Alamos National Laboratory, 103 pp.

Waibel, A.F., 1987, An overview of the geology and secondary mineralogy of the high temperature geothermal system in Dixie Valley, Nevada. Geothermal Resources Council Transactions, v. 11, p. 479-486.

Werner, C., Janik, C.J., Goff, F., Counce, D., Johnson, L., Siebe, C., Delgado, H., Williams, S.N., and Fischer, T.P., 1997, Geochemistry of summit fumarole vapors and flanking thermal/mineral waters at Popocatépetl Volcano, Mexico. Los Alamos National Laboratory, Report LA-13289-MS, 33 pp.

Williams, C., Sass, J., and Grubb, F., 1997, Thermal signature of subsurface fluid flow in the Dixie Valley geothermal field, Nevada. Proceedings, 22nd Workshop on Geothermal Reservoir Engineering, Stanford University Report SGP-TR-155, Stanford, California, Jan. 27-29, 8 pp. 
Table 1: Sample Types, Locations, Elevations, and Surface Rocks of Fluid Samples Collected for the Dixie Valley Geothermal Project, Nevada.

\begin{tabular}{|c|c|c|c|c|c|c|c|c|}
\hline Name or Description & $\begin{array}{c}\text { Map" } \\
\text { Number }\end{array}$ & $\begin{array}{l}\text { Map } \\
\text { Figure }\end{array}$ & $\begin{array}{l}\text { Latitude } \\
( \pm 25 \mathrm{~m})\end{array}$ & $\begin{array}{l}\text { Longitude } \\
( \pm 25 \mathrm{~m})\end{array}$ & $\begin{array}{l}\text { Elevation } \\
\qquad( \pm \mathbf{3 ~ m})\end{array}$ & $\begin{array}{c}\text { USGS 1:100,000 } \\
\text { Metric Quadrangle }\end{array}$ & Surface Rocks & Comments \\
\hline \multicolumn{9}{|l|}{ Production Wells } \\
\hline $27-33$ Well & 25 & 2 & 39.9869 & 117.8307 & 1050 & Edwards Creek Valley & Alluvium & Bottoms in quartzite/granodiorite \\
\hline 28-33 Well & 30 & 2 & 39.9852 & 117.8318 & 1057 & Edwards Creek Valley & Alluvium & Bottoms in quartzite/granodiorite \\
\hline 37-33 Well & 29 & 2 & 39.9861 & 117.8312 & 1055 & Edwards Creek Valley & Alluvium & Added in July 1997; quartzite/granodiorite \\
\hline 45-33 Well Archived & 3 & 2 & 39.9897 & 117.8260 & 1051 & Edwards Creek Valley & Alluvium & Bottoms in quartzite/granodiorite \\
\hline 63-7 Well & 86 & 2 & 39.9650 & 117.8580 & 1059 & Edwards Creek Valley & Alluvium & Bottoms in gabbro/quartzite/granodiorite \\
\hline 73-7 Well & 11 & 2 & 39.9655 & 117.8576 & 1059 & Edwards Creek Valley & Alluvium & Bottoms in gabbro/quartzite/granodiorite \\
\hline 73B-7 Well & 23 & 2 & 39.9652 & 117.8555 & 1058 & Edwards Creek Valley & Alluvium & Added in 1995; gabbro/quartzite/granodiorite \\
\hline 74-7 Well & 14 & 2 & 39.9637 & 117.8580 & 1059 & Edwards Creek Valley & Alluvium & Bottoms in gabbro/quartzite/granodiorite \\
\hline 76-7 Well (V104 Separator) & 8 & 2 & 39.9590 & 117.8570 & 1055 & Edwards Creek Valley & Alluvium & Bottoms in gabbro/quartzite/granodiorite \\
\hline 76A-7 Well (V104 Separator) & 80 & 2 & 39.9590 & 117.8570 & 1055 & Edwards Creek Valley & Alluvium & Redrilled in July 1993 from 7,498 \\
\hline 82A-7 Well & 20 & 2 & 39.9675 & 117.8548 & 1056 & Edwards Creek Valley & Alluvium & Bottoms in gabbro/quartzite/granodiorite \\
\hline 84-7 Well & 13 & 2 & 39.9639 & 117.8582 & 1059 & Edwards Creek Valley & Alluvium & Bottoms in gabbro/quartzite/granodiorite \\
\hline V101 Separator & 9 & 2 & 39.9869 & 117.8307 & 1046 & Edwards Creek Valley & Alluvium & $27-33,28-33$, and $37-33$ wells \\
\hline V102+V103 Separator & 16 & 2 & 39.9639 & 117.8582 & 1055 & Edwards Creek Valley & Alluvium & $63-7,73-7,74-7$, and $84-7$ wells \\
\hline V105 Separator & 18 & 2 & 39.9663 & 117.8537 & 1063 & Edwards Creek Valley & Alluvium & $73 \mathrm{~B}-7$ and $82 \mathrm{~A}-7$ wells \\
\hline \multicolumn{9}{|l|}{ Injection Well/Power Plant Fluids } \\
\hline 25-5 Injection Well & 34 & 2 & 39.9774 & 117.8498 & 1052 & Edwards Creek Valley & Alluvium & \\
\hline 32-18 Injection Well & 37 & 2 & 39.9534 & 117.8629 & 1057 & Edwards Creek Valley & Alluvium & \\
\hline 41-18 Injection Well & $208 \mathrm{a}$ & 2 & 39.9535 & 117.8632 & 1055 & Edwards Creek Valley & Alluvium & \\
\hline 45-5 Injection Well & 4 & 2 & 39.9771 & 117.8494 & 1052 & Edwards Creek Valley & Alluvium & \\
\hline 52-18 Injection Well & $208 \mathrm{~b}$ & 2 & 39.9534 & 117.8629 & 1049 & Edwards Creek Valley & Alluvium & \\
\hline 65-18 Injection Well & 36 & 2 & 39.9469 & 117.8609 & 1048 & Edwards Creek Valley & Alluvium & \\
\hline Lamb 1 Injection Well & 5 & 2 & 39.9542 & 117.8713 & 1061 & Edwards Creek Valley & Alluvium & Aka SWL-] well \\
\hline Power Plant Fluids, Miscellaneous & 2 & 2 & 39.9668 & 117.8562 & 1058 & Edwards Creek Valley & Alluvium & \\
\hline \multicolumn{9}{|c|}{ Other Geothermal and On-Site Water Wells } \\
\hline Domestic Well & 1 & 2 & 39.9658 & 117.8556 & 1052 & Edwards Creek Valley & Alluvium/Alluvial Fan & Water supply well \\
\hline Goerenger Well & 39 & 2 & 39.9693 & 117.8591 & 1050 & Edwards Creek Valley & Alluvium & Water supply well \\
\hline 27-32 Well (Dick's Well) & 54 & 2 & 39.9862 & 117.8484 & 1064 & Edwards Creek Valley & Alluvial Fan & Quartizte@122m \\
\hline $32-6$ Well & 123 & 1 & 39.9984 & 117.8463 & 1115 & Edwards Creek Valley & Alluvial Fan & Observation well \\
\hline 38-32 Well & 168 & 2 & 39.9843 & 117.8470 & 1055 & Edwards Creek Valley & Alluvial Fan & Observation well \\
\hline 45-W-5 Well & 59 & 2 & 39.9702 & 117.8582 & 1052 & Edwards Creek Valley & Alluvium & Observation well \\
\hline $45-14$ Well & 102 & 1 & 39.8659 & 118.0049 & 1040 & Carson Sink & Alluvium & Bedrock contact @1830m \\
\hline 46-32 Well (Stu's Well) & 53 & 2 & 39.9881 & 117.8434 & 1061 & Edwards Creek Valley & Alluvial Fan & Quartzite @ $87 \mathrm{~m}$ \\
\hline 62-21 Well & 111 & 1 & 39.9328 & 117.8198 & 1050 & Edwards Creek Valley & Alluvium & Penetrates tuff, gabbro, shale \\
\hline 66-21 Well & 67 & 1 & 39.9311 & 117.9284 & 1040 & Edwards Creek Valley & Alluvium/Alluvial Fan & Bottoms in Humboldt Lopolith \\
\hline 97-2 Well & 122 & 1 & 39.9821 & 117.8406 & 1055 & Edwards Creek Valley & Alluvial Fan & Monitor well; bottoms in alluvium \\
\hline Dixie Jack Gradient Well \#I & near 122 & 1 & 39.9884 & 117.8427 & 1058 & Edwards Creek Valley & Alluvial Fan & In vicinity of $97-2$ well \\
\hline
\end{tabular}


Name or Description

Dixie Jack Gradient Well \#4 Dixie Jack Gradient Well \#7

$$
\text { near }
$$

\section{Background Springs}

Basalt Spring

Big Horn Spring

Dago Spring

Dead Travertine Spring, Upper

Dead Travertine Spring, Road Seep

Dixie Hot Spring

Edward Creek Spring

Fault Line Spring

Horse Creek Spring

Horse Heaven Spring

Hyder Hot Spring

Jersey Hot Spring

Kitten Spring

ш Kyle Spring

Lofthouse Spring

Lower Ranch, Main Hot Spring

Lower Ranch, Upper Warm Spring

McCoy Hot Spring

Mustang Spring

Not-So-OK Spring

Old Man Main Spring

Old Man, Upper Spring

Pine Spring

Sou Hot Springs, Trav. Cone

Sou Hot Springs

Spring in Spring Canyon

Stu's Seep

Upper Cherry Spring

Upper Jersey Seep

War Canyon Spring

Wild Rose Spring

\section{Background Wells}

Bernice Well

Bolivia Artesian Well

Brinkerhoff Well

$\begin{array}{cccccc}\begin{array}{c}\text { Map } \\ \text { Number }\end{array} & \begin{array}{c}\text { Map } \\ \text { Figure }\end{array} & \begin{array}{c}\text { Latitude } \\ (\mathbf{( 2 5} \mathbf{~ m})\end{array} & \begin{array}{c}\text { Longitude } \\ \mathbf{( \pm 2 5 ~ m )}\end{array} & \begin{array}{c}\text { Elevation } \\ \mathbf{( \pm 3} \mathbf{~ m})\end{array} & \begin{array}{c}\text { USGS 1:100,000 } \\ \text { Metric Quadrangle }\end{array} \\ \text { near 122 } & 1 & 39.9852 & 117.8463 & 1057 & \text { Edwards Creek Valley } \\ \text { near 122 } & 1 & 39.9874 & 117.8484 & 1063 & \text { Edwards Creek Valley }\end{array}$

Surface Rocks

Alluvial Fan

Alluvial Fan

Basalt

Alluvium near Gabbro

Alluvium

$39.9081 \quad 117.9865 \quad 1045 \quad$ Edwards Creek Valley

$\begin{array}{llll}40.2137 & 117.8277 & 1505 & \text { Fish Creek Mts }\end{array}$

$\begin{array}{llll}39.9879 & 117.8929 & 1465 \quad \text { Edwards Creek Valley }\end{array}$

$\begin{array}{llll}39.9457 & 117.8972 & 1290 \quad \text { Edwards Creek Valley }\end{array}$

$39.8004 \quad 118.0592 \quad 1040 \quad$ Carson Sink

$39.6819 \quad 117.6444 \quad 1555$

$\begin{array}{lll}40.0317 & 117.6297 \quad 1140\end{array}$

$\begin{array}{lll}39.5238 & 118.0138 & 1560\end{array}$

$39.8348 \quad 117.7220$

$40.0035 \quad 117.7169$

$40.1782 \quad 117.4958$

$40.0550 \quad 117.9159$

$40.1736 \quad 117.7842$

$39.7337 \quad 117.8312$

$40.0335 \quad 117.5981$

$40.0355 \quad 117.6026$

$40.0795 \quad 117.6036$

$40.0879 \quad 117.8774$

$39.7192 \quad 117.8143$

$39.8000 \quad 117.6660$

$39.7962 \quad 117.6822$

$39.6069 \quad 117.8586$

$40.0888 \quad 117.7240$

$40.0890 \quad 117.7240$

$40.1664 \quad 117.6701$

$39.9450 \quad 117.9318$

$\begin{array}{ll}39.5815 & 117.9439\end{array}$

$40.1779 \quad 117.489$

$39.5747 \quad 117.855$

$40.2952 \quad 117.7308$

$39.7706 \quad 117.8092$

$39.9977 \quad 117.9157$

$40.0849 \quad 117.6790$

$$
\text { Carson Sink }
$$

Edwards Creek Valley
Fish Creek Mts

Fish Creek Mts

$$
\text { Carson Sink }
$$

Edwards Creek Valley

Fish Creek Mts

Fish Creek Mts

Fish Creek Mts

Fish Creek Mts

Edwards Creek Valley

Fish Creek Mts

Fish Creek Mts

Fish Creek Mts

Fish Creek Mts

Edwards Creek Valley

Edwards Creek Valley

Edwards Creek Valley

Edwards Creek Valley

Fish Creek Mis

Fish Creek Mts

Fish Creek Mt

Edwards Creek Valley Edwards Creek Valley Fish Creek Mts

Edwards Creek Valley Fish Creek Mts Fish Creek Mts
Alluvium/Alluvial Fan

Fractured Gabbro
Alluvium

Gabbro/Limestone

Alluvium
Gabbro/Quartzite Gabbro/Quartzite

$$
\text { Alluvium }
$$

Alluvium/Alluvial Fan

Welded Tuff

Alluvium/Welded Tuft

Alluvium

Alluvial Fan

Basalt

Quartzite

Alluvium

Alluvial Fan/Limestone Northern-most and hottest spring

Alluvial Fan/Limestone On summit of deposit

Alluvium/Alluvial Fan Near cattle guard

Volcanic sandstone

Quartzite/Argillite

Welded Tuff

Alluvium

Welded Tuff/Basalt

Alluvial Fan/Tuft

Alluvial Fan/Tuff

Lake Beds/Mafic Volc.

Welded Tuff

Travertine/Alluvium

Silicified Welded Tuff

Quartzite/Metavolcanics

South canyon wal

$0.5 \mathrm{~km}$ upstream of main spring

Aka Seven Devils Springs

Hottest spring, constant gas emission
At building foundation

ron hydroxide; flows into creek

Agricultrual well 


\section{Table 1: Continued}

\section{Name or Description}

Hole in the Wall \#2 Well Flowingwell@AA Tank Shaw Well

Unnamed Irrigation Well

\section{Background Streams and Rain}

\section{Bernice Creek}

Bucher Creek

Cedar Canyon Wash

Cottonwood Creek, Lower

Cottonwood Creek, Middle

Dixie Salt Lake

Home Station Wash

Hoyt Creek

Mt. Augusta Creek

Not-So-OK Creek

Rain, Lizard Well Tank

Unnamed Creek by Stu's Seep

Unnamed Stream (east of 121)

White Rock Canyon

\section{Fumaroles}

Calcite Fumarole, Senator area

Crack 4 Fumarole near Stu's Well

Figure 8 Fumarole NE of Senator

Lonely Fumarole SW of Senator

Range Front Fumarole, Senator area

Senator Fumarole

South Bench Fumarole, Senator area

Unnamed Fumarole \#

Unnamed Fumarole \#2

\begin{tabular}{|c|c|c|c|c|c|}
\hline $\begin{array}{l}\text { Map" } \\
\text { Number }\end{array}$ & $\begin{array}{l}\text { Map } \\
\text { Figure }\end{array}$ & $\begin{array}{l}\text { Latitude } \\
( \pm 25 \mathrm{~m})\end{array}$ & $\begin{array}{l}\text { Longitude } \\
( \pm 25 \mathrm{~m})\end{array}$ & $\begin{array}{l}\text { Elevation } \\
( \pm \mathbf{3 ~ m})\end{array}$ & $\begin{array}{c}\text { USGS 1:100,000 } \\
\text { Metric Quadrangle }\end{array}$ \\
\hline 49 & 1 & & & 1345 & Edwards Creek Valley \\
\hline 70 & 1 . & & & 1040 & n Sink \\
\hline 71 & 1 & 39.6812 & 118.0503 & 1048 & Carson Sink \\
\hline 115 & 1 & 40.0986 & 117.6450 & 1125 & Fish Creek Mts \\
\hline
\end{tabular}

$\begin{array}{lll}39.7672 & 117.7897 & 1450 \\ 40.1792 & 117.4913 & 1375 \\ 40.1355 & 117.4791 & 1480 \\ 39.9763 & 117.8808 & 1189 \\ 39.9922 & 117.9128 & 1420 \\ 39.8542 & 118.0000 & 1030 \\ 40.1268 & 117.5117 & 1240 \\ 39.7817 & 117.7897 & 1435 \\ 39.5679 & 117.9229 & 2350 \\ 39.7101 & 117.8119 & 1700 \\ 39.9352 & 117.7189 & 1140 \\ 39.9456 & 117.9317 & 1200 \\ 39.8996 & 117.9953 & 1065 \\ 39.8982 & 118.0180 & 1200\end{array}$

Edwards Creek Valley Fish Creek Mts

Edwards Creek Valley

Edwards Creek Valley

Carson Sink

Fish Creek Mts

Edwards Creek Valley

Edwards Creek Valley

Edwards Creek Valley

Edwards Creek Valley

Edwards Creek Valley

Edwards Creek Valley

Carson Sink

\section{$\begin{array}{llll}39.9924 & 117.8537 & 1152 \quad \text { Edwards Creek Valley }\end{array}$ \\ $\begin{array}{llll}39.9872 & 117.8432 & 1050 \quad \text { Edwards Creek Valley }\end{array}$ \\ $\begin{array}{llll}40.0025 & 117.8424 & 1143 \quad \text { Fish Creek Mts }\end{array}$}

Edwards Creek Valley

Edwards Creek Valley

Edwards Creek Valley

Edwards Creek Valley

Edwards Creek Valley
Fish Creek Mts

$39.9552 \quad 117.9159 \quad 1130 \quad$ Edwards Creek Valley Faulted Alluvial Fan Weak vent; 1 km NE of Stu's Seep

\section{Surface Rocks}

Alluvium near Tuff Alluvium

Alluvium

Alluvium

Quartzite/Argillite Alluvial Fan

Lake Beds/Welded Tuf

Gabbro/Quartzite

Gabbro/Limestone Alluvium

Welded Tuff

Argillite/Shale

Welded Tuff Argillite

Alluvium/Alluvial Fan

Fractured Gabbro

Welded Tuff/Gabbro

Welded Tuff/Gabbro

Below waterfall

Faulted Quartzite Alluvial Fan

Coarse calcite crystals; some sulfur Recent ground crack; some alteration aulted Limestone and Fan Weak vent; minor alteration

Faulted Alluvial Fan Some sulfur

Faulted Gabbro Weak vent: $0.6 \mathrm{~km}$ NE of Stu's Seep

Locations are shown on Figures 1 and 2

"Two samples listed on succeeding tables were mistakenly labeled 181; DV98-181 (Figure 8 Fumarole) and DV99-181 (Goerenger Well). Figure 8 Fumarole uses map number 181. 
Table 2: Field Parameters for Various Geothermal and Regional Waters in the Dixie Valley Region, Nevada.

\begin{tabular}{|c|c|c|c|c|c|c|c|c|c|c|c|}
\hline Sample No. & Name or Description & Date & $\begin{array}{l}\text { Sampling } \\
\text { Temp. }\left({ }^{\circ} \mathrm{C}\right)\end{array}$ & $\begin{array}{l}\text { Sampling } \\
\text { Press. (psig) }\end{array}$ & $\begin{array}{c}\text { Steam } \\
\text { Fraction }(y)\end{array}$ & $\begin{array}{c}\mathrm{pH}^{\mathrm{a}} \\
\text { (field) }\end{array}$ & $\begin{array}{c}\text { Alkalinity } \\
\text { (ppm) }\end{array}$ & $\begin{array}{c}\text { Conduc. } \\
\text { (micromhos) }\end{array}$ & $\begin{array}{c}\text { Eh } \\
(\mathrm{mV})\end{array}$ & $\begin{array}{c}\text { Eh Temp. } \\
\left({ }^{\circ} \mathrm{C}\right)\end{array}$ & Comments \\
\hline \multicolumn{12}{|c|}{ Production Well Brines } \\
\hline DIXE102-W & V102+ V103 Separator & $10 / 02 / 95$ & & ... & 0.153 & --- & -.. & --- & --- & $\ldots$ & Sample provided by L. Shevenell, Univ. Nevada-Reno \\
\hline DV96-8 & $76-7$ Well & $10 / 25 / 96$ & 163 & 110.0 & 0.184 & 9.00 & 137 & 2800 & -75 & 18.7 & Brine flow $=$ about $520,000 \mathrm{lb} / \mathrm{h} ; \mathrm{BHT}=249^{\circ} \mathrm{C}$ \\
\hline DV96-9 & V101 Separator & $10 / 25 / 96$ & 166 & 158.0 & 0.159 & 8.92 & 185 & 2800 & -148 & 31.1 & Brine flow $=1,280,000 \mathrm{lb} / \mathrm{h}$ \\
\hline DV97-II & 73-7 Well & $10 / 29 / 97$ & --- & 85.4 & 0.158 & 8.98 & 178 & 2600 & .298 & 15 & Total flow $=$ about $450,000 \mathrm{lb} / \mathrm{h}$ \\
\hline DV97-13 & 84-7 Well & $10 / 29 / 97$ & --. & 85.5 & 0.159 & 9.01 & 200 & 2600 & -309 & 16.5 & Total tlow = about $200,000 \mathrm{lb} / \mathrm{h} ;$ from $2500 \mathrm{~m}$ \\
\hline DV97-14 & 74-7 Well & $10 / 29 / 97$ & $\ldots$ & 83.8 & 0.163 & 8.84 & 236 & 2700 & -300 & 18.4 & Total flow $=$ about $650,000 \mathrm{lb} / \mathrm{h}$ \\
\hline DV97-16 & V102+V103 Separator & $10 / 29 / 97$ & & 82.2 & 0.161 & 8.77 & 184 & 2800 & -274 & 27.7 & Brine flow $=1,212,000 \mathrm{lb} / \mathrm{h}$ \\
\hline DV97-18 & V105 Separator & $10 / 29 / 97$ &.-- & 108.0 & $0.15 \mathrm{I}$ & 8.68 & 172 & 3300 & -250 & 34 & Brine flow $=1.312,000 \mathrm{lb} / \mathrm{h}$ \\
\hline DV97-20 & 82A-7 Well & $10 / 29 / 97$ & --. & 85.0 & 0.159 & 9.00 & 186 & 2400 & -286 & 17 & Total flow $=$ about $600,000 \mathrm{lb} / \mathrm{h}$ \\
\hline DV97-23 & 73B-7 Well & $10 / 30 / 97$ & & 86.0 & 0.160 & 9.10 & 186 & 2900 & -218 & 20 & \\
\hline DV97-25 & $27-33$ Well & $10 / 30 / 97$ & --- & 97.0 & 0.157 & 8.77 & 222 & 2300 & -282 & 19.8 & Gas in line; poor separation \\
\hline DV97-26 & V101 Separator & $10 / 30 / 97$ & --- & 148.0 & 0.164 & 8.82 & 188 & 2600 & -328 & 17.7 & \\
\hline DV97-29 & 37-33 Well & $10 / 30 / 97$ & -.- & 96.6 & 0.159 & 8.77 & 200 & 2600 & -215 & 34.6 & New well as of July 1997 \\
\hline DV97-30 & 28-33 Well & $10 / 30 / 97$ & $\ldots$ & 101.6 & 0.156 & 8.84 & 182 & 2500 & -272 & 33.4 & \\
\hline DV98-73 & V10I Separator & $04 / 28 / 98$ & 160 & 97.0 & 0.157 & 8.47 & 192 & 2900 & -331 & 56 & \\
\hline DV98-75 & 27-33 Well & $04 / 28 / 98$ & $\ldots$ & 104.0 & 0.155 & 8.49 & 173 & 2600 & -319 & 30 & \\
\hline DV98-77 & 37-33 Well & $04 / 28 / 98$ & 165 & 99.6 & 0.156 & 8.39 & 166 & 2800 & -311 & 48 & \\
\hline DV98-79 & $28-33$ Well & $04 / 28 / 98$ & 168 & 100.0 & 0.157 & 8.82 & 180 & 2300 & -313 & 24 & \\
\hline DV98-80 & 76A-7 Well & $04 / 28 / 98$ & --- & 88.5 & 0.157 & 8.66 & 160 & 2700 & -308 & 31.2 & \\
\hline DV98-82 & V102+V103 Separator & $04 / 28 / 98$ & --- & 87.3 & 0.150 & 8.53 & 146 & 2900 & -310 & 30 & \\
\hline DV98-84 & 74-7 Well & $04 / 28 / 98$ & --- & 89.8 & 0.158 & 8.63 & 160 & 2400 & -311 & 26.2 & \\
\hline DV98-86 & 63-7 Well & $04 / 28 / 98$ & --- & 88.8 & 0.154 & 8.62 & 150 & 2500 & -280 & 23.9 & \\
\hline DV98-88 & 73-7 Well & $04 / 29 / 98$ & --- & 90.5 & 0.154 & 8.63 & 158 & 2900 & -303 & 25 & \\
\hline DV98-90 & 82A-7 Well & $04 / 29 / 98$ & --- & 90.6 & 0.153 & 8.85 & 170 & 2600 & -285 & 24.2 & \\
\hline DV98-92 & V105 Separator & $04 / 29 / 98$ & --- & 86.5 & 0.150 & 8.74 & 165 & 2800 & -295 & 36 & \\
\hline DV98-95 & 73B-7 Well & $04 / 29 / 98$ & 174 & 92.5 & 0.152 & 8.73 & 156 & 2300 & -296 & 31.1 & \\
\hline DV98-133 & $27-33$ Well & $10 / 20 / 98$ & --- & 94.0 & --- & 8.57 & --- & $\ldots$ & & & Backflow from separator: $\mathrm{BHT}=243^{\circ} \mathrm{C}$ \\
\hline DV98-135 & $27-33$ Well & $10 / 20 / 98$ & --- & 52.0 & 0.184 & 8.77 & --- & --- & --- & --- & Off line \\
\hline DV98-138 & V101 Separator & $10 / 21 / 98$ & --- & 151.2 & 0.160 & 8.78 & --- & --- & -.- & -.. & \\
\hline DV98-140 & $37-33$ Well & $10 / 21 / 98$ & -.- & 90.0 & 0.162 & 8.82 & 194 & $\ldots$ & & & \\
\hline DV98-141 & $28-33$ Well & $10 / 21 / 98$ & -.- & 108.0 & 0.162 & 8.84 & 190 & --- & $\cdots$ & $\ldots$ & $\mathrm{BHT}=246^{\circ} \mathrm{C}$ \\
\hline DV98-145 & $76 \mathrm{~A}-7$ Well & $10 / 22 / 98$ & --. & 112.0 & 0.158 & 8.50 & 192 & $\ldots$ & -.- & --- & \\
\hline DV98-147 & 63-7 Well & $10 / 22 / 98$ & & 87.2 & 0.155 & 8.60 & 192 & -.- & --- & --- & $\mathrm{BHT}=241^{\circ} \mathrm{C}$ \\
\hline DV98-148 & V102+V103 Separator & $10 / 22 / 98$ & 166 & --- & 0.164 & 8.58 & 174 & --- & --- & --- & \\
\hline DV98-150 & 74-7 Well & $10 / 22 / 98$ & --. & --- & 0.160 & 8.71 & 170 & --- & --- & --- & $\mathrm{BHT}=244^{\circ} \mathrm{C}$ \\
\hline DV98-152 & 73-7 Well & $10 / 22 / 98$ & --- & 93.0 & 0.154 & 8.53 & 168 & --- & --- & --- & \\
\hline DV98-154 & 73B-7 Well & $10 / 22 / 98$ & --- & 96.0 & 0.154 & 8.89 & 160 & -.- & ... & $\ldots$ & \\
\hline DV98-156 & $82 \mathrm{~A}-7$ Well & $10 / 23 / 98$ & & --- & 0.154 & 8.85 & 190 & -.- & --- & --- & $\mathrm{BHT}=241^{\circ} \mathrm{C}$ \\
\hline DV98-159 & V105 Separator & $10 / 23 / 98$ & $143 \%$ & 110.5 & 0.146 & 8.79 & 198 & --- & ... & & \\
\hline DV99-182 & 76A-7 Well & $05 / 04 / 99$ & --- & $92.0 \mathrm{psi} W \mathrm{H}$ & 0.152 & 9.27 & 155 & --- & -70 & 21.7 & \\
\hline DV99-184 & $74-7 \mathrm{Well}$ & $05 / 04 / 99$ & --- & 85.5 & 0.160 & 9.55 & 162 & --- & -170 & 19.4 & \\
\hline DV99-186 & V102 + V103 Separator & $05 / 04 / 99$ & --- & $83.5 \mathrm{psi}$ & 0.137 & 9.12 & 159 & --- & -228 & 56.6 & \\
\hline DV99- 188 & 63-7 Well & $05 / 04 / 99$ & --- & 90.0 & 0.152 & 9.44 & 168 & $\ldots$ & -178 & 18.9 & \\
\hline DV99-190 & 73-7 Well & $05 / 04 / 99$ & $\ldots--$ & 88.0 & 0.154 & 9.21 & 141 & $\ldots$ & -172 & 39.4 & \\
\hline DV99-194 & V105 Separator & $05 / 05 / 99$ & $\ldots$ & $83.8 \mathrm{psi}$ & 0.138 & 8.74 & 138 & $\ldots$ & $387 ?$ & 62.4 & \\
\hline DV99-196 & 82A-7 Well & $05 / 05 / 99$ & -.- & 87.8 & 0.152 & 8.86 & 148 & --- & -151 & 56.3 & \\
\hline
\end{tabular}


Table 2: Continued

\begin{tabular}{|c|c|c|c|c|c|c|c|c|c|c|c|}
\hline Sample No. & Name or Description & Date & $\begin{array}{l}\text { Sampling } \\
\text { Temp. }\left({ }^{\circ} \mathrm{C}\right)\end{array}$ & $\begin{array}{l}\text { Sampling } \\
\text { Press. (psig) }\end{array}$ & $\begin{array}{c}\text { Steam } \\
\text { Fraction }(y)\end{array}$ & $\begin{array}{l}\mathrm{pH}^{*} \\
\text { (field) }\end{array}$ & $\begin{array}{c}\text { Alkalinity } \\
\text { (ppm) }\end{array}$ & $\begin{array}{c}\text { Conduc. } \\
\text { (micromhos) }\end{array}$ & $\begin{array}{c}\mathbf{E h} \\
(\mathbf{m V})\end{array}$ & $\begin{array}{c}\text { Eh T Tump. } \\
\left({ }^{\circ} \mathrm{C}\right)\end{array}$ & Comments \\
\hline DV99-197 & 73B-7 Well & $05 / 05 / 99$ & -- & 80.5 & 0.159 & 9.48 & 150 & --- & -190 & 30.7 & \\
\hline DV99-199 & 37-33 Well & $05 / 05 / 99$ & -- & 93.7 & 0.160 & 9.21 & 207 & --- & -437 & 43.1 & \\
\hline DV99-200 & 28-33 Well & 05/05/99 & --- & 97.0 & 0.159 & 8.98 & 193 & --- & -413 & 59.7 & \\
\hline DV99-204 & V101 Separator & 05/05/99 & --- & 92.5 & 0.159 & 9.04 & 198 & -- & -422 & 42.6 & \\
\hline DV74782786-brine 2 & 74-7 Well Archived & $08 / 27 / 86$ & $\ldots$ & --- & 0.199 & --- & -. & --- & --- & --- & Archived brine sample analyzed by LANL \\
\hline DV76781986-brine 4 & 76-7 Well Archived & $08 / 19 / 86$ & --- & -- & 0.187 & $\cdots$ & $\ldots$ & --- & -- & --- & Archived brine sample analyzed by LANL \\
\hline DV453382186-brine 6 & 45-33 Well Archived & $08 / 21 / 86$ & --- & --- & 0.165 & -- & --- & --- & -.- & --- & Archived brine sample analyzed by LANL \\
\hline DV73782886-brine 8 & 73-7 Well Archived & $08 / 28 / 86$ & $\cdots$ & $\cdots$ & 0.198 & --- & -- & -- & -- & --- & Archived brinc sample analyzed by LANL \\
\hline DV321882686-brine 10 & 32-18 Well Archived & $08 / 26 / 86$ & --- & $\cdots$ & -- & --- & --- & $\cdots$ & -- & -.- & Archived brine sample analyzed by LANL \\
\hline DV651882686-brine 12 & 65-18 Well Archived & $08 / 26 / 86$ & --- & $\cdots$ & -- & --- & --- & -. & --- & --- & Archived brine sample analyzed by LANL \\
\hline No number & 28-33 Well Archived & 09/23/93 & $\cdots$ & $\cdots$ & 0.158 & $\cdots$ & $\cdots$ & --- & $\cdots$ & $\cdots$ & Warm aquifer $\sim 1,200^{\prime}$ from liner hanger \\
\hline \multicolumn{12}{|c|}{ Production Well Condensates } \\
\hline DIXE102-S & V102+V103 Separator & $10 / 02 / 95$ & & $\ldots$ & --- & --- & ..- & -.. & --- & --- & Sample provided by L. Shevenell, Univ. Nevada-Reno \\
\hline DV96-7 & 76-7 Well & $10 / 25 / 96$ & 163 & 110 & --- & 6.68 & 45 & --- & -17.8 & 61.6 & Steam flow $=115,000 \mathrm{lb} / \mathrm{h}$ \\
\hline DV96-10 & V101 Separator & $10 / 25 / 96$ & 166 & 158 & --- & --. & --- & --- & -- & --- & Steam flow $=250,000 \mathrm{lb} / \mathrm{h}$ \\
\hline DV97-12 & 73-7 Well & $10 / 29 / 97$ & --- & 85.4 & -- & -- & -- & --- & --- & -.. & Brine carry over in sample \\
\hline DV97-15 & 74.7 Well & $10 / 29 / 97$ & -- & 83.8 & -.. & $\cdots$ & -- & $\cdots$ & --- & --- & \\
\hline DV97-17 & V102+V103 Separator & $10 / 29 / 97$ & --- & 80.2 & --- & --- & -- & -.- & $\cdots$ & -- & Steam flow $=230,000 \mathrm{lb} / \mathrm{h}$ \\
\hline DV97-19 & V105 Separator & $10 / 29 / 97$ & ... & 81.3 & -- & -- & -- & -.. & & $\cdots$ & Steam flow $=227,000 \mathrm{lb} / \mathrm{h}$ \\
\hline DV97-2I & $82 \mathrm{~A}-7 \mathrm{Well}$ & $10 / 29 / 97$ & $\ldots$ & 84.7 & --- & -- & -.- & -- & -- & --. & \\
\hline DV97-22 & 73B-7 Well & $10 / 29 / 97$ & $\cdots$ & 84 & $\cdots$ & $\cdots$ & --- & -- & $\cdots$ & -- & \\
\hline DV97-24 & V101 Separator & $10 / 30 / 97$ & --- & 90.6 & --- & -.- & -- & -- & --- & --- & \\
\hline DV97-27 & $27-33$ Well & $10 / 30 / 97$ & $\cdots$ & 97 & $\cdots$ & $\cdots$ & --- & $\cdots$ & --- & $\cdots$ & \\
\hline DV97-28 & 37-33 Well & $10 / 30 / 97$ & --- & 96.6 & $\ldots$ & --- & $\ldots$ & -- & --- & --- & New well as of July 1997 \\
\hline DV97-31 & 28-33 Well & $10 / 30 / 97$ & 167.2 & --- & --- & -- & & -- & -- & --- & From mini-sep@wellhead \\
\hline DV98-74 & V101 Separator & $04 / 28 / 98$ & 160 & 104 & -- & --- & -.- & -- & -- & -- & \\
\hline DV98-76 & 27-33 Well & $04 / 28 / 98$ & --- & 104 & --- & --- & --- & $\ldots$ & $\ldots$ & --- & \\
\hline DV98-78 & 37-33 Well & $04 / 28 / 98$ & $\ldots$ & 99.5 & --- & --- & -- & $\cdots$ & $\cdots$ & --- & \\
\hline DV98-81 & $76 \mathrm{~A}-7 \mathrm{Well}$ & $04 / 28 / 98$ & --- & 88.5 & $\ldots$ & --- & --- & -.- & --- & --- & \\
\hline DV98-83 & V102 + V103 Separator & $04 / 28 / 98$ & --- & 87.25 & -- & $\cdots$ & -- & --- & --- & $\cdots$ & \\
\hline DV98-85 & 74-7 Well & $04 / 28 / 98$ & --- & 88.7 & & & -.- & & --- & -- & \\
\hline DV98-87 & 63-7 Well & $04 / 28 / 98$ & $\cdots$ & 88.8 & $\cdots$ & --- & $\cdots$ & -- & --- & -- & \\
\hline DV98-89 & 73-7 Well & $04 / 29 / 98$ & -- & 90.5 & -- & $\cdots$ & -.- & -- & --- & -- & Brine carry over in sample \\
\hline DV98-91 & 82A-7 Well & $04 / 29 / 98$ & --- & 89.8 & --- & $\cdots$ & & -- & $\ldots$ & --- & Brine carry over in sample \\
\hline DV98-93 & V105 Separator & $04 / 29 / 98$ & --- & 86.5 & -- & -- & $\cdots$ & --- & $\cdots$ & --- & \\
\hline DV98-94 & 73B-7 Well & $04 / 29 / 98$ & 174 & 90.5 & --- & --- & --- & -- & --- & --- & \\
\hline DV98-10! & 28-33 Well & $04 / 30 / 98$ & 168 & 99.5 & -- & -- & -- & & $\ldots$ & --- & \\
\hline DV98-136 & 27-33 Well & $10 / 20 / 98$ & -.- & 94 & -.. & --- & --- & $\cdots$ & $\cdots$ & $\cdots$ & Off line \\
\hline DV98- 137 & V lo! Separator & $10 / 21 / 98$ & --- & 83 & $\cdots$ & --- & --- & $\cdots$ & $\cdots$ & -- & \\
\hline DV98-139 & 37-33 Well & $10 / 21 / 98$ & --- & 89.1 & -.. & --- & -- & -- & -- & $\cdots$ & \\
\hline DV98-142 & 28-33 Well & $10 / 21 / 98$ & --- & 89.2 & -- & $\cdots$ & $\cdots$ & --- & --- & --- & \\
\hline DV98-144 & 76A-7 Well & $10 / 22 / 98$ & --- & 83.3 & & $\ldots$ & $\cdots$ & $\cdots$ & --- & --- & \\
\hline DV98-146 & V102+V103 Separator & $10 / 22 / 98$ & --- & 82.7 & ..- & -- & $\ldots$ & -.. & $\ldots$ & --- & \\
\hline DV98-149 & $63-7$ Well & $10 / 22 / 98$ & --- & 87.2 & $\cdots$ & -- & $\cdots$ & $\cdots$ & -- & --- & $\mathrm{BHT}=241^{\circ} \mathrm{C}$ \\
\hline DV98-151 & 74-7 Well & $10 / 22 / 98$ & -.- & 85.4 & --- & -.- & -. & -- & $\cdots$ & $\cdots$ & \\
\hline DV98-153 & 73-7 Well & $10 / 22 / 98$ & -- & 87.5 & $\cdots$ & $\cdots$ & $\cdots$ & $\cdots$ & -- & -- & Brine carry over in sample \\
\hline
\end{tabular}




\section{Table 2: Continued}

\begin{tabular}{|c|c|c|c|c|c|c|c|c|c|c|c|}
\hline Sample No. & Name or Description & Date & $\begin{array}{l}\text { Sampling } \\
\text { Temp. }\left({ }^{(} \mathbf{C}\right)\end{array}$ & $\begin{array}{l}\text { Sampling } \\
\text { Press. (psig) }\end{array}$ & $\begin{array}{c}\text { Steam } \\
\text { Fraction }(y)\end{array}$ & $\begin{array}{c}\mathbf{p H}^{*} \\
\text { (field) }\end{array}$ & $\begin{array}{l}\text { Alkalinity } \\
\text { (ppm) }\end{array}$ & $\begin{array}{c}\text { Conduc. } \\
\text { (micromhos) }\end{array}$ & $\begin{array}{l}\text { Eh } \\
(\mathbf{m V})\end{array}$ & $\begin{array}{c}\text { Eh Temp. } \\
\left.\text { ( }{ }^{\circ} \mathrm{C}\right)\end{array}$ & Comments \\
\hline DV98-155 & 73B-7 Well & $10 / 22 / 98$ & --- & 87.4 & --- & --- & --- & --- & -.. & --- & Brine carry over in sample \\
\hline DV98-157 & 82A-7 Well & $10 / 23 / 98$ & $\cdots$ & 86.5 & --- &.-- & $\cdots$ & --- & --- & --- & Brine carry over in sample \\
\hline DV98-158 & V105 Separator & $10 / 23 / 98$ & 163 ? & 84.5 & --- & --- & & $\cdots$ & --- & --- & \\
\hline DV99-183 & 76A-7 Well & 05/04/99 & --- & --- & $\cdots$ & --- & --- & -.- & --- & --- & \\
\hline DV99-185 & 74-7 Well & $05 / 04 / 99$ & $\cdots$ & --- & $\cdots$ & $\ldots$ & --- & --- & $\cdots$ & $\cdots$ & Brine carry over in sample \\
\hline DV99-187 & V102+V103 Separator & 05/04/99 & --- & --- & --- & & $\cdots$ & --- & --- & --- & \\
\hline DV99-189 & 63-7 Well & 05/04/99 & $\cdots$ & 87.3 & --- & --. & --- & --- & -.. & --- & \\
\hline DV99-191 & 73-7 Well & $05 / 04 / 99$ & & 88 & --- & --- & --- & --. & & & Brine carry over in sample \\
\hline DV99-192 & 73B-7 Well & $05 / 04 / 99$ & & 87 & -.- & --- & --- & --- & ... & -.. & Brine carry over in sample \\
\hline DV99-193 & V105 Separator & 05/05/99 & --- & --- & -.- & --- & --- & ... & $\ldots$ & $\ldots$ & \\
\hline DV99-195 & 82A-7 Well & 05/05/99 & --- & 86.5 & -.- & --- & $\ldots$ & & -.- & --- & Brine carry over in sample \\
\hline DV99-201 & 28-33 Well & 05/05/99 & $\ldots$ & 96.7 & --- & --- & -.. & $\cdots$ & --- & --- & \\
\hline DV99-202 & 37-33 Well & 05/05/99 & -.- & 96 & --- & --- & -.. & --- & -.- & --- & \\
\hline DV99-203 & V101 Separator & 05/05/99 & $\cdots$ & 90 & --- & --- & $\cdots$ & & --- & --- & \\
\hline DV74782786-cond I & 74-7 Well Archived & $08 / 27 / 86$ & --- & & --- & --- & --- & --- & --. & --- & Archived condensale sample analyzed by LANL \\
\hline DV76781986-cond 3 & 76-7 Well Archived & $08 / 19 / 86$ & --- & -.. & --- & --- & $\cdots$ & $\cdots$ & --- & --- & Archived condensate sample analyzed by LANL \\
\hline DV453382886-cond 5 & 45-33 Well Archived & $08 / 28 / 86$ & --- & -.. & --- & --- & $\ldots$ & & --- & --- & Archived condensate sample analyzed by LANL \\
\hline DV73782886-cond 7 & 73-7 Well Archived & $08 / 28 / 86$ & --- & & --- & --- & -.- & --- & --- & --- & Archived condensate sample analyzed by LANL \\
\hline DV $321882686-$ cond 9 & 32-18 Well Archived & $08 / 26 / 86$ & --- & --- & --- & --. & --- & -.- & -.. & -.. & Archived condensate sample analyzed by LANL \\
\hline DV651882686-cond 11 & 65-18 Well Archived & $08 / 26 / 86$ & --- & --- & --- & --- & $\cdots$ & & $\cdots$ & -- & Archived condensate sample analyzed by LANL \\
\hline \multicolumn{12}{|c|}{ Injection Well/Power Plant Fluids } \\
\hline DV96-2 & Condensate from plant & $10 / 24 / 96$ & 41.8 & $\ldots$ & --- & 6.28 & 7 & 300 & 363 & 37.9 & Mix of condensate, raw steam, minor $\mathrm{NaOH}$ \\
\hline DV96-3 & LP brine @ plant & $10 / 24 / 96$ & 110 & & --- & 9.31 & 184 & 2700 & -135 & 29.3 & Low-pressure spent brine \\
\hline DV96-4 & 45-5 Injection Well & $10 / 24 / 96$ & 107 & 10.5 & --- & 8.77 & 180 & --- & -145 & 66 & Spent brine + condensed steam; rate $=4.500 \mathrm{gpm}$ \\
\hline DV96-5 & Lamb I Injection Well & $10 / 24 / 96$ & 107 & 14 & --- & 8.99 & 184 & --- & -120 & 46.5 & Pure spent brine: rate $=475 \mathrm{gpm}$ \\
\hline DV96-6 & 65-18 Injection Well & $10 / 24 / 96$ & $>60$ & -- & --- & 8.67 & 198 & 2800 & -145 & 55 & Pure spent brine \\
\hline DV97-32 & Condensate from plant & $10 / 31 / 97$ & 40 & $\cdots$ & --- & --- & $\ldots$ & -- & $\cdots$ & $\cdots$ & From reinjection line \\
\hline DV97-33 & LP brine@plant & $10 / 31 / 97$ & 107.2 & 38 & --- & 9 & 204 & 3100 & -267 & 35 & From reinjection line \\
\hline DV97-34 & $25-5+45-5$ injectate & $10 / 31 / 97$ & 104.4 & 80 & --- & 9.14 & 186 & 2700 & -160 & 25.7 & Brine + cond, well mixed \\
\hline DV97-35 & $25-5+45-5$ injectate & $10 / 31 / 97$ & 99.7 & 9 & --- & 8.93 & 164 & 3100 & -246 & 43.9 & Line by $N$ injection wells $25-5 \& 45-5$ \\
\hline DV97-36 & 65-18 Injection Well & $10 / 31 / 97$ & 108 & 27 & -.- & 8.95 & 196 & 3200 & -185 & 45 & Pure spent brine \\
\hline DV97-37 & 32-18 Injection Well & $10 / 31 / 97$ & 102 & & & 9.07 & 210 & 3000 & -225 & 35.8 & \\
\hline DV97-40 & LP brine@plant & $10 / 31 / 97$ & $\ldots$ & 100 & -.- & 8.97 & 178 & 3100 & -245 & 40.1 & \\
\hline DV97-41 & Condensate from plant & $10 / 31 / 97$ & 27.8 & --- & --- & --- & -- & --- & --- & -- & Al cooling tower \\
\hline DV97-42 & High press. brine @ plant & $10 / 31 / 97$ & --- & 89.4 & & 8.86 & 195 & 2300 & -172 & 25.3 & North line above LP separators \\
\hline DV98-97 & Condensate from plant & $04 / 29 / 98$ & 41.0 & $\cdots$ & --- & 6.29 & -- & -- & $\ldots$ & --- & From "hot well" line: sent to $65-18$ injector \\
\hline DV98-98 & LP brine @ plant & $04 / 29 / 98$ & 110 & 94 & -.- & 9.07 & 166 & 2400 & -307 & 35.7 & Flow rate $=9.000 \mathrm{gpm}$ \\
\hline DV98-143 & 25-5 Injection Well & $10 / 21 / 98$ & -.- & 13.2 & --- & $8.88(6.1)$ & 190 & --- & --- & $\ldots$ & LP brine \\
\hline DV98-161 & Condensate from plant & $10 / 23 / 98$ & 40.4 & --- & --- & 5.5 & --- & --- & --- & --- & From "hot well" line \\
\hline DV98- 162 & LP Brine @ Plant & $10 / 23 / 98$ & 100 & 100 & --- & $9.29(27.2)$ & 216 & & & --- & \\
\hline DV98-163 & 65-18 Injection Well & $10 / 23 / 98$ & $>30$ & & --- & 7.20 & 182 & --- & -- & -- & Injection Manifold Total fluid \\
\hline DV99-198 & 65-18 Injection Well & $05 / 05 / 99$ & $\cdots$ & -- & -.- & 7.4 & 174 & --- & 181 & 31.4 & \\
\hline DV99-205 & 25-5 + 45-5 Injectate & $05 / 06 / 99$ & $\cdots$ & $12 \mathrm{psi} \mathrm{WH}$ & --- & $9.31(25.2)$ & 214 & & 341 & 39.9 & \\
\hline DV99-206 & L.PBrine @Plant & $05 / 06 / 99$ &.-- & --- & --- & $9.32(27.9)$ & 170 & $\cdots$ & -89 & 48 & \\
\hline DV99-207 & Condensate from plant & $05 / 06 / 99$ & $\cdots$ & $\cdots$ & $\cdots$ & 6.67 & 37 & -- & 52 & 38.6 & From "hot well" line \\
\hline DV99-208 & $52-18+41-18$ lnjectate & $05 / 06 / 99$ & --- & --- & $\cdots$ & $9.08(15.0)$ & 166 & -.- & -108 & 51.4 & \\
\hline
\end{tabular}


Table 2: Continued

\section{Sample No. \\ Name or Description}

Other Geothermal and On-Site Water Wells

DV96-1

DV97-38

DV97-39

DV97-53
DV97-54

DV97-54

DV97-59

DV97-67

DV98-96

DV98-99

DV $98-99$

DV98-102

DV98-103

DV98-104

DV98-111

DV98- 122

DV98-123

Dixie Jack \#1

Dixie Jack \#4

Dixie Jack \#

$\bar{\infty} \quad$ DV $98-160$

DV98-168

DV99-181

Background Springs

DV97-46

DV $97-47$

DV97-48

DV97-50

DV $97-51 \mathrm{a}$

DV $97-5 \mathrm{lb}$

DV97-52

DV97-56

DV97-60

DV97-61

DV97-62

DV97-63

DV97-64

DV97-65

DV97-66

DV97-68

DV97-69

DV97-72

DV98-106

DV98-112
Domestic Well

Domestic Well

Goerenger Well

46-32 Well

27-32 Well

27-32 Well
45-W- 5 Well

66-21 Well

Goerenger Well

27-32 Well

46-32 Well

45-14 Well

45-14 Wcll

66-2I Well

62-21 Well

97-2 Well

Gradient Well DJ\#1

Gradient Well DJ \#4

Gradient Well DJ \#?

Goerenger Well

38-32 Well

Goerenger Wel

Sou Hot Spring

Sou Hot Spring

Hyder Hot Spring

Edward Crcek Spring

Old Man Spring. Uppe

Old Man Main Spring

Horse Heaven Spring

Dead Travertine Spring

Fault Line Spring

Lower Ranch Hot Spring

McCoy Hot Spring

Kyle Spring

Dago Spring

Mustang Spring

Kitten Spring

Big Horn Spring

Dixie Hot Spring

Horse Creek Spring

Stu's Seep

Hyder Hot Spring
Date

$\begin{array}{cccc}\text { Sampling } & \text { Sampling } & \text { Steam } & \mathrm{pH}^{a} \\ \text { Temp. }\left({ }^{\circ} \mathrm{C}\right) & \text { Press. (psig) } & \text { Fraction }(\mathrm{y}) & \text { (field) }\end{array}$

Alkalinity Conduc. Eh Eh Tamp.

(ppm) (micromhos) $\quad(\mathrm{mV}) \quad\left({ }^{\circ} \mathrm{C}\right)$

$\begin{array}{lllllllll}10 / 31 / 97 & 29.2 & -. & -- & 7.46 & 244 & 1100 & 91.5 & 26.9 \\ 10 / 3197 & 27.8 & - & - & 7.13 & 312 & 1600 & 91.7 & 27.8\end{array}$

$\begin{array}{lllllllll}10 / 31 / 97 & 27.8 & - & -- & 7.13 & 312 & 1600 & 91.7 & 27.8 \\ 11 / 05 / 97 & 155 & 23 & -3 & & & & & \end{array}$

1105197

$11 / 05 / 97$

$11 / 05 / 97$

$11 / 07 / 97$

$04 / 29 / 98$

$04 / 29 / 98$

$04 / 29 / 98$

$04 / 30 / 98$

$04 / 30 / 98$

$04 / 30 / 98$

$05 / 01 / 98$

$05 / 05 / 98$

$05 / 17 / 98$

$05 / 20198$

$05 / 20 / 98$

$05 / 14 / 98$

$10 / 23 / 98$

$10 / 26 / 98$

$10 / 28 / 98$

$05 / 04 / 99$

$--$

144

166
26.4

55.5

28.3

$--$

Depth $=73 \mathrm{~m}$; cased to $67 \mathrm{~m}$

Depth $=101 \mathrm{~m}$; static press. $=45 \mathrm{psig}$

Depth $=296 \mathrm{~m}$; static press. $=80 \mathrm{psi}$

Producing fractures (a) $148 \mathrm{~m}$ depth

Depth $=6.9 \mathrm{~m}$

-.- Artesian flow $=7 \mathrm{l} / \mathrm{min}$; depth $=2,988 \mathrm{~m}$

1500

$\cdots$

Flow $=965 \mathrm{gpm}$, pumped; depth $=73 \mathrm{~m}$

Static press. $=62 \mathrm{psig}$

Static press. $=13 \mathrm{psig}$

Depth $=2440 \mathrm{~m} ;$ condensate sampled $\mathrm{w} /$ minisep.

Brine sampled $w /$ minisep.

Sporadic artesian flow $=41 / \mathrm{min}$

40 Depth $=3810 \mathrm{~m} ; \mathrm{BHT}=190^{\circ} \mathrm{C} ;$ flow $=36 \mathrm{gpm}$

Depth $=61 \mathrm{~m}$; no flow

Depth $=152 \mathrm{~m}$; flow 0 to $150 \mathrm{gpm}$

From $76 \mathrm{~m}$ depth during drilling and circulation

Artesian flow $=7 \mathrm{gpm} @ 67 \mathrm{~m}$ depth

From $53 \mathrm{~m}$ depth during drilling; steam @ $61 \mathrm{~m}$

Flow $=620$ gpm pumped

- Depth to water $=9.1 \mathrm{~m}$

..- Production @ 2,440 to 2,900 m; from gabbro

$68 \mathrm{psi}$

$11 / 03 / 97$

$11 / 03 / 97$

$11 / 03 / 97$

$11 / 04 / 97$

$11 / 04 / 97$

$11 / 04 / 97$

$11 / 04 / 97$

$11 / 05 / 97$

$11 / 06 / 97$

$11 / 06 / 97$

$11 / 06 / 97$

$11 / 06 / 97$

$11 / 06 / 97$

$11 / 06 / 97$

$11 / 06 / 97$

$11 / 07 / 97$

$11 / 07 / 97$

$11 / 07 / 97$

$04 / 30 / 98$

$04 / 30 / 98$

57.0
72.
76
13.
10.8
13.2
17.4
28.8
40.8
46
19.8
13.
14
16.
20.5
81.
14.
17.2
75

Pool in large travertine cone; no observed flow

Hottest spring with gas: flow $=4 \mathrm{l} / \mathrm{min}$

- Summit of deposit: flow $=8 \mathrm{l} / \mathrm{min}(120 \mathrm{l} / \mathrm{min}$ total

From broken corral: flow $=1 \mathrm{l} / \mathrm{min}$

-. Isotope sample only; seep

--- $\quad$ Flow $=0.5 \mathrm{l} / \mathrm{min}$

Flow $=0.5 \mathrm{l} / \mathrm{min}$

- Seep

Flow = at least $21 / \mathrm{min}$

Northern-most hottest spring; flow $\geq 1201 / \mathrm{min}$

Flow $\geq 50 \mathrm{l} / \mathrm{min}$

-. Flow $=30 \mathrm{l} / \mathrm{min}$

Flow $=1 \mathrm{l} / \mathrm{min}$

Flow $=251 / \mathrm{min}$

Flow $=51 / \mathrm{min}$

Flow $=1 \mathrm{l} / \mathrm{min}$

Hotest spring near road; flow $=101 / \mathrm{min}$

Flow $=1 \mathrm{l} / \mathrm{min}$

Seep 
Table 2: Continued

\begin{tabular}{|c|c|c|c|c|c|c|c|c|c|c|c|}
\hline Sample No. & Name or Description & Date & $\begin{array}{l}\text { Sampling } \\
\text { Temp. }\left({ }^{\circ} \mathrm{C}\right)\end{array}$ & $\begin{array}{l}\text { Sampling } \\
\text { Press. (psig) }\end{array}$ & $\begin{array}{l}\text { Steam } \\
\text { Fraction }(y)\end{array}$ & $\begin{array}{c}\mathbf{p H}^{*} \\
\text { (field) }\end{array}$ & $\begin{array}{l}\text { Alkalinity } \\
\text { (ppm) }\end{array}$ & $\begin{array}{c}\text { Conduc. } \\
\text { (micromhos) }\end{array}$ & $\begin{array}{c}\text { Eh } \\
(\mathrm{mV})\end{array}$ & $\begin{array}{l}\text { Eh Temp. } \\
\quad\left({ }^{\circ} \mathrm{C}\right)\end{array}$ & Comments \\
\hline DV98-113 & Lower Ranch Hot Spring & $05 / 04 / 98$ & 40.4 & --- & -.- & 7.05 & --- & --- & --. & -.- & Northern-most hotest spring; flow $=1001 / \mathrm{min}$ \\
\hline DV98-114 & McCoy Hot Spring & $05 / 04 / 98$ & 46.0 & & --- & 7.05 & --- & -.. & --- & $\cdots$ & Flow not measured \\
\hline DV98-117 & Sou Hot Spring & $05 / 04 / 98$ & 72.0 & --- & $\cdots$ & 6.46 & --- & $\cdots$ & --- & --- & Hottest spring with gas: flow $=41 / \mathrm{min}$ \\
\hline DV98-118 & Big Horn Spring & 05/04/98 & 18.1 & --- & $\cdots$ & 7.35 & $\cdots$ & $\cdots$ & $\cdots$ & --- & Seep \\
\hline DV98-120 & Dixie Hot Spring & $05 / 05 / 98$ & 83.5 & --- & & 8.14 & --- & & $\cdots$ & $\cdots$ & Hottcst spring near road; $110 \mathrm{w}=10 \mathrm{l} / \mathrm{min}$ \\
\hline DV98-128 & Jersey Hot Spring & $05 / 05 / 98$ & 59.0 & --- & $-\cdots$ & 7.0 & $\cdots$ & --- & --- & --- & Flow $=200 \mathrm{l} / \mathrm{min}$ \\
\hline DV98-129 & Upper Jersey Seep & $05 / 06 / 98$ & 17.5 & --- & --- & 7.0 & -- & --- & --- & --- & Seep \\
\hline DV98-131 & Spring in Spring Canyon & $05 / 06 / 98$ & 14.0 & $\cdots$ & --- & 7.0 & -- & --- & & --- & $\mathrm{Flow}=1 \mathrm{l} / \mathrm{min}$ \\
\hline DV98-1.32 & Wild Rose Spring & $05 / 07 / 98$ & 8.0 & --- & --- & 6.5 & --- & --- & --- & --- & Flow $=201 / \mathrm{min}$ \\
\hline DV98-169 & Lofthouse Spring & $05 / 07 / 98$ & 14.8 & $-\cdots$ & --- & 6.5 & --- & --- & --- & --- & Flow $=81 / \mathrm{min}$ \\
\hline DV98-170 & Not-So-OK Spring & $10 / 27 / 98$ & 10.0 & $-\cdots$ & $\cdots$ & 6.5 & $\cdots$ & --- & & $\cdots$ & Flow $=41 / \mathrm{min}$ \\
\hline DV98-176 & War Canyon Spring & $10 / 27 / 98$ & 11.2 & --- & --- & 6.5 & --- & --- & --- & --- & Flow $=1 \mathrm{l} / \mathrm{min}$ \\
\hline DV98-177 & Pine Spring & $10 / 28 / 98$ & 8.6 & & --- & 6.8 & $\cdots$ & -- & $\cdots$ & --- & Flow $=20 \mathrm{l} / \mathrm{min}$ \\
\hline DV98-178 & Basalt Spring & $10 / 28 / 98$ & 8.4 & --- & $\cdots$ & 6.5 & --- & --- & $\cdots$ & --- & Flow $=2 \mathrm{l} / \mathrm{min}$ \\
\hline DV98-179 & Upper Cherry Spring & $10 / 28 / 98$ & 7.4 & --- & --- & 6.5 & --- & --- & --- & & Flow $=41 / \min$ \\
\hline DV99-209 & Dead Travertine Spring & $05 / 07 / 99$ & 19.2 & & --- & 6.7 & $\cdots$ & --- & --- & $\cdots$ & Seep \\
\hline DV99-210 & Dead Travertine, road scep & $05 / 08 / 99$ & $19-22$ & --- & -- & 7.5 & --- & & $\cdots$ & $\cdots$ & Seep \\
\hline DV99-211 & Lower Ranch, upper spring & $05 / 09 / 99$ & 39.4 & -- & -- & 6.8 & --- & --- & --- & $\cdots$ & Flow $=10 \mathrm{l} / \mathrm{min}$ \\
\hline \multicolumn{12}{|c|}{ Background Wells } \\
\hline DV97-49 & Hole in the Wall \#2 Well & $11 / 04 / 97$ & 13.7 & $\cdots$ & --- & 6.0 & $-\cdots$ & $\cdots$ & --- & $\cdots$ & Unused well at abandoned wind mill \\
\hline DV97-57 & Bolivia Artesian Well & $11 / 05 / 97$ & 28.8 & --- & $\ldots$ & 7.0 & $\ldots$ & $\cdots$ & $\cdots$ & $\cdots$ & Flow $=40 \mathrm{l} / \mathrm{min}$ \\
\hline DV97-70 & Flowing well@AA Tank & $11 / 07 / 97$ & 16.7 & --- & & 6.2 & & --- & & --- & Flow $=5 \mathrm{l} / \mathrm{min}$ \\
\hline DV97-71 & Shaw Well & $11 / 07 / 97$ & 19.2 & --- & --- & 7.0 & --- & --- & $\cdots$ & --- & Flow $>60 \mathrm{l} / \mathrm{min}$ \\
\hline DV98-115 & Irrigation Well & $05 / 04 / 98$ & 17.8 & $\cdots$ & --- & 7.36 & $\cdots$ & -- & -- & $\ldots$ & Flow $\leq 100 \mathrm{gpm}$ pumped \\
\hline DV98-116 & Brinkerhoff Well & $05 / 04 / 98$ & 16.8 & --- & --- & 7.29 & --- & & --- & --- & Pumped: flow unknown \\
\hline DV98-172 & Bernice Well & $10 / 27 / 98$ & 16.5 & -.. & --- & 6.8 & --- & --- & --- & -.- & Unused well at building foundation \\
\hline \multicolumn{12}{|c|}{ Background Streams and Rain } \\
\hline DV97-58 & Cuttonwood Creek & $11 / 05 / 97$ & 14.0 & --- & --- & 7.0 & $\cdots$ & $\cdots$ & --- & --- & Flow moderated but not measured \\
\hline DV98-107 & Unnamed Ck by Stu's Seep & $04 / 30 / 98$ & 16.2 & --- & $\cdots$ & 8.0 & $\cdots$ & $\cdots$ & $--\cdot$ & ... & Below waterfall: flow moderate but not measured \\
\hline DV98-110 & Cottonwood Creck & $05 / 01 / 98$ & 20.2 & --- & --- & 8.0 & --- & & $\cdots$ & $-\cdots$ & Flow large but not measured \\
\hline DV98-119 & Unnamed Stream & $05 / 05 / 98$ & 23.4 & --- & --- & 8.50 & --- & $\cdots$ & $-\cdots$ & --- & Flow roughly $200 \mathrm{l} / \mathrm{min}$ \\
\hline DV98-121 & Whitc Rock Canyon & $05 / 05 / 98$ & 14.3 & --- & $\cdots$ & 8.80 & -- & $\cdots$ & $\cdots$ & $\cdots$ & Flow roughly $300 \mathrm{l} / \mathrm{min}$ \\
\hline DV98-125 & Rain, Lizard Well Tank & $05 / 06 / 98$ & 15.0 & $\cdots$ & --- & --- & --- & --- & -- & -- & \\
\hline DV98-126 & Home Station Wash & 05/06/98 & 19.6 & --- & $\cdots$ & 6.2 & $\cdots$ & $\cdots$ & --- & $\cdots$ & Flow roughly $2400 \mathrm{l} / \mathrm{min}$ (just rained) \\
\hline DV98-127 & Cedar Canyon Wash & $05 / 06 / 98$ & 18.6 & --- & & 6.5 & --- & -- & $\cdots$ & -- & Flow roughly 1600 l/min (just rained) \\
\hline DV98-130 & Bucher Creek & $05 / 06 / 98$ & 15.0 & --- & --- & --- & & --- & & --- & Flow roughly $200 \mathrm{~d} / \mathrm{min}$ \\
\hline DV98-171 & Not-So-OK Creek & $10 / 27 / 98$ & 10.0 & --- & --- & 6.8 & $\cdots$ & $\ldots$ & $\cdots$ & $\cdots$ & Flow roughly $1201 /$ min \\
\hline DV98-173 & Bernice Creck & $10 / 27 / 98$ & 13.4 & -- & --- & 7.2 & --- & --- & -- & -- & At Antimony King Mine; flow roughly $1201 /$ min \\
\hline DV98-174 & Hoyt Creek & $10 / 27 / 98$ & 12.8 & --- & --- & 8.5 & --- & $\cdots$ & --- & & Flow $=201 / \mathrm{min}$ \\
\hline DV98-180 & Mt. Augusta Creek & $10 / 28 / 98$ & 8.0 & --- & $\cdots$ & 6.5 & -- & --- & -.- & --- & Flow roughly $100 \mathrm{l} / \mathrm{min}$ \\
\hline DV99-213 & Dixie Salt Lake & $05 / 10 / 99$ & 19.2 & -.. & $\cdots$ & 8.2 & -- & $\cdots$ & --- & $\cdots$ & Near Dixie Hot Spring: salty precipitates \\
\hline
\end{tabular}

Two signilicant figures means $\mathrm{pH}$ was measured with paper: three significant tigures means $\mathrm{pH}$ wals meilsured with meter; number in parantheses gives hydroxide concentration in ppm (if mealsured).

"This temperature was obtained during nonflowing conditions: see DV98-111.

Two simples were mistakenly labcled 181: DV98-181 (Figure 8 Fumarole) and DV99-181 (Goerenger Well). 
Table 3: Analytical Methods Used for Water and Gas Analyses. Detection Limits (DLs) are in ppm unless noted."

\begin{tabular}{|c|c|c|c|c|c|c|c|c|c|}
\hline \multicolumn{7}{|c|}{ Methods For Waters } & \multicolumn{3}{|c|}{ Methods for gases } \\
\hline Analyte & Method & DL & Method & $\mathrm{DL}$ & Method & DL & Analyte & Method & $\mathrm{DL}$ \\
\hline $\mathrm{Ag}$ & GFAA & 0.0005 & ICP-AES & 0.002 & & & $\mathrm{Ar}$ & $\mathrm{GC}$ & $0.01 \%$ \\
\hline $\mathrm{Al}$ & GFAA & 0.002 & ICP-AES & 0.01 & & & As & Hydride AA & 0.002 \\
\hline Alkalinity & Calculation & & & & & & $\mathrm{Br}$ & $\mathrm{IC}$ & 0.2 \\
\hline As & Hydride-AA & 0.0002 & GFAA & 0.002 & ICP-AES & 0.05 & $\mathrm{C}_{2} \mathrm{H}_{6}$ & GC & $0.01 \%$ \\
\hline $\mathrm{Au}$ & GFAA & 0.002 & ICP-AES & 0.02 & & & $\mathrm{CH}_{+}$ & $\mathrm{GC}$ & $0.01 \%$ \\
\hline B & ICP-AES & 0.002 & & & & & $\mathrm{Cl}$ & IC & 1 \\
\hline $\mathrm{Ba}$ & ICP-AES & 0.002 & & & & & $\mathrm{CO}$ & $\mathrm{GC}$ & $0.01 \%$ \\
\hline $\mathrm{Be}$ & ICP-AES & 0.002 & & & & & $\mathrm{CO}_{2}$ & Titration & 5 \\
\hline $\mathrm{Br}$ & IC & 0.005 & & & & & $\mathrm{~F}$ & IC & 0.1 \\
\hline $\mathrm{Ca}$ & ICP-AES & 0.002 & & & & & $\mathrm{H}_{2}$ & $\mathrm{GC}$ & $0.01 \%$ \\
\hline $\mathrm{Cd}$ & GFAA & 0.0002 & ICP-AES & 0.005 & & & $\mathrm{H}_{2} \mathrm{~S}$ & ISE & 0.02 \\
\hline $\mathrm{Cl}$ & IC & 0.01 & & & & & $\mathrm{He}$ & GC & $0.01 \%$ \\
\hline Co & GFAA & 0.002 & ICP-AES & 0.01 & & & $\mathrm{Hg}$ & CVAA & 0.0002 \\
\hline $\mathrm{CO}_{3} / \mathrm{HCO}_{3} / \mathrm{OH}$ & Titration & 0.5 & & & & & $\mathrm{~N}_{2}$ & GC & $0.01 \%$ \\
\hline Conductivity & Electrode & 0.5 & & & & & $\mathrm{NH}_{3}$ & ISE & 0.05 \\
\hline $\mathrm{Cr}$ & GFAA & 0.002 & ICP-AES & 0.01 & & & $\mathrm{O}_{2}$ & $\mathrm{GC}$ & $0.01 \%$ \\
\hline Cs & GFAA & 0.002 & $\mathrm{AA}$ & 0.02 & & & $\mathrm{~S}$ & IC & 1 \\
\hline $\mathrm{Cu}$ & GFAA & 0.002 & ICP-AES & 0.01 & & & & & \\
\hline Eh & Electrode (in & & & & & & & & \\
\hline $\mathrm{F}$ & $\mathrm{IC}$ & 0.01 & Electrode & 0.01 & & & & & \\
\hline $\mathrm{Hg}$ & CVAA & 0.00005 & & & & & & & \\
\hline I & IC & 0.01 & & & & & & & \\
\hline K & $\mathrm{AA}$ & 0.01 & ICP-AES & 0.2 & & & & & \\
\hline $\mathrm{Li}$ & ICP-AES & 0.005 & & & & & & & \\
\hline $\mathrm{Mg}$ & ICP-AES & 0.002 & & & & & & & \\
\hline $\mathrm{Mn}$ & ICP-AES & 0.002 & & & & & & & \\
\hline Mo & GFAA & 0.002 & ICP-AES & 0.02 & & & & & \\
\hline $\mathrm{Na}$ & $\mathrm{AA}$ & 0.01 & ICP-AES & 0.05 & & & & & \\
\hline $\mathrm{NH}_{4}$ & Electrode & 0.02 & & & & & & & \\
\hline $\mathrm{Ni}$ & GFAA & 0.002 & ICP-AES & 0.01 & & & & & \\
\hline $\mathrm{NO}_{2}$ & IC & 0.01 & & & & & & & \\
\hline $\mathrm{NO}_{3}$ & IC & 0.01 & & & & & & & \\
\hline $\mathrm{Pb}$ & GFAA & 0.002 & ICP-AES & 0.05 & & & & & \\
\hline $\mathrm{pH}$ & Electrode & 0.01 & & & & & & & \\
\hline $\mathrm{PO}_{4}$ & IC & 0.02 & & & & & & & \\
\hline $\mathrm{Rb}$ & GFAA & 0.002 & AA & 0.01 & & & & & \\
\hline$S$ & Electrode & 0.02 & $\mathrm{IC}$ & 0.01 & & & & & \\
\hline $\mathrm{S}_{2} \mathrm{O}_{3}$ & IC & 0.01 & & & & & & & \\
\hline $\mathrm{Sb}$ & Hydride AA & 0.0002 & GFAA & 0.002 & ICP-AES & 0.05 & & & \\
\hline $\mathrm{Se}$ & Hydride AA & 0.0002 & GFAA & 0.002 & ICP-AES & 0.1 & & & \\
\hline $\mathrm{Si}$ & ICP-AES & 0.02 & & & & & & & \\
\hline $\mathrm{SO}_{3}$ & IC & 0.01 & & & & & & & \\
\hline $\mathrm{SO}_{4}$ & $\mathrm{IC}$ & 0.02 & & & & & & & \\
\hline $\mathrm{Sr}$ & ICP-AES & 0.005 & & & & & & & \\
\hline TDS & Calculation & & & & & & & & \\
\hline $\mathrm{Ti}$ & ICP-AES & 0.002 & & & & & & & \\
\hline $\mathrm{Tl}$ & GFAA & 0.002 & & & & & & & \\
\hline V & ICP-AES & 0.002 & & & & & & & \\
\hline $\mathrm{Zn}$ & ICP-AES & 0.005 & & & & & & & \\
\hline
\end{tabular}

"Methods used: AA = Atomic Adsorption Spectroscopy; CVAA = Cold Vapor AA; GFAA = Graphite Furnace AA; GC = Gas Chromatograpl IC = Ion Chromatography; ICP-AES = Inductively Coupled Plasma-Atomic Emission Spectroscopy; ISE = Ion Selective Electrode 
Table 4: Major Element Chemistry for Various Geothermal and Regional Fluids, Dixie Valley Region, Nevada (values in ppm unless otherwise noted).

\begin{tabular}{|c|c|c|c|c|c|c|c|c|c|c|c|c|c|c|}
\hline Sample & Name or Description & Date & $\begin{array}{c}\text { pH } \\
\text { (lab) }\end{array}$ & $\mathrm{SiO}_{2}$ & $\begin{array}{c}\mathrm{SiO}_{2}{ }^{\mathrm{a}} \\
\text { Method }\end{array}$ & $\mathrm{Na}$ & $\mathbf{K}$ & $\mathbf{L i}$ & $\mathrm{Ca}$ & Mg & $\mathbf{S r}$ & $\mathbf{F}$ & Cl & $\mathbf{B r}$ \\
\hline \multicolumn{15}{|l|}{ Brines } \\
\hline$\overline{D I X E 102-W}$ & V102+ V103 Separator & 10/02/95 & 9.45 & 638 & archived & 462 & 71.8 & 2.29 & 7.92 & 0.04 & 0.40 & 17.6 & 495 & 0.49 \\
\hline DV96-8 & 76-7 Well & $10 / 25 / 96$ & 9.09 & 599 & $\mathrm{TD}$ & 474 & 69.5 & 2.29 & 8.53 & 0.026 & 0.43 & 13.4 & 524 & 0.44 \\
\hline DV96-9 & V101 Separator & $10 / 25 / 96$ & 9.19 & 599 & TD & 407 & 64.0 & 2.03 & 8.03 & 0.007 & 0.37 & 15.5 & 438 & 0.32 \\
\hline DV97-11 & 73-7 Well & $10 / 29 / 97$ & 9.07 & 580 & FD & 508 & 74.4 & 2.45 & 8.96 & 0.02 & 0.45 & 14.3 & 594 & 0.65 \\
\hline DV97-13 & 84-7 Well & $10 / 29 / 97$ & 9.04 & 580 & FD & 496 & 70.8 & 2.46 & 9.66 & 0.01 & 0.46 & 13.5 & 558 & 0.61 \\
\hline DV97-14 & 74-7 Well & $10 / 29 / 97$ & 9.06 & 586 & FD & 500 & 72.2 & 2.43 & 9.20 & $<0.01$ & 0.49 & 13.5 & 584 & 0.66 \\
\hline DV97-16 & V102+ V103 Separator & $10 / 29 / 97$ & 9.04 & 586 & FD & 500 & 77.2 & 2.48 & 9.02 & $<0.01$ & 0.48 & 13.9 & 580 & 0.66 \\
\hline DV97-18 & V105 Separator & $10 / 29 / 97$ & 9.04 & 595 & FD & 502 & 73.5 & 2.29 & 9.53 & 0.02 & 0.46 & 14.3 & 574 & 0.72 \\
\hline DV97-20 & $82 \mathrm{~A}-7$ Well & $10 / 29 / 97$ & 9.05 & 556 & FD & 495 & 72.6 & 2.22 & 9.63 & $<0.01$ & 0.47 & 14.5 & 575 & 0.73 \\
\hline DV97-23 & 73B-7 Well & $10 / 30 / 97$ & 9.07 & 569 & FD & 499 & 76.4 & 2.34 & 9.09 & $<0.01$ & 0.45 & 13.7 & 571 & 0.64 \\
\hline DV $97-25$ & 27-33 Well & $10 / 30 / 97$ & 9.03 & 627 & FD & 423 & 66.8 & 2.22 & 7.69 & $<0.01$ & 0.32 & 14.7 & 443 & 0.56 \\
\hline DV97-26 & V101 Separator & 10/30/97 & 9.10 & 627 & FD & 439 & 68.8 & 2.27 & 7.95 & $<0.01$ & 0.34 & 15.4 & 463 & 0.55 \\
\hline DV97-29 & 37-33 Well & $10 / 30 / 97$ & 9.16 & 621 & FD & 431 & 68.8 & 2.26 & 7.20 & 0.02 & 0.32 & 16.1 & 475 & 0.41 \\
\hline DV97-30 & 28-33 Well & $10 / 30 / 97$ & 9.13 & 642 & FD & 429 & 70.1 & 2.24 & 7.40 & 0.02 & 0.34 & 15.4 & 470 & 0.61 \\
\hline DV98-73 & V101 Separator & $04 / 28 / 98$ & 9.10 & 591 & FD & 448 & 70.5 & 2.40 & 7.41 & 0.05 & 0.33 & 17.2 & 449 & 0.43 \\
\hline DV98-75 & 27-33 Well & $04 / 28 / 98$ & 8.99 & 571 & FD & 430 & 60.2 & 2.27 & 6.97 & $<0.01$ & 0.33 & 15.8 & 421 & 0.25 \\
\hline DV98-77 & 37-33 Well & $04 / 28 / 98$ & 9.03 & 554 & FD & 429 & 66.7 & 2.21 & 7.19 & $<0.01$ & 0.34 & 17.4 & 444 & 0.27 \\
\hline DV98-79 & 28-33 Well & $04 / 28 / 98$ & 9.01 & 550 & FD & 447 & 67.8 & 2.28 & 7.50 & $<0.01$ & 0.34 & 16.6 & 446 & 0.39 \\
\hline DV $98-80$ & 76A-7 Well & $04 / 28 / 98$ & 8.96 & 541 & FD & 498 & 75.6 & 2.58 & 8.56 & 0.01 & 0.42 & 14.1 & 556 & $<0.02$ \\
\hline DV98-82 & V102+ V103 Separator & $04 / 28 / 98$ & 8.94 & 518 & FD & 498 & 77.1 & 2.42 & 8.81 & 0.01 & 0.46 & 14.7 & 567 & 0.49 \\
\hline DV98-84 & 74-7 Well & $04 / 28 / 98$ & 8.99 & 531 & FD & 491 & 75.2 & 2.53 & 8.65 & 0.02 & 0.49 & 14.6 & 564 & 0.56 \\
\hline DV98-86 & 63-7 Well & $04 / 28 / 98$ & 8.97 & 516 & FD & 510 & 77.0 & 2.43 & 8.73 & $<0.01$ & 0.46 & 15.1 & 560 & 0.41 \\
\hline DV98-88 & 73-7 Well & $04 / 29 / 98$ & 8.98 & 518 & FD & 498 & 76.8 & 2.40 & 8.44 & 0.02 & 0.46 & 14.7 & 547 & 0.37 \\
\hline DV $98-90$ & 82A-7 Well & $04 / 29 / 98$ & 8.97 & 520 & FD & 501 & 76.1 & 2.22 & 8.95 & $<0.01$ & 0.42 & 15.6 & 561 & 0.41 \\
\hline DV98-92 & V105 Separator & $04 / 29 / 98$ & 8.96 & 526 & FD & 496 & 75.9 & 2.32 & 8.65 & $<0.01$ & 0.44 & 15.8 & 572 & 0.63 \\
\hline DV98-95 & 73B-7 Well & $04 / 29 / 98$ & 8.95 & 511 & $\mathrm{FD}$ & 500 & 74.2 & 2.27 & 8.43 & $<0.01$ & 0.44 & 15.7 & 561 & 0.54 \\
\hline DV98-133 & 27-33 Well & $10 / 20 / 98$ & 9.22 & 529 & FD & 381 & 61.8 & 2.11 & 4.85 & $<0.01$ & 0.24 & 14.4 & 405 & 0.28 \\
\hline DV98-135 & 27-33 Well & $10 / 20 / 98$ & 9.64 & 582 & FD & 467 & 60.0 & 2.61 & 9.46 & $<0.01$ & 0.51 & 18.0 & 496 & 0.40 \\
\hline DV98-138 & V10I Separator & $10 / 21 / 98$ & 9.35 & 546 & FD & 409 & 63.8 & 2.11 & 7.18 & $<0.01$ & 0.35 & 15.6 & 436 & 0.40 \\
\hline DV98-140 & 37-33 Well & $10 / 21 / 98$ & 9.35 & 526 & FD & 398 & 66.3 & 2.02 & 6.08 & 0.21 & 0.26 & 15.4 & 432 & 0.37 \\
\hline DV98-14I & 28-33 Well & $10 / 21 / 98$ & 9.38 & 531 & FD & 412 & 65.5 & 2.03 & 7.21 & 0.03 & 0.34 & 15.6 & 441 & 0.40 \\
\hline DV98-145 & 76A-7 Well & $10 / 22 / 98$ & 9.29 & 499 & FD & 479 & 70.8 & 2.26 & 8.23 & 0.01 & 0.44 & 13.7 & 541 & 0.44 \\
\hline DV98-147 & 63-7 Well & $10 / 22 / 98$ & 9.32 & 501 & FD & 496 & 71.9 & 2.21 & 8.87 & $<0.01$ & 0.45 & 14.6 & 565 & 0.55 \\
\hline
\end{tabular}


Table 4: Continued

Sample

DV98-148

DV98-150

DV98-152

DV98-154

DV98-156

DV98-159

DV99-182

DV99-184

DV99-186

DV99-188

DV99-190

DV99-194

DV99-196

DV99-197

DV99-199

N DV99-200

DV99-204

DV74782786-brine 2

DV76781986-brine 4

DV453382186-brine 6

DV73782886-brine 8

DV651882686-brine 12 65-18 Well Archived

No number

\section{Condensates}

DIXE102-S

DV96-7

DV96-10

DV97-12

DV97-15

DV97-17

DV97-19

DV97-21
28-33 Well Archived

\section{Name or Description}

V102+V103 Separator

7 Well

73B-7 Wel

82A-7 Well

V 105 Separator

76A-7 Well

V102 + V103 Separator

63-7 Well

73-7 Well

V105 Separator

82A-7 Wel

28-33 Well

V101 Separator

74-7 Well Archived

6-7 Well Archived

45-33 Well Archived

V $102+$ V 103 Separator

76-7 Well

V101 Separator

73-7 Well

74-7 Well

V102+V103 Separator

V105 Separator

82A-7 Well

\begin{tabular}{|c|c|c|c|c|}
\hline Date & $\begin{array}{l}\text { pH } \\
\text { (lab) }\end{array}$ & $\mathrm{SiO}_{2}$ & $\begin{array}{c}\mathrm{SiO}_{2}{ }^{2} \\
\text { Method }\end{array}$ & $\mathrm{Na}$ \\
\hline $10 / 22 / 98$ & 9.31 & 514 & FD & 485 \\
\hline $10 / 22 / 98$ & 9.30 & 518 & FD & 486 \\
\hline $10 / 22 / 98$ & 9.32 & 509 & FD & 476 \\
\hline $10 / 22 / 98$ & 9.28 & 514 & FD & 485 \\
\hline $10 / 23 / 98$ & 9.30 & 514 & FD & 473 \\
\hline $10 / 23 / 98$ & 9.30 & 507 & FD & 480 \\
\hline $05 / 04 / 99$ & 9.02 & 524 & FA & 508 \\
\hline $05 / 04 / 99$ & 9.00 & 522 & FA & 482 \\
\hline 05/04/99 & 9.01 & 522 & FA & 496 \\
\hline 05/04/99 & 9.01 & 516 & $\mathrm{FA}$ & 504 \\
\hline $05 / 04 / 99$ & 9.01 & 518 & FA & 508 \\
\hline $05 / 05 / 99$ & 8.98 & 514 & FA & 514 \\
\hline $05 / 05 / 99$ & 9.01 & 503 & FA & 518 \\
\hline $05 / 05 / 99$ & 9.00 & 520 & FA & 516 \\
\hline $05 / 05 / 99$ & 9.05 & 563 & $\mathrm{FA}$ & 433 \\
\hline $05 / 05 / 99$ & 9.10 & 561 & FA & 432 \\
\hline $05 / 05 / 99$ & 9.05 & 576 & FA & 428 \\
\hline $08 / 27 / 86$ & 9.13 & 574 & archived & 413 \\
\hline $08 / 19 / 86$ & 9.16 & 563 & archived & 403 \\
\hline $08 / 21 / 86$ & 9.12 & 589 & archived & 370 \\
\hline $08 / 28 / 86$ & 9.01 & 548 & archived & 380 \\
\hline $08 / 26 / 86$ & 8.70 & 484 & archived & 406 \\
\hline $08 / 26 / 86$ & 8.85 & 417 & archived & 440 \\
\hline $09 / 23 / 93$ & 7.58 & 101 & archived & 228 \\
\hline
\end{tabular}

\section{K}

Li

Ca

$72.3 \quad 2.24$

$\begin{array}{llllllll}72.0 & 2.15 & 7.75 & 0.01 & 0.42 & 14.2 & 560 & 0.59\end{array}$

$\begin{array}{lllllllll}69.3 & 2.10 & 8.87 & 0.04 & 0.46 & 14.7 & 557 & 0.55\end{array}$

$\begin{array}{llllllll}69.4 & 2.14 & 9.39 & 0.34 & 0.48 & 14.4 & 560 & 0.59\end{array}$

$\begin{array}{llllllll}73.7 & 2.51 & 8.52 & 0.24 & 0.43 & 14.0 & 576 & 0.64\end{array}$

$\begin{array}{lllllllll}74.3 & 2.32 & 8.65 & 0.33 & 0.46 & 15.1 & 592 & 0.47\end{array}$

$\begin{array}{llllllll}72.7 & 2.33 & 8.47 & <0.01 & 0.46 & 15.2 & 594 & 0.59\end{array}$

$\begin{array}{lllllllll}73.6 & 2.26 & 8.48 & <0.01 & 0.45 & 15.5 & 604 & 0.49\end{array}$

$\begin{array}{llllllll}74.6 & 2.21 & 8.79 & <0.01 & 0.45 & 15.5 & 624 & 0.64\end{array}$

$\begin{array}{lllllllll}74.4 & 2.23 & 9.51 & <0.01 & 0.47 & 15.9 & 620 & 0.58\end{array}$

$\begin{array}{llllllll}72.2 & 2.25 & 8.89 & <0.01 & 0.47 & 16.0 & 623 & 0.58\end{array}$

$\begin{array}{lllllllll}74.4 & 2.22 & 8.78 & <0.01 & 0.47 & 15.9 & 624 & 0.44\end{array}$

$\begin{array}{llllllll}65.7 & 2.23 & 6.66 & 0.12 & 0.31 & 16.0 & 475 & 0.38\end{array}$

$\begin{array}{llllllll}66.2 & 2.24 & 6.68 & 0.02 & 0.32 & 16.3 & 483 & 0.50\end{array}$

$\begin{array}{llllllll}68.4 & 2.39 & 7.35 & <0.01 & 0.31 & 16.4 & 481 & 0.34\end{array}$

$\begin{array}{lllllllll}61.5 & 2.82 & 1.11 & <0.01 & 0.16 & 11.3 & 396 & 0.40\end{array}$

$\begin{array}{llllllll}54.2 & 2.79 & 1.53 & <0.01 & 0.17 & 10.1 & 402 & 0.31\end{array}$

$\begin{array}{lllllllll}59.2 & 2.63 & 1.27 & 0.04 & 0.13 & 14.9 & 320 & 0.30\end{array}$

$\begin{array}{llllllll}59.2 & 2.55 & 1.24 & <0.01 & 0.15 & 10.6 & 363 & 0.39\end{array}$

$\begin{array}{llllllll}43.3 & 2.65 & 2.07 & <0.01 & 0.23 & 8.36 & 428 & 0.37\end{array}$

$\begin{array}{llllllll}40.7 & 2.09 & 1.16 & <0.01 & 0.21 & 7.17 & 404 & 0.37\end{array}$

$\begin{array}{llllllll}6.13 & 0.35 & 15.6 & 2.08 & 1.33 & 4.28 & 70.1 & 0.19\end{array}$

$10 / 02 / 95 \quad 5.85$

$10 / 25 / 96 \quad 5.98$

$10 / 25 / 96 \quad 5.98$

$10 / 29 / 97 \quad 5.29$

$10 / 29 / 97 \quad 4.93$

$10 / 29 / 97 \quad 4.99$

$0 / 29 / 97 \quad 5.24$

$10 / 29 / 97 \quad 5.20$
4.6

4.2

$--$

$---$

$--$
2.7 archived 2.74

列

$\begin{array}{lllllllll}--- & -- & -.- & \ldots & -. & \ldots & 0.07 & 3.1 & <0.02\end{array}$

$\begin{array}{llllllll}1.03 & <0.01 & 0.15 & <0.01<0.01 & 0.03 & 0.26 & <0.02\end{array}$ $\begin{array}{llllllll}1.33 & <0.01 & 0.22 & 0.01 & <0.01 & 0.01 & 0.13 & <0.02\end{array}$

$\begin{array}{lllllllll}28.6 & -- & -- & --- & -- & 6 & 242 & 0.32\end{array}$

$\begin{array}{llllllll}3.21 & -- & --- & -- & -- & 0.14 & 7.26 & <0.02\end{array}$

-- $---\quad---\quad---\quad<0.01<0.02<0.02$

$\begin{array}{lllllll}-- & -- & -- & -- & <0.01 & 0.31<0.02\end{array}$

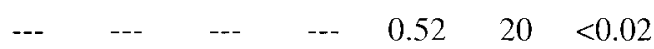


Table 4: Continued

\begin{tabular}{|c|c|c|c|c|c|c|c|c|c|c|c|c|c|c|}
\hline Sample & Name or Description & Date & $\begin{array}{c}\mathbf{p H} \\
(\mathbf{l a b})\end{array}$ & $\mathrm{SiO}_{2}$ & $\begin{array}{c}\mathrm{SiO}_{2}^{\mathrm{a}} \\
\text { Method }\end{array}$ & $\mathbf{N a}$ & $\mathbf{K}$ & $\mathbf{L i}$ & $\mathbf{C a}$ & Mg & $\mathbf{S r}$ & $\mathbf{F}$ & Cl & $\mathrm{Br}$ \\
\hline DV97-22 & 73B-7 Well & $10 / 29 / 97$ & 5.73 & --- & --- & 234 & 37.2 & --- & --- & --- & -- & 6.37 & 281 & 0.3 \\
\hline DV97-24 & V101 Separator & $10 / 30 / 97$ & 4.96 & $--\cdot$ & --- & 0.28 & 0.48 & --- & --- & --- & --- & 0.05 & $<0.02$ & $<0.02$ \\
\hline DV97-27 & 27-33 Well & $10 / 30 / 97$ & 5.37 & --- & --- & 1.51 & 0.8 & --- & --- & --- & --- & 0.06 & 0.87 & $<0.02$ \\
\hline DV97-28 & 37-33 Well & $10 / 30 / 97$ & 5.35 & --- & --- & 0.07 & 0.05 & --- & -- & --- & --- & $<0.01$ & $<0.02$ & $<0.02$ \\
\hline DV97-31 & 28-33 Well & $10 / 30 / 97$ & 5.12 & --- & --- & 6.39 & 1.94 & --- & -- & $\cdots$ & --- & 0.12 & 3.69 & $<0.02$ \\
\hline DV98-74 & V101 Separator & $04 / 28 / 98$ & 5.34 & 2.2 & $\mathrm{~T}$ & 0.64 & 0.75 & $<0.01$ & 0.13 & $<0.01$ & --- & 0.03 & 0.07 & $<0.02$ \\
\hline DV98-76 & 27-33 Well & $04 / 28 / 98$ & 5.66 & 4.8 & $\mathrm{~T}$ & 0.35 & 0.29 & $<0.01$ & 0.10 & $<0.01$ & $\cdots$ & 0.01 & 0.09 & $<0.02$ \\
\hline DV98-78 & 37-33 Well & $04 / 28 / 98$ & 5.54 & 5.1 & $\mathrm{~T}$ & 0.57 & 0.34 & 0.01 & 1.07 & 0.06 & --- & 0.02 & 0.21 & $<0.02$ \\
\hline DV98-81 & 76A-7 Well & $04 / 28 / 98$ & 5.60 & 0.8 & $\mathrm{~T}$ & 0.08 & 0.16 & $<0.01$ & 0.05 & $<0.01$ & --- & $<0.01$ & $<0.02$ & $<0.02$ \\
\hline DV98-83 & V102 + V103 Separator & $04 / 28 / 98$ & 5.87 & 0.5 & $\mathrm{~T}$ & 0.93 & 0.73 & $<0.01$ & 0.14 & 0.02 & --- & $<0.01$ & 0.20 & $<0.02$ \\
\hline DV98-85 & 74-7 Well & $04 / 28 / 98$ & 5.58 & 15.1 & $\mathrm{~T}$ & 13.2 & 2.23 & 0.05 & 0.13 & $<0.01$ & --- & 0.35 & 15.7 & $<0.02$ \\
\hline DV98-87 & 63-7 Well & $04 / 28 / 98$ & 5.63 & 1.8 & $\mathrm{~T}$ & 0.30 & 0.13 & $<0.01$ & 0.17 & $<0.01$ & --- & $<0.01$ & 0.10 & $<0.02$ \\
\hline DV98-89 & 73-7 Well & $04 / 29 / 98$ & 6.03 & 342 & $\mathrm{~T}$ & 344 & 51.5 & 1.63 & 5.04 & 0.01 & --- & 10.2 & 388 & 0.30 \\
\hline DV98-91 & 82A-7 Well & $04 / 29 / 98$ & 5.94 & 244 & $\mathrm{~T}$ & 246 & 34.5 & 1.09 & 4.13 & $<0.01$ & --- & 7.48 & 281 & 0.23 \\
\hline DV98-93 & V105 Separator & $04 / 29 / 98$ & 5.70 & 0.6 & $\mathrm{~T}$ & 0.29 & 0.26 & $<0.01$ & 0.05 & $<0.01$ & --- & $<0.01$ & 0.03 & $<0.02$ \\
\hline DV98-94 & 73B-7 Well & $04 / 29 / 98$ & 5.85 & 1.0 & $\mathrm{~T}$ & 0.93 & 0.33 & $<0.01$ & 0.09 & $<0.01$ & --- & 0.05 & 0.45 & $<0.02$ \\
\hline DV98-101 & 28-33 Well & $04 / 30 / 98$ & 5.78 & 1.2 & $\mathrm{~T}$ & 0.85 & 0.33 & $<0.01$ & 0.06 & 0.01 & --- & 0.03 & 0.45 & $<0.02$ \\
\hline DV98-136 & 27-33 Well & $10 / 20 / 98$ & 5.96 & 0.7 & $\mathrm{~T}$ & 0.86 & 0.51 & $<0.01$ & 0.12 & 0.02 & --- & 0.02 & 0.50 & $<0.02$ \\
\hline DV98-137 & V101 Separator & $10 / 21 / 98$ & 5.93 & 0.3 & $\mathrm{~T}$ & 0.06 & 0.07 & $<0.01$ & 0.05 & $<0.01$ & --- & $<0.01$ & $<0.02$ & $<0.02$ \\
\hline DV98-139 & 37-33 Well & $10 / 21 / 98$ & 5.75 & 0.6 & $\mathrm{~T}$ & 0.45 & 0.47 & $<0.01$ & 0.04 & $<0.01$ & --- & $<0.01$ & 0.04 & $<0.02$ \\
\hline DV98-142 & 28-33 Well & $10 / 21 / 98$ & 5.69 & 2.2 & $\mathrm{~T}$ & 1.06 & 0.50 & $<0.01$ & 1.14 & 0.07 & --- & 0.03 & 0.62 & $<0.02$ \\
\hline DV98-144 & 76A-7 Well & $10 / 22 / 98$ & 6.14 & $<0.05$ & $\mathrm{~T}$ & 0.79 & 0.73 & $<0.01$ & 0.08 & $<0.01$ & --- & $<0.01$ & 0.03 & $<0.02$ \\
\hline DV98-146 & V102 + V103 Separator & $10 / 22 / 98$ & 6.33 & $<0.05$ & $\mathrm{~T}$ & 0.08 & 0.07 & $<0.01$ & 0.08 & 0.01 & --- & $<0.01$ & 0.02 & $<0.02$ \\
\hline DV98-149 & 63-7 Well & $10 / 22 / 98$ & 5.50 & 0.5 & $\mathbf{T}$ & 0.03 & $<0.01$ & $<0.01$ & 0.09 & $<0.01$ & --- & $<0.01$ & 0.03 & $<0.02$ \\
\hline DV98-151 & 74-7 Well & $10 / 22 / 98$ & 5.71 & 15.3 & $\mathrm{~T}$ & 13.4 & 2.18 & 0.06 & 0.26 & 0.01 & --- & 0.40 & 14.9 & $<0.02$ \\
\hline DV98-153 & 73-7 Well & $10 / 22 / 98$ & 5.90 & 81.5 & $\mathrm{~T}$ & 86.9 & 12.5 & 0.37 & 1.47 & 0.31 & --- & 2.65 & 105 & 0.09 \\
\hline DV98-155 & 73B-7 Well & $10 / 22 / 98$ & 6.15 & 248 & $\mathrm{~T}$ & 239 & 33.2 & 0.99 & 3.65 & 0.03 & --- & 7.31 & 287 & 0.24 \\
\hline DV98-157 & 82A-7 Well & $10 / 23 / 98$ & 5.67 & 80.5 & $\mathrm{~T}$ & 83.4 & 12.1 & 0.35 & 1.24 & 0.03 & --- & 2.48 & 90.9 & 0.07 \\
\hline DV98-158 & V105 Separator & $10 / 23 / 98$ & 5.79 & 0.4 & $\mathrm{~T}$ & 0.29 & 0.24 & $<0.01$ & 0.05 & $<0.01$ & $\cdots$ & $<0.01$ & 0.03 & $<0.02$ \\
\hline DV99-183 & 76A-7 Well & 05/04/99 & 6.66 & 3.36 & $\mathrm{~T}$ & 0.2 & 0.38 & $<0.01$ & 0.11 & 0.05 & --- & 0.01 & 0.08 & $<0.01$ \\
\hline DV99-185 & 74-7 Well & $05 / 04 / 99$ & 6.24 & 56.3 & $\mathrm{~T}$ & 54.8 & 7.94 & 0.26 & 0.84 & $<0.01$ & --- & 1.46 & 58 & 0.04 \\
\hline DV99-187 & V $102+V 103$ Separator & $05 / 04 / 99$ & 6.52 & 2.95 & $\mathrm{~T}$ & 1.25 & 2.14 & $<0.01$ & 0.08 & $<0.01$ & --- & $<0.01$ & 0.02 & $<0.01$ \\
\hline DV99-189 & 63-7 Well & $05 / 04 / 99$ & 6.59 & 2.63 & $\mathrm{~T}$ & 0.33 & 0.51 & $<0.01$ & 0.02 & $<0.01$ & --- & $<0.01$ & 0.16 & $<0.01$ \\
\hline DV99-191 & 73-7 Well & $05 / 04 / 99$ & 6.53 & 114 & $\mathrm{~T}$ & 117 & 17.3 & 0.51 & 1.49 & $<0.01$ & --- & 3.29 & 132 & 0.1 \\
\hline
\end{tabular}




\section{Table 4: Continued}

\begin{tabular}{|c|c|c|c|c|c|c|c|c|c|c|c|c|c|c|}
\hline Sample & Name or Description & Date & $\begin{array}{c}\mathbf{p H} \\
(\text { lab) }\end{array}$ & $\mathrm{SiO}_{2}$ & $\begin{array}{c}\mathrm{SiO}_{2}{ }^{\mathrm{a}} \\
\text { Method }\end{array}$ & $\mathbf{N a}$ & $\mathbf{K}$ & $\mathbf{L i}$ & Ca & Mg & $\mathbf{S r}$ & $\mathbf{F}$ & Cl & $\mathbf{B r}$ \\
\hline DV99-192 & 73B-7 Well & $05 / 04 / 99$ & 6.60 & 166 & $\mathrm{~T}$ & 164 & 22.5 & 0.74 & 1.80 & $<0.01$ & --- & 5.42 & 181 & 0.13 \\
\hline DV99-193 & V105 Separator & $05 / 05 / 99$ & 5.95 & 2.40 & $\mathrm{~T}$ & 0.79 & 1.79 & $<0.01$ & 0.08 & $<0.01$ & --- & 0.01 & 0.03 & $<0.01$ \\
\hline DV99-195 & 82A-7 Well & $05 / 05 / 99$ & 6.59 & 159 & $\mathrm{~T}$ & 158 & 19.3 & 0.68 & 2.91 & $<0.01$ & --- & 4.82 & 175 & 0.13 \\
\hline DV99-201 & 28-33 Well & $05 / 05 / 99$ & 6.16 & 6.16 & $\mathrm{~T}$ & 2.77 & 2.36 & $<0.01$ & 0.12 & $<0.01$ & --- & 0.02 & 0.91 & $<0.01$ \\
\hline DV99-202 & 37-33 Well & $05 / 05 / 99$ & 5.67 & 2.14 & $\mathrm{~T}$ & 0.6 & 0.29 & $<0.01$ & 0.27 & $<0.01$ & --- & $<0.01$ & 0.43 & $<0.01$ \\
\hline DV99-203 & V101 Separator & 05/05/99 & 6.50 & 1.95 & $\mathrm{~T}$ & 0.16 & 0.14 & $<0.01$ & 0.02 & $<0.01$ & --- & $<0.01$ & 0.17 & $<0.01$ \\
\hline DV74782786-cond 1 & 74-7 Well Archived & $08 / 27 / 86$ & 7.13 & 3.77 & archived & 3.79 & 0.01 & 0.03 & 0.94 & $<0.01$ & --- & $<0.01$ & 0.04 & $<0.01$ \\
\hline DV76781986-cond 3 & 76-7 Well Archived & $08 / 19 / 86$ & 6.97 & 5.39 & archived & 3.79 & 0.02 & $<0.01$ & 0.33 & $<0.01$ & --- & $<0.01$ & 0.2 & $<0.01$ \\
\hline DV453382886-cond 5 & 45-33 Well Archived & $08 / 28 / 86$ & 7.00 & 5.11 & archived & 3.82 & 0.03 & $<0.01$ & 0.38 & $<0.01$ & --- & $<0.01$ & 0.15 & $<0.01$ \\
\hline DV73782886-cond 7 & 73-7 Well Archived & $08 / 28 / 86$ & 6.78 & 5.09 & archived & 4 & 0.02 & $<0.01$ & 0.36 & $<0.01$ & --- & $<0.01$ & 0.44 & $<0.01$ \\
\hline DV321882686-cond 9 & 32-18 Well Archived & $08 / 26 / 86$ & 6.82 & 5.29 & archived & 3.94 & 0.02 & $<0.01$ & 0.38 & $<0.01$ & --- & $<0.01$ & 0.13 & $<0.01$ \\
\hline DV651882686-cond 11 & 65-18 Well Archived & $08 / 26 / 86$ & 7.03 & 3.21 & archived & 3.78 & $<0.01$ & $<0.01$ & 0.67 & $<0.01$ & --- & $<0.01$ & 0.04 & $<0.01$ \\
\hline \multicolumn{15}{|c|}{ Injection Well/Power Plant Fluids } \\
\hline DV96-2 & Condensate from plant & $10 / 24 / 96$ & 6.63 & 1.7 & FA & 0.65 & 0.11 & $<0.01$ & 1.3 & 0.12 & 0.01 & 0.01 & 0.42 & $<0.02$ \\
\hline DV96-3 & LPBrine @ Plant & $10 / 24 / 96$ & 9.61 & 644 & TD & 493 & 73.4 & 2.61 & 8.74 & 0.027 & 0.41 & 17.8 & 519 & 0.409 \\
\hline DV96-4 & 45-5 Injection Well & $10 / 24 / 96$ & 9.54 & 601 & TD & 470 & 72.0 & 2.50 & 8.38 & 0.023 & 0.41 & 15.0 & 518 & 0.368 \\
\hline DV96-5 & Lamb 1 Injection Well & $10 / 24 / 96$ & 9.59 & 618 & $\mathrm{TD}$ & 506 & 74.6 & 2.52 & 9.55 & 0.014 & 0.46 & 16.2 & 549 & 0.442 \\
\hline DV96-6 & 65-18 Injection Well & $10 / 24 / 96$ & 9.58 & 629 & TD & 506 & 75.2 & 2.57 & 8.68 & 0.009 & 0.45 & 16.1 & 556 & 0.451 \\
\hline DV97-32 & Condensate from plant & $10 / 31 / 97$ & 6.22 & --- & --- & 56.4 & 7.83 & --- & --- & --- & --- & 1.46 & 62.2 & 0.06 \\
\hline DV97-33 & LP Brine & $10 / 31 / 97$ & 9.49 & 655 & $\mathrm{FD}$ & 483 & 72.6 & 2.62 & 8.85 & $<0.01$ & 0.46 & 16.1 & 579 & 0.58 \\
\hline DV97-34 & $25-5+45-5$ Injectate & $10 / 31 / 97$ & 9.40 & 574 & FD & 434 & 66.4 & 2.24 & 7.76 & 0.03 & 0.39 & 14.4 & 515 & 0.54 \\
\hline DV97-35 & $25-5+45-5$ Injectate & $10 / 31 / 97$ & 9.42 & 561 & FD & 450 & 67.6 & 2.29 & 7.93 & 0.03 & 0.39 & 14.4 & 511 & 0.47 \\
\hline DV97-36 & 65-18 Injection Well & $10 / 31 / 97$ & 9.50 & 629 & FD & 497 & 74.0 & 2.61 & 8.65 & 0.01 & 0.45 & 16.6 & 590 & 0.53 \\
\hline DV97-37 & 32-18 Injection Well & $10 / 31 / 97$ & 9.49 & 597 & FD & 499 & 76.8 & 2.53 & 8.95 & $<0.01$ & 0.47 & 16.3 & 588 & 0.54 \\
\hline DV97-40 & LPBrine@Plant & $10 / 31 / 97$ & 9.49 & 642 & FD & 465 & 72.5 & 2.66 & 9.03 & 0.03 & 0.46 & 15.6 & 571 & 0.58 \\
\hline DV97-41 & Condensate from plant & $10 / 31 / 97$ & 6.66 & --- & --- & 56.1 & 7.78 & --- & --- & --- & --- & 1.48 & 63.1 & $<0.02$ \\
\hline DV97-42 & High P Brine @ plant & $10 / 31 / 97$ & 9.09 & 593 & FD & 432 & 70.0 & 2.28 & 7.25 & 0.03 & 0.34 & 15.4 & 464 & 0.49 \\
\hline DV98-97 & Condensate from plant & $04 / 29 / 98$ & 6.95 & 1.1 & $\mathrm{~T}$ & 1.17 & 0.20 & $<0.01$ & 0.65 & 0.15 & --- & 0.04 & 0.70 & $<0.02$ \\
\hline DV98-98 & LPBrine @ Plant & $04 / 29 / 98$ & 9.38 & 597 & FD & 536 & 79.9 & 2.61 & 9.69 & 0.17 & 0.46 & 18.0 & 589 & 0.55 \\
\hline DV98-143 & 25-5 Injection Well & $10 / 21 / 98$ & 9.72 & 561 & FD & 508 & 74.5 & 2.33 & 9.03 & 0.01 & 0.45 & 16.4 & 576 & 0.52 \\
\hline DV98-161 & Condensate from plant & $10 / 23 / 98$ & 7.16 & 25.7 & FA & 33.3 & 4.54 & 0.15 & 2.56 & 1.20 & 0.059 & 0.89 & 33.0 & 0.02 \\
\hline DV98-162 & LPBrine @ Plant & $10 / 23 / 98$ & 9.74 & 599 & FD & 510 & 75.8 & 2.46 & 9.63 & 0.12 & 0.47 & 16.2 & 573 & 0.41 \\
\hline DV98-163 & 65-18 Injection Well & $10 / 23 / 98$ & 8.04 & 43.2 & FD & 134 & 12.3 & 0.48 & 28.7 & 23.5 & 0.61 & 1.02 & 146 & 0.09 \\
\hline
\end{tabular}


Table 4: Continued

\begin{tabular}{|c|c|c|c|c|c|c|c|c|c|c|c|c|c|c|}
\hline Sample & Name or Description & Date & $\begin{array}{c}\text { pH } \\
\text { (lab) }\end{array}$ & $\mathrm{SiO}_{2}$ & $\begin{array}{c}\mathrm{SiO}_{2}^{\mathrm{a}} \\
\text { Method }\end{array}$ & $\mathbf{N a}$ & $\mathbf{K}$ & $\mathbf{L i}$ & $\mathbf{C a}$ & Mg & $\mathbf{S r}$ & $\mathbf{F}$ & $\mathrm{Cl}$ & $\mathbf{B r}$ \\
\hline DV99-198 & 65-18 Injection Well & $05 / 05 / 99$ & 8.01 & 38.5 & FA & 151 & 12.8 & 0.57 & 32.7 & 27.5 & 0.71 & 0.61 & 166 & 0.12 \\
\hline DV99-205 & $25-5+45-5$ Injectate & $05 / 06 / 99$ & 9.43 & 603 & FA & 539 & 80.5 & 2.63 & 9.08 & $<0.01$ & 0.45 & 17.9 & 631 & 0.78 \\
\hline DV99-206 & LPBrine@ @lant & $05 / 06 / 99$ & 9.44 & 603 & FA & 528 & 79.7 & 2.55 & 8.70 & $<0.01$ & 0.46 & 17.2 & 634 & 0.63 \\
\hline DV99-207 & Condensate from plant & $05 / 06 / 99$ & 7.36 & 2.78 & FA & 1.06 & 0.33 & $<0.01$ & 0.12 & $<0.01$ & 0.004 & $<0.01$ & 0.45 & $<0.02$ \\
\hline DV99-208 & $52-18+41-18$ Injectate & 05/06/99 & 9.41 & 595 & FA & 529 & 78.5 & 2.60 & 8.80 & $<0.01$ & 0.44 & 17.0 & 621 & 0.63 \\
\hline \multicolumn{15}{|c|}{ Other Geothermal and On-Site Water Wells } \\
\hline DV96-1 & Domestic Well & $10 / 24 / 96$ & & 74.3 & FA & 143 & 15.4 & 0.43 & 61.6 & 32.0 & 1.63 & 0.78 & 105 & 0.12 \\
\hline DV97-38 & Domestic Well & $10 / 31 / 97$ & 7.82 & 77.0 & FA & 136 & 16.3 & 0.42 & 58.7 & 28.8 & 1.66 & 0.76 & 109 & 0.15 \\
\hline DV97-39 & Goerenger Well & $10 / 31 / 97$ & 7.70 & 62.1 & FA & 228 & 20.4 & 0.9 & 50.3 & 37.5 & 1.06 & 1.21 & 222 & 0.26 \\
\hline DV97-53 & 46-32 Well & $11 / 05 / 97$ & 5.93 & 3.9 & $\mathrm{~T}$ & 4.17 & 2.26 & 0.02 & 0.83 & 0.04 & 0.13 & 0.09 & 2.64 & $<0.02$ \\
\hline DV97-54 & 27-32 Well & $11 / 05 / 97$ & 6.22 & 3.0 & $\mathrm{~T}$ & 0.31 & 0.19 & 0.01 & 0.18 & 0.01 & 0.003 & $<0.02$ & 0.27 & $<0.02$ \\
\hline DV97-55 & 27-32 Well & $11 / 05 / 97$ & 6.29 & 58.2 & FA & 95.5 & 13.1 & 0.7 & 5.56 & 0.03 & 0.21 & 5.44 & 87.6 & 0.06 \\
\hline DV97-59 & 45-W-5 Well & $11 / 05 / 97$ & 7.75 & 22.5 & $\mathrm{FA}$ & 204 & 8.53 & 0.68 & 4.85 & 3.02 & 0.19 & 1.95 & 201 & 0.23 \\
\hline DV97-67 & 66-21 Well & $11 / 07 / 97$ & 6.97 & 321 & FA & 935 & 86.6 & 4.57 & 41.2 & 0.41 & 2.48 & 2.68 & 1476 & 1.32 \\
\hline DV98-96 & Goerenger Well & $04 / 29 / 98$ & 7.88 & 61.4 & FA & 254 & 22.1 & 0.92 & 52.3 & 42.5 & 1.11 & 1.29 & 218 & 0.21 \\
\hline DV98-99 & 27-32 Well & $04 / 29 / 98$ & 5.81 & 61.2 & $\mathrm{~T}$ & 88.0 & 11.6 & 0.48 & 5.91 & 0.91 & --- & 4.00 & 84.8 & 0.05 \\
\hline DV98-100 & 46-32 Well & $04 / 29 / 98$ & 5.72 & 0.8 & $\mathrm{~T}$ & 0.69 & 0.74 & 0.01 & 0.30 & 0.06 & --- & $<0.01$ & 0.24 & $<0.02$ \\
\hline DV98-102 & 45-14 Well & $04 / 30 / 98$ & 5.71 & 19.8 & $\mathbf{T}$ & 31.0 & 2.83 & 0.07 & 1.93 & 0.02 & --- & 0.58 & 36.0 & $<0.02$ \\
\hline DV98-103 & 45-14 Well & $04 / 30 / 98$ & 7.23 & 285 & FD & 432 & 41.4 & 1.06 & 23.0 & 0.04 & 1.10 & 7.92 & 481 & 0.49 \\
\hline DV98-104 & 66-21 Well & $04 / 30 / 98$ & 6.51 & 325 & $\mathrm{FD}$ & 876 & 86.9 & 4.89 & 40.0 & 0.35 & 2.61 & 3.06 & 1440 & 1.05 \\
\hline DV98-111 & 62-21 Well & $05 / 01 / 98$ & 7.84 & 172 & $\mathrm{FD}$ & 513 & 17.1 & 0.49 & 6.10 & 0.41 & 0.48 & 6.60 & 79.6 & 0.15 \\
\hline DV98-122 & 97-2 Well & $05 / 05 / 98$ & 7.96 & 55.2 & $\mathrm{FA}$ & 383 & 35.3 & 1.45 & 52.6 & 47.5 & 1.10 & 3.73 & 325 & 0.25 \\
\hline DV98-123 & 32-6 Well & $05 / 06 / 98$ & 8.15 & 2.5 & FA & 179 & 6.83 & 0.07 & 28.1 & 64.1 & 0.31 & 1.55 & 189 & 0.20 \\
\hline Dixie Jack \#l & Gradient Well DJ \#I & $05 / 17 / 98$ & 7.93 & 43.2 & FA & 410 & 25.9 & 1.65 & 37.8 & 5.30 & 0.38 & 7.55 & 316 & 0.26 \\
\hline Dixie Jack \#4 & Gradient Well DJ \#4 & $05 / 20 / 98$ & 7.45 & 119 & FA & 281 & 24.7 & 1.17 & 12.2 & 0.57 & 0.34 & 20.4 & 282 & 0.21 \\
\hline Dixie Jack \#7 & Gradient Well DJ \#7 & $05 / 14 / 98$ & 7.13 & 5.0 & FA & 19.9 & 2.12 & 0.09 & 4.21 & 0.18 & 0.10 & 0.72 & 6.20 & $<0.02$ \\
\hline DV98-160 & Goerenger Well & $10 / 23 / 98$ & 8.18 & 59.7 & FD & 231 & 20.6 & 0.80 & 52.2 & 43.8 & 1.17 & 1.18 & 252 & 0.21 \\
\hline DV98-168 & 38-32 Well & $10 / 26 / 98$ & 8.22 & 166 & FA & 234 & 25.2 & 0.98 & 14.4 & 1.08 & 0.47 & 15.9 & 161 & 0.14 \\
\hline DV98-175 & $62-21$ Well & $10 / 28 / 98$ & 7.86 & 162 & FA & 488 & 16.5 & 0.42 & 12.1 & 1.04 & 0.61 & 5.89 & 77.3 & 0.11 \\
\hline DV99-181 & Goerenger Well & $05 / 04 / 99$ & 7.89 & 61.2 & FA & 266 & 21.1 & 0.93 & 56.6 & 47.8 & 1.24 & 1.46 & 296 & 0.26 \\
\hline
\end{tabular}


Table 4: Continued

\begin{tabular}{|c|c|c|c|c|c|c|c|c|c|c|c|c|c|c|}
\hline Sample & Name or Description & Date & $\begin{array}{c}\text { pH } \\
\text { (lab) }\end{array}$ & $\mathrm{SiO}_{2}$ & $\begin{array}{c}\mathrm{SiO}_{2}{ }^{\mathrm{a}} \\
\text { Method }\end{array}$ & $\mathbf{N a}$ & $\mathbf{K}$ & Li & Ca & $\mathbf{M g}$ & $\mathrm{Sr}$ & $\mathbf{F}$ & $\mathrm{Cl}$ & $\mathrm{Br}$ \\
\hline \multicolumn{15}{|c|}{ Background Springs } \\
\hline DV97-46 & Sou Hot Spring & $11 / 03 / 97$ & 7.98 & 64.2 & FA & 166 & 29.3 & 0.75 & 107 & 21.0 & 12.5 & 4.95 & 79.0 & 0.07 \\
\hline DV97-47 & Sou Hot Spring & $11 / 03 / 97$ & 7.75 & 62.7 & FA & 162 & 28.2 & 0.72 & 112 & 20.9 & 12.4 & 4.72 & 77.1 & 0.09 \\
\hline DV97-48 & Hyder Hot Spring & $11 / 03 / 97$ & 7.96 & 60.3 & FA & 325 & 20.5 & 1.71 & 42.1 & 10.1 & 1.23 & 7.38 & 46.6 & 0.07 \\
\hline DV97-50 & Edward Creek Spring & $11 / 04 / 97$ & 7.83 & 87.5 & FA & 50.7 & 7.61 & 0.16 & 33.3 & 1.65 & 0.14 & 2.18 & 26.4 & 0.05 \\
\hline DV 97-51b & Old Man Spring & $11 / 04 / 97$ & 8.07 & 44.9 & FA & 228 & 7.78 & 0.08 & 32.4 & 1.38 & 0.27 & 0.74 & 130 & 0.33 \\
\hline DV97-52 & Horse Heaven Spring & $11 / 04 / 97$ & 7.91 & 44.1 & FA & 152 & 7.53 & 0.04 & 36.2 & 4.36 & 0.44 & 0.58 & 141 & 0.21 \\
\hline DV97-56 & Dead Travertine Spring & $11 / 05 / 97$ & 7.93 & 27.8 & FA & 325 & 48.5 & 2.03 & 201 & 79.4 & 2.62 & 2.34 & 527 & 0.64 \\
\hline DV97-60 & Fault Line Spring & $11 / 06 / 97$ & 8.01 & 41.5 & FA & 162 & 11.9 & 0.36 & 67 & 18.5 & 1.51 & 2.62 & 32.1 & $<0.02$ \\
\hline DV97-61 & Lower Ranch Hot Spring & $11 / 06 / 97$ & 8.07 & 40.7 & FA & 141 & 11.4 & 0.28 & 37.5 & 13.2 & 0.73 & 3.09 & 29.9 & $<0.02$ \\
\hline DV97-62 & McCoy Hot Spring & $11 / 06 / 97$ & 8.00 & 35.7 & FA & 185 & 9.05 & 0.16 & 78.6 & 30.0 & 2.35 & 1.47 & 228 & 0.31 \\
\hline DV97-63 & Kyle Spring & $11 / 06 / 97$ & 8.18 & 17.1 & FA & 84.7 & 2.32 & 0.02 & 90.5 & 41.4 & 1.20 & 0.59 & 189 & 0.29 \\
\hline DV97-64 & Dago Spring & $11 / 06 / 97$ & 8.06 & 38.3 & FA & 186 & 1.96 & 0.05 & 105 & 29.3 & 0.93 & 1.31 & 253 & 0.35 \\
\hline DV97-65 & Mustang Spring & $11 / 06 / 97$ & 8.13 & 29.5 & FA & 262 & 1.43 & 0.06 & 105 & 76.4 & 2.21 & 0.9 & 311 & 0.49 \\
\hline DV97-66 & Kitten Spring & $11 / 06 / 97$ & 7.64 & 43.7 & FA & 50.6 & 3.89 & $<0.01$ & 95.6 & 35.4 & 0.55 & 0.1 & 202 & 0.25 \\
\hline DV97-68 & Big Horn Spring & $11 / 07 / 97$ & 7.88 & 36.4 & FA & 427 & 4.52 & 0.06 & 87.2 & 47.4 & 1.80 & 0.74 & 707 & 0.92 \\
\hline DV97-69 & Dixie Hot Spring & $11 / 07 / 97$ & 8.00 & 105 & FA & 194 & 4.94 & 0.42 & 11 & 0.12 & 0.068 & 11.1 & 161 & 0.28 \\
\hline DV97-72 & Horse Creek Spring & $11 / 07 / 97$ & 7.38 & 26.8 & FA & 16 & 0.98 & $<0.01$ & 12.4 & 2.01 & 0.11 & 0.15 & 7.95 & $<0.02$ \\
\hline DV98-106 & Stu's Seep & $04 / 30 / 98$ & 8.00 & 18.9 & FA & 284 & 7.40 & 0.07 & 33.3 & 47.2 & 1.17 & 0.58 & 257 & 0.16 \\
\hline DV98-112 & Hyder Hot Spring & $04 / 30 / 98$ & 7.73 & 57.6 & FA & 357 & 20.5 & 1.59 & 42.8 & 10.1 & 1.22 & 7.84 & 45.4 & 0.07 \\
\hline DV98-113 & Lower Ranch Hot Spring & $05 / 04 / 98$ & 7.89 & 36.0 & FA & 157 & 11.4 & 0.28 & 38.6 & 13.3 & 0.71 & 3.26 & 27.8 & $<0.02$ \\
\hline DV98-114 & McCoy Hot Spring & $05 / 04 / 98$ & 7.83 & 32.3 & FA & 206 & 9.12 & 0.18 & 79.4 & 30.4 & 2.26 & 1.52 & 219 & 0.26 \\
\hline DV98-117 & Sou Hot Spring & $05 / 04 / 98$ & 7.56 & 58.6 & FA & 174 & 28.7 & $<0.01$ & 106 & 20.7 & 12.6 & 5.28 & 74.6 & 0.11 \\
\hline DV98-118 & Big Horn Spring & $05 / 04 / 98$ & 7.83 & 33.8 & FA & 802 & 6.83 & 0.08 & 131 & 69.5 & 2.73 & 0.36 & 1359 & 1.18 \\
\hline DV98-120 & Dixie Hot Spring & $05 / 05 / 98$ & 8.36 & 107 & FA & 211 & 4.91 & 0.42 & 10.7 & 0.22 & 0.086 & 12.6 & 162 & 0.23 \\
\hline DV98-128 & Jersey Hot Spring & $05 / 05 / 98$ & 7.41 & 134 & FA & 188 & 17.5 & 1.13 & 23.1 & 3.12 & 0.57 & 9.27 & 37.8 & 0.04 \\
\hline DV98-129 & Upper Jersey Seep & $05 / 06 / 98$ & 8.17 & 109 & FA & 261 & 26.4 & 1.50 & 23.7 & 2.84 & 0.52 & 10.5 & 48.6 & 0.07 \\
\hline DV98-131 & Spring in Spring Canyon & $05 / 06 / 98$ & 7.80 & 52.2 & FA & 380 & 11.4 & 0.12 & 88.6 & 59.6 & 1.31 & 0.94 & 418 & 0.54 \\
\hline DV98-132 & Wild Rose Spring & $05 / 07 / 98$ & 7.75 & 35.7 & FA & 176 & 5.96 & 0.04 & 97.0 & 30.4 & 0.92 & 1.01 & 135 & 0.13 \\
\hline DV98-169 & Lofthouse Spring & $05 / 07 / 98$ & 7.99 & 20.8 & FA & 40.5 & 1.36 & $<0.01$ & 58.7 & 15.6 & 0.67 & 0.30 & 43.2 & 0.07 \\
\hline DV98-170 & Not-So-OK Spring & $10 / 27 / 98$ & 8.17 & 18.7 & $\mathrm{FA}$ & 33.5 & 3.32 & $<0.01$ & 56.2 & 13.9 & 0.58 & 0.30 & 30.6 & $<0.02$ \\
\hline DV98-176 & War Canyon Spring & $10 / 27 / 98$ & 8.28 & 16.8 & FA & 32.5 & 0.61 & 0.05 & 42.1 & 1.61 & 0.62 & 0.17 & 30.1 & 0.04 \\
\hline DV98-177 & Pine Spring & $10 / 28 / 98$ & 8.04 & 30.8 & FA & 31.8 & 3.20 & $<0.01$ & 28.3 & 14.9 & 0.14 & 0.22 & 29.0 & 0.04 \\
\hline DV98-178 & Basalt Spring & $10 / 28 / 98$ & 7.95 & 31.0 & FA & 18.0 & 2.22 & $<0.01$ & 22.2 & 12.6 & 0.24 & 0.07 & 20.8 & 0.03 \\
\hline
\end{tabular}


Table 4: Continued

\begin{tabular}{|c|c|c|c|c|c|c|c|c|c|c|c|c|c|c|}
\hline Sample & Name or Description & Date & $\begin{array}{c}\text { pH } \\
(\mathbf{l a b})\end{array}$ & $\mathrm{SiO}_{2}$ & $\begin{array}{c}\mathrm{SiO}_{2}{ }^{\mathrm{t}} \\
\text { Method }\end{array}$ & $\mathbf{N a}$ & $\mathbf{K}$ & $\mathbf{L i}$ & $\mathbf{C a}$ & Mg & $\mathrm{Sr}$ & $\mathbf{F}$ & $\mathrm{Cl}$ & $\mathbf{B r}$ \\
\hline DV98-179 & Upper Cherry Spring & $10 / 28 / 98$ & 7.85 & 21.4 & FA & 24.4 & 1.59 & $<0.01$ & 23.9 & 5.44 & 0.25 & 0.07 & 22.1 & $<0.02$ \\
\hline DV99-209 & Dead Travertine Spring & $05 / 07 / 99$ & 7.72 & 27.2 & FA & 331 & 48.9 & 2.05 & 200 & 76 & 2.51 & 2.46 & 551 & 0.49 \\
\hline DV99-210 & Road seep, Dead Travertine & $05 / 08 / 99$ & 7.96 & 31.7 & FA & 370 & 56.4 & 2.28 & 158 & 90.4 & 2.92 & 2.72 & 630 & 0.51 \\
\hline DV99-211 & Upper Spg, Lower Ranch & $05 / 09 / 99$ & 8.07 & 38.3 & FA & 149 & 12.1 & 0.27 & 40.3 & 13.3 & 0.68 & 3.06 & 27.8 & 0.05 \\
\hline \multicolumn{15}{|c|}{ Background Wells } \\
\hline DV97-49 & Hole in the Wall \#2 Well & $11 / 04 / 97$ & 7.93 & 8.8 & FA & 77.7 & 7.61 & 0.03 & 30.4 & 5.62 & 0.33 & 0.49 & 69.6 & 0.14 \\
\hline DV97-57 & Bolivia Artesian Well & $11 / 05 / 97$ & 8.08 & 26.1 & FA & 146 & 4.02 & 0.16 & 99.2 & 66.8 & 2.97 & 0.30 & 289 & 0.43 \\
\hline DV97-70 & Flowingwell@AA Tank & $11 / 07 / 97$ & 7.89 & 61.8 & FA & 60.3 & 4.11 & 0.05 & 24.2 & 1.48 & 0.19 & 5.43 & 21.2 & 0.08 \\
\hline DV97-71 & Shaw Well & $11 / 07 / 97$ & 7.92 & 65.3 & FA & 68.3 & 3.15 & 0.06 & 14.8 & 0.74 & 0.11 & 7.21 & 19.7 & $<0.02$ \\
\hline DV98-115 & Irrigation Well & $05 / 04 / 98$ & 7.87 & 47.5 & $\mathrm{FA}$ & 221 & 12.6 & 0.53 & 62.3 & 35.1 & 1.44 & 1.87 & 72.4 & 0.10 \\
\hline DV98-116 & Brinkerhoff Well & $05 / 04 / 98$ & 7.78 & 36.0 & $\mathrm{FA}$ & 236 & 6.94 & 0.31 & 191 & 48.7 & 1.42 & 0.57 & 469 & 0.56 \\
\hline DV98-172 & Bernice Well & $10 / 27 / 98$ & 8.35 & 3.0 & $\mathrm{FA}$ & 94.2 & 2.13 & 0.02 & 31.7 & 55.3 & 0.31 & 0.14 & 80.7 & 0.13 \\
\hline \multicolumn{15}{|c|}{ Background Streams/Rain } \\
\hline DV97-58 & Cottonwood Creek & $11 / 05 / 97$ & 8.12 & 34.5 & FA & 147 & 3.17 & 0.04 & 66.2 & 46.3 & 0.97 & 0.31 & 216 & 0.32 \\
\hline DV98-107 & Unnamed Crk, Stu's Seep & $04 / 30 / 98$ & 8.42 & 21.1 & FA & 307 & 9.72 & 0.07 & 48.5 & 52.5 & 1.25 & 0.51 & 322 & 0.22 \\
\hline DV98-110 & Cottonwood Creek & $05 / 01 / 98$ & 8.03 & 38.3 & FA & 155 & 6.40 & 0.08 & 89.4 & 40.7 & 0.82 & 0.41 & 191 & 0.15 \\
\hline DV98-119 & Unnamed Stream & $05 / 05 / 98$ & 8.20 & 20.1 & FA & 262 & 3.40 & 0.04 & 61.6 & 49.1 & 0.90 & 0.43 & 306 & 0.24 \\
\hline DV98-121 & White Rock Canyon & $05 / 05 / 98$ & 8.44 & 16.8 & FA & 112 & 2.44 & 0.01 & 52.3 & 28.8 & 0.70 & 0.34 & 96.2 & 0.06 \\
\hline DV $98-125$ & Rain, Lizard Well Tank & $05 / 06 / 98$ & 7.03 & 7.8 & FA & 15.0 & 3.47 & 0.01 & 14.9 & 1.75 & 0.19 & 0.21 & 9.12 & $<0.02$ \\
\hline DV98-126 & Home Station Wash & $05 / 06 / 98$ & 7.47 & 33.0 & FA & 38.3 & 2.72 & 0.01 & 18.1 & 3.88 & 0.15 & 0.39 & 23.2 & $<0.02$ \\
\hline DV98-127 & Cedar Canyon Wash & $05 / 06 / 98$ & 7.52 & 35.3 & $\mathrm{FA}$ & 46.8 & 3.63 & 0.02 & 26.3 & 4.66 & 0.24 & 0.43 & 27.2 & $<0.02$ \\
\hline DV $98-130$ & Bucher Creek & $05 / 06 / 98$ & 7.90 & 31.2 & $\mathrm{FA}$ & 42.3 & 3.33 & 0.01 & 29.7 & 6.82 & 0.26 & 0.39 & 24.5 & $<0.02$ \\
\hline DV98-171 & Not-So-OK Creek & $10 / 27 / 98$ & 8.28 & 18.6 & $\mathrm{FA}$ & 34.0 & 1.53 & $<0.01$ & 52.7 & 12.6 & 0.54 & 0.24 & 30.1 & $<0.02$ \\
\hline DV98-173 & Bernice Creek & $10 / 27 / 98$ & 8.43 & 21.2 & FA & 129 & 3.65 & 0.05 & 62.4 & 78.2 & 0.83 & 0.32 & 128 & 0.16 \\
\hline DV98-174 & Hoyt Creek & $10 / 27 / 98$ & 8.72 & 17.1 & FA & 403 & 4.23 & 0.09 & 64.3 & 181 & 1.86 & 0.35 & 449 & 0.52 \\
\hline DV98-180 & Mt. Augusta Creek & $10 / 28 / 98$ & 7.72 & 19.0 & $\mathrm{FA}$ & 14.3 & 1.71 & $<0.01$ & 12.2 & 2.49 & 0.13 & 0.11 & 8.36 & $<0.02$ \\
\hline DV99-213 & Dixie Salt Lake & $05 / 10 / 99$ & 7.95 & 88.4 & $\mathrm{FA}$ & 4400 & 102 & 3.65 & 463 & 132 & 20.4 & 10.9 & 5310 & 5.51 \\
\hline \multicolumn{15}{|c|}{ Fumarole Condensates } \\
\hline DV $97-43$ & Crack 4 Fumarole & $11 / 03 / 97$ & -- & ... & -- & 1.12 & 0.08 & $<0.01$ & 0.39 & 0.05 & --- & $<0.01$ & 0.24 & $<0.04$ \\
\hline DV97-44 & Senator Fumarole & $11 / 03 / 97$ & --- & --- & --- & 4.3 & 1.24 & $<0.01$ & 4.01 & 0.79 & --- & 0.06 & 2.90 & $<0.04$ \\
\hline DV98-108 & Senator Fumarole & $05 / 01 / 98$ & 7.11 & 1.8 & $\mathrm{~T}$ & 0.23 & 0.06 & $<0.01$ & 0.04 & 0.06 & --- & $<0.01$ & 0.44 & $<0.02$ \\
\hline
\end{tabular}




\section{Table 4: Continued}

\begin{tabular}{|c|c|c|c|c|c|c|c|c|c|c|c|c|c|c|}
\hline Sample & Name or Description & Date & $\begin{array}{c}\text { pH } \\
(\text { lab) }\end{array}$ & $\mathrm{SiO}_{2}$ & $\begin{array}{c}\mathrm{SiO}_{2}{ }^{\mathrm{a}} \\
\text { Method }\end{array}$ & $\mathrm{Na}$ & $\mathbf{K}$ & $\mathbf{L i}$ & $\mathbf{C a}$ & $\mathbf{M g}$ & $\mathrm{Sr}$ & $\mathbf{F}$ & Cl & $\mathrm{Br}$ \\
\hline DV98-109 & Calcite Fumarole & $05 / 01 / 98$ & 6.56 & 1.6 & $\mathrm{~T}$ & 0.26 & 0.12 & $<0.01$ & 0.16 & 0.03 & --- & $<0.01$ & 0.18 & $<0.02$ \\
\hline DV98-164 & Senator Fumarole & $10 / 24 / 98$ & 6.72 & 5.5 & $\mathrm{~T}$ & 6.25 & $<0.01$ & $<0.01$ & 0.32 & 0.08 & --- & $<0.01$ & 9.41 & $<0.02$ \\
\hline DV98-165 & Calcite Fumarole & $10 / 25 / 98$ & 7.05 & 3.7 & $\mathrm{~T}$ & 0.17 & $<0.01$ & $<0.01$ & 0.25 & 0.01 & --- & $<0.01$ & 0.11 & $<0.02$ \\
\hline DV98-166 & South Bench Fumarole & $10 / 26 / 98$ & 3.13 & 2.5 & $\mathrm{~T}$ & 0.11 & $<0.01$ & $<0.01$ & 0.14 & 0.03 & --- & $<0.01$ & $<0.02$ & $<0.02$ \\
\hline
\end{tabular}

"Sample collection and analysis for silica varied; $\mathrm{FA}=$ filtered acidified, $\mathrm{FD}=$ filtered and diluted with deionized water, $\mathrm{T}=$ total (unacidified),

$\mathrm{TD}=$ total diluted with deionized water

bicarbonate and carbonate were analyzed in the field by $\mathrm{pH}$ titration on fresh samples.

"Bicarbonate and carbonate were analyzed in the labortory by $\mathrm{pH}$ titration on filtered, unacidified or raw, untreated samples. The values shown in bold type

were not corrected for excess silica. 


\section{Table 4: continued}

\section{Sample}

Brines

DIXE102-W

DV96-8

DV96-9

DV97-11

DV97-13

DV97-14

DV97-16

DV97-18

DV97-20

DV97-23

DV97-25

DV97-26

DV97-29

DV97-30

DV98-73

DV98-75

DV98-77

DV98-79

DV98-80

DV98-82

DV98-84

DV98-86

DV98-88

DV98-90

DV98-92

DV98-95

DV98-133

DV98-135

DV98-138

DV98-140

DV98-141

DV98-145

DV98-147

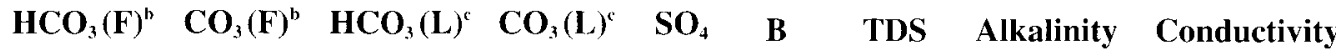 \\ (micromhos)}

$\begin{array}{ccccccccc}--- & --- & \mathbf{0} & \mathbf{7 6 . 3} & 225 & 9.35 & 2025 & --- & --- \\ --- & --- & \mathbf{3 2 . 8} & \mathbf{8 7 . 8} & 201 & 11.6 & 2026 & 173 & 2550 \\ 89.1 & 67.2 & \mathbf{1 8 . 2} & \mathbf{9 3 . 2} & 196 & 9.92 & 1855 & 170 & 2280 \\ 61 & 76.8 & 7.8 & 49.2 & 207 & 11.5 & 2078 & 161 & 2550 \\ 110 & 66 & 16.4 & 53.7 & 206 & 11.7 & 2042 & 172 & 2540 \\ 0 & 139 & 15 & 55.8 & 204 & 11.8 & 2076 & 183 & 2590 \\ 102 & 60 & 16.3 & 52.5 & 203 & 11.7 & 2072 & 168 & 2590 \\ 83 & 62.4 & 17.1 & 49 & 213 & 11.8 & 2084 & 162 & 2600 \\ 26.8 & 98.4 & 11.7 & 46.4 & 212 & 11.7 & 2028 & 164 & 2600 \\ 31.7 & 96 & 3.7 & 49.4 & 212 & 11.7 & 2040 & 159 & 2620 \\ 100 & 84 & 43.7 & 50.5 & 183 & 9.16 & 1907 & 188 & 2180 \\ 107 & 60 & 15.1 & 51.4 & 197 & 9.53 & 1930 & 173 & 2260 \\ 87.8 & 76.8 & 11 & 57.7 & 191 & 9.51 & 1918 & 172 & 2240 \\ 115 & 52.8 & 9.5 & 56.4 & 199 & 9.47 & 1938 & 169 & 2240 \\ 171 & 31.2 & 21.1 & 54.1 & 196 & 9.51 & 1334 & 177 & 2270 \\ 150 & 30.0 & 40.7 & 56.3 & 183 & 9.03 & 1282 & 188 & 2170 \\ 173 & 14.4 & 18.7 & 55.1 & 197 & 9.19 & 1303 & 174 & 2250 \\ 73.2 & 72.0 & 18.9 & 47.2 & 199 & 9.38 & 1329 & 171 & 2270 \\ 117 & 38.4 & 15.4 & 49.2 & 204 & 11.9 & 1494 & 169 & 2610 \\ 120 & 28.8 & 10.1 & 46.5 & 211 & 11.7 & 1502 & 156 & 2640 \\ 117 & 38.4 & 10.5 & 44.3 & 209 & 11.3 & 1494 & 163 & 2620 \\ 70.8 & 55.2 & 5.6 & 44.7 & 214 & 12.0 & 1505 & 153 & 2620 \\ 80.5 & 55.2 & 4.0 & 41.8 & 210 & 11.5 & 1473 & 150 & 2610 \\ 80.5 & 62.4 & 9.8 & 46.4 & 219 & 11.9 & 1509 & 156 & 2620 \\ 89.1 & 55.2 & 7.7 & 42.4 & 218 & 11.6 & 1510 & 153 & 2620 \\ 56.1 & 66.0 & 6.4 & 40.9 & 216 & 11.4 & 1496 & 153 & 2630 \\ --- & --- & 57.1 & 55.9 & 181 & 9.00 & 1713 & 190 & 2120 \\ --- & --- & 0 & 65.6 & 225 & 10.9 & 1947 & 224 & 2560 \\ --- & --- & 21.0 & 58.7 & 198 & 9.50 & 1777 & 180 & 2240 \\ 105 & 64.8 & 27.4 & 57.8 & 197 & 9.53 & 1749 & 181 & 2230 \\ 75.6 & 76.8 & 17.7 & 58.3 & 199 & 9.73 & 1770 & 178 & 2250 \\ 112 & 60.0 & 19.7 & 58.6 & 210 & 12.2 & 1923 & 173 & 2560 \\ 48.8 & 91.2 & 3.6 & 50.8 & 219 & 11.7 & 1953 & 150 & 2630\end{array}$




\section{Table 4: Continued}

\section{Sample}

\section{DV98-148}

DV98-150

DV98-152

DV98-154

DV98- 156

DV98-159

DV99-182

DV99-184

DV99-186

DV99-188

DV99-190

DV99-194

DV99-196

DV99-197

DV99-199

DV99-204

DV74782786-brine 2

DV76781986-brine 4

DV453382186-brine 6

DV73782886-brine 8

DV321882686-brine 10

DV651882686-brine 12

No number

\section{Condensates}

DIXE102-S

DV96-7

DV96-10

DV97-12

DV97-15

DV97-17

DV97-19

DV97-21

$\begin{array}{llllll}\mathrm{HCO}_{3}(\mathrm{~F})^{\mathrm{b}} & \mathrm{CO}_{3}(\mathrm{~F})^{\mathrm{b}} & \mathrm{HCO}_{3}(\mathrm{~L})^{\mathrm{c}} & \mathrm{CO}_{3}(\mathrm{~L})^{\mathrm{c}} & \mathrm{SO}_{4} & \mathrm{~B}\end{array}$

\begin{tabular}{ccc}
85.4 & 62.4 & 7.4 \\
90.3 & 57.6 & 10.4 \\
107 & 48.0 & 2.8 \\
78.1 & 57.6 & 6.6 \\
56.1 & 86.4 & 13.1 \\
26.8 & 106 & 8.6 \\
87.4 & 50 & 17.1 \\
57.3 & 69 & 8.2 \\
15 & 88 & 3.7 \\
28.1 & 87 & 0.8 \\
86.6 & 42 & 0 \\
76.9 & 45 & 3.7 \\
91.1 & 44 & 2.8 \\
40.7 & 70 & 0.6 \\
110 & 70 & 27.1 \\
85 & 74 & 19 \\
83 & 78 & 22.2 \\
--- & --- & 109 \\
--- & --- & 90 \\
--- & --- & 126 \\
--- & --- & 131 \\
--- & $-\ldots$ & 140 \\
--- & $\ldots-$ & 221 \\
--- & --- & 140 \\
\hline 1 & &
\end{tabular}

1953

1957

1940

1955

1950

1945

2027

2005

2018

2029

2049

2063

2057

2072

1871

1876

1889

1822

1779

1726

1729

1721

1766

846
Alkalinity Conductivity

\section{(micromhos)}

2610

2570

2600

2590

2600

2600

2550

2590

2590

2630

2660

2650

2660

2650

2230

2270

2230

2070

2040

1859

1953

2040

2180

1162

$\begin{array}{rcccccccc}--- & --- & \mathbf{3 4 . 9} & \mathbf{0} & 3.35 & 0.12 & --- & --- & --- \\ 54.9 & 0 & \mathbf{5 1} & \mathbf{0} & 1.26 & 0.16 & 78.2 & 41.8 & 114 \\ --- & --- & \mathbf{4 2} & \mathbf{0} & 1.96 & 0.13 & 64.1 & 34.4 & 97.7 \\ --- & --- & \mathbf{1 0 1} & \mathbf{0} & 88.5 & 4.9 & --- & 83 & -- \\ --- & --- & \mathbf{5 9 . 3} & \mathbf{0} & 2.74 & 0.28 & --- & 49 & --- \\ --- & --- & \mathbf{5 7 . 8} & \mathbf{0} & 5.19 & 0.16 & --- & 47 & -- \\ --- & --- & \mathbf{4 7} & \mathbf{0} & 0.87 & 0.12 & --- & 39 & --- \\ --- & --- & \mathbf{5 4 . 9} & \mathbf{0} & 8.89 & 0.54 & --- & 45 & ---\end{array}$


Table 4: Continued

Sample

DV97-22

DV97-24

DV97-27

DV97-28

DV97-31

DV98-74

DV98-76

DV98-78

DV98-8I

DV98-83

DV98-85

DV98-87

DV98-89

DV98-91

DV98-93

$\omega$ DV98-94

DV98-101

DV98-136

DV98-137

DV98-139

DV98-142

DV98-144

DV98-146

DV98-149

DV98-151

DV98-153

DV98-155

DV98-157

DV98-158

DV99- 183

DV99- 185

DV99-187

DV99-189

DV99-191
$\begin{array}{llllll}\mathrm{HCO}_{3}(\mathrm{~F})^{\mathrm{h}} & \mathrm{CO}_{3}(\mathrm{~F})^{\mathrm{b}} & \mathrm{HCO}_{3}(\mathrm{~L})^{\mathrm{c}} & \mathrm{CO}_{3}(\mathrm{~L})^{\mathrm{c}} & \mathrm{SO}_{4} & \mathrm{~B}\end{array}$

\begin{tabular}{|c|c|}
\hline --- & --- \\
\hline --- & --- \\
\hline --- & --- \\
\hline --- & --- \\
\hline --- & --- \\
\hline --- & $\cdots$ \\
\hline-- & -- \\
\hline-- & -. \\
\hline$\ldots$ & --- \\
\hline --- & --- \\
\hline- & --- \\
\hline --- & --- \\
\hline-- & -.. \\
\hline --- & --- \\
\hline --- & --- \\
\hline --- & --- \\
\hline-- & --- \\
\hline --- & --- \\
\hline$\cdots$ & --- \\
\hline --- & --- \\
\hline --- & --- \\
\hline --- & --- \\
\hline --. & --- \\
\hline --- & --- \\
\hline --- & $\cdots$ \\
\hline--- & --- \\
\hline --- & --- \\
\hline --- & --- \\
\hline--- & --- \\
\hline --- & --- \\
\hline--- & -- \\
\hline- & --- \\
\hline --- & --- \\
\hline & --- \\
\hline
\end{tabular}

\begin{tabular}{|c|}
\hline 121 \\
45.8 \\
48.3 \\
46.1 \\
54.3 \\
42.0 \\
46.1 \\
29.4 \\
47.2 \\
48.5 \\
51.9 \\
49.9 \\
142 \\
120 \\
45.0 \\
49.1 \\
43.4 \\
39.5 \\
37.6 \\
45.7 \\
49.6 \\
48.6 \\
47.9 \\
44.1 \\
55.1 \\
73.6 \\
114 \\
70.1 \\
45.5 \\
44 \\
60 \\
46.9 \\
44.6 \\
76.6 \\
\hline
\end{tabular}

TDS

Alkalinity

Conductivity

(micromhos)

$\begin{array}{ccccc}105 & 6.35 & --- & 99 & --- \\ 0.28 & 0.16 & --- & 38 & --- \\ 2.50 & 0.16 & --- & 40 & --- \\ 0.49 & 0.09 & --- & 38 & --- \\ 7.32 & 0.44 & --- & 45 & --- \\ 5.60 & 0.22 & 61.9 & 34.4 & 96.3 \\ 2.86 & 0.24 & 64.2 & 37.8 & 99.0 \\ 14.2 & 0.31 & 58.6 & 24.1 & 107 \\ 2.72 & 0.26 & 64.2 & 38.7 & 102 \\ 3.86 & 0.22 & 68.3 & 39.8 & 104 \\ 7.42 & 0.55 & 107 & 42.5 & 176 \\ 2.57 & 0.21 & 56.1 & 40.9 & 105 \\ 138 & 7.80 & 1096 & 116 & 1870 \\ 108 & 5.71 & 818 & 98.4 & 1380 \\ 2.92 & 0.22 & 64.3 & 36.9 & 101 \\ 2.51 & 0.17 & 68.6 & 40.2 & 103 \\ 2.54 & 0.16 & 60.4 & 35.6 & 93.1 \\ 2.58 & 0.05 & 58.1 & 32.4 & 97.9 \\ 2.66 & 0.08 & 41.6 & 30.8 & 90.2 \\ 1.02 & 0.03 & 61.4 & 37.5 & 94.0 \\ 1.25 & 0.07 & 69.5 & 40.7 & 104 \\ 1.48 & 0.10 & 65.3 & 39.8 & 102 \\ 3.41 & 0.10 & 65.5 & 39.3 & 102 \\ 7.33 & 0.09 & 67.8 & 36.1 & 107 \\ 6.20 & 0.42 & 123 & 45.2 & 176 \\ 42.3 & 2.21 & 424 & 60.3 & 608 \\ 112 & 5.81 & 1062 & 93.4 & 1426 \\ 38.0 & 2.03 & 393 & 57.5 & 545 \\ 2.34 & 0.14 & 62.9 & 37.3 & 97.0 \\ 2.55 & 0.13 & 65.8 & 36.1 & 96.6 \\ 24.9 & 1.39 & 280 & --- & --- \\ 2.45 & 0.15 & 69.8 & 38.4 & 94.4 \\ 2.96 & 0.10 & 67.7 & 36.6 & 97.3 \\ 51.3 & 2.96 & 532 & 62.8 & 711\end{array}$


Table 4: Continued

\begin{tabular}{|c|c|c|c|c|c|c|c|c|c|}
\hline Sample & $\mathrm{HCO}_{3}(\mathrm{~F})^{\mathrm{b}}$ & $\mathrm{CO}_{3}(\mathrm{~F})^{\mathrm{b}}$ & $\mathrm{HCO}_{3}(\mathrm{~L})^{\mathrm{c}}$ & $\mathrm{CO}_{3}(\mathrm{~L})^{\mathrm{c}}$ & $\mathrm{SO}_{4}$ & $\mathbf{B}$ & TDS & Alkalinity & $\begin{array}{l}\text { Conductivity } \\
\text { (micromhos) }\end{array}$ \\
\hline DV99-192 & --- & -- & 85.3 & 0 & 77.9 & 4.19 & 724 & 69.9 & 960 \\
\hline DV99-193 & --- & --- & 44.8 & 0 & 5.65 & 0.16 & 71.3 & 36.7 & 98.1 \\
\hline DV99-195 & --- & --- & 83.5 & 0 & 74.5 & 3.95 & 696 & 68.4 & 926 \\
\hline DV99-201 & --- & --- & 37.7 & 0 & 4.11 & 0.18 & 66.2 & 30.9 & 98.1 \\
\hline DV99-202 & --- & --- & 18.8 & $\mathbf{0}$ & 20.0 & 0.11 & 55.4 & 15.4 & 106 \\
\hline DV99-203 & --- & --- & 35 & $\mathbf{0}$ & 2.35 & 0.13 & 53.3 & 28.7 & 88.4 \\
\hline DV74782786-cond 1 & --- & -- & 53.6 & $\mathbf{0}$ & 4.7 & 0.043 & 80.0 & 43.9 & 108 \\
\hline DV76781986-cond 3 & -- & --- & 58.9 & 0 & 2.01 & 0.057 & 84.5 & 48.3 & 109 \\
\hline DV453382886-cond 5 & --- & --- & 20 & 0 & 28.1 & 0.057 & 69.5 & 16.4 & 118 \\
\hline DV73782886-cond 7 & --- & --- & 52.8 & $\mathbf{0}$ & 4.87 & 0.052 & 80.5 & 43.3 & 105 \\
\hline DV321882686-cond 9 & --- & --- & 63.3 & $\mathbf{0}$ & 9.52 & 0.071 & 99.8 & 51.9 & 136 \\
\hline DV651882686-cond 11 & --- & -- & 66.2 & $\mathbf{0}$ & 3.1 & 0.054 & 92.7 & 54.3 & 121 \\
\hline \multicolumn{10}{|c|}{ Injection Well/Power Plant Fluids } \\
\hline DV96-2 & 8.5 & 0 & 7.5 & $\mathbf{0}$ & 26.4 & 0.24 & 109 & 6.1 & 176 \\
\hline DV96-3 & 0 & 73.2 & 0 & 58 & 228 & 11.5 & 2078 & 186 & 2580 \\
\hline DV96-4 & 31.7 & 92.4 & 0 & 57.7 & 213 & 11.4 & 2005 & 175 & 2510 \\
\hline DV96-5 & 0 & 104 & 0 & 62.6 & 224 & 12.6 & 2067 & 190 & 2690 \\
\hline DV96-6 & 39 & 99.6 & $\mathbf{0}$ & 59.9 & 226 & 12.5 & 2119 & 192 & 2700 \\
\hline DV97-32 & --- & --- & 9.7 & 0 & 49.9 & 1.5 & -.- & 8 & \\
\hline DV97-33 & 0 & 91.2 & 0 & 19.6 & 217 & 12 & 2093 & 188 & 2690 \\
\hline DV97-34 & 0 & 84 & 0 & 18.3 & 196 & 10.7 & 1868 & 161 & 2370 \\
\hline DV97-35 & 19.5 & 88.8 & 0 & 18.7 & 198 & 10.6 & 1870 & 162 & 2380 \\
\hline DV97-36 & 68.3 & 84 & 0 & 20.1 & 222 & 12.1 & 2095 & 191 & 2720 \\
\hline DV97-37 & 0 & 122 & 0 & 20.5 & 222 & 12.0 & 2066 & 188 & 2700 \\
\hline DV97-40 & 0 & 98.4 & 0 & 19 & 219 & 11.9 & 2053 & 188 & 2700 \\
\hline DV97-4I & --- & --- & $<0.8$ & 0 & 51.4 & 1.51 & --- & 0 & --- \\
\hline DV97-42 & 37.8 & 98.4 & 18.3 & 55.5 & 193 & 9.43 & 1887 & 173 & 2220 \\
\hline DV98-97 & --- & --- & 16.2 & 0 & 21.5 & 0.36 & 90.4 & 13.3 & 168 \\
\hline DV98-98 & 17.1 & 91.2 & 0 & 14.5 & 228 & 11.9 & 1571 & 182 & 2750 \\
\hline DV98-143 & 0 & 103 & 0 & 22.0 & 229 & 12.6 & 2026 & 184 & 2720 \\
\hline DV98-161 & --- & --- & 16.4 & 0 & 47.8 & 0.96 & 211 & 13.4 & 340 \\
\hline DV98-162 & 0 & 81.6 & 0 & 21.7 & 230 & 12.2 & 2068 & 185 & 2720 \\
\hline DV98-163 & 222 & 0 & 202 & 0 & 117 & 1.88 & 734 & 166 & 1044 \\
\hline
\end{tabular}




\section{Table 4: Continued}

Sample

DV99-198

DV99-205

DV99-206

DV99-207

DV99-208

\section{$\begin{array}{llllll}\mathrm{HCO}_{3}(\mathrm{~F})^{\mathrm{h}} & \mathrm{CO}_{3}(\mathrm{~F})^{\mathrm{h}} & \mathrm{HCO}_{3}(\mathrm{~L})^{\mathrm{c}} & \mathrm{CO}_{3}(\mathrm{~L})^{\mathrm{c}} & \mathrm{SO}_{4} & \mathrm{~B}\end{array}$}

$\begin{array}{cc}212 & 0 \\ 0 & 84 \\ 0 & 52.8 \\ 45.1 & 0 \\ 0 & 73.2\end{array}$

Other Geothermal and On-Site Water Wells

DV96-1

DV97-38

DV97-39

DV97-53

DV97-54

DV97-55

DV97-59

DV97-67

$\omega$ DV98-96

DV98-99

DV98-100

DV98-102

DV98-103

DV98- 104

DV98-111

DV98-122

DV98- 123

Dixie Jack \#

Dixie Jack \#4

Dixie Jack \#7

DV98-160

DV98- 168

DV98- 175

DV99-181

\begin{tabular}{cc}
293 & 0 \\
298 & 0 \\
381 & 0 \\
--- & --- \\
--- & --- \\
--- & --- \\
--- & --- \\
--- & --- \\
366 & 0 \\
--- & -- \\
--- & --- \\
--- & --- \\
--- & --- \\
--- & --- \\
532 & 0 \\
--- & --- \\
--- & --- \\
--- & --- \\
--- & --- \\
--- & --- \\
361 & 0 \\
--- & --- \\
--- & --- \\
342 & 0 \\
\hline
\end{tabular}

TDS

14.8
17.1
17.2
0
18.1

\section{$124 \quad 1.93$}

$252 \quad 12.6$

$\begin{array}{lll}252 & 12.7 & 2173\end{array}$

$\begin{array}{lll}12.9 & 0.28 & 74.4\end{array}$

25

192
181
181
22
177

Conductivity

(micromhos)

1122

2740

2750

121

2730

$\begin{array}{ccccccc}\mathbf{3 0 5} & \mathbf{0} & 194 & 1.46 & 937 & -- & -.- \\ 297 & 0 & 191 & 1.65 & 921 & 249 & 1150 \\ 388 & 0 & 174 & 2.65 & 1190 & 318 & 1605 \\ \mathbf{5 3} & \mathbf{0} & 3.17 & 0.077 & 88.0 & 43.4 & 122 \\ \mathbf{5 9 . 6} & \mathbf{0} & 1.53 & 0.053 & 87.6 & 48.9 & 127 \\ \mathbf{5 9 . 8} & \mathbf{0} & 77.8 & 2.25 & 420 & 49 & 608 \\ \mathbf{2 2 6} & \mathbf{0} & 28.5 & 1.63 & 715 & 185 & 1039 \\ \mathbf{1 9 3} & \mathbf{0} & 84.7 & 5.79 & 3167 & 158 & 4850 \\ \mathbf{3 4 0} & \mathbf{2 0 . 3} & 177 & 2.71 & 1134 & 313 & 1705 \\ \mathbf{6 5 . 1} & \mathbf{0} & 54.2 & 2.03 & 329 & 53.4 & 557 \\ \mathbf{4 5 . 0} & \mathbf{0} & 2.91 & 0.14 & 63.8 & 36.9 & 99.3 \\ \mathbf{5 4 . 1} & \mathbf{0} & 16.9 & 0.51 & 159 & 44.3 & 282 \\ 89.5 & 0 & 195 & 6.77 & 1322 & 101 & 2250 \\ 162 & 0 & 80.2 & 5.78 & 2747 & 158 & 4890 \\ 688 & 0 & 219 & 5.52 & 1873 & 836 & 2150 \\ \mathbf{6 6 6} & \mathbf{0} & 161 & 6.66 & 1689 & 546 & 2300 \\ \mathbf{9 8 . 2} & \mathbf{6 . 9} & 334 & 1.26 & 910 & 92.0 & 1447 \\ \mathbf{4 3 0} & \mathbf{0} & 240 & 7.08 & 1527 & 352 & 2140 \\ \mathbf{1 3 1} & \mathbf{0} & 135 & 8.10 & 1020 & 107 & 1522 \\ \mathbf{3 9 . 7} & \mathbf{0} & 11.5 & 1.21 & 92.4 & 32.5 & 121 \\ \mathbf{3 3 8} & \mathbf{1 9 . 8} & 182 & 2.77 & 1207 & 310 & 1680 \\ \mathbf{2 4 3} & \mathbf{1 9 . 2} & 88.1 & 7.25 & 982 & 231 & 1227 \\ \mathbf{1 0 1 6} & \mathbf{0} & 219 & 5.48 & 2011 & 833 & 2080 \\ \mathbf{3 2 0} & \mathbf{2 0 . 2} & 204 & 2.81 & 1303 & 292 & 1728\end{array}$


Table 4: Continued

\section{Sample}

\section{Background Springs}

DV97-46

DV97-47

DV97-48

DV97-50

DV 97-5lb

DV97-52

DV97-56

DV97-60

DV97-61

DV97-62

DV97-63

DV97-64

DV97-65

V97-66

DV97-69

DV97-72

DV98-106

DV98-112

DV98-113

DV98-114

DV98-117

DV98-118

DV98- 120

DV98-128

DV98-129

DV98-131

DV98-132

DV98-169

DV98-170

DV98-176

DV98-177

DV98- 178
$\begin{array}{llllll}\mathrm{HCO}_{3}(\mathrm{~F})^{\mathrm{b}} & \mathrm{CO}_{3}(\mathrm{~F})^{\mathrm{b}} & \mathrm{HCO}_{3}(\mathrm{~L})^{\mathrm{c}} & \mathrm{CO}_{3}(\mathrm{~L})^{\mathrm{c}} & \mathrm{SO}_{4} & \mathrm{~B}\end{array}$

TDS

Alkalinity Conductivity

(micrombos)

$\begin{array}{ccccccccc}--- & --- & \mathbf{2 7 8} & \mathbf{1 6 . 5} & 385 & 1.46 & 1169 & 255 & 1423 \\ --- & --- & \mathbf{3 1 5} & \mathbf{0} & 379 & 1.39 & 1179 & 258 & 1412 \\ --- & --- & \mathbf{8 1 7} & \mathbf{3 1 . 3} & 119 & 4.24 & 1490 & 722 & 1598 \\ -- & --- & \mathbf{1 3 9} & \mathbf{0} & 58.7 & 0.31 & 409 & 114 & 434 \\ --- & --- & \mathbf{1 8 5} & \mathbf{9 . 9} & 228 & 0.65 & 870 & 168 & 1250 \\ --- & --- & \mathbf{1 9 3} & \mathbf{0} & 113 & 0.59 & 695 & 158 & 957 \\ --- & --- & \mathbf{4 1 9} & \mathbf{2 8 . 9} & 422 & 1.21 & 2088 & 392 & 3010 \\ --- & --- & \mathbf{4 9 6} & \mathbf{2 6} & 133 & 1.4 & 994 & 450 & 1125 \\ --- & --- & \mathbf{4 0 8} & \mathbf{2 0 . 7} & 68.4 & 1.05 & 777 & 369 & 879 \\ --- & -- & \mathbf{2 9 2} & \mathbf{1 5 . 4} & 199 & 0.83 & 1079 & 265 & 1491 \\ --- & --- & \mathbf{2 1 9} & \mathbf{1 4 . 6} & 142 & 0.22 & 804 & 204 & 1177 \\ --- & --- & \mathbf{2 9 2} & \mathbf{2 0 . 1} & 179 & 0.97 & 1108 & 273 & 1589 \\ --- & --- & \mathbf{3 9 4} & \mathbf{2 6 . 5} & 333 & 1.05 & 1544 & 367 & 2130 \\ --- & --- & \mathbf{1 3 2} & \mathbf{0} & 123 & 0.45 & 693 & 108 & 1021 \\ --- & --- & \mathbf{2 2 1} & \mathbf{0} & 181 & 0.84 & 1716 & 181 & 2850 \\ --- & --- & \mathbf{9 3 . 5} & \mathbf{0} & 139 & 0.96 & 723 & 76.6 & 1011 \\ --- & --- & \mathbf{6 8 . 8} & \mathbf{0} & 8.05 & 0.09 & 144 & 56.4 & 152 \\ --- & --- & \mathbf{3 2 6} & \mathbf{1 8 . 9} & 213 & 1.63 & 1196 & 299 & 1774 \\ --- & --- & \mathbf{6 6 6} & \mathbf{0} & 118 & 4.01 & 1508 & 735 & 1596 \\ --- & --- & \mathbf{4 5 3} & \mathbf{0} & 66.2 & 1.03 & 773 & 371 & 887 \\ -- & --- & \mathbf{3 2 6} & \mathbf{0} & 186 & 0.84 & 1061 & 267 & 1496 \\ --- & --- & 282 & \mathbf{0} & 363 & 1.31 & 1106 & 261 & 1427 \\ --- & --- & \mathbf{2 1 3} & \mathbf{0} & 243 & 1.08 & 2830 & 175 & 4920 \\ --- & --- & 57.2 & 8.9 & 121 & 0.95 & 608 & 76.4 & 1006 \\ --- & --- & 267 & 0 & 103 & 1.36 & 755 & 302 & 923 \\ --- & --- & \mathbf{4 7 1} & \mathbf{2 4 . 2} & 147 & 1.79 & 1019 & 426 & 1254 \\ --- & --- & \mathbf{4 3 2} & \mathbf{0} & 328 & 0.97 & 1722 & 354 & 2470 \\ --- & --- & \mathbf{3 7 6} & \mathbf{0} & 192 & 0.90 & 1016 & 308 & 1357 \\ --- & --- & \mathbf{2 1 0} & \mathbf{0} & 67.7 & 0.25 & 459 & 172 & 579 \\ --- & --- & \mathbf{2 0 3} & \mathbf{0} & 62.9 & 0.17 & 423 & 166 & 523 \\ --- & --- & \mathbf{1 3 1} & \mathbf{0} & 31.7 & 0.15 & 290 & 107 & 366 \\ --- & --- & \mathbf{1 6 4} & \mathbf{0} & 28.1 & 0.17 & 331 & 134 & 396 \\ --- & --- & \mathbf{1 1 7} & \mathbf{0} & 20.5 & 0.07 & 246 & 95.9 & 292 \\ & & & & & & & & \\ - & - & & & & & & & \end{array}$


Table 4: Continued

\begin{tabular}{|c|c|c|c|c|c|c|c|c|c|}
\hline Sample & $\mathrm{HCO}_{3}(\mathrm{~F})^{\mathrm{b}}$ & $\mathrm{CO}_{3}(\mathrm{~F})^{\mathrm{b}}$ & $\mathrm{HCO}_{3}(\mathrm{~L})^{\mathrm{c}}$ & $\mathrm{CO}_{3}(\mathrm{~L})^{\mathrm{c}}$ & $\mathrm{SO}_{4}$ & $\mathbf{B}$ & TDS & Alkalinity & $\begin{array}{l}\text { Conductivity } \\
\text { (micromhos) }\end{array}$ \\
\hline DV98-179 & --- & -- & 95.9 & $\mathbf{0}$ & 21.9 & 0.10 & 222 & 78.6 & 280 \\
\hline DV99-209 & --- & -- & 407 & 0 & 451 & 1.42 & 2101 & 376 & 2770 \\
\hline DV99-210 & --- & --- & 315 & 20.8 & 515 & 1.46 & 2199 & 310 & 3120 \\
\hline DV99-211 & --- & --- & 395 & 18.8 & 67.4 & 1.10 & 768 & 378 & 792 \\
\hline \multicolumn{10}{|c|}{ Background Wells } \\
\hline DV97-49 & --- & --- & 116 & 0 & 82.2 & 0.35 & 400 & 95.1 & 596 \\
\hline DV97-57 & --- & --- & 244 & 17 & 221 & 0.42 & 1118 & 228 & 1640 \\
\hline DV97-70 & --- & --- & 105 & 0 & 67.1 & 0.37 & 352 & 86.1 & 414 \\
\hline DV97-71 & --- & --- & 92 & $\mathbf{0}$ & 64.2 & 0.32 & 336 & 75.4 & 397 \\
\hline DV98-115 & --- & -- & 648 & 0 & 136 & 2.16 & 1201 & 531 & 1367 \\
\hline DV98-116 & --- & --- & 277 & $\mathbf{0}$ & 245 & 1.06 & 1514 & 227 & 2300 \\
\hline DV98-172 & -- & -- & 282 & 15.1 & 138 & 0.24 & 704 & 256 & 940 \\
\hline \multicolumn{10}{|c|}{ Background Streams/Rain } \\
\hline DV97-58 & --- & --- & 274 & 18 & 148 & 0.78 & 956 & 255 & 1374 \\
\hline DV98-107 & --- & -- & 257 & 22.9 & 222 & 1.95 & 1349 & 340 & 1975 \\
\hline DV98-110 & -- & --- & 264 & 17.3 & 153 & 0.74 & 920 & 245 & 1301 \\
\hline DV98-119 & --- & $\ldots$ & 219 & 15.4 & 241 & 1.12 & 1165 & 205 & 1795 \\
\hline DV98-121 & --- & --- & 264 & 21.3 & 82.7 & 0.55 & 661 & 252 & 886 \\
\hline DV98-125 & --- & --- & 61.3 & 0 & 16.4 & 0.23 & 124 & 50.2 & 173 \\
\hline DV98-126 & --- & --- & 97.6 & 0 & 22.6 & 0.19 & 208 & 80.0 & 284 \\
\hline DV98-127 & --- &.- & 99.4 & $\boldsymbol{\theta}$ & 62.0 & 0.19 & 272 & 81.5 & 384 \\
\hline DV98-130 & --- & -- & 144 & 0 & 32.0 & 0.27 & 284 & 118 & 371 \\
\hline DV98-171 & --- & -- & 171 & 7.1 & 62.9 & 0.15 & 391 & 152 & 495 \\
\hline DV98-173 & --- & --- & 365 & 23.6 & 223 & 0.32 & 1036 & 339 & 1348 \\
\hline DV98-174 & --- & --- & 409 & 44.5 & 756 & 0.84 & 2332 & 409 & 3180 \\
\hline DV98-180 & --- & --- & 61.1 & 0 & 8.71 & 0.06 & 129 & 50.1 & 149 \\
\hline DV99-213 & --- & --- & 314 & 54.6 & 3445 & 38.2 & 14390 & 415 & 20200 \\
\hline
\end{tabular}

Fumarole Condensates

DV97-43

DV97-44

DV98-108

$\begin{array}{rrrrrrrrr}--- & --- & -- & -- & 0.91 & 0.07 & --- & -- & -- \\ --- & --- & --- & --- & 37.8 & 0.11 & --- & -- & -- \\ -- & --- & \mathbf{7 5 . 4} & \mathbf{0} & 1.62 & 0.04 & 99.5 & 61.8 & 151\end{array}$


Table 4: Continued

Sample

DV98-109

DV98- 164

DV98- 165

DV98-166

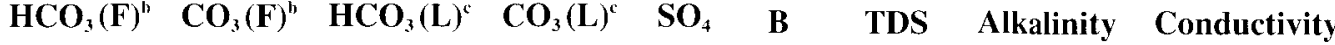

(micromhos)

$\begin{array}{ccccccc}--- & --- & \mathbf{1 9 5} & \mathbf{0} & 3.09 & <0.02 & 257 \\ -- & --- & \mathbf{6 2 . 2} & \mathbf{0} & 3.61 & <0.01 & 109 \\ -- & -- & \mathbf{1 8 8} & \mathbf{0} & 2.51 & <0.01 & 251 \\ -- & --- & \mathbf{0} & \mathbf{0} & 33.1 & <0.01 & 36 .\end{array}$

160
51.0
154
0

169

$--$ 
Table 5: Trace Element Chemistry for Various Geothermal and Regional Fluids, Dixie Valley Region, Nevada (values in ppm).

\begin{tabular}{|c|c|c|c|c|c|c|c|c|c|c|c|c|c|c|c|}
\hline $\begin{array}{l}\text { Sample } \\
\text { Brines }\end{array}$ & Name or Description & Date & Ag & Al (Total) & As & Au & Ba & $\mathrm{Be}$ & Cd & Co & $\mathrm{Cr}$ & Cs & $\mathrm{Cu}$ & $\mathrm{Fe}$ & $\mathbf{H g}$ \\
\hline DIXE $102-W$ & V102+V103 Separator & $10 / 02 / 95$ & --- & 1.41 & 0.46 & $\cdots$ & --- & --- & -- & --- & --- & 0.58 & --- & 0.02 & -- \\
\hline DV96-8 & 76-7 Well & $10 / 25 / 96$ & $<0.001$ & 1.12 & 0.51 & --- & 0.07 & 0.002 & $<0.002$ & $<0.002$ & $<0.002$ & 0.56 & 0.016 & 0.02 & $<0.0002$ \\
\hline DV96-9 & V101 Separator & $10 / 25 / 96$ & $<0.001$ & 1.54 & 0.47 & --- & 0.01 & 0.002 & $<0.002$ & $<0.002$ & $<0.002$ & 0.6 & 0.009 & $<0.01$ & $<0.0002$ \\
\hline DV97-11 & 73-7 Well & $10 / 29 / 97$ & $<0.001$ & 1.04 & 0.65 & $<0.002$ & 0.078 & 0.002 & $<0.00 \mathrm{l}$ & $<0.002$ & $<0.002$ & 0.56 & $<0.002$ & 0.04 & $<0.0001$ \\
\hline DV97-13 & 84-7 Well & $10 / 29 / 97$ & $<0.001$ & 1.12 & 0.52 & $<0.002$ & 0.077 & 0.002 & $<0.00$ & $<0.002$ & $<0.002$ & 0.56 & $<0,002$ & 0.02 & $<0.0001$ \\
\hline DV97-14 & 74-7 Well & $10 / 29 / 97$ & $<0.001$ & 1.13 & 0.65 & $<0.002$ & 0.073 & 0.002 & $<0.001$ & $<0.002$ & $<0.002$ & 0.6 & $<0.002$ & 0.01 & $<0.0001$ \\
\hline DV97-16 & V102+V103 Separator & $10 / 29 / 97$ & $<0.001$ & 1.12 & 0.65 & $<0.002$ & 0.074 & 0.002 & $<0.001$ & $<0.002$ & $<0.002$ & 0.53 & $<0.002$ & $<0.01$ & $<0.0001$ \\
\hline DV97-18 & V105 Separator & $10 / 29 / 97$ & $<0.001$ & 1.05 & 0.54 & $<0.002$ & 0.077 & 0.002 & $<0.001$ & $<0.002$ & $<0.002$ & 0.62 & 0.003 & 0.01 & $<0.0001$ \\
\hline DV97-20 & 82A-7 Well & $10 / 29 / 97$ & $<0.001$ & 1.02 & 0.49 & $<0.002$ & 0.078 & 0.002 & $<0.001$ & $<0.002$ & $<0.002$ & 0.59 & $<0.002$ & 0.02 & $<0.0001$ \\
\hline DV97-23 & 73B-7 Well & $10 / 30 / 97$ & $<0.001$ & 1.08 & 0.54 & $<0.002$ & 0.079 & 0.002 & $<0.001$ & $<0.002$ & $<0.002$ & 0.6 & 0.002 & $<0.01$ & $<0.0001$ \\
\hline DV97-25 & 27-33 Well & $10 / 30 / 97$ & $<0.001$ & 1.44 & 0.27 & $<0.002$ & 0.01 & 0.002 & $<0.001$ & $<0.002$ & $<0.002$ & 0.48 & $<0.002$ & $<0.01$ & $<0.0001$ \\
\hline DV97-26 & V101 Separator & $10 / 30 / 97$ & $<0.001$ & 1.47 & 0.37 & $<0.002$ & 0.011 & 0.002 & $<0.001$ & $<0.002$ & $<0.002$ & 0.5 & 0.004 & 0.01 & 0.0001 \\
\hline DV97-29 & 37-33 Well & $10 / 30 / 97$ & $<0.001$ & 0.99 & 0.19 & $<0.002$ & 0.007 & 0.002 & $<0.001$ & $<0.002$ & $<0.002$ & 0.53 & $<0.002$ & 0.02 & 0.107 \\
\hline DV97-30 & 28-33 Well & $10 / 30 / 97$ & $<0.001$ & 1.27 & 0.25 & $<0.002$ & 0.008 & 0.002 & $<0.001$ & $<0.002$ & $<0.002$ & 0.54 & $<0.002$ & 0.02 & 0.0001 \\
\hline DV98-73 & V101 Separator & $04 / 28 / 98$ & $<0.001$ & 1.34 & 0.49 & --- & 0.010 & ppm & $<0.001$ & $<0.002$ & $<0.002$ & 0.54 & $<0.002$ & $<0.01$ & 0.00012 \\
\hline DV98-75 & 27-33 Well & $04 / 28 / 98$ & $<0.001$ & 1.29 & 0.35 & --- & 0.010 & $-\cdots$ & $<0.001$ & $<0.002$ & $<0.002$ & 0.45 & $<0.002$ & 0.01 & 0.00007 \\
\hline DV98-77 & 37-33 Well & $04 / 28 / 98$ & $<0.001$ & 1.42 & 0.59 & $<0.0001$ & 0.010 & --- & $<0.001$ & $<0.002$ & $<0.002$ & 0.49 & $<0.002$ & $<0.01$ & 0.00061 \\
\hline DV98-79 & 28-33 Well & $04 / 28 / 98$ & $<0.001$ & 1.39 & 0.34 & --- & 0.010 & --- & $<0.001$ & $<0.002$ & $<0.002$ & 0.46 & $<0.002$ & $<0.01$ & $<0.00005$ \\
\hline DV98-80 & 76A-7 Well & $04 / 28 / 98$ & $<0.001$ & 1.04 & 0.73 & $<0.0001$ & 0.069 & 0.002 & $<0.001$ & $<0.002$ & $<0.002$ & 0.50 & $<0.002$ & $<0.01$ & 0.00034 \\
\hline DV98-82 & V102+ V103 Separator & $04 / 28 / 98$ & $<0.001$ & 1.08 & 0.82 & --- & 0.071 & --- & $<0.001$ & $<0.002$ & $<0.002$ & 0.54 & $<0.002$ & $<0.01$ & 0.00022 \\
\hline DV98-84 & 74-7 Well & $04 / 28 / 98$ & $<0.001$ & 1.09 & 0.74 & $<0.0001$ & 0.071 & --- & $<0.001$ & $<0.002$ & $<0.002$ & 0.59 & $<0.002$ & $<0.01$ & $<0.00005$ \\
\hline DV98-86 & 63-7 Well & $04 / 28 / 98$ & $<0.001$ & 1.03 & 0.72 & --- & 0.074 & --- & $<0.001$ & $<0.002$ & $<0.002$ & 0.61 & $<0.002$ & $<0.01$ & 0.00019 \\
\hline DV98-88 & 73-7 Well & $04 / 29 / 98$ & $<0.001$ & 1.04 & 0.70 & 0.0004 & 0.076 & --- & $<0.001$ & $<0.002$ & $<0.002$ & 0.56 & $<0.002$ & $<0.01$ & 0.00088 \\
\hline DV $98-90$ & 82A-7 Well & $04 / 29 / 98$ & $<0.00]$ & 1.01 & 0.72 & --- & 0.081 & --- & $<0.001$ & $<0.002$ & $<0.002$ & 0.61 & $<0.002$ & $<0.01$ & 0.00007 \\
\hline DV98-92 & V105 Separator & $04 / 29 / 98$ & $<0.001$ & 1.01 & 0.80 & --- & 0.080 & --- & $<0.001$ & $<0.002$ & $<0.002$ & 0.55 & $<0.002$ & $<0.01$ & $<0.00005$ \\
\hline DV98-95 & 73B-7 Well & $04 / 29 / 98$ & $<0.001$ & 0.97 & 0.69 & $<0.000 !$ & 0.085 & --- & $<0.001$ & $<0.002$ & $<0.002$ & 0.56 & $<0.002$ & $<0.01$ & 0.00028 \\
\hline DV98-133 & 27-33 Well & $10 / 20 / 98$ & $<0.001$ & 1.31 & 0.30 & --- & 0.012 & $<0.002$ & $<0.001$ & $<0.002$ & $<0.002$ & $0.5 !$ & $<0.002$ & 0.02 & 0.0003 \\
\hline DV98-135 & 27-33 Well & $10 / 20 / 98$ & $<0.001$ & 0.39 & 0.79 & $\cdots$ & 0.021 & 0.003 & $<0.001$ & $<0.002$ & $<0.002$ & 0.36 & $<0.002$ & $<0.01$ & 0.0010 \\
\hline DV98-138 & V101 Separator & $10 / 21 / 98$ & $<0.001$ & 1.32 & 0.35 & $\cdots$ & 0.012 & --. & $<0.001$ & $<0.002$ & $<0.002$ & 0.51 & $<0.002$ & $<0.01$ & 0.0013 \\
\hline DV98-140 & 37-33 Well & $10 / 21 / 98$ & $<0.001$ & 1.04 & 0.30 & --- & 0.005 & $--\cdot$ & $<0.001$ & $<0.002$ & $<0.002$ & 0.66 & $<0.002$ & $<0.01$ & 0.0021 \\
\hline DV98-141 & 28-33 Well & $10 / 21 / 98$ & $<0.001$ & 1.36 & 0.28 & --- & 0.010 & $<0.002$ & $<0.001$ & $<0.002$ & $<0.002$ & 0.48 & $<0.002$ & 0.02 & 0.0001 \\
\hline DV98-145 & 76A-7 Well & $10 / 22 / 98$ & $<0.001$ & 1.00 & 1.01 & --- & 0.077 & --- & $<0.001$ & $<0.002$ & $<0.002$ & 0.53 & $<0.002$ & 0.01 & 0.0001 \\
\hline DV98-147 & 63-7 Well & $10 / 22 / 98$ & $<0.001$ & 1.02 & 0.95 & --- & 0.069 & --- & $<0.001$ & $<0.002$ & $<0.002$ & 0.47 & $<0.002$ & $<0.01$ & $<0.0001$ \\
\hline DV98-148 & V102+V103 Separator & $10 / 22 / 98$ & $<0.001$ & 1.01 & 1.01 & $\cdots$ & 0.071 & 0.002 & $<0.001$ & $<0.002$ & $<0.002$ & 0.42 & $<0.002$ & $<0.01$ & 0.0002 \\
\hline DV98-150 & 74-7 Well & $10 / 22 / 98$ & $<0.001$ & 1.02 & 0.86 & $-\cdots$ & 0.071 & --- & $<0.001$ & $<0.002$ & $<0.002$ & 0.48 & $<0.002$ & $<0.01$ & $<0.0001$ \\
\hline DV98-152 & 73-7 Well & $10 / 22 / 98$ & $<0.001$ & 1.02 & 0.95 & $-\cdots$ & 0.079 & --- & $<0.001$ & $<0.002$ & $<0.002$ & 0.35 & $<0.002$ & $<0.01$ & 0.0004 \\
\hline DV98- 154 & 73B-7 Well & $10 / 22 / 98$ & $<0.001$ & 0.81 & 1.03 & --- & 0.071 & --- & $<0.001$ & $<0.002$ & $<0.002$ & 0.50 & $<0.002$ & $<0.01$ & 0.0001 \\
\hline DV98-156 & $82 \mathrm{~A}-7$ Well & $10 / 23 / 98$ & $<0.001$ & 0.90 & 0.81 & --- & 0.068 & --- & $<0.001$ & $<0.002$ & $<0.002$ & 0.48 & $<0.002$ & 0.01 & $<0.0001$ \\
\hline DV98-159 & V 105 Separator & $10 / 23 / 98$ & $<0.001$ & 0.99 & 0.80 & --- & 0.079 & --- & $<0.001$ & $<0.002$ & $<0.002$ & 0.43 & $<0.002$ & $<0.01$ & 0.0004 \\
\hline DV99-182 & 76A-7 Well & $05 / 04 / 99$ & $<0.001$ & 0.89 & 0.81 & --- & 0.074 & 0.002 & $<0.001$ & $<0.002$ & $<0.002$ & 0.93 & $<(0.002$ & $<0.01$ & $<(0.0001$ \\
\hline
\end{tabular}


Table 5: Continued

$\begin{array}{ll}\text { Sample } & \text { Name or Description } \\ \text { DV99-184 } & 74-7 \text { Well } \\ \text { DV99-186 } & \text { V102 + V103 Separator } \\ \text { DV99-188 } & 63-7 \text { Well } \\ \text { DV99-190 } & \text { 73-7 Well } \\ \text { DV99-194 } & \text { V105 Separator } \\ \text { DV99-196 } & \text { 82A-7 Well } \\ \text { DV99-197 } & \text { 73B-7 Well } \\ \text { DV99-199 } & 37-33 \text { Well } \\ \text { DV99-200 } & \text { 28-33 Well } \\ \text { DV99-204 } & \text { V101 Separator }\end{array}$

$\begin{array}{cccccccccccccc}\text { Date } & \text { Ag } & \text { Al (Total) } & \text { As } & \text { Au } & \text { Ba } & \text { Be } & \text { Cd } & \text { Co } & \text { Cr } & \text { Cs } & \text { Cu } & \text { Fe } & \text { Hg } \\ 05 / 04 / 99 & <0.001 & 0.97 & 0.73 & --- & 0.068 & 0.002 & <0.001 & <0.002 & <0.002 & 0.97 & <0.002 & <0.01 & <0.0001 \\ 05 / 04 / 99 & <0.001 & 0.97 & 0.8 & -- & 0.067 & <0.002 & <0.001 & <0.002 & <0.002 & 0.84 & <0.002 & <0.01 & <0.0001 \\ 05 / 04 / 99 & <0.001 & 0.98 & 0.82 & --- & 0.077 & 0.002 & <0.001 & <0.002 & <0.002 & 0.9 & <0.002 & <0.01 & <0.0001 \\ 05 / 04 / 99 & <0.001 & 1.0 & 0.83 & --- & 0.074 & 0.002 & <0.001 & <0.002 & <0.002 & 0.78 & <0.002 & <0.01 & <0.0001 \\ 05 / 05 / 99 & <0.001 & 0.95 & 0.7 & --- & 0.075 & 0.002 & <0.001 & <0.002 & <0.002 & 0.94 & <0.002 & <0.01 & 0.0001 \\ 05 / 05 / 99 & <0.001 & 0.86 & 0.78 & --- & 0.079 & 0.002 & <0.001 & <0.002 & <0.002 & 0.81 & <0.002 & <0.01 & <0.0001 \\ 05 / 05 / 99 & <0.001 & 0.96 & 0.75 & --- & 0.078 & 0.002 & <0.001 & <0.002 & <0.002 & 0.8 & <0.002 & <0.01 & <0.0001 \\ 05 / 05 / 99 & <0.001 & 1.37 & 0.48 & -- & 0.011 & 0.002 & <0.001 & <0.002 & <0.002 & 0.67 & <0.002 & <0.01 & <0.0001 \\ 05 / 05 / 99 & <0.001 & 1.37 & 0.49 & --- & 0.011 & 0.002 & <0.001 & <0.002 & <0.002 & 0.69 & <0.002 & <0.01 & 0.0001 \\ 05 / 05 / 99 & <0.001 & 1.3 & 0.43 & --- & 0.01 & 0.002 & <0.001 & <0.002 & <0.002 & 0.73 & <0.002 & <0.01 & 0.0001 \\ 08 / 27 / 86 & <0.001 & 1.1 & 0.81 & --- & 0.046 & 0.002 & <0.001 & <0.002 & <0.002 & 0.68 & <0.002 & 0.01 & <0.0001 \\ 08 / 19 / 86 & <0.001 & 1.19 & 0.86 & --- & 0.05 & 0.002 & <0.001 & <0.002 & <0.002 & 0.71 & <0.002 & <0.01 & 0.0003 \\ 08 / 21 / 86 & <0.001 & 1.52 & 0.68 & --- & 0.019 & 0.003 & <0.001 & <0.002 & <0.002 & 0.66 & <0.002 & 0.04 & 0.0003 \\ 08 / 28 / 86 & <0.001 & 0.99 & 0.79 & --- & 0.024 & 0.002 & <0.001 & <0.002 & <0.002 & 0.68 & <0.002 & 0.04 & 0.0006 \\ 08 / 26 / 86 & <0.001 & 0.75 & 0.75 & --- & 0.047 & 0.002 & <0.001 & <0.002 & <0.002 & 0.85 & <0.002 & 0.02 & 0.0005 \\ 08 / 26 / 86 & <0.001 & 0.69 & 0.95 & --- & 0.053 & 0.002 & <0.001 & <0.002 & <0.002 & 0.77 & <0.002 & 0.02 & <0.0001 \\ 09 / 23 / 93 & <0.001 & 0.88 & 0.2 & --- & 0.03 & <0.002 & <0.001 & <0.002 & <0.002 & 0.08 & 0.019 & 0.82 & 0.0009\end{array}$

DV74782786-brine 74-7 Well Archived DV7678 1986-brint 76-7 Well Archived DV453382186-brir 45-33 Well Archived DV73782886-brine 73-7 Well Archived DV321882686-brir 32-18 Well Archived DV651882686-brir 65-18 Well Archived

No number 28-33 Well Archived

$09 / 23 / 93$

\section{Condensates}

DIXE102-S

DV96-7

V $102+$ V 103 Separator

DV96-10

DV97-12

76-7 Well

DV97-15

DV97-17

DV97-19

DV $97-22$

DV97-24

DV $97-28$

DV97-3

DV98-74

DV98-76

DV98-78

DV98-81

DV98-83

DV98-85

DV98-87

DV98-89

V101 Separator

73-7 Well

74-7 Well

V $102+$ V 103 Separator

V105 Separator

82A-7 Well

73B-7 Well

V101 Separator

27-33 Wel

37-33 Well

28-33 Well

V10I Separator

27.33 Well

37-33 Wel

76A-7 Well

V102+V103 Separator

74.7 Well

63-7 Well

73-7 Well

DV98-9

82A-7 Well

10/02/95

$1025 / 96$

$10 / 25 / 96$

$10 / 29 / 97$

$10 / 29 / 97$

$10 / 29 / 97$

$10 / 29 / 97$

$10 / 29 / 97$

$10 / 29 / 97$

$10 / 30 / 97$

$10 / 30 / 97$

$10 / 30 / 97$

$10 / 30 / 97$

$04 / 28 / 98$

$04 / 28 / 98$

$04 / 28 / 98$

$04 / 28 / 98$

$04 / 28 / 98$

$<0.001<<0.01 \quad 0.0037$$$
\text { (1) }
$$$$
---
$$$$
--
$$$$
-
$$

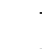$$
-
$$$$
---
$$$$
-
$$

$04 / 28 / 98$

$04 / 28 / 98$

$04 / 29 / 98$

$04 / 29 / 98$

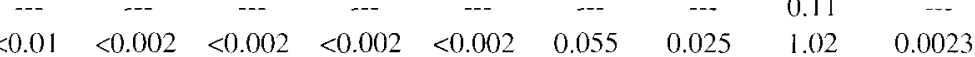

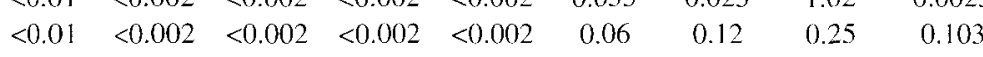

$$
\begin{aligned}
& \begin{array}{lllllllllll}
0.42 & -- & -- & -- & -- & -- & -- & -- & -- & \ldots & 0.039
\end{array}
\end{aligned}
$$

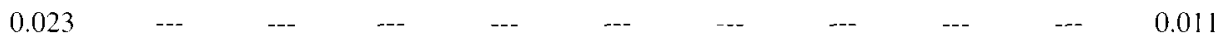

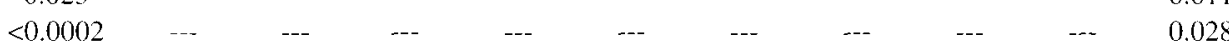

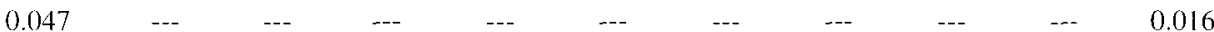

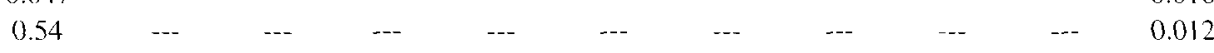

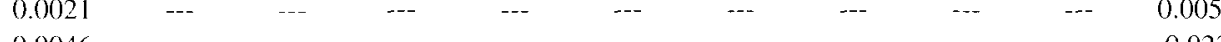

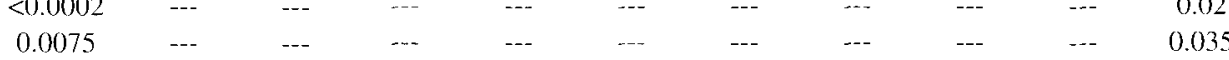

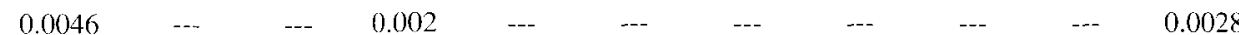

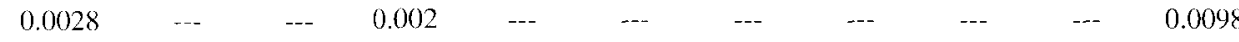

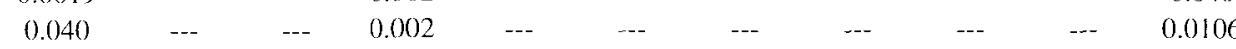

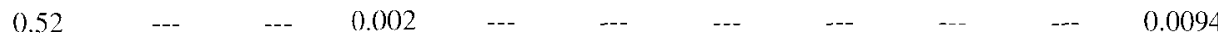

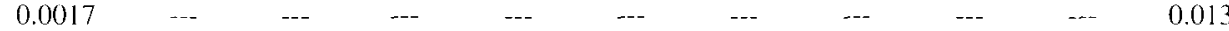

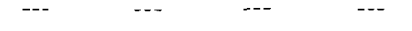

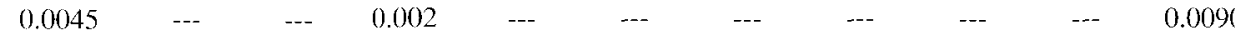

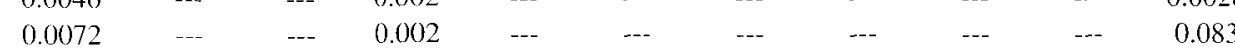

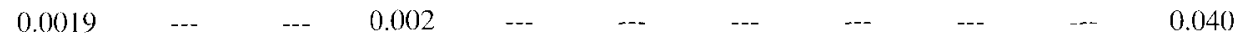

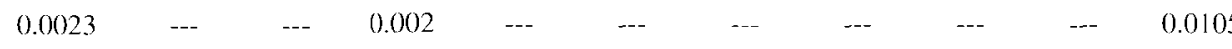


Table 5: Continued

\begin{tabular}{|c|c|c|c|c|c|c|c|c|c|c|c|c|c|c|c|}
\hline Sample & Name or Description & Date & Ag & Al (Total) & As & Au & $\mathbf{B a}$ & $\mathbf{B e}$ & Cd & Co & $\mathbf{C r}$ & $\mathrm{Cs}$ & $\mathrm{Cu}$ & $\mathbf{F e}$ & $\mathbf{H g}$ \\
\hline DV98-93 & V105 Separator & $04 / 29 / 98$ & --- & -- & 0.0036 & --- & --- & 0.002 & --- & --- & -- & --- & -- & --- & 0.015 \\
\hline DV98-94 & 73B-7 Well & $04 / 29 / 98$ & --- & --- & 0.0049 & $\cdots$ & --- & --- & --- & --- & --- & --- &.-- & --- & 0.0037 \\
\hline DV98-101 & $28-33$ Well & $04 / 30 / 98$ & --- & --- & 0.0024 & --- & --- & $\ldots$ & --- & --- & --- & --- &.-- & --- & 0.0039 \\
\hline DV98-136 & 27-33 Well & $10 / 20 / 98$ & --- & --- & 0.0020 & --- & $\ldots$ & $<0.002$ & --- & --- & --- & $\cdots$ & --- & --- & 0.0250 \\
\hline DV98-137 & V101 Separator & $10 / 21 / 98$ & --- & --- & 0.0009 & --- & --- & --- & --- & --- & $-\cdots$ & --- & --- & --- & 0.0156 \\
\hline DV98-139 & 37-33 Well & $10 / 21 / 98$ & --- & --- & 0.0011 & --- & --- & 0.002 & --- & --- & $\cdots$ & --- & --- & --- & 0.0024 \\
\hline DV98-142 & $28-33 \mathrm{Well}$ & $10 / 21 / 98$ & --- & --- & 0.0092 & $\cdots$ & --- & 0.002 & --- & --- & --- & --- & --- & --- & 0.0180 \\
\hline DV98-144 & 76A-7 Well & $10 / 22 / 98$ & --- & --- & 0.0010 & -- & --- & 0.002 & --- & --- & --- & --- & $\ldots$ & --- & 0.0162 \\
\hline DV98-146 & V102 + V103 Separator & $10 / 22 / 98$ & --- & --- & $<0.0002$ & --- & --- & 0.002 & --- & $-\cdots$ & -- & --- & --- & --- & 0.0218 \\
\hline DV98-149 & 63-7 Well & $10 / 22 / 98$ & --- & --- & 0.0004 & --- & --- & 0.002 & --- & --- & -- & --- & --- & --- & 0.0268 \\
\hline DV98-151 & 74-7 Well & $10 / 22 / 98$ & --- & --- & 0.027 &.-- & --- & 0.002 & --- & $\cdots$ & -- & --- & --- & --- & 0.0170 \\
\hline DV98-153 & 73-7 Well & $10 / 22 / 98$ & --- & --- & 0.22 & -- & --- & $<0.002$ & --- & --- & $-\cdots$ & --- & --- & --- & 0.0350 \\
\hline DV98-155 & 73B-7 Well & $10 / 22 / 98$ & --- &.-- & 0.57 & --- & --- & $<0.002$ & --- & --- & -- & --- & --- & --- & 0.0175 \\
\hline DV98-157 & $82 \mathrm{~A}-7$ Well & $10 / 23 / 98$ & --- & --- & 0.21 & --- & --- & $<0.002$ & --- & --- & $-\cdots$ & --- & --- & --- & 0.0368 \\
\hline DV98-158 & V105 Separator & $10 / 23 / 98$ & --- & --- & 0.0057 & --- & --- & --- & --- & --- & $\cdots$ & --- & --- & --- & 0.0223 \\
\hline DV99-183 & 76A-7 Well & $05 / 04 / 99$ & --- & $-\cdots$ & 0.0019 & --- & --- & --- & --- & --- & $-\cdots$ & --- & --- & --- & 0.0073 \\
\hline DV99-185 & 74-7 Well & $05 / 04 / 99$ & --- & $-\cdots$ & 0.1 & --- & --- & --- & --- & --- & -- & --- & $-\cdots$ & --- & 0.0092 \\
\hline DV99-187 & V102+ V103 Separator & $05 / 04 / 99$ & --- & --- & 0.002 & --- & --- & --- & --- & --- & -- & --- & --- & --- & 0.0056 \\
\hline DV99-189 & 63-7 Well & $05 / 04 / 99$ & --- & --- & 0.0023 & $\ldots$ & --- & --- & --- & --- & -- & --- & $-\ldots$ & --- & 0.0079 \\
\hline DV99-191 & 73-7 Well & $05 / 04 / 99$ & $\cdots$ & --- & 0.25 & $-\cdots$ & --- & --- & --- & --- & --- & --- & --- & --- & 0.0072 \\
\hline DV99-192 & 73B-7 Well & 05/04/99 & --- & --- & 0.4 & --- & --- & --- & --- & $\cdots$ & --- & -.- & --- & --- & 0.010 \\
\hline DV99-193 & V105 Separator & $05 / 05 / 99$ & --- & --- & $<0.0002$ & --- & --- & --- & --- & --- & --- & --- & --- & --- & 0.0024 \\
\hline DV99-195 & $82 \mathrm{~A}-7$ Well & $05 / 05 / 99$ & --- &.-- & 0.38 & --- & $\ldots$ & --- & --- & --- & --- & --- & --- & -- & 0.0101 \\
\hline DV99-201 & 28-33 Well & $05 / 05 / 99$ & --- & --- & 0.0024 & --- & --- & --- & --- & --- & -- & --- & --- & --- & 0.0031 \\
\hline DV99-202 & 37-33 Well & $05 / 05 / 99$ & --- & --- & $<0.0002$ & --- & --- & --- & $\cdots$ & --- & -.- & --- & --- & --- & 0.0047 \\
\hline DV99-203 & V101 Separator & $05 / 05 / 99$ & -.- & --- & $<0.0002$ & -- & --- & --- & --- & --- & --- & --- & --- & --- & 0.0077 \\
\hline DV74782786 & 74-7 Well Archived & $08 / 27 / 86$ & $\cdots$ & --- & $<0.0002$ & --- & --- & --- & -- & --- & $-\cdots$ & --- & --- & --- & 0.0004 \\
\hline DV76781986 & 76-7 Well Archived & $08 / 19 / 86$ &.-- & --- & $<0.0002$ & -- & --- & --- & $\cdots$ & --- & -- & --- & --- & --- & 0.001 \\
\hline DV45338288 & 45-33 Well Archived & $08 / 28 / 86$ & -.. & -- & $<0.0002$ & --- & --- & --- & $\ldots$ & --- & -- & --- & --- & $\cdots$ & 0.001 \\
\hline DV7.3782886 & d 73-7 Well Archived & $08 / 28 / 86$ & $\cdots$ & --- & 0.0006 & --- & --- & --- & --- & --- & --- & --- & --- & --- & 0.0005 \\
\hline DV 32188268 & 32-18 Well Archived & $08 / 26 / 86$ & --- & --- & $<0.0002$ & --- & --- & --- & -- & --- & $\cdots$ & --- & --- & $\cdots$ & 0.0016 \\
\hline DV65188268 & 65-18 Well Archived & $08 / 26 / 86$ & -- & --- & $<0.0002$ & -- & --- & --- & --- & --- & $\cdots$ & --- & --- & --- & 0.0015 \\
\hline \multicolumn{16}{|c|}{ Injection Well/Power Plant Fluids } \\
\hline DV96-2 & Condensate from plant & $10 / 24 / 96$ & $<0.001$ & 0.14 & 0.0024 & --- & $<0.01$ & $<0.002$ & $<0.002$ & $<0.002$ & $<0.002$ & $<0.002$ & 0.005 & 0.06 & $<0.0002$ \\
\hline DV96-3 & LP Brine @ Plant & $10 / 24 / 96$ & $<0.001$ & 1.27 & 0.74 & --- & 0.04 & 0.002 & $<0.002$ & $<0.002$ & 0.013 & 0.53 & $<0.002$ & 0.05 & $<0.0002$ \\
\hline DV96-4 & 45-5 Injection Well & $10 / 24 / 96$ & $<0.001$ & 1.21 & 0.86 & --- & 0.05 & 0.002 & $<0.002$ & $<0.002$ & $<0.002$ & 0.52 & $<0.002$ & 0.08 & $<0.0002$ \\
\hline DV96-5 & Lamb 1 Injection Well & $10 / 24 / 96$ & $<0.001$ & 1.39 & 0.82 & --- & 0.06 & 0.002 & $<0.002$ & $<0.002$ & $<0.002$ & 0.61 & $<0.002$ & $<0.01$ & $<0.0002$ \\
\hline DV96-6 & 65-18 Injection Well & $10 / 24 / 96$ & $<0.001$ & 1.35 & 1.44 & --- & 0.06 & 0.002 & $<0.002$ & $<0.002$ & $<0.002$ & 0.59 & $<0.002$ & 0.02 & $<0.0002$ \\
\hline DV $97-32$ & Condensate from plant & $10 / 31 / 97$ & --- & --- & 0.098 & --- & --- & --- & --- & --- & --- & --- & -.- & --- & 0.0004 \\
\hline DV97-33 & LP Brine & $10 / 31 / 97$ & $<0.001$ & 1.36 & 0.93 & $<0.002$ & 0.056 & 0.002 & $<0.001$ & $<0.002$ & 0.003 & 0.86 & 0.013 & $<0.01$ & 0.0002 \\
\hline
\end{tabular}


Table 5: Continued

$\begin{array}{ll}\text { Sample } & \text { Name or Description } \\ \text { DV97-34 } & \text { 25-5 + 45-5 Injectate } \\ \text { DV97-35 } & \text { 25-5 +45-5 Injectate } \\ \text { DV97-36 } & \text { 65-18 Injection Well } \\ \text { DV97-37 } & \text { 32-18 Injection Well } \\ \text { DV97-40 } & \text { LP Brine @ Plant } \\ \text { DV97-41 } & \text { Condensate from plant } \\ \text { DV97-42 } & \text { High P Brine @ plant } \\ \text { DV98-97 } & \text { Condensate from plant } \\ \text { DV98-98 } & \text { LP Brine @ Plant } \\ \text { DV98-143 } & \text { 25-5 Injection Well } \\ \text { DV98-161 } & \text { Condensate from plant } \\ \text { DV98-162 } & \text { LP Brine @ Plant } \\ \text { DV98-163 } & \text { 65-18 Injection Well } \\ \text { DV99-198 } & \text { 65-18 Injection Well } \\ \text { DV99-205 } & \text { 25-5 +45-5 Injectate } \\ \text { DV99-206 } & \text { LP Brine @ Plant } \\ \text { DV99-207 } & \text { Condensate from plant } \\ \text { DV99-208 } & \text { 52-18+41-18 Injectate }\end{array}$

$\begin{array}{cccc}\text { Date } & \text { Ag } & \text { Al (Total) } & \text { As } \\ 10 / 31 / 97 & <0.001 & 1.14 & 0.79 \\ 10 / 31 / 97 & <0.001 & 1.14 & 0.93 \\ 10 / 31 / 97 & <0.001 & 1.32 & 1.03 \\ 10 / 31 / 97 & <0.001 & 1.34 & 1.03 \\ 10 / 31 / 97 & <0.001 & 1.36 & 1.03 \\ 10 / 31 / 97 & --- & --- & 0.098 \\ 10 / 31 / 97 & <0.001 & 1.38 & 1.38 \\ 04 / 29 / 98 & --- & --- & 0.0043 \\ 04 / 29 / 98 & <0.001 & 1.22 & 0.92 \\ 10 / 21 / 98 & <0.001 & 1.19 & 1.06 \\ 10 / 23 / 98 & <0.001 & 0.07 & 0.060 \\ 10 / 23 / 98 & <0.001 & 1.22 & 1.02 \\ 10 / 23 / 98 & <0.001 & <0.02 & 0.034 \\ 05 / 05 / 99 & <0.001 & <0.02 & 0.0095 \\ 05 / 06 / 99 & <0.001 & 1.19 & 0.97 \\ 05 / 06 / 99 & <0.001 & 1.26 & 0.9 \\ 05 / 06 / 99 & <0.001 & 0.04 & 0.0041 \\ 05 / 06 / 99 & <0.001 & 1.32 & 0.98\end{array}$

$\begin{array}{cccccccccc}\text { Au } & \mathbf{B a} & \mathbf{B e} & \mathbf{C d} & \mathbf{C o} & \mathbf{C r} & \mathbf{C s} & \mathbf{C u} & \mathbf{F e} & \mathbf{H g} \\ <0.002 & 0.047 & 0.002 & <0.001 & <0.002 & <0.002 & 0.68 & <0.002 & 0.02 & <0.0001 \\ -- & 0.048 & 0.002 & <0.001 & <0.002 & <0.002 & 0.71 & <0.002 & 0.02 & <0.0001 \\ <0.002 & 0.052 & 0.002 & <0.001 & <0.002 & 0.003 & 0.8 & <0.002 & 0.01 & 0.0003 \\ <0.002 & 0.059 & 0.002 & <0.001 & <0.002 & 0.007 & 0.91 & <0.002 & 0.02 & <0.0001 \\ <0.002 & 0.058 & 0.002 & <0.001 & <0.002 & <0.002 & 0.75 & <0.002 & 0.02 & <0.0001 \\ --- & --- & --- & --- & --- & --- & --- & --- & --- & 0.0001 \\ <0.002 & 0.01 & 0.002 & <0.001 & <0.002 & <0.002 & 0.74 & 0.002 & 0.05 & 0.0001 \\ --- & --- & <0.002 & --- & --- & --- & --- & --- & --- & 0.00008 \\ --- & 0.059 & --- & <0.001 & <0.002 & <0.002 & 0.59 & <0.002 & <0.01 & 0.00022 \\ --- & 0.060 & --- & <0.001 & <0.002 & <0.002 & 0.54 & <0.002 & <0.01 & 0.0001 \\ --- & 0.006 & <0.002 & <0.001 & <0.002 & <0.002 & 0.016 & <0.002 & 0.07 & 0.0003 \\ --- & 0.063 & <0.002 & <0.001 & <0.002 & <0.002 & 0.51 & <0.002 & <0.01 & 0.0003 \\ --- & 0.026 & 0.002 & <0.001 & <0.002 & <0.002 & 0.005 & <0.002 & 0.43 & 0.0002 \\ --- & 0.024 & <0.002 & <0.001 & <0.002 & <0.002 & 0.041 & <0.002 & 0.04 & 0.0001 \\ --- & 0.055 & 0.002 & <0.001 & <0.002 & <0.002 & 0.83 & <0.002 & <0.01 & <0.0001 \\ --- & 0.059 & 0.002 & <0.001 & <0.002 & <0.002 & 0.86 & <0.002 & <0.01 & 0.0001 \\ --- & <0.002 & <0.002 & <0.001 & <0.002 & <0.002 & 0.011 & <0.002 & 0.08 & <0.0001 \\ --- & 0.058 & 0.002 & <0.001 & <0.002 & <0.002 & 0.87 & <0.002 & <0.01 & 0.0001\end{array}$

$\$$ Other Geothermal and On-Site Water Wells

DV96-1

Domestic Well

DV97-38

Domestic Well

DV97-39

Goerenger Well

DV97-53

46-32 Well

DV97-54 27-32 Well

DV97-55

DV97-59

27-32 Well

DV97-67

-W-5 Well

DV98-96

DV98-99

66-2l Wel

DV98-100

Goerenger Well

DV98-102

27-32 Well

DV98-103

DV98-104

DV98-122

DV98- 123

Dixie Jack \# 1

Dixie Jack \#4

Dixie Jack \#

45-14 Wel

45-14 Well

66-2I Well

62-21 Wel

97-2 Well

32-6 Well

Gradient Well DJ \#

Gradient Well DJ \#4

DV98-160

Goerenger Well

$\begin{array}{lccccccccccccc}10 / 24 / 96 & <0.001 & 0.2 & 0.052 & & 0.04 & <0.002 & <0.002 & <0.002 & <0.002 & 0.084 & 0.008 & 1.18 & <0.0002 \\ 10 / 31 / 97 & <0.001 & 0.1 & 0.053 & <0.002 & 0.044 & <0.002 & <0.001 & <0.002 & <0.002 & 0.12 & <0.002 & 0.88 & 0.0002 \\ 10 / 31 / 97 & <0.001 & 0.18 & 0.013 & <0.002 & 0.038 & <0.002 & <0.001 & <0.002 & <0.002 & 0.063 & 0.004 & 0.7 & 0.0016 \\ 11 / 05 / 97 & <0.001 & 0.03 & 0.008 & <0.002 & 0.005 & <0.002 & <0.0002 & <0.002 & <0.002 & 0.15 & <0.002 & 0.17 & 0.125 \\ 11 / 05 / 97 & <0.001 & 0.04 & 0.007 & <0.002 & <0.002 & <0.002 & 0.0002 & <0.002 & 0.002 & 0.035 & 0.006 & 0.2 & 0.159 \\ 11 / 05 / 97 & <0.001 & 0.13 & 0.31 & <0.002 & 0.016 & <0.002 & 0.0002 & <0.002 & <0.002 & 0.15 & 0.008 & 0.14 & 0.013 \\ 11 / 05 / 97 & <0.001 & 0.03 & 0.002 & --- & 0.014 & <0.002 & <0.0002 & <0.002 & <0.002 & 0.034 & <0.002 & 0.08 & 0.0003 \\ 11 / 07 / 97 & <0.001 & 0.05 & 0.23 & <0.002 & 0.33 & <0.002 & <0.0002 & <0.002 & <0.002 & 0.6 & <0.002 & 6.33 & 0.0004 \\ 04 / 29 / 98 & <0.001 & <0.02 & 0.015 & --- & 0.041 & <0.002 & <0.001 & <0.002 & <0.002 & 0.040 & <0.002 & 0.07 & 0.00025 \\ 04 / 29 / 98 & --- & --- & 0.34 & <0.0001 & --- & 0.002 & --- & --- & --- & -- & --- & --- & 0.085 \\ 04 / 29 / 98 & --- & --- & 0.0029 & <0.0001 & --- & --- & -- & --- & -- & --- & --- & --- & 0.038 \\ 04 / 30 / 98 & --- & --- & 0.10 & --- & --- & --- & -\ldots- & -- & --- & --- & --- & --- & 0.076 \\ 04 / 30 / 98 & <0.001 & 0.23 & 0.32 & --- & 0.055 & --- & <0.001 & <0.002 & <0.002 & 0.32 & <0.002 & 0.07 & 0.00053 \\ 04 / 30 / 98 & <0.001 & <0.02 & 0.14 & --- & 0.34 & <0.002 & <0.001 & <0.002 & <0.002 & 0.52 & <0.002 & 5.85 & 0.00012 \\ 05 / 01 / 98 & <0.001 & 0.09 & 0.56 & <0.0001 & 0.12 & <0.002 & <0.001 & <0.002 & <0.002 & 0.11 & <0.002 & 0.24 & 0.00090 \\ 05 / 05 / 98 & <0.001 & <0.02 & 0.023 & --- & 0.065 & <0.002 & <0.001 & <0.002 & 0.003 & <0.002 & 0.002 & 0.31 & 0.00031 \\ 05 / 06 / 98 & <0.001 & 0.04 & 0.0024 & --- & 0.014 & <0.002 & <0.001 & <0.002 & <0.002 & <0.002 & <0.002 & <0.01 & 0.00014 \\ 05 / 17 / 98 & <0.001 & <0.02 & 0.043 & <0.001 & 0.049 & <0.002 & <0.001 & <0.002 & <0.002 & 0.09 & <0.002 & 0.03 & 0.00046 \\ 05 / 20 / 98 & <0.001 & 0.07 & 0.074 & <0.001 & 0.043 & <0.002 & <0.001 & <0.002 & 0.002 & 0.28 & 0.002 & 0.08 & 0.00021 \\ 05 / 14 / 98 & <0.001 & 0.03 & 0.003 & <0.001 & 0.003 & <0.002 & <0.001 & <0.002 & <0.002 & 0.009 & <0.002 & 0.02 & 0.00021 \\ 10 / 23 / 98 & <0.001 & <0.02 & 0.014 & --- & 0.039 & 0.002 & <0.001 & <0.002 & <0.002 & 0.022 & <0.002 & 0.07 & 0.0001\end{array}$


Table 5: Continued

Sample

DV98-168

DV98- 175

DV99-181

Background Sprin

DV97-46

DV97-47

DV97-48

DV97-50

DV $97-51 \mathrm{~b}$

DV97-52

DV97-56

DV97-60

DV97-61

DV97-62

DV97-63

DV97-64

DV97-65

DV97-66

$\Perp \quad$ DV97-68

DV97-69

DV97-72

DV98-106

DV98-112

DV98-113

DV98-114

DV98-117

DV98-118

DV98-120

DV98- 128

DV98-129

DV98-131

DV98-132

DV98-169

DV98-170

DV98-176

DV98-177

DV98-178

DV98-179

DV99-209

DV99.210

DV99-211
Name or Description

38-32 Well

Goerenger Well

Sou Hot Spring

Sou Hot Spring

Hyder Hot Spring

Edward Creek Spring

Old Man Spring

Horse Heaven Spring

Dead Travertine Spring

Fault Line Spring

Lower Ranch Hot Spring

McCoy Hot Spring

Kyle Spring

Dago Spring

Mustang Spring

Kitten Spring

Big Horn Spring

Dixie Hot Spring

Horse Creek Spring

Stu's Seep

Hyder Hot Spring

Lower Ranch Hot Spring

McCoy Hot Spring

Sou Hot Spring

Big Horn Sprin

Dixie Hot Spring

Jersey Hot Spring

Upper Jersey Seep

Spring in Spring Canyon

Wild Rose Spring

Lofthouse Spring

Not-So-OK Spring

War Canyon Spring

Pine Spring

Basalt Spring

Upper Cherry Spring

Dead Travertine Spring

Dead Travertine, Road Seep

Upper Spring, Lower Ranch $\quad 05 / 09 / 99$
10/26/98

$10 / 28 / 98$

$05 / 04 / 90$

$11 / 03 / 97$

$11 / 03 / 97$

$11 / 03 / 97$

$11 / 04 / 97$

$11 / 04 / 97$

$11 / 04 / 97$

$11 / 05 / 97$

$11 / 06 / 97$

$11 / 06 / 97$

$11 / 06 / 97$

$11 / 06 / 97$

$11 / 06 / 97$

$11 / 06 / 97$

$11 / 06 / 97$

$11 / 07 / 97$

$11 / 07 / 97$

$11 / 07 / 97$

$04 / 30 / 98$

$04 / 30 / 98$

$05 / 04 / 98$

$05 / 04 / 98$

$05 / 04 / 98$

$05 / 04 / 98$

$05 / 05 / 98$

$05 / 05 / 98$

$05 / 06 / 98$

$05 / 06 / 98$

05/07/98

$05 / 07 / 98$

$10 / 27 / 98$

$10 / 27 / 98$

$10 / 28 / 98$

$10 / 28 / 98$

$10 / 28 / 98$

$5 / 07 / 99$
Ag Al (Total) As

$<0.001<0.02 \quad 0.13$

$<0.001<0.02 \quad 0.30$

$<0.001<0.02 \quad 0.012$

Au

$-$

Ba
0.062
0.13

Be

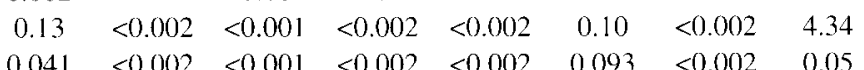

0.05

Hg

0.0010

0.0001

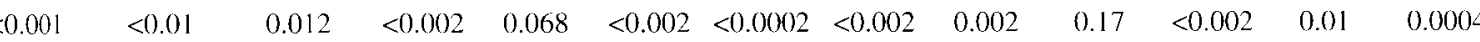
$\begin{array}{lllllllllllll}<0.001 & <0.01 & 0.012 & <0.002 & 0.066 & <0.002 & 0.0003 & <0.002 & <0.002 & 0.12 & 0.022 & 0.27 & 0.0001\end{array}$ $\begin{array}{lllllllllllll}<0.001 & 0.02 & 0.033 & <0.002 & 0.15 & <0.002 & 0.0011 & <0.002 & <0.002 & 0.35 & <0.002 & 0.04 & 0.0005\end{array}$ $<0.001<0.02<0.023<0.004<0.002<0.0002<0.002 \quad 0.005<0.002<0.002 \quad 0.03<<0.0001$ $\begin{array}{lllllllllllll}<0.001 & 0.02 & 0.017 & <0.002 & 0.02 & <0.002 & <0.0002 & <0.002 & <0.002 & <0.002 & <0.002 & 0.02 & <0.0001\end{array}$ $\begin{array}{lllllllllllll}<0.001 & <0.01 & 0.018 & -- & 0.035 & <0.002 & <0.0002 & <0.002 & <0.002 & <0.002 & <0.002 & 0.04 & 0.0003\end{array}$ $\begin{array}{lllllllllll}<001<0.01<0.01<18 & <0.002 & 0.028 & <0.002<0.0002<0.002 & 0.009 & 0.18 & 0.002 & <0.01 & 0.0002\end{array}$ $\begin{array}{lllllllllllll}<0.001 & <0.01 & 0.005 & <0.002 & 0.053 & <0.002 & <0.0002 & <0.002 & <0.002 & 0.047 & 0.002 & <0.01 & <0.0001\end{array}$ $\begin{array}{lllllllllllll}<0.001 & <0.01 & 0.008 & <0.002 & 0.11 & <0.002 & <0.0002 & <0.002 & <0.002 & 0.046 & 0.003 & <0.01 & 0.0002\end{array}$ $\begin{array}{lllllllllllll}<0.01 & 0.011<0.002 & 0.072<0.002 & <0.0002<0.002 & <0.002 & 0.014 & <0.002<0.01 & 0.0003\end{array}$ $\begin{array}{llllllllllllll}<0.001 & 0.01 & 0.003 & -- & 0.049 & <0.002 & <0.0002 & <0.002 & <0.002 & <0.002 & <0.002 & 0.28 & 0.0001\end{array}$ $\begin{array}{lllllllllllll}<0.001 & <0.01 & 0.004 & --- & 0.058 & <0.002 & <0.0002 & <0.002 & <0.002 & <0.002 & <0.002 & 0.03 & <0.0001\end{array}$ $\begin{array}{lllllllllllllll}<0.001 & <0.01<0.001 & <0.002 & 0.028 & <0.002 & <0.0002 & <0.002 & <0.002 & 0.047 & <0.002 & <0.01 & <0.0001\end{array}$ $<0.001<0.01<0.001<0.002 \quad 0.008<0.002<0.0002<0.002 \quad 0.004<0.002<0.002<0.01<0.0001$

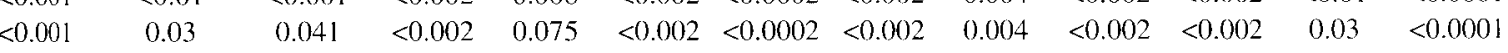
$\begin{array}{lllllllllllll}<0.001 & 0.03 & 0.004 & <0.002 & 0.012 & <0.002 & <0.0002 & <0.002 & <0.002 & 0.12 & <0.002 & <0.01 & <0.0001\end{array}$ $<0.001<0.02<0.001<0.002 \quad 0.019<0.002<0.0002<0.002<0.002<0.002<0.002<0.09<0.0001$

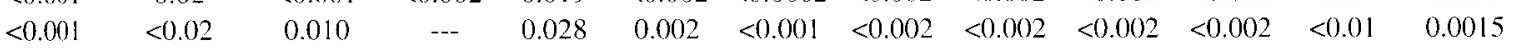

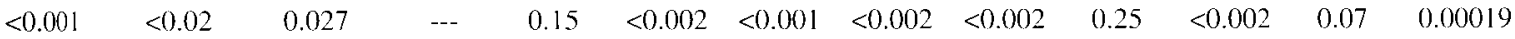
$\begin{array}{lllllllllllll}<0.001 & <0.02 & 0.0072 & - & 0.12 & <0.002<0.001<0.002 & <0.002 & 0.039 & 0.003<0.01 & 0.00007\end{array}$ $\begin{array}{lllllllllllll}<0.001 & <0.02 & 0.011 & -- & 0.062 & <0.002 & <0.001 & <0.002 & <0.002 & 0.009 & 0.003 & <0.01 & 0.00030\end{array}$ $\begin{array}{lllllllllllll}<0.001 & <0.02 & 0.0090 & --- & 0.060 & <0.002 & <0.001 & <0.002 & <0.002 & 0.11 & 0.002 & 0.21 & 0.00031\end{array}$ $<0.001<\begin{array}{llllllllllll}0.02 & 0.032 & -. & 0.088 & <0.002 & <0.001 & <0.002 & 0.004 & <0.002 & 0.003 & <0.01 & 0.00015\end{array}$ $\begin{array}{lllllllllllll}<0.001 & 0.13 & 0.0013 & -. & 0.011 & <0.002 & <0.001 & <0.002 & <0.002 & 0.10 & 0.003 & 0.09 & <0.00005\end{array}$ $\begin{array}{lllllllllllll}<0.001 & 0.04 & 0.011 & --- & 0.10 & <0.002 & <0.001 & <0.002 & <0.002 & 0.24 & <0.002 & 0.01 & 0.00016\end{array}$

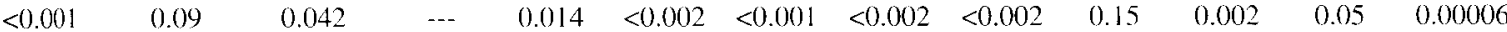
$\begin{array}{lllllllllllllllll}<0.001 & <0.02 & 0.013 & \ldots & 0.023 & <0.002 & <0.001 & <0.002 & <0.002 & <0.002 & <0.002 & <0.01 & 0.00015\end{array}$ $\begin{array}{llllllllllllll}<0.001 & <0.02 & 0.18 & \ldots & 0.021 & <0.002 & <0.001 & <0.002 & <0.002 & <0.002 & <0.002 & <0.01 & 0.00008\end{array}$

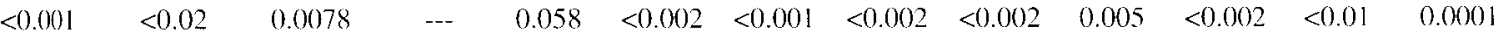
$<0.001<0.02 \quad 0.0079 \quad-0.030<0.002<0.001<0.002<0.002<0.002 \quad 0.002<0.01 \quad 0.0001$ $\begin{array}{lllllllllllll}<0.001 & <0.02 & 0.0034 & --- & 0.005 & <0.002 & <0.001 & <0.002 & <0.002 & 0.003 & <0.002 & 0.02 & 0.0001\end{array}$

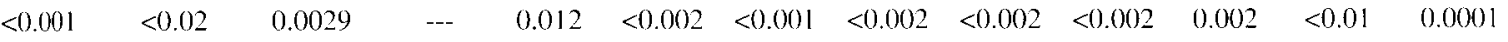
$<0.001<<<0.02 \quad 0.0012 \quad---0.015<0.002<0.001<0.002<0.002<0.002<0.002<0.01 \quad 0.0002$ $<0.001 \quad<0.02 \quad<0.0002 \quad---\quad 00.045 \quad<0.002 \quad<0.001 \quad<0.002 \quad<0.002 \quad<0.002 \quad<0.002 \quad<0.01 \quad 0.0001$

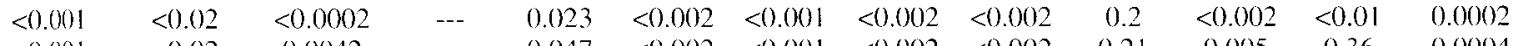

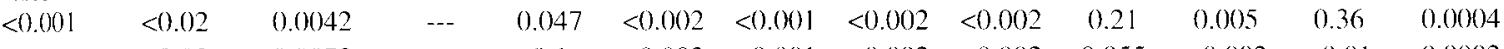

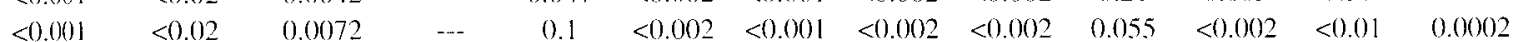


Table 5: Continued

$\begin{array}{ll}\text { Sample } & \text { Name or Description } \\ \text { Background Wells } & \\ \text { DV97-49 } & \text { Hole in the Wall \#2 Well } \\ \text { DV97-57 } & \text { Bolivia Artesian Well } \\ \text { DV97-70 } & \text { Flowing well @ AA Tank } \\ \text { DV97-7I } & \text { Shaw Well } \\ \text { DV98-115 } & \text { Irrigation Well } \\ \text { DV98-116 } & \text { Brinkerhoff Well } \\ \text { DV98-172 } & \text { Bernice Well } \\ & \\ \text { Background Streams/Rain } \\ \text { DV97-58 } & \text { Cottonwood Creek } \\ \text { DV98-107 } & \text { Unnamed Ck by Stu's Seep } \\ \text { DV98-110 } & \text { Cottonwood Creek } \\ \text { DV98-119 } & \text { Unnamed Stream } \\ \text { DV98-121 } & \text { White Rock Canyon } \\ \text { DV98-125 } & \text { Rain, Lizard Well Tank } \\ \text { DV98-126 } & \text { Home Station Wash } \\ \text { DV98-127 } & \text { Cedar Canyon Wash } \\ \text { DV98-130 } & \text { Bucher Creek } \\ \text { DV98-171 } & \text { Not-So-OK Creek } \\ \text { DV98-173 } & \text { Bernice Creek } \\ \text { DV98-174 } & \text { Hoyt Creek } \\ \text { DV98-180 } & \text { Mt. Augusta Creek } \\ \text { DV99-213 } & \text { Dixie Salt Lake } \\ & \\ \text { Fumarole Condensates } \\ \text { DV97-43 } & \text { Crack 4 Fumarole } \\ \text { DV97-44 } & \text { Senator Fumarole } \\ \text { DV98-108 } & \text { Senator Fumarole } \\ \text { DV98-164 } & \text { Calcite Fumarole } \\ \text { DV98-109 } & \text { Senator Fumarole } \\ \text { DV98-165 } & \text { Calcite Fumarole } \\ \text { DV98-166 } & \text { South Bench Fumarole } \\ & \end{array}$

\begin{tabular}{|c|c|c|c|c|c|c|c|c|c|c|c|c|c|}
\hline Date & Ag & Al (Total) & As & Au & $\mathrm{Ba}$ & $\mathrm{Be}$ & Cd & Co & $\mathrm{Cr}$ & Cs & $\mathrm{Cu}$ & $\mathrm{Fe}$ & $\mathrm{Hg}$ \\
\hline $11 / 04 / 97$ & $<0.001$ & 0.01 & $<0.001$ & --- & 0.022 & $<0.002$ & 0.0003 & $<0.002$ & $<0.002$ & $<0.002$ & 0.01 & $<0.01$ & 0.0002 \\
\hline $11 / 05 / 97$ & $<0.001$ & $<0.01$ & 0.027 & $<0.002$ & 0.036 & $<0.002$ & $<0.0002$ & $<0.002$ & 0.002 & 0.021 & 0.003 & 0.43 & 0.0003 \\
\hline $11 / 07 / 97$ & $<0.001$ & $<0.01$ & 0.022 & $<0.002$ & 0.012 & $<0.002$ & $<0.0002$ & $<0.002$ & 0.003 & $<0.002$ & $<0.002$ & $<0.01$ & $<0.0001$ \\
\hline $11 / 07 / 97$ & $<0.001$ & 0.04 & 0.029 & $<0.002$ & 0.014 & $<0.002$ & $<0.0002$ & $<0.002$ & 0.004 & $<0.002$ & $<0.002$ & $<0.01$ & $<0.0001$ \\
\hline $05 / 04 / 98$ & $<0.001$ & $<0.02$ & 0.0067 & --- & 0.054 & $<0.002$ & $<0.001$ & $<0.002$ & 0.002 & 0.025 & 0.004 & $<0.01$ & 0.00008 \\
\hline $05 / 04 / 98$ & $<0.001$ & $<0.02$ & 0.0052 & --- & 0.080 & $<0.002$ & $<0.001$ & $<0.002$ & 0.003 & $<0.002$ & 0.004 & 0.01 & 0.00010 \\
\hline $10 / 27 / 98$ & $<0.001$ & $<0.02$ & 0.0008 & --- & 0.002 & $<0.002$ & $<0.001$ & $<0.002$ & $<0.002$ & $<0.002$ & $<0.002$ & 0.43 & 0.0002 \\
\hline $11 / 05 / 97$ & $<0.001$ & $<0.01$ & 0.004 & -- & 0.036 & $<0.002$ & $<0.0002$ & $<0.002$ & $<0.002$ & $<0.002$ & $<0.002$ & $<0.01$ & $<0.0001$ \\
\hline $04 / 30 / 98$ & $<0.001$ & $<0.02$ & 0.010 & --- & 0.11 & $<0.002$ & $<0.001$ & $<0.002$ & $<0.002$ & $<0.002$ & 0.003 & 0.01 & 0.00035 \\
\hline $05 / 01 / 98$ & $<0.001$ & 0.19 & 0.023 & --- & 0.044 & --- & $<0.001$ & $<0.002$ & 0.003 & $<0.002$ & $<0.002$ & 0.18 & 0.00024 \\
\hline $05 / 05 / 98$ & $<0.001$ & 0.31 & 0.0048 & --- & 0.038 & $<0.002$ & $<0.001$ & $<0.002$ & $<0.002$ & $<0.002$ & 0.005 & 0.35 & 0.000007 \\
\hline $05 / 05 / 98$ & $<0.001$ & $<0.02$ & 0.0050 & --- & 0.038 & $<0.002$ & $<0.001$ & $<0.002$ & $<0.002$ & $<0.002$ & 0.003 & 0.02 & $<0.00005$ \\
\hline $05 / 06 / 98$ & $<0.001$ & $<0.02$ & 0.0043 & $-\cdots$ & 0.004 & $<0.002$ & $<0.001$ & $<0.002$ & 0.005 & 0.003 & 0.018 & 0.75 & $<0.00005$ \\
\hline $05 / 06 / 98$ & $<0.001$ & 0.28 & 0.0044 & --- & 0.021 & $<0.002$ & $<0.001$ & $<0.002$ & $<0.002$ & $<0.002$ & 0.004 & 0.25 & 0.00006 \\
\hline $05 / 06 / 98$ & $<0.001$ & 0.52 & 0.0010 & --- & 0.023 & $<0.002$ & $<0.001$ & $<0.002$ & $<0.002$ & $<0.002$ & 0.003 & 0.38 & 0.00005 \\
\hline $05 / 06 / 98$ & $<0.001$ & 0.02 & 0.0020 & --- & 0.044 & $<0.002$ & $<0.001$ & $<0.002$ & $<0.002$ & $<0.002$ & $<0.002$ & 0.03 & $<0.00005$ \\
\hline $10 / 27 / 98$ & $<0.001$ & $<0.02$ & 0.0026 & --- & 0.029 & $<0.002$ & $<0.001$ & $<0.002$ & $<0.002$ & $<0.002$ & $<0.002$ & $<0.01$ & 0.0001 \\
\hline $10 / 27 / 98$ & $<0.001$ & $<0.02$ & 0.0065 & --- & 0.029 & $<0.002$ & $<0.001$ & $<0.002$ & $<0.002$ & $<0.002$ & $<0.002$ & $<0.01$ & 0.0003 \\
\hline $10 / 27 / 98$ & $<0.001$ & $<0.02$ & 0.025 & $-\cdots$ & 0.030 & $<0.002$ & $<0.001$ & $<0.002$ & $<0.002$ & $<0.002$ & $<0.002$ & $<0.01$ & 0.0001 \\
\hline $10 / 28 / 98$ & $<0.001$ & $<0.02$ & 0.0030 & --- & 0.029 & $<0.002$ & $<0.001$ & $<0.002$ & $<0.002$ & $<0.002$ & $<0.002$ & $<0.01$ & 0.0001 \\
\hline $05 / 10 / 99$ & $<0.001$ & $<0.02$ & 0.18 & -- & 0.03 & $<0.002$ & $<0.001$ & $<0.002$ & $<0.002$ & $<0.01$ & $<0.002$ & 0.05 & 0.0004 \\
\hline $11 / 03 / 97$ & --- & 0.08 & $<0.001$ & --- & --- & $<0.002$ & --- & --- & --- & --- &..- & 0.02 & 0.009 \\
\hline $11 / 03 / 97$ & $<0.001$ & 3.15 & 0.003 & --- & $\ldots$ & $<0.002$ & $<0.0002$ & $<0.002$ & 0.006 & $<0.002$ & $<0.002$ & 1.85 & 0.056 \\
\hline $05 / 01 / 98$ & --- & --- & $<0.0002$ & --- & -- & $<0.002$ & --- & --- & --- & --- & --- & --- & 0.0026 \\
\hline $05 / 01 / 98$ & --- & --- & 0.014 & $\cdots$ & $\cdots$ & $<0.002$ & --- & --- & $\ldots$ & --- & --- & --- & 0.0100 \\
\hline $10 / 24 / 98$ & --- & 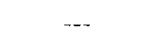 & 0.0023 & -- & --- & --- & --- & --- & --- & --- & --- & --- & 0.0040 \\
\hline $10 / 25 / 98$ & -- & --- & 0.0066 & $\ldots$ &.-- & --- & -... & -- & --- & --- & --- & --- & 0.0104 \\
\hline $10 / 26 / 98$ & $\ldots$ & --- & 0.0093 & --- & ... & -.- & -.- & -.- & --- & ... & $\ldots$ & ... & 0.0008 \\
\hline
\end{tabular}


Table 5: Continued

\begin{tabular}{|c|c|c|c|c|c|c|c|c|c|c|c|c|c|c|c|c|c|c|c|}
\hline $\begin{array}{l}\text { Sample } \\
\text { Brines }\end{array}$ & I & Mn & Mo & $\mathbf{N H}_{4}$ & $\mathbf{N i}$ & $\mathrm{NO}_{2}$ & $\mathrm{NO}_{3}$ & $\mathbf{P b}$ & $\mathbf{P O}_{4}$ & $\mathbf{R b}$ & $\mathbf{S}$ & Sb & Se & $\mathbf{S}_{2} \mathbf{O}_{3}$ & $\mathrm{SO}_{3}$ & $\mathbf{T i}$ & $\mathrm{Tl}$ & V & $\mathrm{Zn}$ \\
\hline DIXEI02-W & --- & $<0.01$ & --- & 1.46 & --- & $<0.05$ & $<0.05$ & $\ldots$ & $<0.1$ & 0.71 & $\ldots$ & --- & -.- & --- & 0.97 & -.. & --- & --- & $\ldots$ \\
\hline DV96-8 & 0.043 & $<0.002$ & 0.04 & 2.13 & $<0.002$ & $<0.04$ & 0.19 & $<0.002$ & $<0.1$ & 0.53 & 0.6 & 0.088 & $<0.0001$ & 1.38 & 0.11 & $<0.002$ & --- & 0.013 & $<0.01$ \\
\hline DV96-9 & 0.03 & $<0.002$ & 0.06 & 1.84 & $<0.002$ & $<0.04$ & $<0.04$ & $<0.002$ & $<0.1$ & 0.55 & 1.84 & 0.069 & $<0.0001$ & 2.3 & 0.43 & $<0.002$ & --- & 0.009 & $<0.01$ \\
\hline DV97-11 & 0.058 & $<0.002$ & 0.1 & 1.88 & $<0.002$ & $<0.05$ & $<0.05$ & 0.002 & $<0.1$ & 0.61 & 11.3 & 0.0082 & $<0.001$ & 2.3 & 0.33 & $<0.002$ & $<0.005$ & 0.011 & $<0.01$ \\
\hline DV97-13 & 0.051 & $<0.002$ & 0.08 & 1.82 & $<0.002$ & $<0.05$ & $<0.05$ & 0.002 & $<0.1$ & 0.69 & 14.3 & 0.0013 & $<0.00 \mathrm{~J}$ & 2.87 & 0.43 & $<0.002$ & $<0.005$ & 0.012 & 0.01 \\
\hline DV97-14 & 0.057 & $<0.002$ & 0.11 & 1.86 & $<0.002$ & $<0.05$ & $<0.05$ & 0.002 & $<0.1$ & 0.64 & 12.2 & 0.0069 & $<0.001$ & 2.51 & 0.65 & $<0.002$ & $<0.005$ & 0.009 & $<0.01$ \\
\hline DV97-16 & 0.055 & $<0.002$ & 0.12 & 1.81 & 0.033 & $<0.05$ & $<0.05$ & 0.002 & $<0.1$ & $0.6 !$ & 10.8 & 0.0046 & $<0.001$ & 2.53 & 0.22 & $<0.002$ & $<0.005$ & 0.011 & $<0.01$ \\
\hline DV97-18 & 0.049 & $<0.002$ & 0.09 & 1.67 & $<0.002$ & $<0.05$ & $<0.05$ & $<0.002$ & $<0.1$ & 0.61 & 13.2 & 0.0005 & $<0.001$ & 2.69 & 0.36 & $<0.002$ & $<0.005$ & 0.011 & $<0.01$ \\
\hline DV97-20 & 0.054 & $<0.002$ & 0.07 & 1.62 & $<0.002$ & $<0.05$ & $<0.05$ & 0.002 & $<0.1$ & 0.64 & 12.5 & 0.0092 & $<0.001$ & 2.75 & 0.24 & $<0.002$ & $<0.005$ & 0.011 & $<0.01$ \\
\hline DV97-23 & 0.055 & $<0.002$ & 0.07 & 1.76 & $<0.002$ & $<0.05$ & $<0.05$ & 0.002 & $<0.1$ & 0.62 & 13.9 & 0.0036 & $<0.001$ & 2.79 & 0.23 & $<0.002$ & $<0.005$ & 0.011 & 0.02 \\
\hline DV97-25 & 0.033 & $<0.002$ & 0.023 & 1.79 & $<0.002$ & $<0.05$ & $<0.05$ & 0.002 & $<0.1$ & 0.57 & 25.7 & 0.011 & $<0.001$ & 4.07 & 0.93 & $<0.002$ & $<0.005$ & 0.009 & $<0.01$ \\
\hline DV97-26 & 0.032 & $<0.002$ & 0.015 & 1.67 & $<0.002$ & $<0.05$ & $<0.05$ & 0.002 & $<0.1$ & 0.61 & 23.2 & 0.02 & $<0.001$ & 3.68 & 0.71 & $<0.002$ & $<0.005$ & 0.011 & $<0.01$ \\
\hline DV97-29 & 0.04 & $<0.002$ & 0.023 & 1.64 & $<0.002$ & $<0.05$ & $<0.05$ & $<0.002$ & $<0.1$ & 0.58 & 18.9 & 0.005 & $<0.001$ & 3.61 & 0.63 & $<0.002$ & $<0.005$ & 0.011 & $<0.01$ \\
\hline DV97-30 & 0.036 & $<0.002$ & 0.026 & 1.63 & $<0.002$ & $<0.05$ & $<0.05$ & 0.002 & $<0.1$ & 0.61 & 17.5 & 0.0086 & $<0.001$ & 3.74 & 0.94 & $<0.002$ & $<0.005$ & 0.01 & $<0.01$ \\
\hline DV98-73 & 0.02 & $<0.002$ & 0.06 & 1.51 & $<0.002$ & $<0.05$ & $<0.05$ & $<0.002$ & $<0.1$ & 0.62 & 0.33 & --- & --- & 3.79 & 0.60 & $<0.002$ & -- & 0.009 & $<0.01$ \\
\hline DV98-75 & 0.02 & $<0.002$ & 0.03 & 1.54 & $<0.002$ & $<0.05$ & $<0.05$ & $<0.002$ & $<0.1$ & 0.55 & 1.17 & --- & --- & 4.73 & 0.42 & $<0.002$ & $-\cdots$ & 0.007 & $<0.01$ \\
\hline DV98-77 & 0.02 & $<0.002$ & 0.05 & 1.51 & $<0.002$ & $<0.05$ & $<0.05$ & $<0.002$ & $<0.1$ & 0.57 & 1.29 & --- & --- & 3.52 & 0.25 & $<0.002$ & $-\cdots$ & 0.009 & $<0.01$ \\
\hline DV98-79 & 0.03 & $<0.002$ & 0.05 & 1.49 & $<0.002$ & $<0.05$ & $<0.05$ & $<0.002$ & $<0.1$ & 0.62 & 1.49 & --- & --- & 7.14 & 0.26 & $<0.002$ & $\ldots$ & 0.008 & $<0.01$ \\
\hline DV98-80 & 0.03 & $<0.002$ & 0.05 & 1.56 & $<0.002$ & $<0.05$ & $<0.05$ & $<0.002$ & $<0.1$ & 0.62 & 0.25 & --- & --- & 2.31 & $<0.05$ & $<0.002$ & -- & 0.011 & $<0.01$ \\
\hline DV98-82 & 0.03 & $<0.002$ & 0.05 & 1.68 & $<0.002$ & $<0.05$ & $<0.05$ & $<0.002$ & $<0.1$ & 0.58 & 0.33 & --- & --- & 2.27 & $<0.05$ & $<0.002$ & -- & 0.009 & $<0.01$ \\
\hline DV98-84 & 0.03 & $<0.002$ & 0.06 & 1.97 & $<0.002$ & $<0.05$ & $<0.05$ & $<0.002$ & $<0.1$ & 0.60 & 0.40 & --- & $\ldots$ & 2.36 & $<0.05$ & $<0.002$ & -- & 0.008 & $<0.01$ \\
\hline DV98-86 & 0.03 & $<0.002$ & 0.04 & 1.98 & $<0.002$ & $<0.05$ & $<0.05$ & $<0.002$ & $<0.1$ & 0.62 & 0.20 & --- & --- & 2.35 & $<0.05$ & $<0.002$ &.-- & 0.010 & $<0.01$ \\
\hline DV98-88 & 0.03 & $<0.002$ & 0.08 & 2.15 & $<0.002$ & $<0.05$ & $<0.05$ & $<0.002$ & $<0.1$ & 0.62 & 0.32 & --- & --- & 3.71 & $<0.05$ & $<0.002$ & -- & 0.008 & $<0.01$ \\
\hline DV98-90 & 0.03 & $<0.002$ & 0.06 & 1.69 & $<0.002$ & $<0.05$ & $<0.05$ & $<0.002$ & $<0.1$ & 0.62 & 0.61 & --- & --- & 3.68 & $<0.05$ & $<0.002$ & -.. & 0.009 & $<0.01$ \\
\hline DV98-92 & 0.03 & $<0.002$ & 0.07 & 1.83 & $<0.002$ & $<0.05$ & $<0.05$ & $<0.002$ & $<0.1$ & 0.60 & 0.46 & --- & --- & 2.57 & $<0.05$ & $<0.002$ & -- & 0.009 & $<0.01$ \\
\hline DV98-95 & 0.03 & $<0.002$ & 0.03 & 1.63 & $<0.002$ & $<0.05$ & $<0.05$ & $<0.002$ & $<0.1$ & 0.55 & 0.25 & --- & --- & 2.88 & $<0.05$ & $<0.002$ & -- & 0.008 & $<0.01$ \\
\hline DV98-133 & 0.03 & $<0.002$ & 0.039 & 1.94 & $<0.002$ & $<0.05$ & 0.17 & $<0.002$ & $<0.1$ & 0.56 & 2.51 & --- & --- & 4.02 & $<0.1$ & $<0.002$ & $-\cdots$ & 0.011 & $<0.01$ \\
\hline DV98-135 & 0.04 & $<0.002$ & 0.068 & 1.20 & $<0.002$ & $<0.05$ & 0.45 & 0.002 & $<0.1$ & 0.47 & 1.44 & --- & --- & 2.60 & $<0.1$ & $<0.002$ & $-\cdots$ & 0.011 & $<0.01$ \\
\hline DV98-138 & 0.03 & $<0.002$ & 0.056 & 1.99 & $<0.002$ & $<0.05$ & $<0.05$ & $<0.002$ & $<0.1$ & 0.53 & 1.83 & --- & --- & 3.50 & $<0.1$ & $<0.002$ & -- & 0.008 & $<0.01$ \\
\hline DV98-140 & 0.03 & $<0.002$ & 0.049 & 1.60 & $<0.002$ & $<0.05$ & $<0.05$ & $<0.002$ & $<0.1$ & 0.63 & 2.47 & ... & --- & 3.7 & $<0.1$ & $<0.002$ & -.. & 0.008 & $<0.01$ \\
\hline DV98-141 & 0.03 & $<0.002$ & 0.032 & 1.58 & $<0.002$ & $<0.05$ & $<0.05$ & 0.002 & $<0.1$ & 0.57 & 1.99 & --- & --- & 3.85 & $<0.1$ & $<0.002$ & $-\cdots$ & 0.008 & $<0.01$ \\
\hline DV98-145 & 0.04 & 0.003 & 0.048 & 1.81 & $<0.002$ & $<0.05$ & 0.12 & $<0.002$ & $<0.1$ & 0.56 & 0.63 & --- & --- & 2.38 & $<0.1$ & $<0.002$ & -- & 0.010 & $<0.01$ \\
\hline DV98-147 & 0.04 & $<0.002$ & 0.052 & 1.88 & $<0.002$ & $<0.05$ & $<0.05$ & $<0.002$ & $<0.1$ & 0.57 & 0.66 & --- & --- & 2.22 & $<0.1$ & $<0.002$ & -- & 0.010 & $<0.01$ \\
\hline DV98-148 & 0.05 & $<0.002$ & 0.086 & 1.89 & $<0.002$ & $<0.05$ & $<0.05$ & $<0.002$ & $<0.1$ & 0.57 & 0.42 & --- & --- & 2.50 & $<0.1$ & $<0.002$ & --- & 0.010 & $<0.01$ \\
\hline DV98-150 & 0.04 & $<0.002$ & 0.079 & 1.89 & $<0.002$ & $<0.05$ & $<0.05$ & $<0.002$ & $<0.1$ & 0.58 & 1.08 & & --- & 2.41 & $<0.1$ & $<0.002$ & --- & 0.010 & $<0.01$ \\
\hline DV98-152 & 0.05 & $<0.002$ & 0.095 & 2.18 & $<0.002$ & $<0.05$ & $<0.05$ & $<0.002$ & $<0.1$ & 0.57 & 0.75 & --- & --- & 2.31 & $<0.1$ & $<0.002$ & -- & 0.011 & $<0.01$ \\
\hline DV98-154 & 0.04 & $<0.002$ & 0.072 & 2.37 & $<0.002$ & $<0.05$ & $<0.05$ & $<0.002$ & $<0.1$ & 0.58 & 0.73 & --- & -.. & 2.08 & $<0.1$ & $<0.002$ & ... & 0.009 & 0.03 \\
\hline DV98-156 & 0.04 & $<0.002$ & 0.091 & 1.70 & $<0.002$ & $<0.05$ & $<0.05$ & $<0.002$ & $<0.1$ & 0.57 & 1.1 .3 & --- & --- & 2.98 & $<0.1$ & $<0.001$ & $\ldots$ & 0.009 & $<0.01$ \\
\hline DV98-159 & 0.04 & $<0.002$ & 0.094 & 1.88 & $<0.002$ & $<0.05$ & $<0.05$ & $<0.002$ & $<0.1$ & 0.56 & 0.89 & --- & --- & 2.87 & $<0.1$ & $<0.002$ & $\ldots$ & 0.012 & $<0.01$ \\
\hline DV99-182 & 0.04 & $<0.002$ & 0.05 & 1.78 & $<0.002$ & $<0.05$ & $<0.05$ & $<0.002$ & $<0.1$ & 0.59 & 0.37 & $\ldots$ & --- & 1.74 & $<0.1$ & $<0.002$ & --- & 0.013 & $<0.01$ \\
\hline
\end{tabular}


Table 5: Continued

\begin{tabular}{|c|c|c|c|c|c|c|c|c|c|c|c|c|c|c|c|c|c|c|c|}
\hline Sample & I & Mn & Mo & $\mathbf{N H}_{4}$ & $\mathbf{N i}$ & $\mathrm{NO}_{2}$ & $\mathrm{NO}_{3}$ & $\mathbf{P b}$ & $\mathbf{P O}_{4}$ & $\mathbf{R b}$ & $\mathbf{S}$ & Sb & $\mathrm{Se}$ & $\mathrm{S}_{2} \mathrm{O}_{3}$ & $\mathbf{S O}_{3}$ & $\mathrm{Ti}$ & $\mathrm{TI}$ & $\mathbf{V}$ & $\mathbf{Z n}$ \\
\hline DV99-184 & 0.04 & $<0.002$ & 0.06 & 1.84 & $<0.002$ & $<0.05$ & $<0.05$ & $<0.002$ & $<0.1$ & 0.63 & 0.37 & --- & $\ldots$ & 2.06 & $<0.1$ & $<0.002$ & --- & 0.011 & $<0.01$ \\
\hline DV99-186 & 0.04 & $<0.002$ & 0.06 & 1.7 & $<0.002$ & $<0.05$ & $<0.05$ & $<0.002$ & $<0.1$ & 0.57 & 0.37 & --- & --- & 1.98 & $<0.1$ & $<0.002$ & --- & 0.011 & $<0.01$ \\
\hline DV99-188 & 0.04 & $<0.002$ & 0.07 & 1.9 & $<0.002$ & $<0.05$ & $<0.05$ & $<0.002$ & $<0.1$ & 0.65 & 0.37 & --- & --- & 1.41 & $<0.1$ & $<0.002$ & --- & 0.01 & $<0.01$ \\
\hline DV99-190 & 0.04 & $<0.002$ & 0.09 & 1.93 & $<0.002$ & $<0.05$ & $<0.05$ & $<0.002$ & $<0.1$ & 0.62 & 0.37 & --- & --- & 2.11 & $<0.1$ & $<0.002$ & --- & 0.012 & $<0.01$ \\
\hline DV99-194 & 0.04 & $<0.002$ & 0.05 & 2.05 & $<0.002$ & $<0.05$ & $<0.05$ & $<0.002$ & $<0.1$ & 0.63 & 0.37 & --- & --- & 2.3 & $<0.1$ & $<0.002$ & --- & 0.013 & $<0.01$ \\
\hline DV99-196 & 0.04 & $<0.002$ & 0.08 & 1.74 & $<0.002$ & $<0.05$ & $<0.05$ & $<0.002$ & $<0.1$ & 0.62 & 0.37 & --- & -- & 2.66 & $<0.1$ & $<0.002$ & --- & 0.011 & $<0.01$ \\
\hline DV99-197 & 0.04 & $<0.002$ & 0.06 & 1.92 & $<0.002$ & $<0.05$ & $<0.05$ & $<0.002$ & $<0.1$ & 0.63 & 0.37 & --- & --- & 2.63 & $<0.1$ & $<0.002$ & --- & 0.012 & $<0.01$ \\
\hline DV99-199 & 0.02 & $<0.002$ & 0.06 & 1.66 & $<0.002$ & $<0.05$ & $<0.05$ & $<0.002$ & $<0.1$ & 0.61 & 0.37 & -- & - & 3.91 & $<0.1$ & $<0.002$ & --- & 0.009 & $<0.01$ \\
\hline DV99-200 & 0.02 & $<0.002$ & 0.06 & 1.66 & $<0.002$ & $<0.05$ & $<0.05$ & $<0.002$ & $<0.1$ & 0.62 & 0.37 & --- & --- & 3.74 & $<0.1$ & $<0.002$ & --- & 0.01 & $<0.01$ \\
\hline DV99-204 & 0.02 & 0.002 & 0.06 & 1.75 & $<0.002$ & $<0.05$ & $<0.05$ & $<0.002$ & $<0.1$ & 0.65 & 0.37 & --- & --- & 3.74 & $<0.1$ & $<0.002$ & --- & 0.01 & $<0.01$ \\
\hline DV74782786-brine 2 & 0.03 & $<0.002$ & 0.04 & 1.28 & $<0.002$ & $<0.05$ & $<0.05$ & $<0.002$ & $<0.1$ & 0.6 & -.. & --- & --- & $<0.01$ & $<0.1$ & $<0.002$ & --- & 0.008 & $<0.01$ \\
\hline DV76781986-brine 4 & 0.03 & $<0.002$ & 0.03 & 1.42 & $<0.002$ & $<0.05$ & $<0.05$ & $<0.002$ & $<0.1$ & 0.52 & -- & --- & --- & 0.18 & $<0.1$ & $<0.002$ &.-- & 0.007 & $<0.01$ \\
\hline DV453382186-brine 6 & 0.03 & $<0.002$ & 0.04 & 1.39 & $<0.002$ & $<0.05$ & $<0.05$ & $<0.002$ & $<0.1$ & 0.59 & --- & --- & --- & 0.96 & $<0.1$ & $<0.002$ & --- & 0.007 & $<0.01$ \\
\hline DV73782886-brine 8 & 0.03 & 0.002 & 0.04 & 1.6 & $<0.002$ & $<0.05$ & $<0.05$ & $<0.002$ & $<0.1$ & 0.56 & --- & --- & --- & 0.13 & $<0.1$ & $<0.002$ & --- & 0.008 & $<0.01$ \\
\hline DV321882686-brine 10 & 0.05 & 0.004 & 0.05 & 1.92 & $<0.002$ & $<0.05$ & $<0.05$ & $<0.002$ & $<0.1$ & 0.38 & --- & -- & -- & 0.05 & $<0.1$ & $<0.002$ & --- & 0.003 & $<0.01$ \\
\hline DV651882686-brine 12 & 0.04 & 0.003 & 0.04 & 1.63 & $<0.002$ & $<0.05$ & $<0.05$ & $<0.002$ & $<0.1$ & 0.31 & --- & --- & --- & 0.08 & $<0.1$ & $<0.002$ & --- & 0.008 & $<0.01$ \\
\hline No number & 0.15 & 0.047 & 0.02 & 0.1 & $<0.002$ & $<0.05$ & $<0.05$ & $<0.002$ & $<0.1$ & 0.035 & $\ldots$ & --- & --- & $<0.01$ & $<0.1$ & 0.019 & --- & 0.003 & $<0.0$ \\
\hline
\end{tabular}

\section{$\$ \quad$ Condensates}

DIXE102-

DV96-10

DV97-12

DV97-15

DV97-17

DV97-19

DV97-21

DV97-22

DV97-24

DV97-27

DV97-28

DV97-31

DV98-76

DV98-78

DV98-8

DV98-83

DV98-85

DV98-87

DV98-89

DV98-91 $\begin{array}{ccccccccc}--- & -- & -- & 11.4 & -- & <0.02 & <0.02 & --- & <0.05\end{array}$

$\begin{array}{lllllllll}<0.005 & 0.016 & <0.01 & 14.6 & 3.75 & <0.02 & <0.02 & 0.002 & <0.05\end{array}$

$<0.005 \quad 0.003 \quad<0.01 \quad 12.7 \quad<0.002<0.02 \quad<0$.

$\begin{array}{llllll}--- & -- & - & 11.6 & -- & <0.02<0.02\end{array}$

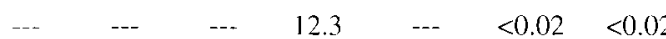

$\begin{array}{lllllll}-- & -- & -- & 13.3 & -. & <0.02 & <0.02\end{array}$

$\begin{array}{lllllll}-- & -- & - & 13.5 & -.- & <0.02 & <0.02\end{array}$

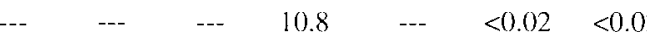

$\begin{array}{lllllll}-- & -- & -- & 9.51 \quad-- & <0.02 \quad<0.02\end{array}$

$\begin{array}{lllllll}-- & -- & -- & 12.8 & --- & <0.02 & <0.02\end{array}$

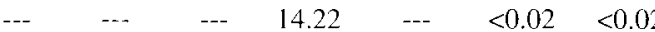

$\begin{array}{llllll}-- & -- & 13.9 & -- & <0.05 \quad<0.05\end{array}$

$\begin{array}{lllllll}-- & -- & -- & 13.1 & -- & <0.02 & <0.02\end{array}$

$\begin{array}{lllllll}-- & -- & -- & 12.3 & -- & <0.02 & <0.02\end{array}$

$\begin{array}{lllllll}-- & -- & -- & 13.4 & -- & <0.02 & <0.02\end{array}$

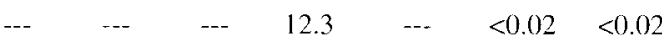

$\begin{array}{lllllll}--- & -- & -- & 13.8 & -- & <0.02 & <0.02\end{array}$

$\begin{array}{llllll}- & -- & 13.6 & - & - & <0.02<0.02\end{array}$

$\begin{array}{lllllll}-- & -- & -- & 15.7 & -- & <0.02 & <0.02\end{array}$

$\begin{array}{llllll}- & -- & 7.21 & -- & <0.02<0.02\end{array}$
$0.11<0.05$

$---<0.05$

$---<0.05$

$--\quad<0.05$

$---<0.05$

$--<<0.05$

--- 0.63

$---\quad<0.05$

$--\quad<0.1$

$\cdots<0.05$

$-<0.05$

$--<<0.05$

$--\quad<0.05$

$--\quad<0.05$

$--\quad<0.05$

$---<0.05$

$--\quad<0.05$

$--\quad<0.05$
-.. - 0.026

-.-

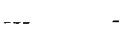

$---$

$--$

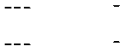

$---\quad$

$---$

$--$

$--$

$--$

$---$

\begin{tabular}{|c|c|c|c|c|c|c|c|}
\hline --- & --- & --- & 0.37 & --- & --- & --- & --- \\
\hline 0.0011 & $<0.0001$ & 3.67 & $<0.05$ & $<0.002$ & --- & $<0.002$ & 0.07 \\
\hline 0.0012 & $<0.0001$ & 3.53 & $<0.05$ & $<0.002$ & --- & $<0.002$ & 0.48 \\
\hline --- & --- & --- & 0.17 & -.. & --- & --- & $\cdots$ \\
\hline --- & --- & --- & 0.2 & $\ldots$ & --- & --- & --- \\
\hline --- & --- & --- & $<0.05$ & --- & --- & --- & -- \\
\hline --- & --- & --- & $<0.05$ & --- & --- & --- & $\cdots$ \\
\hline --- & --- & --- & 0.11 & --- & --- & --- & --- \\
\hline 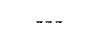 & --- & --- & $<0.05$ & $\ldots$ & --- & --- & --- \\
\hline-- & --- & --- & 0.37 & $\ldots$ & --- & --- & --- \\
\hline$\cdots$ & --- & --- & 0.35 & -.- & --- & --- & --- \\
\hline --- & $\ldots$ & --- & $<0.05$ & --- & -.. & --- & --- \\
\hline --- & --. & --. & $<0.05$ & -.- & --- & -.- & -- \\
\hline --- & --- & --- & 0.09 & $\ldots$ & --- & --- & --- \\
\hline --- & --- & --- & 0.81 & $\ldots$ & --- & --- & -- \\
\hline$\ldots$ & --- & --- & $<0.05$ & $-\cdots$ & --- & --- & $\cdots$ \\
\hline$\cdots$ & --- & --- & $<0.05$ & -.- & --- & --- & -- \\
\hline$\ldots$ & --- & --- & $<0.05$ & $\ldots$ & --- & --- & -- \\
\hline --- & --- & --- & $<0.05$ & $-\cdots$ & --- & --- & --- \\
\hline--- & --- & --- & 0.70 & $\cdots$ & $\cdots$ & --- & -- \\
\hline--- & --- & --- & $<0.05$ & $\ldots$ & --- & --- & --- \\
\hline --- & -.- & --- & 0.20 & $\ldots$ & --- & -.. & --- \\
\hline
\end{tabular}




\section{Table 5: Continued}

\begin{tabular}{|c|c|c|c|c|c|c|c|c|c|c|c|c|c|c|c|c|c|c|c|}
\hline Sample & $\mathbf{I}$ & Mn & Mo & $\mathbf{N H}_{4}$ & $\mathbf{N i}$ & $\mathrm{NO}_{2}$ & $\mathbf{N O}_{3}$ & $\mathrm{~Pb}$ & $\mathbf{P O}_{4}$ & $\mathbf{R b}$ & $\mathbf{S}$ & Sb & Se & $\mathbf{S}_{2} \mathbf{U}_{3}$ & $\mathrm{SO}_{3}$ & $\mathbf{T i}$ & TI & V & $\mathrm{Zn}$ \\
\hline DV98-93 & --- & --- & -- & 15.5 & --- & $<0.02$ & $<0.02$ & --- & $<0.05$ & --- & --- & $\ldots$ & --- & --- & $<0.05$ & --- & -- & --- & --- \\
\hline DV98-94 & --- & --- & --- & 14.2 & --- & $<0.02$ & $<0.02$ & --- & $<0.05$ & --- & --- & --- & $\cdots$ & --- & 0.78 & --- & --- & --- & --- \\
\hline DV98-101 & --- & --- & --- & 12.0 & --- & $<0.02$ & 0.03 & --- & $<0.05$ & --- & --- & --- & $\cdots$ & --- & 0.52 & --- & --- & --- & --- \\
\hline DV98-136 & --- & --- & --- & 13.0 & $\cdots$ & $<0.02$ & $<0.02$ & --- & $<0.05$ & --- & --- & --- & --- & $-\cdots$ & 0.19 & --- & --- & --- & --- \\
\hline DV98-137 & --- & --- & --- & 0.65 & $\ldots$ & $<0.02$ & $<0.02$ & --- & $<0.05$ & --- & --- & --- & --- & $\cdots-$ & 0.14 & --- & --- & --- & --- \\
\hline DV98-139 & --- & $\cdots$ & --- & 13.1 & --- & $<0.02$ & $<0.02$ & --- & $<0.05$ & --- & --- & -- & --- & --- & 0.06 & --- & --- & --- & --- \\
\hline DV98-142 & --- & $\cdots$ & --- & 12.8 & --- & $<0.02$ & $<0.02$ & --- & 0.07 & $\cdots$ & --- & --- & --- & --- & 0.08 & --- & --- & --- & $\cdots$ \\
\hline DV98-144 & --- & --- & --- & 13.5 & --- & $<0.02$ & 0.02 & $\cdots$ & $<0.05$ & --- & --- & --- & --- & --- & $<0.02$ & --- & --- & --- & --- \\
\hline DV98-146 & --- & --- & --- & 13.9 & --- & $<0.02$ & $<0.02$ & --- & $<0.05$ & --- & --- & --- & $\cdots$ & --- & $<0.02$ & --- & --- & --- & --- \\
\hline DV98-149 & --- & --- & $\cdots$ & 15.6 & --- & $<0.02$ & $<0.02$ & --- & $<0.05$ & --- & -- & --- & --- & --- & $<0.02$ & --- & --- & $\cdots$ & --- \\
\hline DV98-151 & --- & --- & --- & 14.9 & --- & $<0.02$ & 0.04 & --- & $<0.05$ & --- & --- & --- & --- & --- & 0.06 & --- & --- & -- & --- \\
\hline DV98-153 & --- & --- & $\cdots$ & 15.0 & --- & $<0.02$ & $<0.02$ & --- & $<0.05$ & --- & -.- & --- & --- & --- & $<0.02$ & --- & --- & -- & --- \\
\hline DV98-155 & --- & --- & --- & 10.3 & --- & $<0.02$ & $<0.02$ & --- & $<0.05$ & --- & $\cdots$ & --- & --- & --- & $<0.02$ & --- & --- & --- & --- \\
\hline DV98-157 & $\cdots$ & --- & --- & 11.3 & --- & $<0.02$ & $<0.02$ & --- & $<0.05$ & --- & --- & --- & --- & --- & 0.07 & --- & --- & --- & --- \\
\hline DV98-158 & $-\cdots$ & --- & --- & 13.9 & --- & $<0.02$ & $<0.02$ & --- & $<0.05$ & --- & --- & --- & --- & -- & 0.03 & --- & --- & --- & --- \\
\hline DV99-183 & --- & --- & --- & 14.9 & --- & $<0.01$ & $<0.01$ & --- & $<0.02$ & --- & --- & --- & --- & --- & $<0.02$ & --- & $\ldots$ & --- & --- \\
\hline DV99-185 & --- & --- & --- & 13.9 & --- & $<0.01$ & $<0.01$ & --- & $<0.02$ & --- & ..- & --- & --- & --- & $<0.02$ & --- & --- & --- & --- \\
\hline DV99- 187 & --- & $\ldots$ & --- & 13.9 & --- & $<0.01$ & $<0.01$ & --- & $<0.02$ & --- & --- & --- & --- & --- & $<0.02$ & --- & --- & --- & --- \\
\hline DV99-189 & --- & --- & --- & 16.2 & --- & $<0.01$ & $<0.01$ & --- & $<0.02$ & --- &.-- & --- & --- & --- & 0.18 & --- & --- & --- & --- \\
\hline DV99-191 & --- & --- & --- & 15 & --- & $<0.01$ & $<0.01$ & --- & $<0.02$ & --- & --- & --- & --- & --- & $<0.02$ & $\cdots$ & --- & $\cdots$ & --- \\
\hline DV99-192 & $\cdots$ & --- & --- & 14 & --- & $<0.01$ & $<0.01$ & --- & $<0.02$ & --- & --- & --- & --- & $\cdots$ & $<0.02$ & --- & --- & --- & --- \\
\hline DV99-193 & --- & --- & --- & 15.6 & -- & $<0.01$ & 0.02 & --- & $<0.02$ & --- & --- & $--\cdot$ & --- & --- & $<0.02$ & --- & -- & --- & --- \\
\hline DV99-195 & --- & --- & --- & 14.2 & --- & $<0.01$ & $<0.01$ & --- & $<0.02$ & --- & --- & $\cdots$ & --- & --- & $<0.02$ & --- & $\cdots$ & --- & $\cdots$ \\
\hline DV99-201 & --- & $-\cdots$ & --- & 11.5 & --- & $<0.01$ & $<0.01$ & --- & $<0.02$ & --- & --- & $-\cdots$ & --- & --- & 0.33 & --- & --- & --- & --- \\
\hline DV99-202 & --- & --- & --- & 12.8 & --- & $<0.01$ & $<0.01$ & --- & $<0.02$ & --- & --- & --- & --- & --- & $<0.02$ & --- & --- & --- & --- \\
\hline DV99-203 & --- & --- & --- & 13.4 & --- & $<0.01$ & $<0.01$ & --- & $<0.02$ & -- & --- & --- & --- & --- & $<0.02$ & --- & --- & --- & --- \\
\hline DV74782786-cond 1 & --- & --- & --- & 13 & --- & $<0.01$ & $<0.01$ & --- & $<0.02$ & --- & --- & --- & --- & --- & $<0.02$ & --- & --- & --- & --- \\
\hline DV76781986-cond 3 & --- & -.. & --- & 13.8 & $-\cdots$ & $<0.01$ & $<0.01$ & --- & $<0.02$ & $\cdots$ & --- & --- & --- & --- & $<0.02$ & --- & --- & --- & --- \\
\hline DV453382886-cond 5 & --- & $\cdots$ & --- & 11.9 & $\cdots$ & $<0.01$ & $<0.01$ & --- & $<0.02$ & -- & --- & --- & --- & --- & $<0.02$ & --- & --- & --- & --- \\
\hline DV73782886-cond 7 & --- & --- & --- & 12.8 & $\cdots$ & $<0.01$ & $<0.01$ & $-\cdots$ & $<0.02$ & --- & --- & --- & -- & --- & $<0.02$ & --- & --- & --- & --- \\
\hline DV321882686-cond 9 & --- & --- & -- & 17.2 & --- & $<0.01$ & $<0.01$ & --- & $<0.02$ & --- & $-\cdots$ & --- & $\cdots$ & --- & $<0.02$ & --- & --- & --- & --- \\
\hline DV651882686-cond 11 & -- & --- &.-- & 15.6 & -- & $<0.01$ & $<0.01$ & --- & $<0.02$ & --- & --- & --- & --- & --- & $<0.02$ & --- & --- & --- & --- \\
\hline \multicolumn{20}{|c|}{ Injection Well/Power Plant Fluids } \\
\hline DV96-2 & $<0.005$ & 0.012 & 0.02 & 26.3 & $<0.002$ & 0.03 & 43.5 & $<0.002$ & $<0.05$ & $<0.002$ & 0.04 & $<0.0001$ & 0.0003 & $<0.01$ & $<0.05$ & $<0.002$ & $\cdots$ & $<0.002$ & $<0.01$ \\
\hline DV96-3 & 0.04 & 0.004 & 0.08 & 1.02 & 0.004 & $<0.04$ & 0.15 & $<0.002$ & $<0.1$ & 0.58 & 0.91 & 0.02 & $<0.0001$ & 1.45 & 0.21 & $<0.002$ & --- & 0.013 & 0.01 \\
\hline DV96-4 & 0.034 & 0.004 & 0.06 & 2.9 & $<0.002$ & $<0.04$ & 4.01 & $<0.002$ & $<0.1$ & 0.57 & 0.53 & 0.12 & $<0.0001$ & 1.11 & 0.22 & $<0.002$ & --- & 0.012 & $<0.01$ \\
\hline DV96-5 & 0.043 & $<0.002$ & 0.06 & 0.95 & $<0.002$ & $<0.04$ & 0.08 & $<0.002$ & $<0.1$ & 0.61 & 0.78 & 0.094 & $<0.0001$ & 0.9 & 0.35 & $<0.002$ & --- & 0.012 & $<0.01$ \\
\hline DV96-6 & 0.053 & 0.003 & 0.07 & 0.91 & $<0.002$ & $<() .(94$ & 0.11 & $<0.002$ & $<0.1$ & 0.61 & 0.71 & 0.015 & $<0.0001$ & 0.76 & 0.31 & $<0.002$ & --- & 0.012 & $<0.01$ \\
\hline DV97-32 & --- & --- & --- & 19.1 & --- & $<0.02$ & 57.4 & -.. & $<0.05$ & --- & --- & --- & -- & --- & $<0.05$ & --- & --- & -- & --- \\
\hline DV97-33 & 0.056 & $<0.002$ & 0.059 & 0.82 & $<0.002$ & $<0.05$ & $<0.05$ & 0.002 & $<0.1$ & 0.73 & 8.91 & 0.046 & $<0.001$ & 1.76 & 0.27 & $<0.002$ & $<0.005$ & 0.012 & $<0.01$ \\
\hline
\end{tabular}




\section{Table 5: Continued}

Sample

DV97-34

DV97-35

DV97-36

DV97-37

DV97-40

DV97-41

DV97-42

DV98-97

DV98-98

DV98-143

DV98-161

DV98-162

DV98-163

DV99-198

DV99-205

DV99-206

DV99-207

DV99-208
$\begin{array}{llllllll}\mathbf{I} & \mathrm{Mn} & \mathrm{Mo} & \mathrm{NH}_{4} & \mathrm{Ni} & \mathrm{NO}_{2} & \mathrm{NO}_{3} & \mathrm{~Pb}\end{array}$

$\begin{array}{llllllll}0.044 & <0.002 & 0.07 & 3.22 & <0.002 & <0.05 & 7.08 & 0.002\end{array}$

$\begin{array}{llllllll}0.039 & <0.002 & 0.06 & 3.23 & <0.002 & <0.05 & 7.35 & 0.002\end{array}$

$\begin{array}{llllllll}0.051 & <0.002 & 0.07 & 0.76 & <0.002 & <0.05 & <0.05 & 0.002\end{array}$

$\begin{array}{llllllll}0.052 & <0.002 & 0.08 & 0.81 & <0.002 & <0.05 & <0.05 & 0.002\end{array}$

$\begin{array}{llllllll}0.055 & <0.002 & 0.1 & 0.73 & <0.002 & <0.05 & <0.05 & 0.002\end{array}$

$\begin{array}{llllll}-.- & - & - & 19.6 & \ldots & <0.02\end{array}$

$\begin{array}{cccccc}--- & -- & -- & 19.1 & --- & 0.03 \\ 0.04 & <0.002 & 0.06 & 0.94 & <0.002 & <0.05\end{array}$

$0.04<0.002 \quad 0.068 \quad 1.02<0.002<0.04$

$\begin{array}{cccccc}<0.01 & 0.008 & <0.002 & 15.8 & <0.002 & 10.2\end{array}$

$\begin{array}{llllll}0.05 & <0.002 & 0.074 & 0.99 & <0.002 & <0.05\end{array}$

$\begin{array}{llllll}0.01 & 0.14 & 0.015 & 7.59 & <0.002 & 5.35\end{array}$

$\begin{array}{llllll}0.02 & 0.16 & 0.006 & 7.88 & <0.002 & 1.48\end{array}$

$\begin{array}{llllll}0.05 & <0.002 & 0.1 & 0.81 & <0.002 & <0.05\end{array}$

$\begin{array}{llllll}0.04 & <0.002 & 0.09 & 0.81<0.002 & <0.05\end{array}$

$<0.01<0.002<0.002 \quad 16.5<0.002<0.01$

$\begin{array}{llllll}0.05 & <0.002 & 0.1 & 0.89 & <0.002 & <0.05\end{array}$
$0.034<0.002 \quad 0.041-1.51<0.002<0.05$
60.1

30.3

$<0.05<0.002$

$9.24<0.002$

$6.17<0.002$

$<0.05<0.002$

$0.07<0.002$

$15.2<0.002$

$0.29<0.002$
$<0.1 \quad 0.55$

$<0.1 \quad 0.62$

$<0.1 \quad 0.72$

$<0.1 \quad 0.72$

$<0.1 \quad 0.74$

$<0.05$

$<0.1$

$<0.1 \quad 0.5$

$<0.05 \quad 0.047$

$<0.1 \quad 0.63$

$\begin{array}{cc}<0.1 & 0.076 \\ <0.02 & 0.081\end{array}$

$<0.1 \quad 0.062$

$<0.1 \quad 0.69$

$<0.02 \quad 0.006$

Other Geothermal and On-Site Water Wells

DV96-1

DV97-38

DV97-39

DV97-53

DV97-54

DV97-55

DV97-59

DV97-67

DV98-96

DV98-99

DV98-100

DV98-102

DV98-103

DV98-104

DV98-111

DV98-122

DV98-123

Dixie Jack \#1

Dixie Jack \#4

Dixie Jack \#7

DV98-160

\section{$0.027 \quad 0.259 \quad 0.03$}

$0.72<0.002<0.02$

$\begin{array}{lllllll}0.037 & 0.28 & 0.01 & 0.56 & <0.002 & <0.02\end{array}$

$\begin{array}{lllllll}<0.005 & 0.006 & 0.002 & 13.1 & <0.002 & <0.02\end{array}$

$\begin{array}{lllllll}0.008 & 0.009 & 0.006 & 18.6 & 0.011 & <0.02\end{array}$

$\begin{array}{llllll}0.005 & 0.015 & 0.31 & 9.96 & <0.002 & <0.02\end{array}$

$\begin{array}{llllll}0.052 & 0.017 & 0.005 & 3.43 & <0.002 & 8.65\end{array}$

$\begin{array}{llllll}0.033 & 0.13 & <0.002 & 2.06 & <0.002 \quad<0.1\end{array}$

$\begin{array}{llllll}0.04 & 0.27 & <0.01 & 0.53 & <0.002<0.02\end{array}$

$\begin{array}{llllll}-- & -- & -- & 11.3 & -- & <0.02 \quad<0.02\end{array}$

$\begin{array}{lllllllll}-- & -- & -- & 12.9 & -- & 0.03 & 0.08 & -- & <0.05\end{array}$

$\begin{array}{lllllllll}-- & -- & -- & 14.7 & -- & <0.02 & <0.02 & -- & <0.05\end{array}$

$\begin{array}{lllllllll}0.03 & 0.12 & <0.01 & 2.08 & <0.002 & <0.05 & <0.05 & <0.002 & <0 .\end{array}$

$\begin{array}{lllllllll}0.02 & 0.007 & 0.01 & 2.02 & <0.002 & <0.05 & <0.05 & -- & <0.1\end{array}$

$\begin{array}{lllllllll}<0.01 & 0.38 & 0.02 & 0.76 & <0.002 & <0.05 & 0.62 & <0.002 & 2.43\end{array}$

$\begin{array}{ccccccccc}<0.01 & 0.44 & <0.01 & 0.04 & <0.002 & <0.02 & 0.03 & <0.002 & <0.05\end{array}$

$\begin{array}{llllllllll}0.03 & 0.14 & 0.045 & 1.04 & 0.003 & <0.1 & 0.53 & 0.003 & <0.05\end{array}$

$\begin{array}{lllllllll}0.03 & 0.009 & 0.009 & 1.83 & <0.002 & <0.1 & 0.25 & 0.004 & 1.56\end{array}$

$\begin{array}{lllllllll}0.01 & 0.002 & 0.008 & 0.44 & <0.002 & 0.05 & 0.86 & <0.002 & <0.05\end{array}$

$\begin{array}{lllllllll}0.03 & 0.27 & 0.025 & 0.48 & <0.002 & <0.02 & 0.35 & <0.002 & <0.05\end{array}$

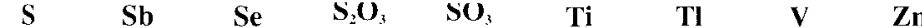

$\begin{array}{lllllllll}5.44 & 0.0084 & <0.001 & 0.84 & <0.05 & <0.002 & <0.005 & 0.01 & <0.01\end{array}$

$\begin{array}{lllllllll}5.64 & 0.0081 & <0.001 & 0.06 & <0.05 & <0.002 & <0.005 & 0.01 & 0.02\end{array}$

$\begin{array}{lllllllll}6.78 & 0.15 & <0.001 & <0.01 & 0.26 & <0.002 & <0.005 & 0.014 & 0.02\end{array}$

$\begin{array}{llllllllll}6.4 & 0.022 & <0.001 & <0.01 & 0.39 & <0.002 & <0.005 & 0.013 & <0.01\end{array}$

$\begin{array}{lllllllll}7.8 & 0.026 & <0.001 & 1.62 & 0.42 & <0.002 & <0.005 & 0.011 & <0.01\end{array}$

$\begin{array}{ccccccccc}--- & --- & --- & -- & <0.05 & -- & --- & --- & -- \\ 19.9 & 0.055 & <0.001 & 0.25 & 0.49 & <0.002 & <0.005 & 0.013 & <0.01\end{array}$

$\begin{array}{ccccccccc}-- & -- & -- & -- & <0.05 & --- & -- & --- & --- \\ 0.18 & -. & --- & 1.58 & <0.05 & <0.002 & -- & 0.012 & <0.01\end{array}$

0.28

$\begin{array}{lllllllll}0.01 & -- & -- & <0.01 & <0.02 & <0.002 & --- & <0.002 & <0.01\end{array}$

$\begin{array}{lllllllll}0.15 & -- & -- & 1.25 & <0.1 & <0.002 & -- & 0.012<0.01\end{array}$

$\begin{array}{lllllllll}<0.01 & -- & \cdots & <0.01 & <0.1 & <0.002 & -- & 0.006 & 0.03\end{array}$

$\begin{array}{lllllllll}0.37 & -- & -- & <0.01 & <0.02 & <0.002 & --- & 0.012 & 0.03\end{array}$

$\begin{array}{llllllll}--- & 1.57 & <0.1 & <0.002 & -- & 0.013<0.01\end{array}$

$\begin{array}{llll}--- & 1.59 & <0.1<0.002\end{array}$

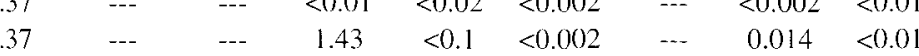

$<0.0002<0.0001<0.01$

$<0.0002<0.001<0.0$

$0.003<0.001 \quad 3.68$

$0.012<0.001 \quad 3.35$

$0.012<0.001 \quad 1.77$

$<0.001<0.001<0.01$

$<0.001<0.001 \quad 1.57$

$---<0.01$

$<0.1<0.002$

$<0.002 \quad 0.01$

$<0.05 \quad 0.009<0.005 \quad 0.019<0.01$

$0.31<0.002 \quad--<0.002<0.01$

$<0.05 \quad 0.003 \quad--<<0.002<0.01$

$<0.05<0.002 \quad---<0.002<0.01$

$<0.05<0.002 \quad--<0.002<0.01$

$<0.1<0.002 \quad--\quad<0.002 \quad 0.01$

$<0.05<0.002 \quad---\quad 0.018<0.01$

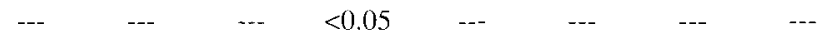

$\begin{array}{llllllll}-- & -- & -- & 0.66 & --- & -- & -- & --\end{array}$

$<0.05$

$<0.05<0.002 \quad--<<0.002<0.01$

$<0.05 \quad<0.002 \quad--\quad<0.002<0.01$

$<0.05<0.002 \quad--\quad<0.002<0.01$

$\begin{array}{llllll}<0.05 & <0.002 & -- & 0.025 & 0.02\end{array}$

$\begin{array}{llll}<.05 & <0.002 \quad-- & <0.002<0.01\end{array}$

$\begin{array}{lllll}<0.05 & <0.002<0.002 & 0.009 & <0.01\end{array}$

$\begin{array}{lllll}<0.05 & <0.002 & <0.002 & 0.003 & 0.01\end{array}$

$<0.05<0.002<0.002<0.002<0.01$

$<0.05<0.002 \quad--\quad 0.021<0.01$ 
Table 5: Continued

Sample

98-168

DV98-175

DV99-181

Background Springs

DV97-46

DV97-47

DV97-48

DV97-50

DV $97-51 \mathrm{~b}$

DV97-52

DV97-56

DV97-60

DV97-61

DV97-62

DV97-64

DV97-65

DV97-66

$\pm \quad$ DV97-68

DV97-69

DV97-72

DV98-106

DV98-112

DV98-113

DV98-114

DV98-117

DV98-118

DV98-120

DV98-128

DV98-129

DV98-131

DV98-132

DV98-169

DV98-170

DV98-176

DV98-177

DV98-178

DV98-179

DV99-209

DV99-210

DV99-211
$\begin{array}{lllllllll}\mathrm{I} & \mathrm{Mn} & \mathrm{Mo} & \mathrm{NH}_{4} & \mathrm{Ni} & \mathrm{NO}_{2} & \mathrm{NO}_{3} & \mathrm{~Pb} & \mathrm{PO}_{4}\end{array}$

$\begin{array}{lllllllll}0.02 & 0.035 & <0.002 & 1.95 & <0.002 & <0.05 & <0.05 & 0.002<0.1\end{array}$

$\begin{array}{lllllllll}0.03 & 0.26 & 0.015 & 0.52 & <0.002 & 1.77 & 0.19 & <0.002<0.1\end{array}$

$\begin{array}{llllllllll}0.012 & 0.045 & <0.002 & 0.11 & <0.002 & <0.02 & 2.32 & 0.002 & <0.05 & 0.13\end{array}$

$\begin{array}{llllllllll}0.007 & 0.05 & <0.002 & 0.69 & <0.002 & 0.6 & <0.02 & 0.002 & <0.05 & 0.12\end{array}$

$\begin{array}{llllllllll}0.012 & 0.016 & <0.002 & 1.98 & <0.002 & 0.21 & <0.02 & <0.002 & <0.05 & 0.17\end{array}$

$\begin{array}{llllllllll}<0.005 & 0.007 & 0.004 & 0.1 & <0.002 & <0.02 & 0.72 & <0.002 & <0.05 & 0.002\end{array}$

$\begin{array}{llllllllll}<0.005 & 0.002 & 0.009 & 0.05 & <0.002 & <0.02 & <0.02 & <0.002 & <0.05 & 0.024\end{array}$

$\begin{array}{llllllllll}<0.005 & 0.006 & 0.007 & 0.05 & 0.025 & <0.02 & 1.77 & <0.002 & 0.11 & 0.013\end{array}$

$\begin{array}{llllllllll}<0.005 & <0.002 & 0.004 & 0.08 & <0.002 & <0.05 & <0.05 & <0.002 & <0.1 & 0.43\end{array}$

$\begin{array}{llllllllll}<0.005 & 0.002 & <0.002 & 0.05 & <0.002 & <0.02 & <0.02 & <0.002 & <0.05 & 0.057\end{array}$

$\begin{array}{lllllllll}<0.005 & <0.002<0.002 & 0.05 & <0.002 & <0.02 & 0.48 & <0.002 & <0.05 & 0.058\end{array}$

$\begin{array}{llllllllll}<0.005 & <0.002 & 0.007 & 0.05 & <0.002 & <0.05 & 0.34 & <0.002 & <0.05 & 0.035\end{array}$

$\begin{array}{lllllllllll}<0.005 & 0.007 & 0.003 & 0.25 & <0.002 & <0.05 & <0.02 & <0.002 & <0.05 & 0.006\end{array}$

$\begin{array}{lllllllll}<0.005 & <0.002 & 0.01 & 0.18 & <0.002 & <0.05 & <0.02 & <0.002<0.05 & <0.002\end{array}$

$\begin{array}{llllllllll}<0.005 & <0.002 & 0.007 & 0.18 & <0.002 & <0.05 & <0.02 & <0.002 & <0.05 & <0.002\end{array}$

$\begin{array}{llllllllll}<0.005 & <0.002<0.002 & 0.21 & <0.002 & <0.05 & 5.38 & <0.002 & <0.05 & 0.009\end{array}$

$\begin{array}{llllllllllll}<0.005 & 0.003 & <0.002 & 0.14 & <0.002 & <0.1 & <0.05 & <0.002 & <0.1 & 0.004\end{array}$

$\begin{array}{llllllllll}0.013 & 0.006 & <0.002 & 0.2 & <0.002 & <0.05 & 0.07 & <0.002 & <0.05 & 0.048\end{array}$

$\begin{array}{lllllllll}<0.005 & <0.002<0.002 & 0.16 & <0.002 & <0.02 & <0.02 & <0.002 & <0.05 & <0.002\end{array}$

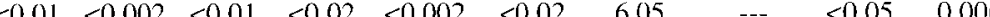

$\begin{array}{llllllllll}<0.01 & 0.015 & <0.01 & 1.97 & <0.002 & <0.02 & <0.02 & --- & <0.05 & 0.16\end{array}$

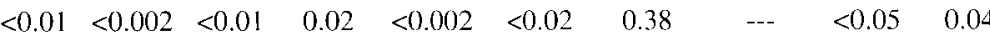

$<0.01<0.002<0.01<0.02<0.002<0.02 \quad 0.20 \quad---<<0.05 \quad 0.030$

$\begin{array}{llllllllll}<0.01 & 0.041 & <0.01 & 0.51 & <0.002 & <0.02 & 0.11 & \ldots & <0.05 & 0.12\end{array}$

$<0.01 \quad 0.004 \quad<0.01 \quad<0.02 \quad<0.002 \quad<0.05 \quad<0.05 \quad<0.002 \quad<0.1 \quad 0.006$

$\begin{array}{llllllllll}<0.01 & 0.009 & 0.03 & 0.21 & <0.002 & <0.02 & <0.02 & <0.002 & <0.05 & 0.056\end{array}$

$\begin{array}{llllllllll}<0.01 & 0.12 & <0.01 & 1.55 & <0.002 & <0.02 & <0.02 & <0.002<0.0 .5 & 0.27\end{array}$

$<0.01 \quad 0.017 \quad<0.01 \quad 0.04 \quad<0.002 \quad<0.02 \quad<0.02 \quad<0.002 \quad<0.05 \quad 0.22$

$\begin{array}{llllllllll}<0.01 & 0.002 & 0.04 & <0.02 & <0.002 & <0.05 & 0.13 & <0.002 & <0.1 & 0.005\end{array}$

$\begin{array}{llllll}<0.01<0.002 & 0.03<0.02<0.002<0.02 & <0.02<0.002<0.05<0.002\end{array}$

$\begin{array}{llllllllll}<0.01 & <0.002<0.002 & 0.05 & <0.002 & <0.02 & <0.02 & <0.002 & <0.05 & <0.002\end{array}$

$\begin{array}{lllllllll}<0.01 & <0.002<0.002 & 0.03 & <0.002 & <0.02 & <0 .(02 & 0.003 & 0.15 & <0.002\end{array}$

$\begin{array}{lllllllll}<0.01<0.002<0.002 & 0.03 & <0.002 & <0.02 & 2.38 & <0.002 & <0.05 & 0.002\end{array}$

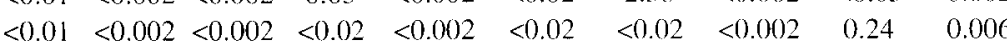

$<0.01<0.002<0.002<0.02<0.002<0.02 \quad 1.53<0.002<0.05 \quad 0.004$

$<0.01<0.002<0.002 \quad 0.02<0.002<0.02 \quad 4.78<0.002<0.05<0.002$

$\begin{array}{llllllllll}<0.01 & 0.002 & 0.03 & 0.08 & <0.002 & <0.05 & <0.01 & 0.002 & <0.02 & 0.45\end{array}$

$\begin{array}{cccccccccc}<0.01 & 0.22 & <0.002 & 0.07 & <0.002 & <0.05 & <0.01 & 0.003 & <0.02 & 0.56 \\ <0.01 & <0.002 & <0.002 & <0.02 & <0.002 & <0.01 & 0.04 & <0.002 & <0.02 & 0.06\end{array}$

$<0.01<0.002<0.002<0.02<0.002<0.01$

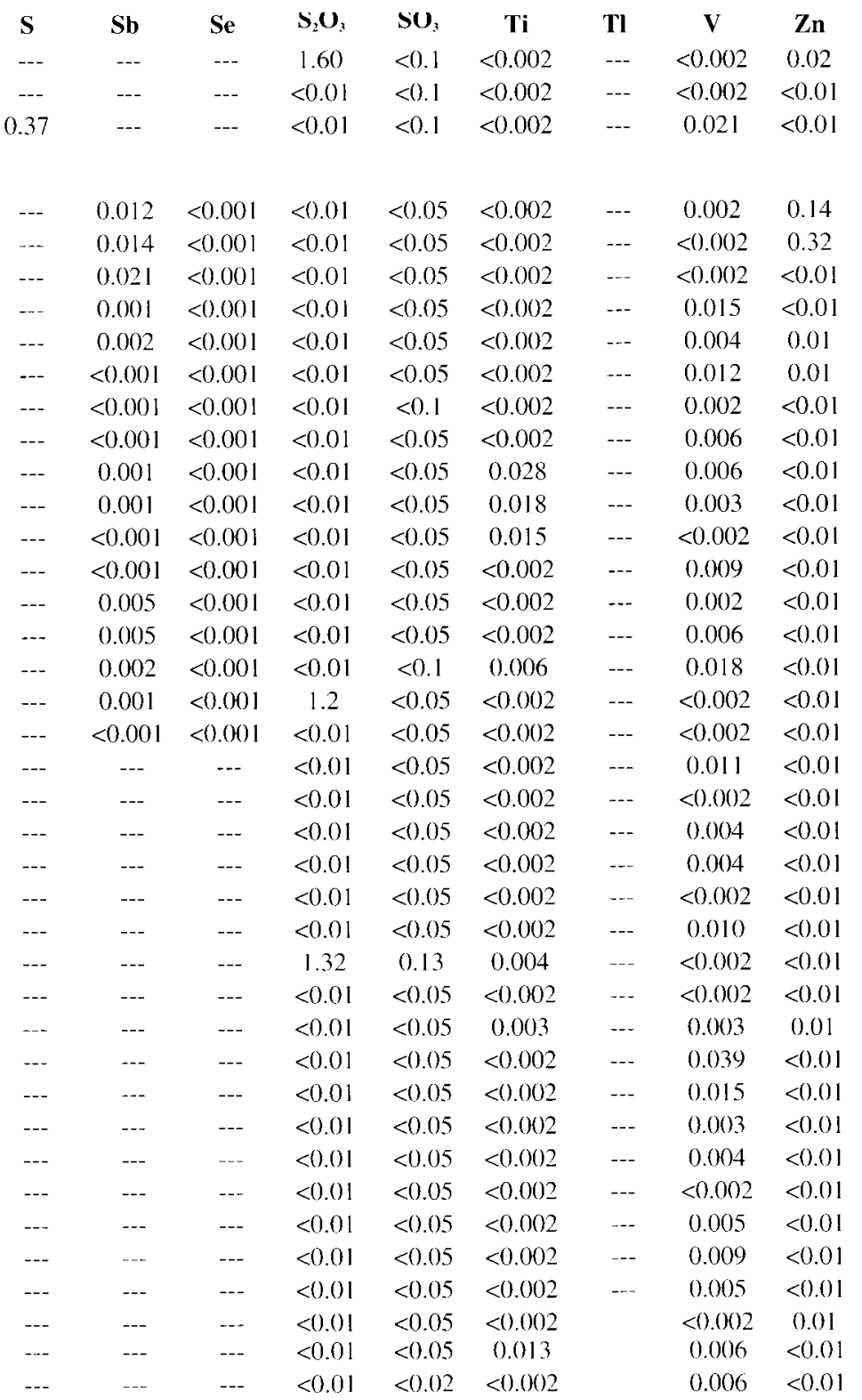




\section{Table 5: Continued}

\begin{tabular}{|c|c|c|c|c|c|c|c|c|c|c|c|c|c|c|c|c|c|c|c|}
\hline $\begin{array}{l}\text { Sample } \\
\text { Backgroun }\end{array}$ & I & Mn & Mo & $\mathbf{N H}_{4}$ & $\mathrm{Ni}$ & $\mathrm{NO}_{2}$ & $\mathrm{NO}_{3}$ & \multicolumn{5}{|c|}{ Background Wells } & Se & $\mathbf{S}_{2} \mathbf{U}_{3}$ & $\mathrm{SO}_{3}$ & $\mathbf{T i}$ & Tl & V & $\mathrm{Zn}$ \\
\hline DV $97-49$ & 0.023 & 0.03 & $<0.002$ & 0.09 & $<0.002$ & $<0.02$ & 0.05 & $<0.002$ & $<0.05$ & 0.019 & --- & $<0.001$ & $<0.001$ & $<0.01$ & $<0.05$ & $<0.002$ & --- & $<0.002$ & 0.23 \\
\hline DV97-57 & $<0.005$ & 0.007 & $<0.002$ & 0.11 & $<0.002$ & $<0.05$ & $<0.02$ & $<0.002$ & $<0.05$ & 0.026 & --- & 0.002 & $<0.001$ & $<0.01$ & $<0.05$ & $<0.002$ & --- & $<0.002$ & $<0.01$ \\
\hline DV97-70 & $<0.005$ & $<0.002$ & 0.023 & 0.06 & $<0.002$ & $<0.02$ & 0.81 & $<0.002$ & $<0.05$ & 0.002 & $\cdots$ & 0.001 & $<0.001$ & $<0.01$ & $<0.05$ & $<0.002$ & --- & 0.02 & $<0.01$ \\
\hline DV97-71 & $<0.005$ & $<0.002$ & 0.018 & 0.04 & $<0.002$ & $<0.02$ & 0.31 & 0.004 & $<0.05$ & 0.002 & --- & $<0.001$ & $<0.001$ & $<0.01$ & $<0.05$ & $<0.002$ & --- & 0.015 & $<0.01$ \\
\hline DV98-115 & $<0.01$ & $<0.002$ & $<0.01$ & $<0.02$ & $<0.002$ & $<0.02$ & 7.27 & --- & $<0.05$ & 0.055 & --- & --- & --- & $<0.01$ & $<0.05$ & $<0.002$ & -.. & 0.013 & 0.01 \\
\hline DV98-116 & $<0.01$ & $<0.002$ & 0.02 & $<0.02$ & $<0.002$ & $<0.05$ & 36.0 & --- & $<0.1$ & 0.005 & --- & --- & & $<0.01$ & $<0.05$ & $<0.002$ & -.- & 0.007 & 0.10 \\
\hline DV98-172 & $<0.01$ & 0.34 & $<0.002$ & 0.06 & $<0.002$ & $<0.02$ & $<0.02$ & $<0.002$ & $<0.05$ & $<0.002$ & --- & --- & --- & $<0.01$ & $<0.05$ & $<0.002$ & --- & $<0.002$ & $<0.01$ \\
\hline \multicolumn{20}{|c|}{ Background Streams/Rain } \\
\hline DV97-58 & $<0.005$ & $<0.002$ & 0.005 & 0.31 & $<0.002$ & $<0.05$ & $<0.02$ & $<0.002$ & $<0.05$ & 0.005 & --- & $<0.001$ & $<0.001$ & $<0.01$ & $<0.05$ & $<0.002$ & --- & 0.005 & $<0.01$ \\
\hline DV98-107 & $<0.01$ & $<0.002$ & 0.02 & $<0.02$ & $<0.002$ & $<0.02$ & 0.78 & --- & $<0.05$ & 0.005 & --- & --- & --- & $<0.01$ & $<0.05$ & $<0.002$ & --- & 0.009 & $<0.01$ \\
\hline DV98-110 & $<0.01$ & 0.002 & 0.02 & $<0.02$ & $<0.002$ & $<0.02$ & $<0.02$ & --- & $<0.05$ & 0.005 & --- & $--\cdot$ & --- & $<0.01$ & $<0.05$ & 0.004 & --- & 0.009 & $<0.01$ \\
\hline DV98-119 & $<0.01$ & 0.012 & $<0.01$ & $<0.02$ & $<0.002$ & $<0.02$ & 3.30 & $<0.002$ & $<0.05$ & 0.004 & --- & --- & --- & $<0.01$ & $<0.05$ & 0.011 & --- & $<0.002$ & $<0.01$ \\
\hline DV98-121 & $<0.01$ & 0.003 & 0.02 & $<0.02$ & $<0.002$ & $<0.02$ & $<0.02$ & $<0.002$ & $<0.05$ & 0.002 & $\cdots$ & --- & --- & $<0.01$ & $<0.05$ & $<0.002$ & --- & 0.004 & $<0.01$ \\
\hline DV98-125 & $<0.01$ & $<0.002$ & 0.04 & 0.22 & $<0.002$ & $<0.02$ & $<0.02$ & $<0.002$ & $<0.05$ & 0.011 & --- & --- & --- & $<0.01$ & $<0.05$ & $<0.002$ & --- & $<0.002$ & 0.05 \\
\hline DV98-126 & $<0.01$ & 0.019 & 0.02 & 0.04 & $<0.002$ & $<0.02$ & $<0.02$ & $<0.002$ & $<0.05$ & 0.004 & --- & --- & --- & $<0.01$ & $<0.05$ & 0.012 & --- & 0.002 & $<0.01$ \\
\hline DV98-127 & $<0.01$ & 0.007 & 0.02 & 0.02 & $<0.002$ & $<0.02$ & $<0.02$ & $<0.002$ & 0.18 & 0.005 & --- & --- & $\cdots$ & $<0.01$ & $<0.05$ & 0.021 & --- & $<0.002$ & $<0.01$ \\
\hline DV98-130 & $<0.01$ & 0.003 & $<0.01$ & $<0.02$ & $<0.002$ & $<0.02$ & $<0.02$ & $<0.002$ & $<0.05$ & 0.004 & --- & --- & --- & $<0.01$ & $<0.05$ & $<0.002$ & -- & $<0.002$ & $<0.01$ \\
\hline DV98-171 & $<0.01$ & $<0.002$ & $<0.002$ & 0.02 & $<0.002$ & $<0.02$ & $<0.02$ & $<0.002$ & $<0.05$ & $<0.002$ & -- & --- & --- & $<0.01$ & $<0.05$ & $<0.002$ & $\ldots$ & 0.002 & $<0.01$ \\
\hline DV98-173 & $<0.01$ & 0.002 & 0.003 & $<0.02$ & $<0.002$ & $<0.02$ & $<0.02$ & $<0.002$ & $<0.05$ & 0.003 & --- & --- & --- & $<0.01$ & $<0.05$ & $<0.002$ & --- & 0.004 & $<0.01$ \\
\hline DV98-174 & $<0.01$ & $<0.002$ & $<0.002$ & $<0.02$ & $<0.002$ & $<0.05$ & $<0.05$ & 0.002 & $<0.1$ & 0.002 & --- & --- & --- & $<0.01$ & $<0.1$ & $<0.002$ & --- & 0.003 & $<0.01$ \\
\hline DV98-180 & $<0.01$ & $<0.002$ & $<0.002$ & 0.02 & $<0.002$ & $<0.02$ & 0.68 & $<0.002$ & 0.12 & $<0.002$ & --- & --- & --- & $<0.01$ & $<0.05$ & $<0.002$ & --- & 0.006 & $<0.01$ \\
\hline DV99-2I3 & 0.19 & 0.022 & 0.003 & 0.16 & $<0.002$ & $<0.5$ & $<0.5$ & 0.003 & $<1$ & 0.035 & --- & --- & --- & $<0.01$ & $<1$ & $<0.002$ & --- & 0.006 & 0.01 \\
\hline \multicolumn{20}{|c|}{ Fumarole Condensates } \\
\hline DV97-43 & --- & 0.045 & --- & --- & --- & 0.02 & $<0.04$ & --- & $<0.05$ & --- & --- & --- & -- & --- & $<0.05$ & --- & --- & $<0.002$ & $<0.01$ \\
\hline DV97-44 & --- & 0.031 & $<0.002$ & --- & 0.002 & 0.03 & 0.48 & $<0.002$ & $<0.05$ & 0.01 & --- & -- & --- & -- & $<0.05$ & --- & --- & 0.01 & 0.1 \\
\hline DV98-108 & --- & --- & --- & 21.5 & --- & $<0.02$ & $<0.02$ & --- & $<0.05$ & --- & --- & --- & --- & --- & 0.11 & --- & --- & --- & --- \\
\hline DV98-I64 & --- & --- & --- & 21.1 & --- & $<0.02$ & 0.06 & --- & $<0.05$ & $\cdots$ & -- & --- & --- & --- & $<0.02$ & --- & -- & --- & --- \\
\hline DV98-109 & --- & -- & --- & 58.1 & --- & $<0.02$ & $<0.02$ & --- & $<0.05$ & $\cdots$ & --- & --- & --- & --- & $<0.05$ & --- & --- & -- & $\cdots$ \\
\hline DV98-165 & --- & --- & --- & 56.0 & --- & $<0.02$ & 0.02 & --- & $<0.05$ & --- & --- & $\ldots$ & --- & --- & $<0.02$ & --- & --- & --- & --- \\
\hline DV98-166 & --- & --- & --- & 0.17 & --- & $<0.02$ & 0.06 & --- & $<0.05$ & --- & --- & --- & --- & --- & $<0.02$ & --- & --- & --- & --- \\
\hline
\end{tabular}


Table 6: Analytical Results From Various Aluminum Samples And Extractions (All values in ppm).

Sample
Brines
DIXE102-W
DV96-8
DV96-9
DV97-11
DV97-13
DV97-14
DV97-16
DV97-18
DV97-20
DV97-23
DV97-25
DV97-26
DV97-29
DV97-30
DV98-73
DV98-75
DV98-77
DV98-79
DV98-80
DV98-82
DV98-84
DV98-86
DV98-88
DV98-90
DV98-92
DV98-95
DV98-133
DV98-135
DV98-138
DV98-140
DV98-141
DV98-145
DV98-147
DV98-148
DV98-150
DV98-152
DV98-154
DV98-156
DV98-159
DV99-182
DV99-184
DV99-186
DV99-188
DV99-190
DV99-194
DV99-196
DV99-197
DV99-199

Name or Description

V $102+$ V 103 Separator

76-7 Well

V101 Separator

73-7 Well

84-7 Well

74-7 Well

V102 + V 103 Separator

V105 Separator

82A-7 Well

73B-7 Well

27-33 Well

V101 Separator

37-33 Well

28-33 Well

V101 Separator

27-33 Well

37-33 Well

28-33 Well

76A-7 Well

V102 + V103 Separator

74-7 Well

63-7 Well

73-7 Well

82A-7 Well

V105 Separator

73B-7 Well

27-33 Well

27-33 Well

V101 Separator

37-33 Well

28-33 Well

76A-7 Well

63-7 Well

V $102+$ V 103 Separator

74-7 Well

73-7 Well

73B-7 Well

82A-7 Well

V105 Separator

76A-7 Well

74-7 Well

V102 + V103 Separator

63-7 Well

73-7 Well

V105 Separator

82A-7 Well

73B-7 Well

37-33 Well
Date

$10 / 02 / 95$

$10 / 25 / 96$

$10 / 25 / 96$

$10 / 29 / 97$

$10 / 29 / 97$

$10 / 29 / 97$

$10 / 29 / 97$

$10 / 29 / 97$

$10 / 29 / 97$

10/30/97

10/30/97

$10 / 30 / 97$

10/30/97

10/30/97

$04 / 28 / 98$

$04 / 28 / 98$

$04 / 28 / 98$

$04 / 28 / 98$

$04 / 28 / 98$

$04 / 28 / 98$

$04 / 28 / 98$

$04 / 28 / 98$

$04 / 29 / 98$

04/29/98

$04 / 29 / 98$

04/29/98

10/20/98

10/20/98

$10 / 21 / 98$

$10 / 21 / 98$

$10 / 21 / 98$

$10 / 22 / 98$

$10 / 22 / 98$

$10 / 22 / 98$

$10 / 22 / 98$

$10 / 22 / 98$

$10 / 22 / 98$

$10 / 23 / 98$

$10 / 23 / 98$

$05 / 04 / 99$

05/04/99

$05 / 04 / 99$

05/04/99

05/04/99

05/05/99

05/05/99

05/05/99

05/05/99

\section{Total Al \\ Ionized $\mathrm{Al}^{\mathrm{b}}$}

(0.45 micron)

Ionized $\mathrm{Al}^{\mathrm{b}}$

(0.45 micron)

Ionized $\mathbf{A l}^{\mathrm{C}}$

1.41
1.12
1.54
1.04
1.12
1.13
1.12
1.05
1.02

---
0.050
0.101
0.063
0.063
0.082
0.100

0.126

0.043

1.08

1.44

1.47

0.99

1.27

1.34

1.29

1.42

1.39

1.04

1.08

1.09

1.03

1.04

1.01

1.01

0.97

1.31

0.39

1.32

1.04

1.36

1.00

1.02

1.01

1.02

1.02

0.81

0.90

0.99

0.89

0.97

0.97

0.98

1.00

0.95

0.86

0.96

1.37

\subsection{9}

0.043

0.055

0.024

0.060

$$
0.022
$$

0.028

0.022

0.032

0.035

0.027

0.039

0.022

0.057

0.054

0.036

0.042

0.032

0.001

0.041

0.051

0.044

0.022

0.025

0.038

0.029

0.026

0.037

0.061

0.043

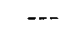

$$
\text { --- }
$$$$
\cdots
$$$$
\cdots
$$$$
-
$$$$
\cdots
$$$$
\cdots
$$

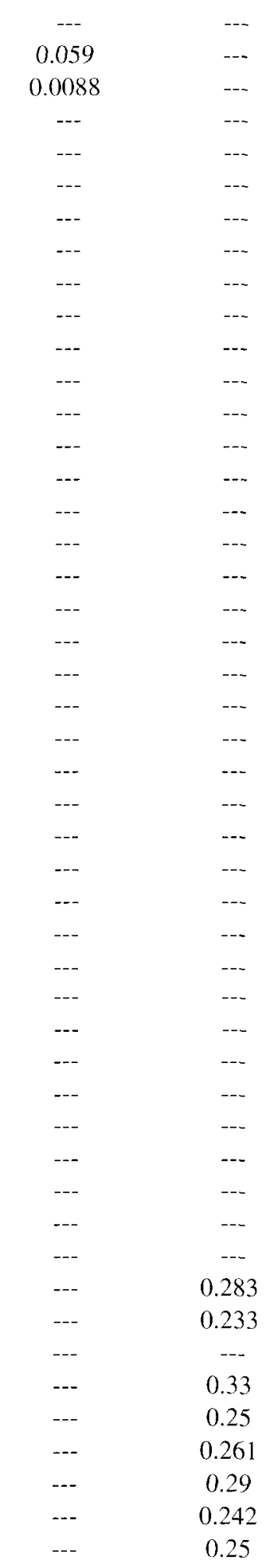


Table 6: Continued

$\begin{array}{ll}\text { Sample } & \text { Name or Description } \\ \text { DV99-200 } & \text { 28-33 Well } \\ \text { DV99-204 } & \text { V101 Separator } \\ \text { DV74782786-brine 2 } & \text { 74-7 Well Archived } \\ \text { DV76781986-brine 4 } & \text { 76-7 Well Archived } \\ \text { DV453382186-brine 6 } & \text { 45-33 Well Archived } \\ \text { DV73782886-brine 8 } & \text { 73-7 Well Archived } \\ \text { DV321882686-brine 10 } & \text { 32-18 Well Archived } \\ \text { DV651882686-brine 12 } & \text { 65-18 Well Archived } \\ \text { No number } & \text { 28-33 Well Archived }\end{array}$

\section{Injection Well/Power Plant Fluids}

$\begin{array}{ll}\text { DV96-2 } & \text { Condensate from plant } \\ \text { DV96-3 } & \text { LP Brine @ Plant } \\ \text { DV96-4 } & \text { 45-5 Injection Well } \\ \text { DV96-5 } & \text { Lamb I Injection Well } \\ \text { DV96-6 } & 65-18 \text { Injection Well } \\ \text { DV97-33 } & \text { LP Brine } \\ \text { DV97-34 } & 25-5+45-5 \text { Injectate } \\ \text { DV97-35 } & 25-5+45-5 \text { Injectate } \\ \text { DV97-36 } & 65-18 \text { Injection Well } \\ \text { DV97-37 } & 32-18 \text { Injection Well } \\ \text { DV97-40 } & \text { LP Brine @ Plant } \\ \text { DV97-42 } & \text { High P Brine @ plant } \\ \text { DV98-98 } & \text { LP Brine @ Plant } \\ \text { DV98-143 } & 25-5 \text { Injection Well } \\ \text { DV98-161 } & \text { Condensate from plant } \\ \text { DV98-162 } & \text { LP Brine@ Plant } \\ \text { DV98-163 } & 65-18 \text { Injection Well } \\ \text { DV99-198 } & 65-18 \text { Injection Well } \\ \text { DV99-205 } & \text { 25-5 + 45-5 Injectate } \\ \text { DV99-206 } & \text { LP Brine@ Plant } \\ \text { DV99-207 } & \text { Condensate from plant } \\ \text { DV99-208 } & 52-18+41-18 \text { Injectate }\end{array}$

Other Geothermal and On-Site Water Wells

$\begin{array}{lll}\text { DV96-1 } & \text { Domestic Well } & 10 / 24 / 96 \\ \text { DV97-38 } & \text { Domestic Well } & 10 / 31 / 97 \\ \text { DV97-39 } & \text { Goerenger Well } & 10 / 31 / 97 \\ \text { DV97-53 } & 46-32 \text { Well } & 11 / 05 / 97 \\ \text { DV97-54 } & 27-32 \text { Well } & 11 / 05 / 97 \\ \text { DV97-55 } & \text { 27-32 Well } & 11 / 05 / 97 \\ \text { DV97-59 } & 45-W-5 \text { Well } & 11 / 05 / 97 \\ \text { DV97-67 } & 66-21 \text { Well } & 11 / 07 / 97 \\ \text { DV98-96 } & \text { Goerenger Well } & 04 / 29 / 98 \\ \text { DV98-103 } & 45-14 \text { Well } & 04 / 30 / 98 \\ \text { DV98-104 } & 66-21 \text { Well } & 04 / 30 / 98 \\ \text { DV98-111 } & 62-21 \text { Well } & 05 / 01 / 98 \\ \text { DV98-122 } & 97-2 \text { Well } & 05 / 05 / 98 \\ \text { DV98-123 } & 32-6 \text { Well } & 05 / 06 / 98\end{array}$

$\begin{array}{cccc}\begin{array}{c}\text { Total Al } \\ (\mathbf{0 . 4 5} \text { micron })\end{array} & \begin{array}{c}\text { Ionized Al } \\ (\mathbf{0 . 2} \text { micron })\end{array} & \begin{array}{c}\text { Ionized Al } \\ (\mathbf{0 . 4 5} \text { micron })\end{array} & \begin{array}{c}\text { Ionized } \mathbf{A l}^{\mathbf{c}} \\ \text { (unfiltered) }\end{array} \\ 1.37 & --- & -- & 0.20 \\ 1.30 & --- & --- & 0.33 \\ 1.10 & --- & --- & --- \\ 1.19 & --- & --- & --- \\ 1.52 & --- & --- & --- \\ 0.99 & --- & --- & --- \\ 0.75 & --- & --- & --- \\ 0.69 & --- & --- & --- \\ 0.88 & --- & --- & ---\end{array}$

$\begin{array}{lcccc}10 / 24 / 96 & 0.14 & 0.0096 & 0.0106 & --- \\ 10 / 24 / 96 & 1.27 & 0.025 & 0.011 & --- \\ 10 / 24 / 96 & 1.21 & 0.0079 & 0.0087 & --- \\ 10 / 24 / 96 & 1.39 & 0.0004 & 0.0004 & --- \\ 10 / 24 / 96 & 1.35 & 0.0004 & 0.0014 & --- \\ 10 / 31 / 97 & 1.36 & 0.04 & --- & --- \\ 10 / 31 / 97 & 1.14 & 0.045 & --- & --- \\ 10 / 31 / 97 & 1.14 & 0.103 & --- & --- \\ 10 / 31 / 97 & 1.32 & 0.0021 & --- & --- \\ 10 / 31 / 97 & 1.34 & 0.0036 & --- & --- \\ 10 / 31 / 97 & 1.36 & 0.0081 & --- & --- \\ 10 / 31 / 97 & 1.38 & 0.038 & --- & --- \\ 04 / 29 / 98 & 1.22 & 0.024 & --- & --- \\ 10 / 21 / 98 & 1.19 & 0.023 & --- & --- \\ 10 / 23 / 98 & 0.07 & --- & --- & --- \\ 10 / 23 / 98 & 1.22 & 0.040 & --- & --- \\ 10 / 23 / 98 & <0.02 & 0.009 & 0.036 & --- \\ 05 / 05 / 99 & <0.02 & --- & --- & 0.001 \\ 05 / 06 / 99 & 1.19 & --- & --- & 0.549 \\ 05 / 06 / 99 & 1.26 & --- & --- & 0.774 \\ 05 / 06 / 99 & 0.04 & --- & --- & 0.004 \\ 05 / 06 / 99 & 1.32 & --- & --- & 0.319\end{array}$


Table 6: Continued

Sample

Dixie Jack \#1

Dixie Jack \#4

Dixie Jack \#7

DV98-160

DV98-168

DV98- 175

DV99-181

Name or Description
Gradient Well DJ \#1
Gradient Well DJ \#4
Gradient Well DJ \#7
Goerenger Well
38-32 Well
62-21 Well
Goerenger Well

\begin{tabular}{|c|c|c|c|c|}
\hline Date & $\begin{array}{c}\text { Total } A I^{a} \\
\text { (0.45 micron) }\end{array}$ & $\begin{array}{l}\text { Ionized } \mathrm{Al}^{\mathrm{b}} \\
\text { (0.2 micron) }\end{array}$ & $\begin{array}{c}\text { Ionized } \mathrm{Al}^{\mathrm{b}} \\
\text { (0.45 micron) }\end{array}$ & $\begin{array}{l}\text { Ionized } \mathbf{A} \mathbf{I}^{\mathrm{c}} \\
\text { (unfiltered) }\end{array}$ \\
\hline $05 / 17 / 98$ & $<0.02$ & -- & -- & --- \\
\hline $05 / 20 / 98$ & 0.07 & --- & --- & --- \\
\hline $05 / 14 / 98$ & 0.03 & -.- & --- & -.- \\
\hline $10 / 23 / 98$ & $<0.02$ & 0.001 & --- & --- \\
\hline $10 / 26 / 98$ & $<0.02$ & --- & --- & --- \\
\hline $10 / 28 / 98$ & $<0.02$ & --- & --- & --- \\
\hline 05/04/99 & $<0.02$ & -- & --- & 0.004 \\
\hline
\end{tabular}

"Analyses by ICP on filtered samples acidified with spectrographcially pure $\mathrm{HNO}_{3}$ (see Table 3).

${ }^{\mathrm{b}}$ Analyses by GFAA on filtered samples using MIBK extraction method of Barnes (1975).

"Analyses by GFAA on filtered samples using MIBK extraction method of Barnes (1975). Data were corrected using a "spike" procedure with duplicate unfiltered samples in which $1 \mathrm{ppm} \mathrm{Al}$ is added to a sample and the other is corrected. 
Table 7: Miscellaneous Isotope Data for Various Geothermal and Regional Fluids from the Dixie Valley Region, Nevada."

\begin{tabular}{|c|c|}
\hline Sample & Name or Description \\
\hline \multicolumn{2}{|l|}{ Brines } \\
\hline DV96-8 & 76-7 Well \\
\hline DV96-8b & 76-7 Well \\
\hline DV96-9 & VI01 Separator \\
\hline DV96-9b & V101 Separator \\
\hline DV97-11 & 73-7 Well \\
\hline DV97-13 & 84-7 Well \\
\hline DV97-14 & 74-7 Well \\
\hline DV97-16 & V $102+$ V103 Separator \\
\hline DV97-18 & V105 Separator \\
\hline DV97-20 & 82A-7 Well \\
\hline DV97-23 & 73B-7 Well \\
\hline DV97-25 & 27-33 Well \\
\hline DV97-26 & V101 Separator \\
\hline DV97-29 & 37-33 Well \\
\hline DV97-30 & 28-33 Well \\
\hline DV98-73 & V101 Separator \\
\hline DV98-75 & 27-33 Well \\
\hline DV98-77 & $37-33$ Well \\
\hline DV98-79 & 28-33 Well \\
\hline DV98-80 & 76A-7 Well \\
\hline DV98-82 & V102+V 103 Separator \\
\hline DV98-84 & 74-7 Well \\
\hline DV98-86 & 63-7 Well \\
\hline DV98-88 & 73-7 Well \\
\hline DV98-90 & 82A-7 Well \\
\hline DV98-92 & Vl05 Separator \\
\hline DV98-95 & 73B-7 Well \\
\hline DV98-133 & 27-33 Well \\
\hline DV98-135 & 27-33 Well \\
\hline DV98- 138 & V101 Separator \\
\hline DV98-140 & 37-33 Well \\
\hline DV98-141 & 28-33 Well \\
\hline DV98-145 & 76A-7 Well \\
\hline DV98-147 & 63-7 Well \\
\hline DV98-148 & V102 + V103 Separator \\
\hline DV98-150 & 74-7 Well \\
\hline DV98-152 & 73-7 Well \\
\hline DV98-154 & 73B-7 Well \\
\hline DV98-156 & 82A-7 Well \\
\hline DV98-159 & V105 Separator \\
\hline DV99-182 & 76A-7 Well \\
\hline
\end{tabular}

\begin{tabular}{c} 
Date \\
$10 / 25 / 96$ \\
$10 / 25 / 96$ \\
$10 / 25 / 96$ \\
$10 / 25 / 96$ \\
$10 / 29 / 97$ \\
$10 / 29 / 97$ \\
$10 / 29 / 97$ \\
$10 / 29 / 97$ \\
$10 / 29 / 97$ \\
$10 / 29 / 97$ \\
$10 / 30 / 97$ \\
$10 / 30 / 97$ \\
$10 / 30 / 97$ \\
$10 / 30 / 97$ \\
$10 / 30 / 97$ \\
$04 / 28 / 98$ \\
$04 / 28 / 98$ \\
$04 / 28 / 98$ \\
$04 / 28 / 98$ \\
$04 / 28 / 98$ \\
$04 / 28 / 98$ \\
$04 / 28 / 98$ \\
$04 / 28 / 98$ \\
$04 / 29 / 98$ \\
$04 / 29 / 98$ \\
$04 / 29 / 98$ \\
$04 / 29 / 98$ \\
$10 / 20 / 98$ \\
$10 / 20 / 98$ \\
$10 / 21 / 98$ \\
$10 / 21 / 98$ \\
$10 / 21 / 98$ \\
$10 / 22 / 98$ \\
$10 / 22 / 98$ \\
$10 / 22 / 98$ \\
$10 / 22 / 98$ \\
$10 / 22 / 98$ \\
$10 / 22 / 98$ \\
$10 / 23 / 98$ \\
$10 / 23 / 98$ \\
$05 / 04 / 99$ \\
\hline
\end{tabular}

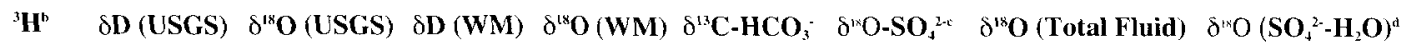

$\begin{array}{lllllll}\text { (TU) } & \text { (per mil) } & \text { (per mil) } & \text { (per mil) } & \text { (per mil) } & \text { (per mil) } & \text { (per mil) }\end{array}$

$\left({ }^{\circ} \mathrm{C}\right)$

$0.07 \quad--\quad-$

$\begin{array}{ll}-- & -124 \\ -- & -119\end{array}$

$\begin{array}{cc}-14.2 & -8.0 \\ -14.0 & --- \\ -14.3 & -8.0 \\ -14.3 & --- \\ -13.9 & -7.36 \\ -14.0 & -8.23 \\ -14.0 & -7.74 \\ -14.0 & -7.66\end{array}$

$--$

$--$

$---$

-7.94
-8.34

-8.34
-8.04

$-8.56$

$\begin{array}{lll}-13.9 & -8.66 & -8.29 \\ -14.0 & -7.39 & -8.46\end{array}$

$\begin{array}{lll}-13.9 & -8.06 & -7.96 \\ -14.7 & -7.85 & -9.05\end{array}$

$\begin{array}{lll}-14.7 & -7.85 & -9.05 \\ -14.5 & -7.23 & -8.86 \\ -14.5 & -7.35 & -9.01\end{array}$

$\begin{array}{lll}-14.5 & -7.23 & -8.86 \\ -14.5 & -7.35 & -9.01 \\ -14.5 & -6.91 & -9.21\end{array}$

$\begin{array}{lll}-14.5 & -6.91 & -9.21\end{array}$

$\begin{array}{ll}--- & -7.02 \\ - & -7.28\end{array}$

-7.02
-7.28

$\begin{array}{ll}-- & -7.05\end{array}$

$\begin{array}{ll}--- & -7.05 \\ -- & -7.60\end{array}$

$\begin{array}{ll}-- & -6.85\end{array}$

$\begin{array}{ll}- & -6.72\end{array}$

$---6.60$

$\begin{array}{ll}--- & -7.64\end{array}$

$---\quad-7.67$

---8.58
$-\quad-6.58$

$\begin{array}{ll}--- & -6.58 \\ - & -7.95\end{array}$

$\begin{array}{ll}--- & -7.28 \\ --- & -6.26\end{array}$

$$
--
$$$$
-13.51
$$$$
-13.97
$$$$
\text { --- }
$$$$
-13.29
$$$$
\begin{array}{r}
-13.52 \\
-14.40
\end{array}
$$$$
\begin{gathered}
-14.40 \\
--14.24
\end{gathered}
$$$$
-14.17
$$$$
\begin{aligned}
& -14.24 \\
& -14.33
\end{aligned}
$$$$
-14.19
$$$$
\begin{gathered}
--- \\
-13.82 \\
-13.63
\end{gathered}
$$$$
-13.63
$$$$
\begin{array}{r}
-13.63 \\
-13.74
\end{array}
$$$$
-13.55
$$$$
-13.19
$$$$
\begin{array}{r}
-13.44 \\
-13.55
\end{array}
$$$$
-8.16
$$$$
\begin{aligned}
& -13.55 \\
& -13.60
\end{aligned}
$$$$
\text { --- }
$$$$
-
$$$$
-9.48
$$$$
-8.69
$$$$
-9.05
$$$$
-8.55
$$$$
-8.51
$$$$
-8.44
$$$$
-8.60
$$$$
-7.88
$$$$
\begin{array}{lll}
10 / 23 / 98 & -- & -125
\end{array}
$$$$
-7.37
$$$$
\text { -.- }
$$ 


\section{Table 7: Continued}

${ }^{3} \mathrm{H}^{\mathrm{b}} \quad \delta \mathrm{D}$ (USGS) $\delta^{18} \mathrm{O}$ (USGS) $\delta \mathrm{D}(\mathrm{WM}) \delta^{18} \mathrm{O}(\mathrm{WM}) \delta^{13 \mathrm{C}-\mathrm{HCO}_{3}} \quad \delta^{110} \mathrm{O}-\mathrm{SO}_{4}^{2-4} \quad \delta^{18} \mathrm{O}$ (Total Fluid) $\delta^{11} \mathrm{O}\left(\mathrm{SO}_{4}{ }^{2}-\mathrm{H}_{2} \mathrm{O}\right)^{\mathrm{d}}$

$\begin{array}{ll}\text { Sample } & \text { Name or Description } \\ \text { DV99-184 } & 74-7 \text { Well } \\ \text { DV99-186 } & \text { V102 + V103 Separator } \\ \text { DV99-188 } & 63-7 \text { Well } \\ \text { DV99-190 } & \text { 73-7 Well } \\ \text { DV99-194 } & \text { V105 Separator } \\ \text { DV99-196 } & \text { 82A-7 Well } \\ \text { DV99-197 } & \text { 73B-7 Well } \\ \text { DV99-199 } & \text { 37-33 Well } \\ \text { DV99-200 } & \text { 28-33 Well } \\ \text { DV99-204 } & \text { V101 Separator } \\ \text { DV74782786-brine 2 } & \text { 74-7 Well Archived } \\ \text { DV76781986-brine 4 } & \text { 76-7 Well Archived } \\ \text { DV453382186-brine 6 } & 45-33 \text { Well Archived } \\ \text { DV73782886-brine 8 } & 73-7 \text { Well Archived } \\ \text { DV321882686-brine 10 32-18 Well Archived } \\ \text { DV651882686-brine 12 } 65-18 \text { Well Archived } \\ \text { No number } & 28-33 \text { Well Archived }\end{array}$

Mean brine temp., ${ }^{\circ} \mathrm{C} \quad$ Sulfate-oxygen geothermomete

$\begin{array}{ccc}\text { Date } & \text { (TU) } & \text { (per mil) } \\ 05 / 04 / 99 & --- & -126 \\ 05 / 04 / 99 & -- & -125 \\ 05 / 04 / 99 & -- & -125 \\ 05 / 04 / 99 & -- & -124 \\ 05 / 05 / 99 & -- & -124 \\ 05 / 05 / 99 & -- & -125 \\ 05 / 05 / 99 & -- & -124 \\ 05 / 05 / 99 & -- & -126 \\ 05 / 05 / 99 & -- & -127 \\ 05 / 05 / 99 & -- & -128 \\ 08 / 27 / 86 & -- & -130 \\ 08 / 19 / 86 & \ldots- & -130 \\ 08 / 21 / 86 & -- & -131 \\ 08 / 28 / 86 & --- & -129 \\ 08 / 26 / 86 & -- & -130 \\ 08 / 26 / 86 & --- & -131 \\ 09 / 23 / 93 & -- & -133 \\ \mathrm{n}=3 & -- & ---\end{array}$

per mil)

$-13.08$

$-12.96$

-12.96
-12.89

$-13.04$

$-13.07$

$-12.97$

$-13.80$

$-13.71$

$-13.71$

$-14.11$

$-14.09$

$-14.44$

-14.20
-14.07

$-14.11$

$-16.46$

$10 / 25 / 96$

$10 / 25 / 96$

$10 / 25 / 96$

$10 / 25 / 96$

$10 / 29 / 97$

$10 / 29 / 97$

$10 / 29 / 97$

$10 / 29 / 97$

$10 / 29 / 97$

$10 / 29 / 97$

10/30/97

$10 / 30 / 97$

$10 / 30 / 97$
$10 / 30 / 97$

$04 / 28 / 98$

$04 / 28 / 98$

$04 / 28 / 98$

$04 / 28 / 98$

$04 / 28 / 98$

$04 / 28 / 98$

$04 / 28 / 98$

$04 / 29 / 98$

---

$$
\text { --- }
$$

(per mil)

$-8.28$

$-7.82$

$---$

$-8.08$

$-8.25$

$-8.00$

$-8.80$

$-8.31$

$-8.50$

$-8.27$$$
\text { --- }
$$

---

---

74-7 Well

73-7 Well 
Table 7: Continued

$\begin{array}{ll}\text { Sample } & \text { Name or Description } \\ \text { DV98-91 } & 82 \mathrm{~A}-7 \text { Well } \\ \text { DV98-93 } & \text { V105 Separator } \\ \text { DV98-94 } & \text { 73B-7 Well } \\ \text { DV98-101 } & 28-33 \text { Well } \\ \text { DV98-134 } & 27-33 \text { Well } \\ \text { DV98-136 } & \text { 27-33 Well } \\ \text { DV98-137 } & \text { V101 Separator } \\ \text { DV98-139 } & 37-33 \text { Well } \\ \text { DV98-142 } & 28-33 \text { Well } \\ \text { DV98-144 } & 76 \text { A-7 Well } \\ \text { DV98-146 } & \text { V102 + V103 Separator } \\ \text { DV98-149 } & 63-7 \text { Well } \\ \text { DV98-151 } & 74-7 \text { Well } \\ \text { DV98-153 } & 73-7 \text { Well } \\ \text { DV98-155 } & 73 \text { B-7 Well } \\ \text { DV98-157 } & 82 A-7 \text { Well } \\ \text { DV98-158 } & \text { V105 Separator } \\ \text { DV99-183 } & 76 \text { A-7 Well } \\ \text { DV99-185 } & 74-7 \text { Well } \\ \text { DV99-187 } & \text { V102 + V103 Separator } \\ \text { DV99-189 } & 63-7 \text { Well } \\ \text { DV99-191 } & 73-7 \text { Well } \\ \text { DV99-192 } & 73 \text { B-7 Well } \\ \text { DV99-193 } & \text { V105 Separator } \\ \text { DV99-195 } & 82 \mathrm{~A}-7 \text { Well } \\ \text { DV99-201 } & 28-33 \text { Well } \\ \text { DV99-202 } & 37-33 \text { Well } \\ \text { DV99-203 } & \text { V101 Separator } \\ \text { DV74782786-cond 1 } & 74-7 \text { Well Archived } \\ \text { DV76781986-cond 3 } & 76-7 \text { Well Archived } \\ \text { DV453382886-cond 5 } & 45-33 \text { Well Archived } \\ \text { DV73782886-cond 7 } & 73-7 \text { Well Archived } \\ \text { DV321882686-cond 9 } & 32-18 \text { Well Archived } \\ \text { DV651882686-cond I1 } & 65-18 \text { Well Archived } \\ & \end{array}$

Date
$04 / 29 / 98$
$04 / 29 / 98$
$04 / 29 / 98$
$04 / 30 / 98$
$10 / 20 / 98$
$10 / 20 / 98$
$10 / 21 / 98$
$10 / 21 / 98$
$10 / 21 / 98$
$10 / 22 / 98$
$10 / 22 / 98$
$10 / 22 / 98$
$10 / 22 / 98$
$10 / 22 / 98$
$10 / 22 / 98$
$10 / 23 / 98$
$10 / 23 / 98$
$05 / 04 / 99$
$05 / 04 / 99$
$05 / 04 / 99$
$05 / 04 / 99$
$05 / 04 / 99$
$05 / 04 / 99$
$05 / 05 / 99$
$05 / 05 / 99$
$05 / 05 / 99$
$05 / 05 / 99$
$05 / 05 / 99$
$08 / 27 / 86$
$08 / 19 / 86$
$08 / 28 / 86$
$08 / 28 / 86$
$08 / 26 / 86$
$08 / 26 / 86$

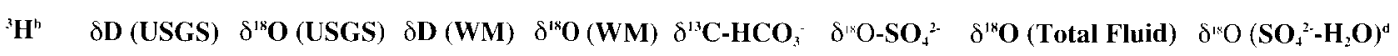

$\begin{array}{lllllll}\text { (TU) } & \text { (per mil) } & \text { (per mil) } & \text { (per mil) } & \text { (per mil) } & \text { (per mil) } & \text { (per mil) }\end{array}$

$\left({ }^{\circ} \mathrm{C}\right)$

DV651882686-cond 11 65-18 Well Archived

Injection Well/Power Plant Fluids

$\begin{array}{ll}\text { DV96-2 } & \text { Condensate from plant } \\ \text { DV96-3 } & \text { LP Brine @ Plant } \\ \text { DV96-4 } & \text { 45-5 Injection Well } \\ \text { DV96-5 } & \text { Lamb I Injection Well } \\ \text { DV96-6 } & \text { 65-18 Injection Well } \\ \text { DV97-32 } & \text { Condensate from plant } \\ \text { DV97-33 } & \text { LP Brine @ Plant }\end{array}$

$08 / 26 / 86$

$\begin{array}{cccccc}\text { (per mil) } & \text { (per mil) } & (\text { per mil) } & (\text { per mil) } & (\text { per mil) } & \text { (per mil) } \\ -14.57 & --- & --- & --- & --- & -- \\ -15.97 & -- & -- & -- & -- & --\end{array}$

$---133 \quad-15.97$

$\begin{array}{lll}-- & -131 & -15.99 \\ - & -133 & -16.50\end{array}$

$\begin{array}{lll}--133 & -16.50\end{array}$

$-16.90$

$-17.10$

$-16.58$

-16.69
-16.60

-16.60
-16.20

-16.20
-15.97

$-15.89$

$-16.06$

$-15.26$

-15.74
-15.83

$-15.91$

$-16.10$

$-15.69$

$-16.04$

-15.93
-15.12

$-14.47$

$-15.86$

$-15.66$

$-16.46$

$-16.59$

$-16.57$

$-17.39$

$-17.56$

$-17.5$

$-17.3$

$---$


Table 7: Continued

$\begin{array}{ll}\text { Sample } & \text { Name or Description } \\ \text { DV97-34 } & 25-5+45-5 \text { Injectate } \\ \text { DV97-35 } & 25-5+45-5 \text { Injectate } \\ \text { DV97-36 } & 65-18 \text { Injection Well } \\ \text { DV97-37 } & \text { 32-18 Injection Well } \\ \text { DV97-40 } & \text { LP Brine @ Plant } \\ \text { DV97-41 } & \text { Condensate from plant } \\ \text { DV97-42 } & \text { High P Brine @ plant } \\ \text { DV98-97 } & \text { Condensate from plant } \\ \text { DV98-98 } & \text { LP Brine @ Plant } \\ \text { DV98-143 } & \text { 25-5 Injection Well } \\ \text { DV98-161 } & \text { Condensate from plant } \\ \text { DV98-162 } & \text { LP Brine @ Plant } \\ \text { DV98-163 } & \text { 65-18 Injection Well } \\ \text { DV99-198 } & 65-18 \text { Injection Well } \\ \text { DV99-205 } & \text { 25-5 + 45-5 Injectate } \\ \text { DV99-206 } & \text { LP Brine @ Plant } \\ \text { DV99-207 } & \text { Condensate from plant } \\ \text { DV99-208 } & 52-18+41-18 \text { Injectate }\end{array}$

\begin{tabular}{ccc} 
Date & $($ TU) & (per mil) \\
$10 / 31 / 97$ & 0.06 & -120 \\
$10 / 31 / 97$ & --- & -121 \\
$10 / 31 / 97$ & --- & -126 \\
$10 / 31 / 97$ & --- & -126 \\
$10 / 31 / 97$ & $-\mathbf{0 . 0 3}$ & -127 \\
$10 / 31 / 97$ & -- & -89 \\
$10 / 31 / 97$ & --- & -129 \\
$04 / 29 / 98$ & -- & -91 \\
$04 / 29 / 98$ & --- & -124 \\
$10 / 21 / 98$ & -- & -124 \\
$10 / 23 / 98$ & 0.79 & -95 \\
$10 / 23 / 98$ & --- & -123 \\
$10 / 23 / 98$ & 0.62 & -110 \\
$05 / 05 / 99$ & --- & -112 \\
$05 / 06 / 99$ & -0.10 & -123 \\
$05 / 06 / 99$ & --- & -123 \\
$05 / 06 / 99$ & 1.08 & -95 \\
$05 / 06 / 99$ & -- & -123 \\
& & \\
$10 / 24 / 96$ & 0.11 & --- \\
$10 / 31 / 97$ & --- & -125 \\
$10 / 31 / 97$ & 0.18 & -127 \\
$11 / 05 / 97$ & -- & -161 \\
$11 / 05 / 97$ & -- & -134 \\
$11 / 05 / 97$ & 0.41 & -126 \\
$11 / 05 / 97$ & -- & -114 \\
$11 / 07 / 97$ & -- & -124 \\
$04 / 29 / 98$ & --- & -127 \\
$04 / 29 / 98$ & --- & -133 \\
$04 / 29 / 98$ & --- & -148 \\
$04 / 30 / 98$ & --- & -141 \\
$04 / 30 / 98$ & --- & -128 \\
$04 / 30 / 98$ & --- & -126 \\
$05 / 01 / 98$ & 0.83 & -135 \\
$05 / 05 / 98$ & -- & -132 \\
$05 / 06 / 98$ & -- & -120 \\
$10 / 23 / 98$ & 0.42 & -128 \\
$10 / 26 / 98$ & --- & -134 \\
$10 / 28 / 98$ & --- & -135 \\
$05 / 04 / 99$ & --- & -127 \\
& & \\
\hline
\end{tabular}

\begin{tabular}{ll}
\multicolumn{2}{l}{ Other Geothermal and On-Site Water Wells } \\
\hline DV96-1 & Domestic Well \\
DV97-38 & Domestic Well \\
DV97-39 & Goerenger Well \\
DV97-53 & $46-32$ Well \\
DV97-54 & $27-32$ Well \\
DV97-55 & $27-32$ Well \\
DV97-59 & $45-$ W-5 Well \\
DV97-67 & $66-21$ Well \\
DV98-96 & Goerenger Well \\
DV98-99 & $27-32$ Well \\
DV98-100 & $46-32$ Well \\
DV98-102 & $45-14$ Well \\
DV98-103 & $45-14$ Well \\
DV98-104 & $66-21$ Well \\
DV98-11I & $62-21$ Well \\
DV98-122 & $97-2$ Well \\
DV98-123 & $32-6$ Well \\
DV98-160 & Goerenger Well \\
DV98-168 & $38-32$ Well \\
DV98-175 & $62-21$ Well \\
DV99-181 & Goerenger Well
\end{tabular}

$05 / 04 / 99$

${ }^{3} \mathrm{H}^{h} \quad \delta \mathrm{D}$ (USGS) $\delta^{18} \mathrm{O}$ (USGS) $\delta \mathrm{D}(\mathrm{WM}) \quad \delta^{18} \mathrm{O}$ (WM) $\delta^{13} \mathrm{C}-\mathrm{HCO}_{3} \quad \delta^{18 \mathrm{O}-\mathrm{SO}_{4}{ }^{2 \cdot}} \quad \delta^{18} \mathrm{O}$ (Total Fluid) $\quad \delta^{110} \mathrm{O}\left(\mathrm{SO}_{4}^{2} \cdot \mathrm{H}_{2} \mathrm{O}\right)^{d}$ 


\section{Table 7: Continued}

\section{Sample}

Background Springs

DV97-46

DV97-47

DV97-48

DV97-50

DV97-51a

DV97-51b

DV97-52

DV97-56

DV97-60

DV97-61

DV97-62

DV97-63

DV97-64

DV97-65

DV97-66

DV97-68

DV97-69

DV $97-72$

DV98-106

DV98-112

DV98-113

DV98-114

DV98-117

DV98-118

DV98-120

DV98-128

DV98-129

DV98-131

DV98-132

DV98-169

DV $98-170$

DV98-176

DV98-177

DV98-178

DV98-179

DV99-209

DV99-210

DV99-211
Name or Description

Sou Hot Spring

Sou Hot Spring

Hyder Hot Spring

Edward Creek Spring

Old Man Spring

Horse Heaven Spring

Dead Travertine Spring, Upper

Fault Line Spring

Lower Ranch Hot Spring

McCoy Hot Spring

Kyle Spring

Dago Spring

Mustang Spring

Kitten Spring

Big Horn Spring

Dixie Hot Spring

Horse Creek Spring

Stu's Seep

Hyder Hot Spring

Lower Ranch Hot Spring

McCoy Hot Spring

Sou Hot Spring

Big Horn Spring

Dixie Hot Spring

Jersey Hot Spring

Upper Jersey Seep

Spring in Spring Canyon

Wild Rose Spring

Lofthouse Spring

Not-So-OK Spring

War Canyon Spring

Pine Spring

Basalt Spring

Upper Cherry Spring

Dead Travertine Spring, Upper

Dead Travertine Spring, Road

Upper Spring, Lower Ranch

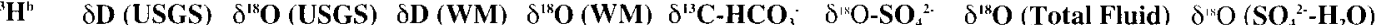
(per mil) (per mil)

(per mil) (per mil) (per mil) (per mil)

(per mil)

$\left({ }^{\circ} \mathrm{C}\right)$

\begin{tabular}{|c|c|c|}
\hline-- & --- & -1.23 \\
\hline --- & --- & -3.35 \\
\hline$\cdots$ & --- & -3.87 \\
\hline--- & $\cdots$ & --- \\
\hline-- & --- & -- \\
\hline --- & --- & -10.46 \\
\hline--- & --- & -10.59 \\
\hline-- & --- & -3.07 \\
\hline$\cdots$ & --- & -3.64 \\
\hline --- & --- & -3.31 \\
\hline --- & --- & -3.96 \\
\hline --- & --- & -6.46 \\
\hline$\cdots$ & --- & --- \\
\hline -- & --- & -9.54 \\
\hline-- & --- & -11.11 \\
\hline-- & $\cdots$ & -7.64 \\
\hline-- & --- & -6.93 \\
\hline--- & --- & -16.09 \\
\hline$\cdots$ & --- & -- \\
\hline$\cdots$ & --- & -3.12 \\
\hline--- & --- & -2.93 \\
\hline--- & --- & -3.68 \\
\hline --- & --.. & -2.60 \\
\hline --. & $\ldots$ & -7.52 \\
\hline -.. & $\ldots$ & -6.67 \\
\hline$\ldots$ & --- & -3.51 \\
\hline-- & --- & --- \\
\hline$\cdots$ & $\cdots$ & -9.52 \\
\hline--- & --- & -11.98 \\
\hline--- & --- & -11.41 \\
\hline --- & --- & -11.95 \\
\hline --- & --- & -12.99 \\
\hline --- & --- & -13.46 \\
\hline --- & $\cdots$ & -13.01 \\
\hline--- & $-\cdots$ & -12.72 \\
\hline --- & --- & -2.37 \\
\hline$\cdots$ & $\cdots$ & -3.34 \\
\hline & & -3.69 \\
\hline
\end{tabular}

$\begin{array}{lcc}11 / 03 / 97 & --- & -129 \\ 11 / 03 / 97 & -\mathbf{0 . 0 2} & -131 \\ 11 / 03 / 97 & 0.12 & -135 \\ 11 / 04 / 97 & 0.05 & -122 \\ 11 / 04 / 97 & --- & -112 \\ 11 / 04 / 97 & 0.09 & -132 \\ 11 / 04 / 97 & --- & -123 \\ 11 / 05 / 97 & -- & -123 \\ 11 / 06 / 97 & 0.02 & -132 \\ 11 / 06 / 97 & 0.07 & -131 \\ 11 / 06 / 97 & --- & -130 \\ 11 / 06 / 97 & 0.04 & -122 \\ 11 / 06 / 97 & --- & -120 \\ 11 / 06 / 97 & 9.2 & -116 \\ 11 / 06 / 97 & 0.11 & -119 \\ 11 / 07 / 97 & --- & -117 \\ 11 / 07 / 97 & 0.16 & -128 \\ 11 / 07 / 97 & 10.2 & -119 \\ 04 / 30 / 98 & --- & -111 \\ 04 / 30 / 98 & --- & -134 \\ 05 / 04 / 98 & --- & -130 \\ 05 / 04 / 98 & --- & -128 \\ 05 / 04 / 98 & --- & -129 \\ 05 / 04 / 98 & -- & -117 \\ 05 / 05 / 98 & --- & -127 \\ 05 / 05 / 98 & 1.1 & -128 \\ 05 / 06 / 98 & --- & -126 \\ 05 / 06 / 98 & --- & -123 \\ 05 / 07 / 98 & 4.46 & -118 \\ 05 / 07 / 98 & --- & -114 \\ 10 / 27 / 98 & 9.2 & -113 \\ 10 / 27 / 98 & 3.45 & -126 \\ 10 / 28 / 98 & 6.73 & -119 \\ 10 / 28 / 98 & --- & -122 \\ 10 / 28 / 98 & --- & -119 \\ 05 / 07 / 99 & -- & -122 \\ 05 / 08 / 99 & --- & -117 \\ 05 / 09 / 99 & --- & -130\end{array}$

-15.7
-16.0
-15.6
-15.4
-14.3
-16.8
-15.4
-14.9
-16.4
-16.4
-16.1
-15.7
-14.9
-14.3
-15.2
-14.7
-16.1
-15.5
-13.4
-15.5
-16.4
-16.2
-16.0
-14.7
-16.0
-15.9
-15.5
-15.8
-14.6
-14.8
-14.8
-16.3
-15.4
-16.0
-15.6
-15.0
-13.8
-16.6

$$
\begin{gathered}
-9.62 \\
+10.48 \\
+4.88 \\
--- \\
-- \\
--- \\
--- \\
-- \\
+3.46 \\
+1.63 \\
+3.55 \\
--- \\
--- \\
--- \\
--- \\
--- \\
-2.87 \\
--- \\
-- \\
-- \\
-0.52 \\
-- \\
+10.08 \\
+7.52 \\
--- \\
-6.15 \\
--- \\
--- \\
+0.29 \\
-- \\
--- \\
--- \\
--- \\
--- \\
--- \\
---
\end{gathered}
$$

\begin{tabular}{|c|c|}
\hline -.- & 257 \\
\hline --- & 33 \\
\hline --- & 68 \\
\hline --- & $\cdots$ \\
\hline --- & $\cdots$ \\
\hline --- & -- \\
\hline --- & -- \\
\hline --- & --- \\
\hline--- & 73 \\
\hline -.. & 87 \\
\hline --. & 74 \\
\hline --- & --- \\
\hline --- & $\cdots$ \\
\hline --- & -- \\
\hline --- & --- \\
\hline --- & -- \\
\hline --- & 133 \\
\hline --- & --- \\
\hline$\cdots$ & --- \\
\hline --- & -- \\
\hline --. & 105 \\
\hline --- & $\ldots$ \\
\hline --- & 35 \\
\hline --- & 57 \\
\hline --- & --- \\
\hline --. & 181 \\
\hline --- & --- \\
\hline --- & $\cdots$ \\
\hline --- & --- \\
\hline --- & --- \\
\hline --- & --- \\
\hline --- & --- \\
\hline --- & --- \\
\hline --- & $\cdots$ \\
\hline --- & -- \\
\hline --- & -- \\
\hline --- & --- \\
\hline & \\
\hline
\end{tabular}

$05 / 09 / 99$

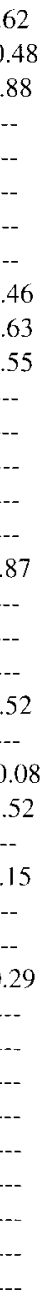


Table 7: Continued

\section{Sample}

Background Wells

DV97-49

DV97-57

DV97-70

DV97-71

DV98-115

DV98- 116

\section{DV98-172 \\ Background Streams/Rain}

DV97-58
DV98-107

DV98-110

DV98-119

DV98-121

DV98-125

DV98-126

DV98-127

DV98-130

U. DV98-17

DV98-17

DV98-174

DV98-180

DV99-213

\section{Fumaroles}

DV97-43

DV97-44

DV98-105

DV98- 108

DV98-109

DV98-124

DV98-165

DV98-166

\section{Backgrou}

\section{Name or Description}

Hole in the Wall \#2 Well

Bolivia Artesian Well

Shaw Well

Irrigation Well

Brinkerhoff Well

Bernice Well
Flowing well @ AA Tank

$\delta^{18} \mathrm{O}$ (USGS)

(per mil)

(per mil) (per mil) (per mil)

$\delta^{\wedge} \mathrm{O}-\mathrm{SO}_{4}^{2-}$

$\delta^{18} \mathrm{O}$ (Total Fluid) $\delta^{110} \mathrm{O}\left(\mathrm{SO}_{4}{ }^{2-}-\mathrm{H}_{2} \mathrm{O}\right)^{\mathrm{d}}$

$\begin{array}{ll}11 / 04 / 97 & 0.46 \\ 11 / 05 / 97 & 0.10\end{array}$

$11 / 07 / 97$

$11 / 07 / 97$

$05 / 04 / 98$

$05 / 04 / 98$

$10 / 27 / 98$

0.10

$-0.04$

0.75

-121
-121
-129
-134
-132
-128
-112

-15.3
-14.8
-16.6
-16.6
-15.9
-15.9
-13.9

---
---
---
--
--
--
--

---
---
---
---
---
---
--

-6.09
-8.96
-8.93
-9.47
-2.41
-4.41
-9.22

---
+2.84
---
---
+0.75
---
---

(per mil)

$\left({ }^{\circ} \mathrm{C}\right)$

\begin{tabular}{|c|c|c|c|c|c|c|c|c|c|}
\hline $11 / 05 / 97$ & --- & -118 & -14.6 & -.- & --- & -9.95 & --- & --- & \\
\hline $04 / 30 / 98$ & --. & -109 & -12.8 & --- & --- & --- & --- & --- & \\
\hline $05 / 01 / 98$ & $\ldots$ & -115 & -14.6 & --. & -.- & --- & --- & --- & $\ldots$ \\
\hline $05 / 05 / 98$ & --- & -110 & -13.7 & -.- & $\ldots$ & -4.43 & +3.94 & --- & $\ldots$ \\
\hline $05 / 05 / 98$ & 11.3 & -109 & -14.2 & --- & --- & -7.19 & --. & --- & -- \\
\hline $05 / 06 / 98$ & 11.2 & -64 & -8.0 & --- &.-- & --- & --. & --- & $\ldots$ \\
\hline $05 / 06 / 98$ & -.- & -115 & -15.2 & --- & --- & -6.86 & --- & --- & \\
\hline 05/06/98 & 10.4 & -111 & -14.6 & --- & --- & -6.50 & --- & --- & \\
\hline $05 / 06 / 98$ & --- & -113 & -14.9 & --- & --- & --- & --- & --- & -- \\
\hline $10 / 27 / 98$ & --- & -115 & -14.8 & $\cdots$ & $\cdots$ & --- & --- & --- & -- \\
\hline $10 / 27 / 98$ & --- & -112 & -13.8 & $\cdots$ & --- & -8.41 & --- & $\cdots$ & $\cdots$ \\
\hline $10 / 27 / 98$ & --- & -110 & -13.2 & --- & --- & -5.99 & --- & & --- \\
\hline $10 / 28 / 98$ & --- & -120 & -15.8 & --- & --- & -9.30 & --. & $\ldots$ & --- \\
\hline $05 / 10 / 99$ & --- & -92 & -7.8 & --- & --- & 1.67 & $\cdots$ & --- & --- \\
\hline
\end{tabular}

Tritium analyzed by University of Miami (error $= \pm 10 \%$ ), stable isotopes of water analyzed by T. Coplan. USGS, Reston. Virginia (error $= \pm 1$ per mil deuterium and \pm 0.15 per mil oxygen-18) and Western Michigan University (error $= \pm 1$ per mil deuterium and \pm 0.25 per mil oxygen-18): carbon-13 analyses performed by Geochron

Laboratories, Cambridge, Massachusetts (error $= \pm 0.2$ per mil); oxygen -18 in sulfate analyses by USGS (error $= \pm 0.15$ per mil).

"Negative values shown in bold should be considered to be 0.00 TU for any calculations.

"Values in bold are averages of 1997 and 1998 analyses for the same well to perform the sulfate-oxygen isotope geothermometer calculations.

"Sulfate oxygen isotope geothermometer of McKenzie and Truesdell (1977). Estimated reservoir temperatures of 1997 and 1998 brine samples are too high because the oxygen-18 value of unflashed reservoir water has increased due to production (Kennedy et al, 1999). Temperatures estimated from 1986 archived samples (bold) are most representative of the pre-production resevoir temperature and isotope

composition. Temperatures in italics are probably not reliable due to requilibration, evaporation. or mixing of different waters. 
Table 8: Gas Geochemistry and Geothermometer Calculations for Various Geothermal and Regional Fluids, Dixie Valley Region, Nevada (values in mol\% dry gas unless otherwise noted).

\begin{tabular}{|c|c|c|c|c|c|c|c|c|c|c|c|c|c|c|c|c|}
\hline Sample & Name or Description & Date & Laboratory & $\begin{array}{l}\text { Sampling } \\
\text { Temp. }\left({ }^{\circ} \mathrm{C}\right)\end{array}$ & $\begin{array}{c}\text { Steam } \\
\text { Fraction (y) }\end{array}$ & $\begin{array}{c}\text { Steam/Gas } \\
\text { (molar) }\end{array}$ & $\begin{array}{c}\mathrm{H}_{2} \mathrm{O} \\
\text { (mol\%, wet) }\end{array}$ & $\mathrm{CO}_{2}$ & $\mathrm{H}_{2} \mathrm{~S}$ & $\mathbf{H}_{2}$ & $\mathrm{CH}_{\triangleleft}$ & $\mathrm{C}_{2} \mathbf{H}_{4}$ & $\mathbf{N}_{2}$ & $\mathbf{N H}_{3}$ & $\mathbf{O}_{2}$ & Ar \\
\hline \multicolumn{17}{|c|}{ Production and On-Site Wells } \\
\hline DIXE102G & V102+ V103 Separator & $10 / 02 / 95$ & LANL & -- & 0.153 & 1021 & 99.9 & 96.6 & 2.260 & 0.0000 & 0.003 & 0 & 0.159 & 0.909 & 0.0364 & 0.0022 \\
\hline DV96-7a & 76-7 Well & $10 / 25 / 96$ & LANL & 163 & 0.184 & 604 & 99.8 & 97.5 & 0.560 & 0.0125 & 0.459 & 0.01790 & 0.802 & 0.687 & 0.0300 & 0.0134 \\
\hline DV96-7b & 76-7 Well & $10 / 25 / 96$ & LANL & 163 & --- & 703 & 99.9 & 96.9 & 0.633 & 0.0140 & 0.563 & 0.02086 & 1.011 & 0.830 & 0.0491 & 0.0173 \\
\hline DV96-7c & 76-7 Well & $10 / 25 / 96$ & LANL & 163 & -.- & 496 & 99.8 & 96.6 & 0.563 & 0.0195 & 0.823 & 0.03027 & 1.272 & 0.626 & 0.0228 & 0.0325 \\
\hline DV96-10a & V 101 Separator & $10 / 25 / 96$ & LANL & 160 & 0.159 & 512 & 99.8 & 96.6 & 1.147 & 0.0290 & 0.414 & 0.00901 & 1.318 & 0.619 & 0.0340 & 0.0252 \\
\hline DV96-10b & V101 Separator & $10 / 25 / 96$ & LANL & 160 & --- & 1438 & 99.9 & 96.4 & 0.775 & 0.0164 & 0.225 & 0.00473 & 0.757 & 1.792 & 0.0201 & 0.0302 \\
\hline \multicolumn{17}{|c|}{ Average 1996 Geothermometer $(n=5)$} \\
\hline DV97-12(2) & 73-7 Well & $10 / 29 / 97$ & USGS & --- & 0.158 & 816 & 99.9 & 93.9 & 1.976 & 0.0390 & 0.941 & --- & 2.049 & 1.024 & 0.0038 & 0.0392 \\
\hline DV97.15 & 74-7 Well & $10 / 29 / 97$ & LANL & --- & 0.163 & 542 & 99.8 & 96.8 & 0.739 & 0.0152 & 0.572 & 0.01879 & 0.966 & 0.408 & 0.0168 & 0.0542 \\
\hline DV97-15 (2) & 74-7 Well & $10 / 29 / 97$ & USGS & --- & --- & 551 & 99.8 & 94.8 & 1.095 & 0.0238 & 1.039 & & 2.058 & 0.879 & 0.0031 & 0.0401 \\
\hline DV97-17 & V102+V103 Separator & 10/29/97 & LANL & --- & 0.161 & 476 & 99.8 & 96.6 & 0.765 & 0.0122 & 0.634 & 0.02021 & 1.257 & 0.651 & 0.0082 & 0.0231 \\
\hline DV97-17 & V102+V103 Separator & $10 / 29 / 97$ & USGS & --- & --- & 487 & 99.8 & 95.6 & 0.683 & 0.0208 & 0.979 & --- & 1.931 & 0.748 & 0.0000 & 0.0340 \\
\hline DV97-19 & V105 Separator & 10/29/97 & USGS & --. & 0.151 & 545 & 99.8 & 95.7 & 0.816 & 0.0231 & 0.838 & --- & 1.918 & 0.693 & 0.0022 & 0.0382 \\
\hline DV97-21 (1) & $82 \mathrm{~A}-7$ Well & $10 / 29 / 97$ & USGS & --- & 0.159 & 540 & 99.8 & 93.9 & 1.093 & 0.0177 & 0.802 & --- & 3.64 & 0.535 & 0.0000 & 0.0579 \\
\hline DV97-22 & 73B-7 Well & $10 / 29 / 97$ & USGS & --- & 0.160 & 626 & 99.8 & 91.2 & 1.145 & 0.0322 & 0.739 & --- & 6.00 & 0.811 & 0.0000 & 0.0866 \\
\hline DV97-24 & V101 Separator & $10 / 30 / 97$ & USGS & --- & 0.164 & 476 & 99.8 & 93.6 & 1.026 & 0.0206 & 0.519 & --- & 4.11 & 0.627 & 0.0000 & 0.0650 \\
\hline DV97-27 & 27-33 Well & $10 / 30 / 97$ & USGS & --- & 0.157 & 376 & 99.7 & 95.2 & 0.929 & 0.0310 & 1.028 & $\ldots$ & 2.22 & 0.531 & 0.0230 & 0.0447 \\
\hline DV97-28 & 37-33 Well & $10 / 30 / 97$ & USGS & --- & 0.159 & 448 & 99.8 & 94.5 & 1.200 & 0.0252 & 0.590 & --- & 3.06 & 0.560 & 0.0014 & 0.0554 \\
\hline \multirow{2}{*}{\multicolumn{17}{|c|}{ Average 1997 Geothermometer $(n=11)$}} \\
\hline & & & & & & & & & & & & & & & & \\
\hline DV98-74 & V101 Separator & $04 / 28 / 98$ & LANL & 160 & 0.157 & 499 & 99.8 & 96.7 & 1.194 & 0.0160 & 0.458 & 0.00880 & 1.467 & 0.109 & 0.0064 & 0.0298 \\
\hline DV98-74 & V101 Separator & $04 / 28 / 98$ & USGS & --- & --- & 441 & 99.8 & 95.0 & 1.188 & 0.0259 & 0.676 & --- & 2.17 & 0.851 & 0.0000 & 0.0432 \\
\hline DV98-76 & 27-33 Well & $04 / 28 / 98$ & USGS & --- & 0.155 & 369 & 99.7 & 95.1 & 1.068 & 0.0253 & 1.007 & --- & 2.01 & 0.703 & 0.0000 & 0.0414 \\
\hline DV98-78 & 37-33 Well & $04 / 28 / 98$ & USGS & --- & 0.156 & 435 & 99.8 & 95.2 & 1.207 & 0.0332 & 0.610 & --- & 2.21 & 0.726 & 0.0000 & 0.0427 \\
\hline DV98-81 & 76A-7 Well & $04 / 28 / 98$ & LANL & & 0.157 & 433 & 99.8 & 96.1 & 0.609 & 0.0140 & 1.050 & 0.03780 & 1.555 & 0.112 & 0.0065 & 0.0275 \\
\hline DV98-81 & $76 \mathrm{~A}-7$ Well & $04 / 28 / 98$ & USGS & --- & --- & 450 & 99.8 & 95.5 & 0.635 & 0.0173 & 1.248 & --- & 1.690 & 0.839 & 0.0000 & 0.0331 \\
\hline DV98-83 & V102+V 103 Separator & $04 / 28 / 98$ & USGS & --- & 0.150 & 523 & 99.8 & 95.3 & 0.795 & 0.0212 & 1.041 & --- & 1.796 & 0.970 & 0.0039 & 0.0350 \\
\hline DV98-85 & $74-7$ Well & $04 / 28 / 98$ & USGS & --- & 0.158 & 464 & 99.8 & 95.5 & 0.753 & 0.0235 & 1.103 & --- & 1.782 & 0.742 & 0.0047 & 0.0353 \\
\hline DV98-87 & 63-7 Well & $04 / 28 / 98$ & USGS & --- & 0.154 & 518 & 99.8 & 95.5 & 0.685 & 0.0343 & 0.959 & --- & 1.746 & 1.001 & 0.0043 & 0.0336 \\
\hline DV98-93 & V105 Separator & $04 / 29 / 98$ & USGS & --- & 0.150 & 57.3 & 99.8 & 95.2 & 0.912 & 0.0210 & 0.864 & --- & 1.860 & 1.056 & 0.0129 & 0.0369 \\
\hline DV98-94 & 73B-7 Well & $04 / 29 / 98$ & USGS & --- & 0.152 & 567 & 99.8 & 95.2 & 1.081 & 0.0827 & 0.826 & --- & 1.781 & 0.980 & 0.0000 & 0.0356 \\
\hline DV98-137 & V101 Separator & $10 / 21 / 98$ & LANL & --- & 0.160 & 453 & 99.8 & 96.0 & 1.167 & 0.0180 & 0.543 & 0.01240 & 1.630 & 0.516 & 0.0056 & 0.0309 \\
\hline DV98-139 & 37-33 Well & $10 / 21 / 98$ & LANL & --- & 0.162 & 357 & 99.7 & 96.0 & 1.203 & 0.0309 & 0.581 & 0.01383 & 1.690 & 0.526 & 0.0059 & 0.0309 \\
\hline DV98-142 & $28-33 \mathrm{Well}$ & $10 / 21 / 98$ & LANL & --- & --- & 423 & 99.8 & 96.0 & 0.966 & 0.0640 & 0.406 & 0.00620 & 1.766 & 0.602 & 0.0240 & 0.0300 \\
\hline DV98-144 & 76A-7 Well & $10 / 22 / 98$ & LANL & --- & 0.158 & 462 & 99.8 & 95.8 & 0.643 & 0.0162 & 1.090 & 0.04098 & 1.603 & 0.669 & 0.0031 & 0.0295 \\
\hline DV98-146 & V102 + V103 Separator & $10 / 22 / 98$ & LANL & --- & 0.164 & 515 & 99.8 & 96.5 & 0.742 & 0.0118 & 0.584 & 0.01994 & 1.115 & 0.761 & 0.0078 & 0.0210 \\
\hline DV98-149 & 63-7 Well & $10 / 22 / 98$ & LANL & --- & --- & 462 & 99.8 & 94.5 & 0.517 & 0.0150 & 0.572 & 0.01830 & 1.230 & 0.826 & 0.0140 & 0.0210 \\
\hline DV98-153 & 73-7 Well & $10 / 22 / 98$ & LANL & --- & --- & 505 & 99.8 & 91.8 & 0.868 & 0.0260 & 0.457 & 0.01420 & 0.892 & 0.968 & 0.0180 & 0.0150 \\
\hline DV98-157 & 82A-7 Well & $10 / 23 / 98$ & LANL & --- & 0.154 & 623 & 99.8 & 91.7 & 1.183 & 0.0164 & 0.749 & 0.02149 & 1.467 & 0.656 & 0.0051 & 0.0409 \\
\hline \multicolumn{17}{|c|}{ Average 1998 Geothermometer $(n=19)$} \\
\hline DV99-183 & 76A-7 Well & 05/04/99 & LANL & --- & 0.152 & 438 & 99.8 & 96.3 & 0.604 & 0.0110 & 0.914 & 0.0314 & 1.208 & 0.557 & 0.0029 & 0.0227 \\
\hline DV99-185 & 74-7 Well & $05 / 04 / 99$ & LANL & --- & 0.160 & 383 & 99.7 & 96.6 & 0.544 & 0.0115 & 0.554 & 0.0198 & 0.898 & 0.471 & 0.0027 & 0.0169 \\
\hline DV99-187 & V102+ V103 Separator & $05 / 04 / 99$ & LANL & --- & 0.137 & 629 & 99.8 & 95.8 & 0.694 & 0.0118 & 0.860 & 0.0305 & 1.600 & 0.788 & 0.0243 & 0.0297 \\
\hline DV99-189 & 63-7 Well & $05 / 04 / 99$ & LANL & & 0.152 & 459 & 99.8 & 97.1 & 0.494 & 0.0205 & 0.574 & 0.0197 & 1.070 & 0.620 & 0.0257 & 0.0195 \\
\hline
\end{tabular}


Table 8: Continued

\begin{tabular}{|c|c|c|c|c|c|c|c|c|c|c|c|c|c|c|c|c|}
\hline Sample & Name or Description & Date & Laboratory & $\begin{array}{l}\text { Sampling } \\
\text { Temp. }\left({ }^{\circ} \mathrm{C}\right)\end{array}$ & $\begin{array}{c}\text { Steam } \\
\text { Fraction }(y)\end{array}$ & $\begin{array}{c}\text { Steam/Gas } \\
\text { (molar) }\end{array}$ & $\begin{array}{c}\mathrm{H}_{2} \mathrm{O} \\
\text { (mol\%, wet) }\end{array}$ & $\mathrm{CO}_{2}$ & $\mathbf{H}_{2} \mathbf{S}$ & $\mathbf{H}_{2}$ & $\mathrm{CH}_{4}$ & $\mathrm{C}_{2} \mathrm{H}_{6}$ & $\mathbf{N}_{2}$ & $\mathbf{N H}_{3}$ & $\mathrm{O}_{2}$ & Ar \\
\hline DV99-193 & V105 Separator & $05 / 05 / 99$ & LANL & --- & --- & 536 & 99.8 & 95.8 & 0.817 & 0.0180 & 0.724 & 0.0224 & 1.419 & 1.004 & 0.0140 & 0.0240 \\
\hline DV99-203 & V101 Separator & $05 / 05 / 99$ & LANL & --- & --- & 400 & 99.7 & 96.2 & 0.916 & 0.0150 & 0.466 & 0.0087 & 1.535 & 0.735 & 0.0120 & 0.0270 \\
\hline DV99-191 & 73-7 Well & $05 / 04 / 99$ & LANL & --- & 0.154 & 471 & 99.8 & 94.7 & 0.825 & 0.0143 & 0.300 & 0.0102 & 0.611 & 0.646 & 0.0194 & 0.0109 \\
\hline DV99-192 & 73B-7 Well & $05 / 04 / 99$ & LANL & --- & 0.159 & 812 & 99.9 & 86.9 & 1.714 & 0.0333 & 0.544 & 0.0167 & 1.097 & 0.700 & 0.0171 & 0.0363 \\
\hline DV99-195 & $82 \mathrm{~A}-7$ Well & 05/05/99 & LANL & --- & 0.152 & 475 & 99.8 & 93.4 & 1.128 & 0.0091 & 0.426 & 0.0127 & 0.825 & 0.418 & 0.0058 & 0.0269 \\
\hline DV99-201 & 28-33 Well & $05 / 05 / 99$ & LANL & --- & 0.159 & 257 & 99.6 & 97.8 & 0.596 & 0.0268 & 0.253 & 0.0044 & 0.909 & 0.311 & 0.0072 & 0.0231 \\
\hline DV99-202 & 37-33 Well & $05 / 05 / 99$ & LANL & -- & 0.160 & 229 & 99.6 & 97.2 & 0.597 & 0.0258 & 0.407 & 0.0093 & 1.250 & 0.302 & 0.0164 & 0.0263 \\
\hline \multicolumn{17}{|c|}{ Average 1999 Geothermometer $(n=11)$} \\
\hline \multicolumn{17}{|c|}{ Other Geothermal Wells } \\
\hline DV97-53 & 46-32 Well & $11 / 05 / 97$ & LANL & 155 & --- & 27.7 & 96.5 & 97.8 & 0.357 & 1.454 & 0.085 & 0.00109 & 0.229 & 0.046 & 0.0024 & 0.0060 \\
\hline DV97-53 & 46-32 Well & $11 / 05 / 97$ & USGS & 155 & --- & 21.0 & 95.5 & 97.4 & 0.333 & 1.866 & 0.102 & --- & 0.285 & 0.038 & 0.0000 & 0.0076 \\
\hline DV97-54 & $27-32$ Well & $11 / 05 / 97$ & USGS & 144 & 0.054 & 454 & 99.8 & 97.1 & 0.378 & 0.2884 & 0.264 & --- & 1.171 & 0.782 & 0.0349 & 0.0263 \\
\hline DV98-99 & 27-32 Well & $04 / 29 / 98$ & USGS & --- & --- & 588 & 99.8 & 95.9 & 1.452 & 0.5163 & 0.247 & --- & 0.819 & 0.981 & 0.0000 & 0.0197 \\
\hline DV98-102 & 45-14 Well & $04 / 30 / 98$ & USGS & 123.5 & --- & 47.0 & 97.9 & 67.8 & 1.181 & 0.5455 & 2.11 & --- & 26.1 & 0.079 & 1.72 & 0.4685 \\
\hline DV98-111 & $62-21$ Well & $04 / 30 / 98$ & USGS & 75.5 & --- & --- & --- & 77.1 & 0.0035 & 0.1769 & 1.92 & 0.0153 & 20.1 & --- & 0.298 & 0.3610 \\
\hline \multicolumn{17}{|l|}{ Hot Springs } \\
\hline DV98-112 & Hyder Hot Spring & $04 / 30 / 98$ & LANL & 75.3 & --- & 2.51 & 71.5 & 94.6 & $<0.004$ & $<0.0006$ & 0.536 & 0.00052 & 4.24 & $<0.0006$ & 0.2462 & 0.1252 \\
\hline DV98-117 & Sou Hot Spring & $05 / 04 / 98$ & LANL & 72.0 & --- & 1.76 & 63.8 & 52.0 & $<0.02$ & $<0.005$ & 0.801 & $<0.005$ & 46.1 & $<0.003$ & 0.5132 & 0.8394 \\
\hline \multicolumn{17}{|c|}{ Fumaroles, Senator Fumarole and Dead Zone Areas } \\
\hline DV97-43 & Crack 4 Fumarole & $11 / 03 / 97$ & USGS & 97.6 & -.- & 225 & 99.6 & 94.4 & 0.754 & 1.231 & 0.111 & & 3.15 & 0.112 & 0.180 & 0.0725 \\
\hline DV97-44 & Senator Fumarole & $11 / 03 / 97$ & LANL & 97.3 & $\cdots$ & 412 & 99.8 & 91.8 & 1.274 & 0.1753 & 0.125 & 0.00053 & 4.08 & 1.501 & 0.8473 & 0.0810 \\
\hline DV97-44 & Senator Fumarole & $11 / 03 / 97$ & USGS & 97.3 & $\cdots$ & 420 & 99.8 & 88.8 & 1.883 & 0.2310 & 0.144 & -- & 5.39 & 2.389 & 1.1 .38 & 0.121 \\
\hline DV97-45 & Range Front Fumarole & $11 / 03 / 97$ & USGS & 97.3 & --- & 357 & 99.7 & 92.3 & 1.931 & 0.2230 & 0.147 & --- & 5.25 & 0.014 & 0.0000 & 0.119 \\
\hline DV98-108 & Senator Fumarole & $05 / 01 / 98$ & USGS & 97.1 & --- & 345 & 99.7 & 96.6 & 0.579 & 0.3005 & 0.204 & --- & 1.406 & 0.888 & 0.0000 & 0.0335 \\
\hline DV98-108 & Senator Fumarole & $05 / 01 / 98$ & LANL & 97.1 & --- & 289 & 99.7 & 96.5 & 0.515 & 0.3361 & 0.234 & 0.00173 & 1.824 & 0.139 & 0.0339 & 0.0393 \\
\hline DV98-109 & Calcite Fumarole & $05 / 01 / 98$ & USGS & 95.8 & --- & 258 & 99.6 & 96.0 & 0.739 & 0.6738 & 0.102 & --- & 0.862 & 1.595 & 0.0007 & 0.0214 \\
\hline DV98-124 & Crack 4 Fumarole & $05 / 06 / 98$ & USGS & 97.6 & --- & 119 & 98.8 & 90.9 & 0.406 & 1.1680 & 0.165 & --- & 6.771 & 0.00746 & 0.4518 & 0.0969 \\
\hline DV98-164 & Senator Fumarole & $10 / 24 / 98$ & LANL & 96.5 & --- & 471 & 99.8 & 91.0 & 0.574 & 0.1591 & 0.192 & 0.00292 & 5.42 & 1.023 & 1.4601 & 0.112 \\
\hline DV98-164 & Senator Fumarole & $10 / 24 / 98$ & USGS & 96.5 & --- & --- & --- & 88.6 & 0.0084 & 0.2461 & 0.224 & 0.0038 & 8.01 & --- & 2.796 & 0.159 \\
\hline DV $98-165$ & Calcite Fumarole & $10 / 25 / 98$ & LANL & 97.0 & $\cdots$ & 265 & 99.6 & 94.0 & 0.718 & 0.4084 & 0.104 & 0.00137 & 2.69 & 1.428 & 0.2025 & 0.0515 \\
\hline DV98-166 & South Bench Fumarole & $10 / 26 / 98$ & LANL & 97.4 & --- & 407 & 99.8 & 92.1 & 1.035 & 0.2251 & 0.209 & 0.00262 & 5.21 & 0.017 & 0.8531 & 0.104 \\
\hline DV98-167 & Lonely Fumarole & $10 / 26 / 98$ & LANL & 96.4 & --- & 29.7 & 96.7 & 97.2 & 0.417 & 0.5530 & 0.136 & 0.00215 & 1.62 & $<0.0004$ & 0.0546 & 0.0270 \\
\hline DV98-181" & Figure 8 Fumarole & $10 / 29 / 98$ & LANL & 97.4 & --- & 3.19 & 76.1 & 31.1 & 0.069 & 0.0895 & $<0.007$ & $<0.007$ & 53.6 & 0.012 & 14.7 & 0.647 \\
\hline \multicolumn{17}{|c|}{ Average Geothermometer $(n=12)$} \\
\hline \multicolumn{17}{|c|}{ Fumaroles, Southwest of Cottonwood Canyon Near Inactive Sinter Deposits } \\
\hline DV98-105 & Unnamed Fumarole \#1 & $04 / 30 / 98$ & LANL & 99.5 & $\cdots$ & 16.4 & 94.2 & 3.3 .5 & $<0.004$ & 0.0263 & 0.132 & $<0.007$ & 52.4 & 0.004 & 13.1 & 0.639 \\
\hline DV99-212 & Unnamed Fumarole $\# 2$ & $05 / 10 / 99$ & LANL & 96.9 & & 26.9 & 96.4 & 32.2 & 0.104 & $<0.006$ & 0.000 & $<0.006$ & 49.1 & 0.019 & 12.6 & 0.519 \\
\hline
\end{tabular}

"CO- $\mathrm{CH}_{2} \mathrm{~S}-\mathrm{H}_{2}-\mathrm{CH}_{4}$ geothermoneter equations of D'Amore and Panichi (1980).

${ }^{\mathrm{C}} \mathrm{CO}_{2}-\mathrm{CH}_{4}$ geothermometer equation of Norman and Bernhardt (1981).

$\mathrm{H}_{2}$-Ar geothermometer equation of Giggenbach (1992): parentheses mean value is ignored for averaging.

"Two samples were mistakenly labeled 181; DV98-181 (Figure 8 Fumarole) and DV99-181 (Goerenger Well). 
Table 8: Continued

Sample He (as $\mathrm{HCl}$ ) (as $\mathrm{HF}$ )

Production and On-Site Wells

DIXE102G 0

DIXE102G

DV96-7a $\quad 0.00584 \quad 0.0$

DV96-7b

$\begin{array}{ll}0.00584 & 0.0 \\ 0.00219 & 0.0\end{array}$

DV96-7c

0.00307

0.0

DV96-10b

0.00226

0.0

Average 1996 Geothermometer $(n=5)$

DV97-12 (2) $0.00215-0.0026-0.319 \quad 0.133 \quad 5.30 \mathrm{E}-04$

$\begin{array}{llcccc}\text { DV97-12 (2) } & 0.00215 & --- & --- & --- & -- \\ \text { DV97-15 } & 0.00261 & 0.319 & 0.113 & 5.30 \mathrm{E}-04 & 4.18 \mathrm{E}-05\end{array}$

$\begin{array}{ccccc}0.00261 & 0.319 & 0.113 & 5.30 \mathrm{E}-04 & 4.18 \mathrm{E}-05 \\ 0.00219 & -- & --- & --- & ---\end{array}$

DV97-17 0.00196 $<0.03<0.04 \quad 6.94 \mathrm{E}-05 \quad 7.94 \mathrm{E}-05$ 0.00219

0.00216

$--\quad-$

DV97-

0.00216

DV97-21 (1) 0.00209

DV97-22

0.00182

$---$

DV97-24

0.00198

$---$

DV97-28

DV97-31

0.00000

$--$

Average 1997 Geothermometer $(n=11)$

$\begin{array}{llllll}\text { DV98-74 } & 0.00220 & 0.0445 & <0.02 & 1.04 \mathrm{E}-04 & 4.53 \mathrm{E}-05\end{array}$ 0.00260

0.00222

$---$

0.00241

$0.00270-0.0330-0.441$

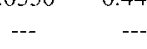

DV $98-83$

DV98-85 0.0023

0.00225

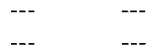

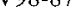

0.00217

0.00215

DV98-94

DV98-137

0.00209

$----$

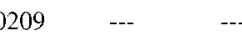

DV98-139

DV98-142

$0.00258 \quad 0.0134<0.02<-7.24 \mathrm{E}-05 \quad 3.94 \mathrm{E}-05$

$<0.0002 \quad 0.1370<<0.3$

$<.0002-0.1370<0.3-1.52 \mathrm{E}-04-8.01 \mathrm{E}-05$

$\begin{array}{lllll}0.00279 & 0.0642 & 0.039 & 4.71 \mathrm{E}-05 & 8.40 \mathrm{E}-05 \\ 0.00212 & 0.224 & <0.02 & 2.69 \mathrm{E}-03 & 4.03 \mathrm{E}-05\end{array}$

$\begin{array}{lllll}0.00212 & 0.224 & <0.02 & 2.69 \mathrm{E}-03 & 4.03 \mathrm{E}-05\end{array}$

$\begin{array}{llllll}\text { DV98-149 } & 0.00110 & 0.211 & 2.060 & 1.06 \mathrm{E}-04 & 2.19 \mathrm{E}-04 \\ \text { DV98-153 } & 0.00110 & 2.520 & 2.440 & 2.17 \mathrm{E}-03 & 9.33 \mathrm{E}-05\end{array}$

$\begin{array}{llllll}\text { DV98-157 } & 0.00416 & 3.78 & 0.410 & 2.17 \mathrm{E}-05 & 1.52 \mathrm{E}-04\end{array}$

Average 1998 Geothermometer $(n=19)$

$\begin{array}{llllll}\text { DV99-183 } & 0.0018 & 0.269 & 0.0827 & 1.01 \mathrm{E}-05 & 3.85 \mathrm{E}-05\end{array}$

$\begin{array}{lllllll}\text { DV99-185 } & 0.0017 & 0.860 & 0.0403 & 6.71 \mathrm{E}-04 & 1.08 \mathrm{E}-04\end{array}$

$\begin{array}{llllll}\text { DV99-187 } & 0.0023 & 0.0999 & 0.0245 & 6.75 \mathrm{E}-05 & 8.98 \mathrm{E}-05 \\ \text { DV99-189 } & 0.0016 & 0.0508 & 0.0293 & 2.12 \mathrm{E}-0.5 & 5.81 \mathrm{E}-05\end{array}$
Total $\delta^{13} \mathrm{C}-\mathrm{CO} \quad$ Lab for ${ }^{13} \mathrm{C} \quad$ T D-Pa $\quad \mathrm{T} \mathrm{CO}_{2}-\mathrm{CH}_{4}^{\mathrm{b}} \quad \mathrm{T} \mathrm{H}_{2} / \mathrm{Ar}^{\mathrm{c}} \quad$ Comments

(per mil)

$\left({ }^{\circ} \mathrm{C}\right)$

$\left({ }^{\circ} \mathrm{C}\right)$

$\left({ }^{\circ} \mathrm{C}\right)$

$\mathrm{H}_{2} \mathrm{~S}$ analysis may be suspect

High $\mathrm{N}_{\text {, indicates air contamination? }}$

Corrected for air contamination

Miniseparator; air contamination 
Table 8: Continued

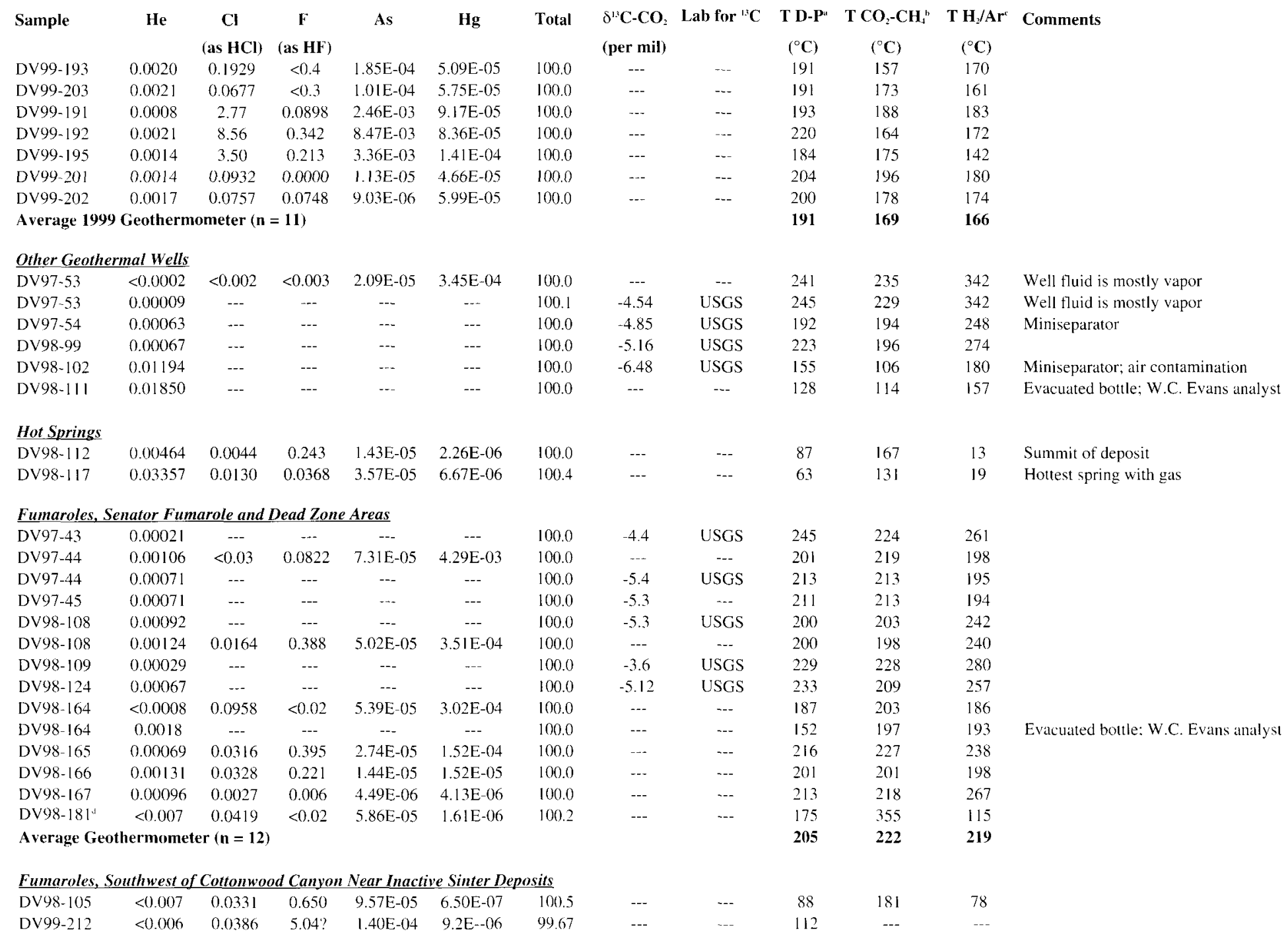


Table 9: Trace Metal Analyses of Selected Dixie Valley Region Gas Samples (Values are in ppm unless otherwise noted).a

\begin{tabular}{|c|c|c|c|c|c|c|c|c|c|c|c|}
\hline Sample & Name or Description & Ag & As & Au & $\mathrm{Cu}$ & Hg & $\mathbf{P b}$ & Sb & Se & $\mathbf{Z n}$ & $\begin{array}{r}\text { Density } \\
\left(\mathrm{g} / \mathrm{cm}^{3}\right)\end{array}$ \\
\hline \multicolumn{12}{|c|}{ Production Wells } \\
\hline DV97-12(2) & 73-7 Well & $<0.01$ & 0.205 & $<0.01$ & 0.02 & 0.012 & 0.07 & 0.027 & $<0.0002$ & 0.23 & 1.0719 \\
\hline DV97-15(2) & 74-7 Well & $<0.01$ & 0.111 & $<0.01$ & 0.02 & 0.010 & $<0.02$ & 0.010 & $<0.0002$ & 0.23 & 1.0783 \\
\hline DV97-17 & V102+V103 Separator & $<0.01$ & 0.020 & $<0.01$ & 0.38 & 0.0039 & $<0.02$ & 0.0038 & $<0.0002$ & 0.21 & 1.0748 \\
\hline DV97-19 & V105 Separator & 0.050 & 0.007 & $<0.01$ & 0.21 & 0.011 & $<0.02$ & 0.0061 & $<0.0002$ & 0.24 & 1.0758 \\
\hline DV97-21(1) & $82 \mathrm{~A}-7$ Well & $<0.01$ & 0.084 & $<0.01$ & 0.04 & 0.020 & $<0.02$ & 0.0087 & $<0.0002$ & 0.18 & 1.0436 \\
\hline DV97-21(2) & 82A-7 Well & $<0.01$ & 0.215 & $<0.01$ & 0.02 & 0.020 & $<0.02$ & 0.032 & $<0.0002$ & 0.15 & 1.0681 \\
\hline DV97-22 & 73B-7 Well & $<0.01$ & 0.079 & $<0.01$ & $<0.01$ & 0.012 & $<0.02$ & 0.018 & $<0.0002$ & 0.17 & 1.0820 \\
\hline DV97-24 & V101 Separator & $<0.01$ & 0.008 & $<0.01$ & 0.24 & 0.0035 & $<0.02$ & 0.0094 & 0.0005 & 0.27 & 1.0660 \\
\hline DV97-27 & 27-33 Well & $<0.01$ & 0.008 & $<0.01$ & 0.03 & 0.0056 & $<0.02$ & 0.0062 & $<0.0002$ & 0.21 & 1.0888 \\
\hline DV97-28 & 37-33 Well & $<0.01$ & 0.007 & $<0.01$ & $<0.01$ & 0.0070 & $<0.02$ & 0.0064 & 0.0003 & 0.18 & 1.0942 \\
\hline DV97-31 & 28-33 Well & $<0.01$ & 0.078 & $<0.01$ & 0.13 & 0.013 & 0.16 & 0.11 & 0.0007 & 0.61 & 1.1151 \\
\hline \multicolumn{12}{|c|}{ Other Geothermal Wells } \\
\hline DV97-53 & 46-32 Well & $<0.01$ & 0.014 & $<0.01$ & 0.02 & 0.56 & $<0.02$ & 0.0084 & $<0.0002$ & 0.24 & 1.1247 \\
\hline DV97-54 & 27-32 well & $<0.01$ & 0.010 & $<0.01$ & 0.03 & 0.067 & $<0.02$ & 0.010 & $<0.0002$ & 0.16 & 1.0815 \\
\hline DV98-102 & 45-14 Well & $<0.01$ & 0.037 & $<0.01$ & 0.03 & 0.20 & $<0.02$ & 0.012 & 0.0005 & 0.02 & 1.1245 \\
\hline \multicolumn{12}{|l|}{ Fumaroles } \\
\hline DV97-43 & Crack 4 Fumarole & $<0.01$ & 0.008 & $<0.01$ & $<0.01$ & 0.11 & $<0.02$ & 0.0076 & $<0.0002$ & 0.17 & 1.0871 \\
\hline DV97-44 & Senator Fumarole & $<0.01$ & 0.004 & $<0.01$ & 0.02 & 0.18 & $<0.02$ & 0.0062 & 0.0004 & 0.29 & 1.0778 \\
\hline DV $97-45$ & Range Front Fumarole & $<0.01$ & 0.009 & $<0.01$ & $<0.01$ & 0.054 & $<0.02$ & 0.0065 & 0.0005 & 0.24 & 1.0830 \\
\hline DV98-108 & Senator Fumarole & $<0.01$ & 0.009 & $<0.01$ & $<0.01$ & 0.021 & $<0.02$ & 0.0075 & $<0.0002$ & 0.18 & 1.0844 \\
\hline DV98-109 & Calcite Fumarole & $<0.01$ & 0.014 & $<0.01$ & 0.02 & 0.028 & 0.03 & 0.023 & $<0.0002$ & 0.18 & 1.0821 \\
\hline DV98-124 & Crack 4 Fumarole & $<0.01$ & 0.009 & $<0.01$ & $<0.01$ & 0.023 & $<0.02$ & 0.0090 & $<0.0002$ & 0.06 & 1.1160 \\
\hline
\end{tabular}

"Analyses performed on caustic solutions obtained from USGS samples (Table 8). Values are corrected for density and background (blank sample). 
Table 10: Reconstructed Reservoir Compositions Used For Geothermometry Calculations (Values in ppm except where otherwise noted)."

\begin{tabular}{|c|c|c|c|c|c|c|c|c|c|c|c|c|c|c|c|c|c|}
\hline \multirow[t]{2}{*}{ Sample } & \multirow[t]{2}{*}{ Location } & \multirow[t]{2}{*}{ Date } & \multirow[t]{2}{*}{$\mathrm{y}$ value } & \multicolumn{7}{|c|}{ Brine Analyses } & \multicolumn{7}{|c|}{ Reconstructed Reservoir Compositions } \\
\hline & & & & $\mathrm{Cl}$ & $\mathrm{SiO}_{2}$ & $\mathbf{C a}$ & $\mathbf{M g}^{\circ}$ & $\mathrm{Na}$ & $\mathbf{K}$ & $\mathbf{L i}$ & $\mathrm{Cl}$ & $\mathrm{SiO}_{2}$ & $\mathrm{Ca}$ & $\mathrm{Mg}$ & $\mathbf{N a}$ & $\mathrm{K}$ & $\mathrm{Li}$ \\
\hline DIXE102-W & $V 102+V 103$ Separator & $10 / 02 / 95$ & 0.153 & 495 & 638.0 & 7.92 & 0.04 & 462 & 71.8 & 2.29 & 419.3 & 540.4 & 6.71 & 0.034 & 391.3 & 60.8 & 1.94 \\
\hline DV96-8 & 76-7 Well & $10 / 25 / 96$ & 0.184 & 524 & 599.0 & 8.53 & 0.026 & 474 & 69.5 & 2.29 & 427.6 & 488.8 & 6.96 & 0.021 & 386.8 & 56.7 & 1.87 \\
\hline DV96-9 & V101 Separator & $10 / 25 / 96$ & 0.159 & 438 & 599.0 & 8.03 & 0.007 & 407 & 64 & 2.03 & 368.4 & 503.8 & 6.75 & 0.006 & 342.3 & 53.8 & 1.71 \\
\hline DV97-11 & V101 Separator & $10 / 29 / 97$ & 0.158 & 594 & 580.0 & 8.96 & 0.02 & 508 & 74.4 & 2.45 & 500.1 & 488.4 & 7.54 & 0.017 & 427.7 & 62.6 & 2.06 \\
\hline DV97-13 & 84-7 Well & 10/29/97 & 0.159 & 558 & 580.0 & 9.66 & 0.01 & 496 & 70.8 & 2.46 & 469.3 & 487.8 & 8.12 & 0.008 & 417.1 & 59.5 & 2.07 \\
\hline DV97-14 & 74-7 Well & $10 / 29 / 97$ & 0.163 & 584 & 586.0 & 9.2 & 0.01 & 500 & 72.2 & 2.43 & 488.8 & 490.5 & 7.70 & 0.008 & 418.5 & 60.4 & 2.03 \\
\hline DV97-16 & V102 + V103 Separator & $10 / 29 / 97$ & 0.161 & 580 & 586.0 & 9.02 & 0.01 & 500 & 77.2 & 2.48 & 486.6 & 491.7 & 7.57 & 0.008 & 419.5 & 64.8 & 2.08 \\
\hline DV97-18 & V105 Separator & $10 / 29 / 97$ & 0.151 & 574 & 595.0 & 9.53 & 0.02 & 502 & 73.5 & 2.29 & 487.3 & 505.2 & 8.09 & 0.017 & 426.2 & 62.4 & 1.94 \\
\hline DV97-20 & 82A-7 Well & $10 / 29 / 97$ & 0.159 & 575 & 556.0 & 9.63 & 0.01 & 495 & 72.6 & 2.22 & 483.6 & 467.6 & 8.10 & 0.008 & 416.3 & 61.1 & 1.87 \\
\hline DV97-23 & 73B-7 Well & $10 / 30 / 97$ & 0.160 & 571 & 569.0 & 9.09 & 0.01 & 499 & 76.4 & 2.34 & 479.6 & 478.0 & 7.64 & 0.008 & 419.2 & 64.2 & 1.97 \\
\hline DV97-25 & 27-33 Well & $10 / 30 / 97$ & 0.157 & 443 & 627.0 & 7.69 & 0.01 & 423 & 66.8 & 2.22 & 373.4 & 528.6 & 6.48 & 0.008 & 356.6 & 56.3 & 1.87 \\
\hline DV97-26 & V101 Separator & $10 / 30 / 97$ & 0.164 & 463 & 627.0 & 7.95 & 0.01 & 439 & 68.8 & 2.27 & 387.1 & 524.2 & 6.65 & 0.008 & 367.0 & 57.5 & 1.90 \\
\hline DV97-29 & $37-33$ Well & $10 / 30 / 97$ & 0.159 & 475 & 621.0 & 7.2 & 0.02 & 431 & 68.8 & 2.26 & 399.5 & 522.3 & 6.06 & 0.017 & 362.5 & 57.9 & 1.90 \\
\hline DV $97-30$ & $28-33$ Well & $10 / 30 / 97$ & 0.156 & 470 & 642.0 & 7.4 & 0.02 & 429 & 70.1 & 2.24 & 396.7 & 541.8 & 6.25 & 0.017 & 362.1 & 59.2 & 1.89 \\
\hline DV98-73 & V101 Separator & $04 / 28 / 98$ & 0.157 & 449 & 590.6 & 7.41 & 0.05 & 448 & 70.5 & 2.40 & 378.5 & 497.9 & 6.25 & 0.042 & 377.8 & 59.4 & 2.02 \\
\hline DV98-75 & 27-33 Well & $04 / 28 / 98$ & 0.155 & $42]$ & 571.4 & 6.97 & 0.01 & 430 & 60.2 & 2.27 & 355.7 & 482.8 & 5.89 & 0.008 & 363.4 & 50.9 & 1.92 \\
\hline DV98-77 & 37-33 Well & $04 / 28 / 98$ & 0.156 & 444 & 554.3 & 7.19 & 0.01 & 429 & 66.7 & 2.21 & 374.7 & 467.8 & 6.07 & 0.008 & 362.1 & 56.3 & 1.87 \\
\hline DV98-79 & 28-33 Well & $04 / 28 / 98$ & 0.157 & 446 & 550.0 & 7.50 & 0.01 & 447 & 67.8 & 2.28 & 376.0 & 463.6 & 6.32 & 0.008 & 376.4 & 57.2 & 1.92 \\
\hline DV98-80 & 76A-7 Well & $04 / 28 / 98$ & 0.157 & 556 & 541.4 & 8.56 & 0.01 & 498 & 75.6 & 2.58 & 468.7 & 456.4 & 7.22 & 0.008 & 419.8 & 63.7 & 2.17 \\
\hline DV $98-82$ & V102+V103 Separator & $04 / 28 / 98$ & 0.150 & 567 & 517.9 & 8.81 & 0.01 & 498 & 77.1 & 2.42 & 482.0 & 440.2 & 7.49 & 0.009 & 423.3 & 65.5 & 2.06 \\
\hline DV98-84 & 74-7 Well & $04 / 28 / 98$ & 0.158 & 564 & 530.7 & 8.65 & 0.02 & 491 & 75.2 & 253 & 474.9 & 446.9 & 7.28 & 0.017 & 413.7 & 63.3 & 2.13 \\
\hline DV98-86 & 63-7 Well & $04 / 28 / 98$ & 0.154 & 560 & 515.7 & 8.73 & 0.01 & 510 & 77.0 & 2.43 & 473.8 & 436.3 & 7.39 & 0.008 & 431.1 & 65.1 & 2.06 \\
\hline DV98-88 & 73-7 Well & $04 / 29 / 98$ & 0.154 & 547 & 517.9 & 8.44 & 0.02 & 498 & 76.8 & 2.40 & 462.8 & 438.1 & 7.14 & 0.017 & 421.3 & 65.0 & 2.03 \\
\hline DV98-90 & $82 \mathrm{~A}-7 \mathrm{Well}$ & $04 / 29 / 98$ & 0.153 & 561 & 520.0 & 8.95 & 0.01 & 501 & 76.1 & 2.22 & 475.2 & 440.5 & 7.58 & 0.008 & 424.6 & 64.5 & 1.88 \\
\hline DV98-92 & V105 Separator & $04 / 29 / 98$ & 0.150 & 572 & 526.4 & 8.65 & 0.01 & 496 & 75.9 & 2.32 & 486.2 & 447.5 & 7.35 & 0.009 & 421.9 & 64.5 & 1.97 \\
\hline DV98-95 & 73B-7 Well & $04 / 29 / 98$ & 0.152 & 561 & 511.5 & 8.43 & 0.01 & 500 & 74.2 & 2.27 & 475.7 & 433.7 & 7.15 & 0.008 & 423.7 & 62.9 & 1.92 \\
\hline DV98-135 & $27-33$ Well & $10 / 20 / 98$ & 0.184 & 496 & 582.1 & 9.46 & 0.01 & 467 & 60.0 & 2.61 & 404.7 & 475.0 & 7.72 & 0.008 & 381.1 & 49.0 & 2.13 \\
\hline DV98-138 & V101 Separator & $10 / 21 / 98$ & 0.160 & 436 & 545.7 & 7.18 & 0.01 & 409 & 63.8 & 2.11 & 366.2 & 458.4 & 6.03 & 0.008 & 343.6 & 53.6 & 1.77 \\
\hline DV98- 140 & $37-33$ Well & $10 / 21 / 98$ & 0.162 & 432 & 526.4 & 6.08 & 0.21 & 398 & 66.3 & 2.02 & 362.0 & 441.2 & 5.10 & 0.176 & 333.5 & 55.6 & 1.69 \\
\hline DV98-141 & 28-33 Well & $10 / 21 / 98$ & 0.162 & 441 & 530.7 & 7.21 & 0.03 & 412 & 65.5 & 2.03 & 369.6 & 444.7 & 6.04 & 0.025 & 34.5 .3 & 54.9 & 1.70 \\
\hline DV98-145 & 76A-7 Well & $10 / 22 / 98$ & 0.158 & 541 & 498.6 & 8.23 & 0.01 & 479 & 70.8 & 2.26 & 455.5 & 419.8 & 6.93 & 0.008 & 403.3 & 59.6 & 1.90 \\
\hline DV98-147 & 63-7 Well & $10 / 22 / 98$ & 0.155 & 565 & 500.8 & 8.87 & 0.01 & 496 & 71.9 & 2.21 & 477.4 & 423.1 & 7.50 & 0.008 & 419.1 & 60.8 & 1.87 \\
\hline DV98-148 & V $102+$ V 103 Separator & $10 / 22 / 98$ & 0.164 & 560 & 513.6 & 9.18 & 0.17 & 485 & 72.3 & 2.24 & 468.2 & 429.4 & 7.67 & 0.142 & 405.5 & 60.4 & 1.87 \\
\hline DV $98-150$ & 74-7 Well & $10 / 22 / 98$ & 0.160 & 554 & 517.9 & 8.90 & 0.04 & 486 & 74.1 & 2.38 & 465.4 & 435.0 & 7.48 & 0.034 & 408.2 & 62.2 & 2.00 \\
\hline DV98-1.52 & 73-7 Well & $10 / 22 / 98$ & 0.154 & 567 & 509.3 & 8.81 & 0.02 & 476 & 73.7 & 2.14 & 479.7 & 430.9 & 7.45 & 0.017 & 402.7 & 62.4 & 1.81 \\
\hline DV98- 1.54 & 73B-7 Well & $10 / 22 / 98$ & 0.154 & 560 & 513.6 & 7.75 & 0.01 & 485 & 72.0 & 2.15 & 473.8 & 434.5 & 6.56 & 0.008 & 410.3 & 60.9 & 1.82 \\
\hline DV $98-156$ & 82A-7 Well & $10 / 23 / 98$ & 0.154 & 557 & 513.6 & 8.87 & 0.04 & 473 & 69.3 & 2.10 & 471.2 & 4.34 .5 & 7.50 & 0.034 & 400.2 & 58.6 & 1.78 \\
\hline DV98-159 & V105 Separator & $10 / 23 / 98$ & 0.146 & 560 & 507.2 & 9.39 & 0.34 & 480 & 69.4 & 2.14 & 478.2 & 433.1 & 8.02 & 0.290 & 409.9 & 59.3 & 1.83 \\
\hline DV99-182 & $76 \mathrm{~A}-7 \mathrm{Well}$ & $05 / 04 / 99$ & 0.152 & 576 & 524 & 8.52 & 0.24 & 508 & 73.7 & 2.51 & 488.4 & 444.6 & 7.22 & 0.204 & 430.8 & 62.5 & 2.13 \\
\hline DV99-184 & 74-7 Well & $05 / 04 / 99$ & 0.160 & 592 & 522 & 8.65 & 0.33 & 482 & 74.3 & 2.32 & 497.3 & 438.6 & 7.27 & 0.277 & 404.9 & 62.4 & 1.95 \\
\hline DV99-186 & V $102+V 103$ Separator & $05 / 04 / 99$ & 0.137 & 594 & 522 & 8.47 & 0.01 & 496 & 72.7 & 2.33 & 512.6 & 450.6 & 7.31 & 0.009 & 428.0 & 62.7 & 2.01 \\
\hline
\end{tabular}


Table 10: Continued

\begin{tabular}{|c|c|c|c|c|c|c|c|c|c|c|c|c|c|c|c|c|c|}
\hline \multirow[t]{2}{*}{ Sample } & \multirow[t]{2}{*}{ Location } & \multirow[t]{2}{*}{ Date } & \multirow[t]{2}{*}{ y value } & \multicolumn{7}{|c|}{ Brine Analyses } & \multicolumn{7}{|c|}{ Reconstructed Reservoir Compositions } \\
\hline & & & & $\mathrm{Cl}$ & $\mathrm{SiO}_{2}$ & $\mathrm{Ca}$ & $\mathbf{M g}^{\mathrm{h}}$ & $\mathrm{Na}$ & $\mathbf{K}$ & $\mathbf{L i}$ & $\mathrm{Cl}$ & $\mathrm{SiO}_{2}$ & $\mathrm{Ca}$ & $\mathrm{Mg}$ & $\mathrm{Na}$ & $\mathrm{K}$ & $\mathrm{Li}$ \\
\hline DV99-188 & 63-7 Well & $05 / 04 / 99$ & 0.152 & 604 & 516 & 8.48 & 0.01 & 504 & 73.6 & 2.26 & 512.2 & 437.3 & 7.19 & 0.008 & 427.4 & 62.4 & 1.92 \\
\hline DV99-190 & 73-7 Well & $05 / 04 / 99$ & 0.154 & 624 & 518 & 8.79 & 0.01 & 508 & 74.6 & 2.21 & 527.9 & 438.1 & 7.44 & 0.008 & 429.8 & 63.1 & 1.87 \\
\hline DV99-194 & V 105 Separator & $05 / 05 / 99$ & 0.138 & 620 & 514 & 9.51 & 0.01 & 514 & 74.4 & 2.23 & 534.4 & 442.7 & 8.20 & 0.009 & 443.1 & 64.1 & 1.92 \\
\hline DV99-196 & $82 \mathrm{~A}-7$ Well & 05/05/99 & 0.152 & 623 & 503 & 8.89 & 0.01 & 518 & 72.2 & 2.25 & 528.3 & 426.5 & 7.54 & 0.008 & 439.3 & 61.2 & 1.91 \\
\hline DV99-197 & 73B-7 Well & $05 / 05 / 99$ & 0.159 & 624 & 520 & 8.78 & 0.01 & 516 & 74.4 & 2.22 & 524.8 & 437.3 & 7.38 & 0.008 & 434.0 & 62.6 & 1.87 \\
\hline DV99-199 & $37-33$ Well & $05 / 05 / 99$ & 0.160 & 475 & 563 & 6.66 & 0.12 & 433 & 65.7 & 2.23 & 399.0 & 472.8 & 5.59 & 0.101 & 363.7 & 55.2 & 1.87 \\
\hline DV99-200 & 28-33 Well & $05 / 05 / 99$ & 0.159 & 483 & 561 & 6.68 & 0.02 & 432 & 66.2 & 2.24 & 406.2 & 471.5 & 5.62 & 0.017 & 363.3 & 55.7 & 1.88 \\
\hline DV99-204 & V101 Separator & $05 / 05 / 99$ & 0.159 & 481 & 576 & 7.35 & 0.01 & 428 & 68.4 & 2.39 & 404.5 & 484.1 & 6.18 & 0.008 & 359.9 & 57.5 & 2.01 \\
\hline DV99-204 & V101 Separator & 05/05/99 & 0.159 & 481 & 576 & 7.35 & 0.01 & 428 & 68.4 & 2.39 & 404.5 & 484.1 & 6.18 & 0.008 & 359.9 & 57.5 & 2.01 \\
\hline DV74782786-brine 2 & 74-7 Well Archived & $08 / 27 / 86$ & 0.199 & 396 & 574 & 1.11 & 0.01 & 413 & 61.5 & 2.82 & 317.2 & 459.4 & 0.89 & 0.008 & 330.8 & 49.3 & 2.26 \\
\hline DV76781986-brine 4 & 76-7 Well Archived & $08 / 19 / 86$ & 0.187 & 402 & 563 & 1.53 & 0.01 & 403 & 54.2 & 2.79 & 326.8 & 457.6 & 1.24 & 0.008 & 327.6 & 44.1 & 2.27 \\
\hline DV453382186-brine 6 & 45-33 Well Archived & $08 / 21 / 86$ & 0.165 & 320 & 589 & 1.27 & 0.04 & 370 & 59.2 & 2.63 & 267.2 & 491.4 & 1.06 & 0.033 & 309.0 & 49.4 & 2.20 \\
\hline DV73782886-brine 8 & 73-7 Well Archived & $08 / 28 / 86$ & 0.198 & 363 & 548 & 1.24 & 0.01 & 380 & 59.2 & 2.55 & 291.1 & 439.4 & 0.99 & 0.008 & 304.8 & 47.5 & 2.05 \\
\hline No number & 28-33 Well Archived & $09 / 23 / 93$ & 0.158 & 70.1 & 101 & 15.6 & 2.08 & 228 & 6.13 & 0.35 & 59.0 & 84.9 & 13.14 & 1.751 & 192.0 & 5.2 & 0.29 \\
\hline DV97-55 & 27-32 Well & $11 / 05 / 97$ & 0.054 & 87.6 & 58.2 & 5.56 & 0.03 & 95.5 & 13.1 & 0.7 & 82.9 & 55.1 & 5.26 & 0.028 & 90.3 & 12.4 & 0.66 \\
\hline DV98-99: & 27-32 Well & $04 / 29 / 98$ & 0.054 & 84.8 & 61.2 & 5.91 & 0.91 & 88.0 & 11.6 & 0.48 & 80.2 & 57.9 & 5.59 & 0.861 & 83.2 & 11.0 & 0.45 \\
\hline
\end{tabular}

a The reconstructed composition is calculated as $\mathrm{C}(\mathrm{r})=(1-\mathrm{y}) * \mathrm{C}(\mathrm{b})$, where $\mathrm{C}(\mathrm{b})=$ analyte concentration in the brine, $\mathrm{C}(\mathrm{r})=$ reconstructed analyte composition, and $\mathrm{y}=$ the steam fraction.

I "Mg values shown in bold are "less than" values that are assumed to be $0.01 \mathrm{ppm}$ for the calculations.

"The $y$ value for DV98-99 uses the 1997 value. 
Table 11: Chemical Geothermometry of Geothermal Production Fluids (Corrected for steam flash, Table 10; all values in ${ }^{\circ} \mathrm{C}$ ). ${ }^{2}$

\begin{tabular}{|c|c|c|c|c|c|c|c|c|c|c|c|c|c|c|}
\hline \multirow[t]{2}{*}{ Sample } & \multirow[t]{2}{*}{ Name or description } & \multirow[t]{2}{*}{ Date } & \multirow[t]{2}{*}{$\mathrm{tCH}$} & \multirow[t]{2}{*}{ tQC } & \multirow[t]{2}{*}{ tNK(f) } & \multirow[t]{2}{*}{$\operatorname{tNL}(d)$} & \multirow[t]{2}{*}{$\mathbf{t L}(d)$} & \multirow[t]{2}{*}{$\mathbf{t M L}^{\mathrm{b}}$} & \multirow[t]{2}{*}{$\mathbf{t K} \mathbf{M}^{\mathrm{b}}$} & \multicolumn{5}{|c|}{ <----- Na-K-Ca------> <----- Na-K-Ca Mg correction -.--.> } \\
\hline & & & & & & & & & & $\mathrm{t}(\text { beta } 1 / 3)^{\mathrm{c}}$ & $\mathbf{t}(\mathbf{b e t a} 4 / 3)^{\mathrm{c}}$ & $\mathbf{R}^{\mathbf{h}}$ & t(beta1/3) & $t($ beta4/3) \\
\hline DV74782786-brine 2 & 74-7 Well Archived & $08 / 27 / 86$ & 235 & 245 & 254 & 221 & 185 & 308 & 248 & 252 & 348 & 0.050 & $\underline{252}$ & 348 \\
\hline DV76781986-brine 4 & 76-7 Well Archived & $08 / 19 / 86$ & 235 & 244 & 244 & 222 & 185 & 308 & 241 & $\underline{241}$ & 316 & 0.056 & $\underline{241}$ & 316 \\
\hline DV453382186-brine 6 & 45-33 Well Archived & $08 / 21 / 86$ & 243 & 251 & 261 & 225 & 184 & 256 & 212 & $\underline{254}$ & 335 & 0.208 & $\underline{254}$ & 335 \\
\hline DV73782886-brine 8 & 73-7 Well Archived & $08 / 28 / 86$ & 230 & 241 & 258 & 219 & 181 & 301 & 246 & $\underline{252}$ & 334 & 0.052 & 252 & 334 \\
\hline Average August 1986 & & & 236 & 245 & 254 & 222 & 184 & 293 & 237 & 250 & & & 250 & \\
\hline No number & 28-33 Well Archived & $09 / 23 / 93$ & 100 & 128 & 125 & 101 & 115 & 84 & 72 & 123 & $\underline{94}$ & 15.466 & 99 & $\underline{94}$ \\
\hline DIXE102-W & V102 + V103 Separator & $10 / 02 / 95$ & 254 & 260 & 258 & 189 & 179 & 248 & 222 & $\underline{235}$ & 255 & 0.147 & $\underline{235}$ & 255 \\
\hline DV96-8 & 76-7 Well & $10 / 25 / 96$ & 242 & 250 & 252 & 186 & 178 & 260 & 230 & $\underline{231}$ & 248 & 0.097 & $\underline{231}$ & 248 \\
\hline DV96-9 & V101 Separator & $10 / 25 / 96$ & 246 & 253 & 259 & 189 & 174 & 299 & 261 & $\underline{233}$ & 243 & 0.028 & $\underline{233}$ & 243 \\
\hline Average October 1996 & & & 244 & 252 & 256 & 188 & 176 & 279 & 245 & 232 & & & 232 & \\
\hline DV97-11 & 73-7 Well & $10 / 29 / 97$ & 242 & 250 & 252 & 186 & 181 & 275 & 241 & $\underline{232}$ & 253 & 0.070 & $\underline{232}$ & 253 \\
\hline DV97-13 & 84-7 Well & $10 / 29 / 97$ & 242 & 250 & 250 & 189 & 182 & 300 & 257 & 229 & 246 & 0.036 & $\underline{229}$ & 246 \\
\hline DV97-14 & 74-7 Well & $10 / 29 / 97$ & 243 & 251 & 251 & 187 & 181 & 299 & 258 & $\underline{230}$ & 249 & 0.036 & $\underline{230}$ & 249 \\
\hline DV97-16 & V102 + V103 Separator & $10 / 29 / 97$ & 243 & 251 & 257 & 189 & 182 & 300 & 261 & $\underline{235}$ & 255 & 0.034 & $\underline{235}$ & 255 \\
\hline DV97-18 & V105 Separator & $10 / 29 / 97$ & 246 & 253 & 252 & 181 & 179 & 270 & 240 & 231 & 250 & 0.070 & $\underline{231}$ & 250 \\
\hline DV97-20 & $82 \mathrm{~A}-7$ Well & $10 / 29 / 97$ & 237 & 246 & 252 & 180 & 178 & 292 & 258 & $\underline{231}$ & 247 & 0.035 & $\underline{231}$ & 247 \\
\hline DV97-23 & 73B-7 Well & $10 / 30 / 97$ & 240 & 248 & 256 & 184 & 180 & 296 & 261 & $\underline{234}$ & 254 & 0.034 & $\underline{234}$ & 254 \\
\hline DV97-25 & 27-33 Well & $10 / 30 / 97$ & 251 & 257 & 260 & 194 & 178 & 292 & 253 & $\underline{235}$ & 249 & 0.039 & $\underline{235}$ & 249 \\
\hline DV97-26 & V101 Separator & $10 / 30 / 97$ & 250 & 257 & 259 & 193 & 178 & 294 & 255 & $\underline{235}$ & 250 & 0.038 & $\underline{235}$ & 250 \\
\hline DV97-29 & 37-33 Well & $10 / 30 / 97$ & 250 & 256 & 261 & 194 & 178 & 269 & 237 & $\underline{237}$ & 254 & 0.078 & $\underline{237}$ & 254 \\
\hline DV97-30 & 28-33 Well & $10 / 30 / 97$ & 254 & 260 & 263 & 194 & 178 & 269 & 238 & $\underline{238}$ & 254 & 0.076 & $\underline{238}$ & 254 \\
\hline Average October 1997 & & & 245 & 253 & 256 & $J 88$ & 179 & 287 & 251 & 233 & & & 233 & \\
\hline DV98-73 & V101 Separator & $04 / 28 / 98$ & 244 & 252 & 259 & 196 & 181 & 244 & 215 & $\underline{236}$ & 256 & 0.189 & $\underline{236}$ & 256 \\
\hline DV98-75 & 27-33 Well & $04 / 28 / 98$ & 241 & 249 & 248 & 195 & 179 & 294 & 248 & $\underline{228}$ & 246 & 0.044 & $\underline{228}$ & 246 \\
\hline DV98-77 & 37-33 Well & $04 / 28 / 98$ & 237 & 246 & 258 & 192 & 177 & 292 & 253 & $\underline{235}$ & 252 & 0.040 & $\underline{235}$ & 252 \\
\hline DV98-79 & 28-33 Well & $04 / 28 / 98$ & 236 & 246 & 256 & 192 & 179 & 294 & 254 & $\underline{234}$ & 252 & 0.039 & $\underline{234}$ & 252 \\
\hline DV98-80 & 76A-7 Well & $04 / 28 / 98$ & 235 & 244 & 256 & 193 & 184 & 303 & 260 & $\underline{235}$ & 256 & 0.035 & $\underline{235}$ & 256 \\
\hline DV98-82 & V102 + V103 Separator & $04 / 28 / 98$ & 231 & 241 & 258 & 187 & 181 & 299 & 262 & $\underline{236}$ & 257 & 0.034 & 236 & 257 \\
\hline DV98-84 & 74-7 Well & $04 / 28 / 98$ & 232 & 242 & 256 & 192 & 183 & 277 & 241 & $\underline{235}$ & 255 & 0.070 & 235 & 255 \\
\hline DV98-86 & 63-7 Well & $04 / 28 / 98$ & 230 & 240 & 255 & 185 & 181 & 299 & 261 & 235 & 258 & 0.034 & $\underline{235}$ & 258 \\
\hline DV98-88 & 73-7 Well & $04 / 29 / 98$ & 230 & 241 & 257 & 186 & 181 & 273 & 242 & $\underline{236}$ & 258 & 0.069 & $\underline{236}$ & 258 \\
\hline DV98-90 & 82A-7 Well & $04 / 29 / 98$ & 231 & 241 & 256 & 179 & 178 & 292 & 261 & $\underline{234}$ & 255 & 0.034 & $\underline{234}$ & 255 \\
\hline DV98-92 & V105 Separator & $04 / 29 / 98$ & 232 & 242 & 256 & 183 & 180 & 296 & 261 & $\underline{235}$ & 257 & 0.035 & 235 & 257 \\
\hline DV98-95 & 73B-7 Well & $04 / 29 / 98$ & 229 & 240 & 253 & 181 & 179 & 294 & 259 & $\underline{233}$ & 256 & 0.035 & $\underline{233}$ & 256 \\
\hline Average April 1998 & & & 234 & 244 & 256 & 188 & 180 & 288 & 252 & 234 & & & 234 & \\
\hline DV98-135 & 27-33 Well & $10 / 20 / 98$ & 239 & 248 & 239 & 200 & 183 & 303 & 247 & $\underline{221}$ & 232 & 0.041 & 221 & 232 \\
\hline DV98-138 & V101 Separator & $10 / 21 / 98$ & 235 & 244 & 258 & 193 & 176 & 288 & 251 & $\underline{234}$ & 248 & 0.041 & $\underline{234}$ & 248 \\
\hline DV98-140 & 37-33 Well & $10 / 21 / 98$ & 231 & 241 & 265 & 191 & 174 & 194 & 181 & $\underline{240}$ & 258 & 0.857 & 240 & 258 \\
\hline DV98-141 & 28-33 Well & $10 / 21 / 98$ & 232 & 242 & 260 & 188 & 174 & 249 & 224 & 235 & 249 & 0.121 & $\underline{235}$ & 249 \\
\hline
\end{tabular}


Table 11: Continued

\begin{tabular}{|c|c|c|c|c|c|c|c|c|c|c|c|c|c|c|}
\hline \multirow[t]{2}{*}{ Sample } & \multirow[t]{2}{*}{ Name or description } & \multirow[t]{2}{*}{ Date } & \multirow[t]{2}{*}{$\mathrm{tCH}$} & \multirow[t]{2}{*}{ tQC } & \multirow[t]{2}{*}{ tNK(f) } & \multirow[t]{2}{*}{ tNL(d) } & \multirow[t]{2}{*}{$\mathbf{t} \mathbf{L}(\mathbf{d})$} & \multirow[t]{2}{*}{ tML' } & \multirow[t]{2}{*}{$\mathrm{tKM}^{\mathrm{b}}$} & \multicolumn{2}{|c|}{$<--\cdot---$ Na-K-Ca------> } & \multicolumn{3}{|c|}{ <----- Na-K-Ca Mg correction -----> } \\
\hline & & & & & & & & & & t(beta1/3) & $t(\text { beta } 4 / 3)^{c}$ & $\mathbf{R}^{\mathbf{b}}$ & $t(\operatorname{beta} 1 / 3)^{c}$ & $\mathrm{t}\left({\text { beta } 4 / 3)^{\mathrm{c}}}\right.$ \\
\hline DV98-145 & 76A-7 Well & $10 / 22 / 98$ & 226 & 237 & 253 & 184 & 178 & 293 & 257 & $\underline{232}$ & 253 & 0.037 & $\underline{232}$ & 253 \\
\hline DV98-147 & 63-7 Well & $10 / 22 / 98$ & 226 & 237 & 251 & 179 & 178 & 292 & 258 & $\underline{231}$ & 251 & 0.036 & $\underline{231}$ & 251 \\
\hline DV98-148 & V102 + V103 Separator & $10 / 22 / 98$ & 228 & 239 & 254 & 182 & 178 & 205 & 189 & 232 & 249 & 0.603 & $\underline{228}$ & 246 \\
\hline DV98-150 & 74-7 Well & $10 / 22 / 98$ & 229 & 240 & 256 & 188 & 180 & 250 & 223 & $\underline{234}$ & 252 & 0.140 & $\underline{234}$ & 252 \\
\hline DV98-152 & 73-7 Well & $10 / 22 / 98$ & 228 & 239 & 258 & 180 & 176 & 266 & 240 & $\underline{235}$ & 252 & 0.071 & $\underline{\underline{235}}$ & 252 \\
\hline DV98-154 & 73B-7 Well & $10 / 22 / 98$ & 229 & 240 & 253 & 179 & 177 & 290 & 258 & 234 & 257 & 0.037 & $\overline{234}$ & 257 \\
\hline DV98-156 & $82 \mathrm{~A}-7$ Well & $10 / 23 / 98$ & 229 & 240 & 252 & 179 & 176 & 242 & 220 & $\underline{231}$ & 247 & 0.148 & $\underline{231}$ & 247 \\
\hline DV98-159 & V105 Separator & $10 / 23 / 98$ & 229 & 240 & 251 & 179 & 177 & 186 & 174 & 230 & 245 & 1.231 & $\underline{230}$ & 245 \\
\hline Average October 1998 & & & 230 & $24 I$ & 254 & 185 & 177 & 255 & 227 & 232 & & & 232 & \\
\hline DV99-182 & 76A-7 Well & 05/04/99 & 232 & 242 & 251 & 189 & 183 & 202 & 183 & $\underline{232}$ & 256 & 0.847 & $\underline{232}$ & 256 \\
\hline DV99-184 & 74-7 Well & 05/04/99 & 230 & 241 & 257 & 186 & 179 & 190 & 177 & $\underline{235}$ & 254 & 1.151 & 235 & 254 \\
\hline DV99-186 & V102 + V103 Separator & 05/04/99 & 233 & 243 & 252 & 184 & 180 & 297 & 259 & $\underline{232}$ & 255 & 0.036 & 232 & 255 \\
\hline DV99-188 & 63-7 Well & 05/04/99 & 230 & 240 & 252 & 180 & 179 & 294 & 259 & $\underline{232}$ & 255 & 0.036 & $\underline{232}$ & 255 \\
\hline DV99-190 & 73-7 Well & 05/04/99 & 230 & 241 & 252 & 177 & 178 & 292 & 260 & $\underline{232}$ & 255 & 0.035 & $\underline{232}$ & 255 \\
\hline DV99-194 & V105 Separator & $05 / 05 / 99$ & 231 & 241 & 251 & 177 & 179 & 293 & 260 & $\overline{231}$ & 252 & 0.035 & $\underline{231}$ & 252 \\
\hline DV99-196 & 82A-7 Well & 05/05/99 & 227 & 238 & 247 & 177 & 178 & 293 & 258 & $\underline{229}$ & 252 & 0.036 & $\underline{229}$ & 252 \\
\hline DV99-197 & 73B-7 Well & 05/05/99 & 230 & 240 & 250 & 176 & 178 & 292 & 259 & 232 & 255 & 0.035 & $\underline{232}$ & 255 \\
\hline DV99-199 & 37-33 Well & $05 / 05 / 99$ & 238 & 247 & 256 & 192 & 178 & 214 & 193 & $\underline{234}$ & 255 & 0.488 & $\overline{234}$ & 255 \\
\hline DV99-200 & 28-33 Well & 05/05/99 & 238 & 247 & 257 & 193 & 178 & 268 & 235 & $\underline{235}$ & 255 & $0.08 \mathrm{l}$ & $\underline{235}$ & 255 \\
\hline DV99-204 & V101 Separator & 05/05/99 & 241 & 249 & 261 & 200 & 180 & 298 & 255 & $\overline{236}$ & 253 & 0.039 & $\underline{236}$ & 253 \\
\hline Average May 1999 & & & 233 & 243 & 253 & 185 & 179 & 267 & 236 & $\overline{233}$ & & & $\overline{233}$ & \\
\hline DV97-55 & 27-32 Monitoring Well & I l $/ 05 / 97$ & 76 & 106 & 246 & 228 & 140 & 193 & 158 & 199 & 144 & 0.401 & 199 & 144 \\
\hline DV98-99 & 27-32 Monitoring Well & 04/29/98 & 79 & 109 & 242 & 198 & 128 & 108 & 100 & $\underline{195}$ & 135 & 11.233 & $\underline{135}$ & 116 \\
\hline Average 27-32 Well & & & 78 & 108 & 244 & 213 & 134 & 151 & 129 & $\overline{197}$ & & & $\overline{167}$ & \\
\hline
\end{tabular}

"Calculations use the following geothermometers: $\mathrm{tCH}, \mathrm{tQC}$, and $\mathrm{tNK}(\mathrm{f})=$ the chalcedony, quartz (conductive) and $\mathrm{Na} / \mathrm{K}$ equations of Fournier $(1981)$; $\mathrm{tNL}(\mathrm{d})=\mathrm{the} \mathrm{Na} / \mathrm{Li}(\mathrm{dilute})$ equation of Fouillac and Michard (1981); tL(d) and $\mathrm{tML}=$ the $\mathrm{Li}$ (dilute) and Mg/Li equations of Kharaka and Mariner (1989); tKM = the K/Mg equation of Giggenbach (1986); Na-K-Ca refers to the equations of Fournier and Truesdell, 1973 (see their paper for an explanation of the "beta" factor); Na-K-Ca Mg correction refers to the equations of Fournier and Potter, 1979 ("cool" means $<70{ }^{\circ} \mathrm{C}$ )

"Values in bold use assumed concentrations of $0.01 \mathrm{ppm} \mathrm{Mg}$ to perform the calculation (see Table 10).

"The underlined value is the preferred temperature according to the rules of the geothermometer. 
Table 12: Chemical Geothermometry of Background Thermal/Mineral Springs and Wells."

\begin{tabular}{|c|c|c|c|c|c|c|c|c|c|c|c|c|c|c|c|}
\hline \multirow{3}{*}{$\begin{array}{l}\text { Sample } \\
\text { Springs }\end{array}$} & \multirow{2}{*}{ Name or Description } & \multirow[t]{2}{*}{ Date } & \multirow{3}{*}{$\begin{array}{l}\text { Sampling } \\
\text { Temp }\left({ }^{\circ} \mathbf{C}\right)\end{array}$} & \multirow[t]{2}{*}{$\mathrm{tCH}$} & \multirow[t]{2}{*}{ tQC } & \multirow[t]{3}{*}{$\operatorname{tNK}(\mathbf{f})$} & \multirow[t]{3}{*}{$\mathbf{t N L}(\mathbf{d})$} & \multirow[t]{3}{*}{$\mathbf{t L}(\mathbf{d})$} & \multirow[t]{3}{*}{ tML } & \multirow[t]{2}{*}{ tKM } & \multicolumn{5}{|c|}{ <------ Na-K-Ca------><---- Na-K-Ca Mg correction ----> } \\
\hline & & & & & & & & & & & & & $\mathbf{R}$ & & \\
\hline & & & & & & & & & & & & & & & \\
\hline DV97-46 & Sou Hot Spring & $11 / 03 / 97$ & 57.0 & 85 & 114 & $27 \mathrm{I}$ & 180 & 145 & 75 & 84 & $\underline{196}$ & 104 & 22 & $\underline{83}$ & 80 \\
\hline DV97-47 & Sou Hot Spring & $11 / 03 / 97$ & 72.6 & 83 & 113 & 270 & 179 & 143 & 74 & 83 & $\underline{195}$ & 102 & 21 & $\underline{86}$ & 82 \\
\hline DV $98-117^{\circ}$ & Sou Hot Spring & $05 / 04 / 98$ & 72.0 & 80 & 109 & 264 & -29 & 37 & -14 & 84 & 193 & 104 & 22 & $\underline{83}$ & 80 \\
\hline DV97-48 & Hyder Hot Spring & $11 / 03 / 97$ & 76.7 & 81 & 111 & 180 & 194 & 174 & 112 & 84 & $\underline{161}$ & 124 & 24 & $\underline{71}$ & 71 \\
\hline DV98-112 & Hyder Hot Spring & $04 / 30 / 98$ & 75.3 & 79 & 109 & 174 & 179 & 171 & 109 & 84 & $\underline{158}$ & 125 & 24 & $\underline{72}$ & 72 \\
\hline DV97-56 & Dead Travertine Spring, upper seep & $11 / 05 / 97$ & 17.4 & 44 & 76 & 254 & 211 & 181 & 85 & 80 & $\underline{194}$ & 116 & 37 & $\underline{39}$ & 43 \\
\hline DV99-210 & Dead Travertine Spring, road seep & $05 / 07 / 99$ & $19-22$ & 50 & 82 & 256 & 210 & 185 & 86 & 82 & 200 & 131 & 44 & $\underline{25}$ & 27 \\
\hline DV97-60 & Fault Line Spring & $11 / 06 / 97$ & 28.8 & 62 & 93 & 192 & 125 & 121 & 57 & 64 & 154 & 83 & 29 & 55 & $\underline{68}$ \\
\hline DV97-61 & Lower Ranch Hot Spring & $11 / 06 / 97$ & 40.8 & 62 & 92 & 199 & 117 & 114 & 55 & 67 & 161 & $\underline{93}$ & 33 & 45 & $\underline{56}$ \\
\hline DV98-113 & Lower Ranch Hot Spring & $05 / 04 / 98$ & 40.4 & 56 & 87 & 191 & 110 & 114 & 55 & 67 & 157 & 94 & 33 & 46 & $\underline{56}$ \\
\hline DV99-211 & Lower Ranch, upper spring & $05 / 08 / 99$ & 39.4 & 59 & 90 & 200 & 111 & 113 & 54 & 68 & 161 & $\underline{95}$ & 32 & 48 & $\underline{58}$ \\
\hline DV97-62 & McCoy Hot Spring & $11 / 06 / 97$ & 46.2 & 55 & 87 & 163 & 69 & 98 & 33 & 53 & 136 & $\underline{72}$ & 37 & 39 & $\underline{57}$ \\
\hline DV98-114 & McCoy Hot Spring & $05 / 04 / 98$ & 46.0 & 51 & 82 & 156 & 70 & 102 & 35 & 53 & 133 & $\underline{73}$ & 37 & 39 & $\underline{56}$ \\
\hline DV97-63 & Kyle Spring & $11 / 06 / 97$ & 19.8 & 25 & 58 & 126 & 14 & 50 & -8 & 24 & 101 & $\underline{26}$ & 43 & 37 & $\underline{26}$ \\
\hline DV97-68 & Big Horn Spring & $11 / 07 / 97$ & 20.5 & 56 & 88 & 79 & -4 & 74 & 9 & 35 & 87 & $\underline{58}$ & 47 & 36 & $\underline{58}$ \\
\hline DV98-118 & Big Horn Spring & $05 / 05 / 98$ & 18.1 & 53 & 84 & 69 & -14 & 81 & 11 & 39 & 84 & $\underline{68}$ & 46 & 37 & 68 \\
\hline DV97-69 & Dixie Hot Spring & $11 / 07 / 97$ & 81.6 & 113 & 140 & 122 & 123 & 126 & 141 & 106 & 123 & $\underline{97}$ & 1 & 123 & $\underline{97}$ \\
\hline DV98-120 & Dixie Hot Spring & $05 / 05 / 98$ & 83.5 & 114 & 141 & 117 & 117 & 126 & 130 & 97 & 120 & $\underline{98}$ & 3 & 120 & $\underline{98}$ \\
\hline DV98-128 & Jersey Hot Spring & $05 / 05 / 98$ & 59.0 & 129 & 154 & 211 & 208 & 159 & 117 & 96 & $\underline{177}$ & 126 & 14 & $\underline{115}$ & 105 \\
\hline \multicolumn{16}{|l|}{ Wells } \\
\hline DV96-1 & Domestic Well & $10 / 24 / 96$ & 34.2 & 93 & 121 & 223 & 147 & 127 & 55 & 64 & 171 & 93 & 43 & 26 & $\underline{39}$ \\
\hline DV97-38 & Domestic Well & $10 / 24 / 96$ & 29.2 & 95 & 123 & 233 & 149 & 126 & 56 & 66 & 177 & $\underline{95}$ & 41 & 29 & $\underline{40}$ \\
\hline DV97-39 & Goerenger Well & $10 / 31 / 97$ & 27.8 & 83 & 112 & 208 & 169 & 151 & 72 & 69 & 172 & 114 & 50 & $\mathrm{CoOl}$ & Cool \\
\hline DV98-160 & Goerenger Well & $10 / 23 / 98$ & 26.7 & 81 & 110 & 207 & 158 & 147 & 67 & 67 & 171 & 113 & 54 & Cool & Cool \\
\hline DV98-96 & Goerenger Well & $04 / 29 / 98$ & 28.3 & 82 & 112 & 205 & 161 & 151 & 71 & 69 & 172 & 117 & 52 & Cool & Cool \\
\hline DV99-181 & Goerenger Well & $05 / 04 / 99$ & 27.7 & 82 & 111 & 198 & 159 & 152 & 70 & 67 & 167 & 114 & 54 & $\mathrm{CoOl}$ & $\mathrm{Cool}$ \\
\hline DV97-57 & Bolivia Artesian Well & $11 / 05 / 97$ & 28.8 & 42 & 74 & 127 & 81 & 98 & 25 & 30 & 107 & $\underline{42}$ & 52 & Cool & $\underline{42}$ \\
\hline DV97-59 & 45-W-5 Well & $11 / 05 / 97$ & 26.4 & 36 & 68 & 152 & 155 & 141 & 101 & 78 & $\underline{151}$ & 141 & 35 & $\underline{42}$ & 42 \\
\hline DV97-67 & 66-21 Well & $11 / 07 / 97$ & 55.5 & 199 & 215 & 210 & 188 & 215 & 225 & 182 & $\underline{202}$ & 216 & 1 & $\underline{200}$ & 215 \\
\hline DV98-104 & 66-21 Well & $04 / 30 / 98$ & 57.4 & 200 & 216 & 216 & 200 & 219 & 233 & 185 & $\underline{205}$ & 216 & 1 & $\underline{202}$ & 214 \\
\hline DV98-111 & $62-21$ Well & $04 / 30 / 98$ & 75.5 & 147 & 170 & 138 & 74 & 131 & 124 & 124 & 153 & 184 & 4 & $\underline{151}$ & 171 \\
\hline DV98-122 & $97-2$ Well & $05 / 05 / 98$ & 19.7 & 77 & 106 & 210 & 165 & 168 & 82 & 79 & 182 & 143 & 53 & Cool & $\mathrm{Cool}$ \\
\hline DV98-123 & $32-6$ Well & $05 / 06 / 98$ & 32 & -33 & 0 & 146 & 33 & 78 & 9 & 40 & 132 & $\underline{85}$ & 77 & $\mathrm{Cool}$ & $\mathrm{Cool}$ \\
\hline DV98-168 & $38-32$ Well & $10 / 26 / 98$ & 87.7 & 144 & 168 & 223 & 174 & 154 & 131 & 121 & $\underline{194}$ & 161 & 6 & $\underline{167}$ & 148 \\
\hline DJ-1 & Dixie Jack Gradient Well \#I & $05 / 17 / 98$ & 49 & 64 & 95 & 181 & 170 & 173 & 121 & 99 & $\underline{166}$ & 140 & 15 & $\underline{108}$ & 103 \\
\hline DJ -4 & Dixie Jack Gradient Well \#4 & $05 / 20 / 98$ & 77 & 121 & 147 & 206 & 173 & 160 & 151 & 131 & $\underline{187}$ & 168 & 4 & 178 & 164 \\
\hline DJ -7 & Dixie Jack Gradient Well \#7 & $05 / 14 / 98$ & 55 & -15 & 18 & 222 & 181 & 84 & 82 & 78 & 160 & $\underline{65}$ & 5 & 151 & $\underline{65}$ \\
\hline
\end{tabular}

"Calculations use the geothermometers described on the bottom of Table 11.

"The underlined value is the preferred temperature according to the rules of the geothermometer.

"This sample assumes $0.01 \mathrm{ppm}$ lithium to perform the genthermometer calculations shown in bold 
Table 13: Chemical Analyses of Scales and Precipitates from Dixie Valley Production Wells, Pipelines, and Test Beds. ${ }^{a}$

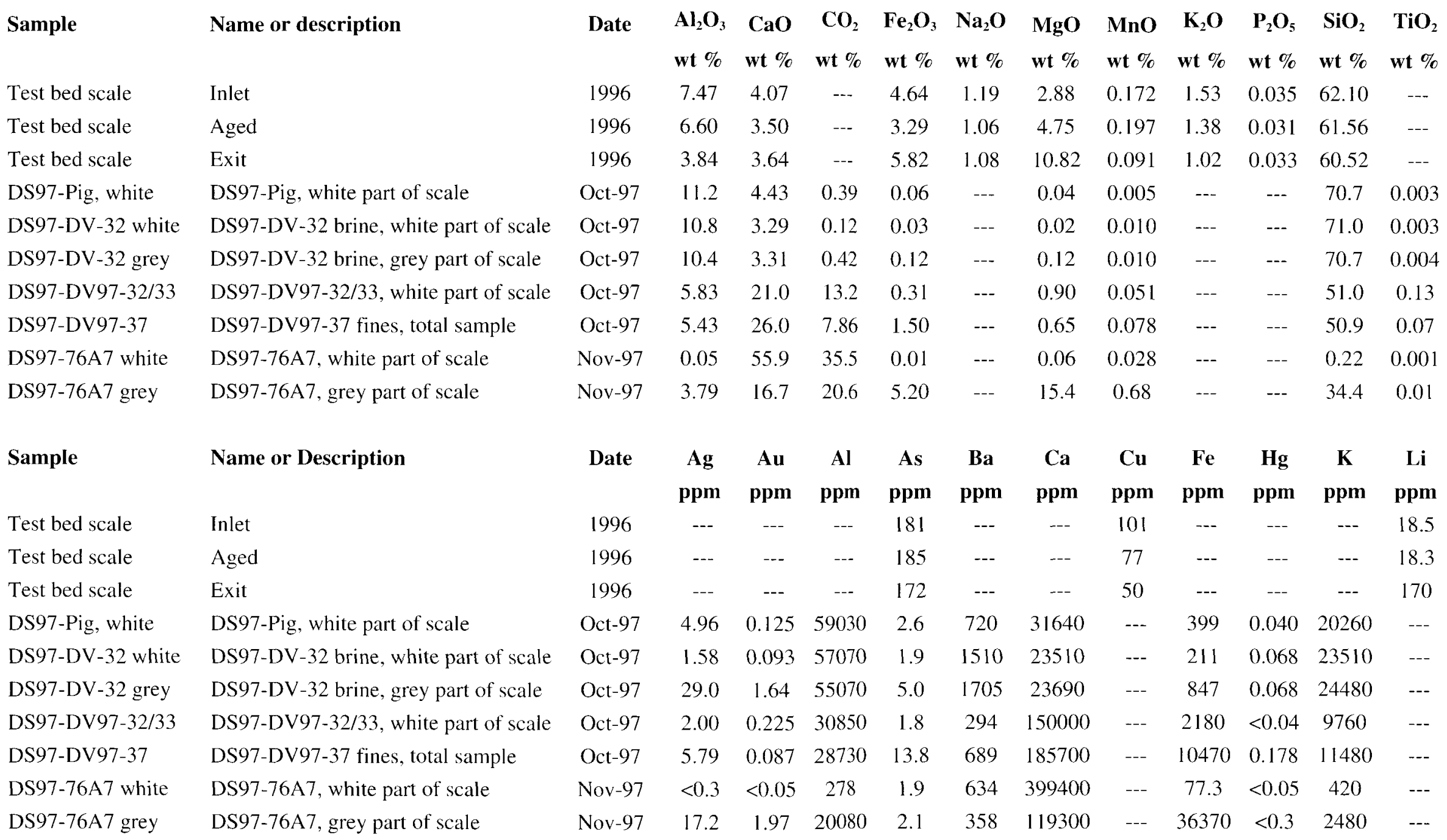

"Data for the test-bed scale was previously published in Bruton et al. (1997). 
Table 13: Continued

\begin{tabular}{|c|c|c|c|c|c|c|c|c|c|}
\hline Sample & $\begin{array}{c}\text { Cl } \\
\text { wt } \%\end{array}$ & $\begin{array}{c}F \\
\text { wt } \%\end{array}$ & $\begin{array}{c}S \\
\text { wt } \%\end{array}$ & $\begin{array}{r}\text { TIC } \\
\text { wt \% }\end{array}$ & $\begin{array}{l}\text { TOC } \\
\text { wt \% }\end{array}$ & & & & \\
\hline Test bed scale & 0.113 & 0.033 & 0.136 & 0.083 & 0.272 & & & & \\
\hline Test bed scale & 0.058 & 0.067 & 0.130 & 0.065 & 0.172 & & & & \\
\hline Test bed scale & 0.121 & 0.183 & 0.109 & 0.282 & 0.180 & & & & \\
\hline DS97-Pig, white & --- & --- & -- & --- & -- & & & & \\
\hline DS97-DV-32 white & --- & --- & --- & --- & --- & & & & \\
\hline DS97-DV-32 grey & --- & --- & --- & --- & -- & & & & \\
\hline DS97-DV97-32/33 & --- & --- & --- & --- & --- & & & & \\
\hline DS97-DV97-37 & $\cdots$ & -- & -- & --- & --- & & & & \\
\hline DS97-76A7 white & $-\cdots$ & --- & --- & --- & --- & & & & \\
\hline DS97-76A7 grey & --- & --- & --- & --- & --- & & & & \\
\hline Sample & $\begin{array}{c}\mathrm{Mg} \\
\mathbf{p p m}\end{array}$ & $\begin{array}{c}\text { Mn } \\
\text { ppm }\end{array}$ & $\begin{array}{c}\text { Mo } \\
\text { ppm }\end{array}$ & $\begin{array}{c}\mathrm{Na} \\
\mathrm{ppm}\end{array}$ & $\begin{array}{c}\mathrm{Ni} \\
\mathrm{ppm}\end{array}$ & $\begin{array}{c}\mathbf{S i} \\
\mathbf{p p m}\end{array}$ & $\begin{array}{c}\text { Sr } \\
\text { ppm }\end{array}$ & $\begin{array}{c}\mathrm{Ti} \\
\mathbf{p p m}\end{array}$ & $\begin{array}{l}\text { TIC } \\
\text { ppm }\end{array}$ \\
\hline Test bed scale & --- & --- & 4.0 & --- & 20.5 & -.- & 1180 & --- & --- \\
\hline Test bed scale & --- & --- & 6.1 & --- & 9.4 & -- & 2070 & --- & --- \\
\hline Test bed scale & --- & --- & $<4$ & --- & 32.3 & --- & 7 & --- & --- \\
\hline DS97-Pig, white & 250 & 38.7 & -- & 8020 & --- & 330400 & 2920 & 19.8 & 1060 \\
\hline DS97-DV-32 white & 144 & 74.6 & --- & 9300 & -- & 332000 & 1940 & 18.1 & 333 \\
\hline DS97-DV-32 grey & 743 & 74.3 & -- & 9810 & --- & 330400 & 1950 & 24.8 & 1160 \\
\hline DS97-DV97-32/33 & 5460 & 394 & --- & 5410 & --- & 238200 & 1570 & 801 & 36000 \\
\hline DS97-DV97-37 & 3900 & 608 & --- & 5150 & -- & 238100 & 939 & 421 & 21440 \\
\hline DS97-76A7 white & 344 & 214 & -- & 151 & -- & 1050 & 8440 & 7.6 & 96900 \\
\hline DS97-76A7 grey & 92840 & 5300 & --- & 5670 & --- & 160600 & 2980 & 73.9 & 56060 \\
\hline
\end{tabular}


Table 14: Miscellaneous Isotope Data for Vein and Rock Samples, Dixie Valley Region, Nevada."

\section{Sample Name or Description \\ Location}

F97-22 Marine limestone, post Star Peak strata

F98-8 Calcite vein in graphitic argillite

F98-9 Gypsum in quartzile fault gouge beneath gabbro

F98-10 Graphitic marble, Stillwater Range

F98-12 Calcite-quartz veins in altered quarzite, Stillwater Range Abour $0.25 \mathrm{~km} \mathrm{NW}$ of Senator Fumarole

F98-13 Calcite vein in gabbro

F98-14 Calcite crystals in fault gouge,

F98-15 Sulphur from Range Front Fumarole

F98-16 Pyrite in quartzite breccia

F98-17 Sulphur from Senator Fumarole

F98-18 Coarse-grained diorite (part of Humboldt Lopolith?)

F98-19 Welded tuff, overlies diorite

F98-20 Welded iff, undeties pyroclasic falla

F98-40 Meta-argillite, occurs near quartzite, Bernice Formation Clan Alpine Range, Lofthousc Canyon (spg DV98-170)

F98-44 Calcareous argillite. Hoyt Canyon Formation Clan Alpine Range, Hoyt Canyon (crk DV98-174)

F98-45 Quartzite, overlies argillite, Hoyt Canyon Formation

Clan Alpine Range, Hoyt Canyon (crk DV98-174)

F98-48 Medium-orained diorite, underlies quartzite, Imst, and tuff Clan Alpine Range, $16 \mathrm{~km}$ NW of Shoshone Pass

F98-49 Marine limestone, underlies welded tuff

D F98-50 Welded tuff, slightly altered and silicified

F98-52 Sheared marine limestone, Star Peak Group

F98-54 Gabbro-diorite, underies thrust fault and quartzitc

Clan Alpine Range, $10 \mathrm{~km}$ NW of Shoshone Pas

Clan Alpine Range, War Canyon (near spg DV98-176)

Stillwater fault zone at Figure 8 Fumarole

\section{Samples from Dixie Valley region}

Stillwater Range, Cotonwood Canyon, W of travertine

Age

$\delta^{13} \mathbf{C}(\mathbf{P D B}) \delta^{18} \mathrm{O}$ (SMOW) $\delta^{\mathrm{w}} \mathrm{S}(\mathrm{CDT}) \quad \mathbf{R b}$ (per mil)

$\begin{array}{lll}\text { U. Triassic } & 1.37 & 16.6\end{array}$

Quaternary $\quad-3.9$

Quters

Jurassic

Quaternary

Quaternary

Quaternary

1998

Quaternary

1998

Jurassic

Oligocene

Oligocene

U. Triassic

U. Triassic

U. Triassic

Jurassic

U. Triassic

U. Triassic

M. Triassic

Jurassic

\section{Samples from North Central Nevada}

F92-42 Marine dolomite, Nevada Group

F92-43 Vinini Group chert

F92-44 Vinini Group shale

F92-45 Travertine deposit at Buffy Hot Spring

F92-46 Troy Canyon muscovite granite

F92-48 Troy Canyon ignimbrite

F92-49 Goodwyn Canyon Dolomite

F92-50 Chainman Shale

F92-51 Guilmette Limestone

F92-52 Joana Limestone

F92-53 Chainman Shale

F92-54 Pancake Range ignimbrit

F92-55 Stone Cabin ignimbrite

F92-56 Horse Heaven ignimbrite

F92-57 Sheep Pass Formation, organic-rich lacustrine beds
Sulphur Springs Range, east of Pine Valley

Sulphur Springs Range. east of Pine Valley

Sulphur Springs Range, east of Pine Valley

Sulphur Springs Range, east of Pine Valley

Grant Range, cast of Railroad Valley

Grant Range, east of Railroad Valley

Grant Range, east of Railroad Valley

Grant Range. east of Railroad Valley

Grant Range, east of Railroad Valley

Grant Range, east of Railroad Valley

Pancake Range, west of Railroad Valley

Pancake Range, west of Railroad Valley

Pancake Range, west of Railroad Valley

Pancake Range, west of Railroad Valley

Grant Rangc, cast of Railroad Valley

Devonian
Ordovician
Ordovician
Holocene
Cretaceous
Oligocene
Ordovician
Mississippian
Devonian
Mississippian
Mississippian
Oligocene
Oligocene
Oligocene

1.3
-3.
-
1.3
-4.
-4
-3.
-
-
-
-
-
-
-
-
-
-
-
-
-

1.3

$-4.3$

$-4.0$

$---$

$---$ (per mil)

(per mil)

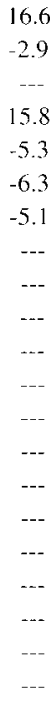

$-2.9$

15.8

$\begin{array}{cc}--. & - \\ --. & - \\ 0.2 & \\ -- & < \\ --- & - \\ --- & - \\ --- & - \\ 4.6 & - \\ 6.0 & \\ 2.6 & - \\ --- & 56.8 \\ --- & 105 \\ --- & 127 \\ --- & 85 \\ --- & 32 \\ --- & 25 \\ --- & 8.1 \\ --- & < \\ --- & 7.8 \\ --- & 35.9 \\ --- & - \\ & \end{array}$

---
$\cdots-$
$\cdots-$
$<1$
$\cdots-$
$\cdots-$
$\cdots-$
--
--
$\cdots-$
56.8
105
127
85.2
32.2
25.3
8.1
$<1$
7.8
35.9
.--

Sr Laboratory

---
---
---
---
---
---
--
--
---
--
---
---
---
---
---

\begin{tabular}{lr}
--- & 5.0 \\
--- & 0.56 \\
--- & 4.0 \\
--- & 1 \\
--- & 100.4 \\
--- & 294 \\
-- & 4.7 \\
--- & 86 \\
--- & 0.77 \\
-- & 0.55 \\
--- & 78.1 \\
--- & 128 \\
--- & 129 \\
--- & 261 \\
\hline- & 1.81
\end{tabular}

5.0
0.56
4.0
11.4
100.3
294
4.7
86
0.77
0.55
78.1
128
129
261
1.81

385
6.96
53.3
86.8
475
1.32
551
178
22.8
194
242
340
372
340
969

\begin{tabular}{|c|c|}
\hline-- & USGS \\
\hline -- & UNM \\
\hline--- & $\mathrm{GC}$ \\
\hline 0.707926 & UNM/LAN \\
\hline -.. & UNM \\
\hline -- & UNM \\
\hline --. & UNM \\
\hline -- & $\mathrm{GC}$ \\
\hline --- & $\mathrm{GC}$ \\
\hline --- & GC \\
\hline 0.704710 & UNM/LAN \\
\hline 0.710082 & UNM/LAN \\
\hline 0.708330 & UNM/LAN \\
\hline 0.720839 & UNM/LAN \\
\hline 0.708686 & UNM/LAN \\
\hline 0.718175 & UNM/LAN \\
\hline 0.705198 & UNM/LAN \\
\hline 0.708007 & UNM/LAN \\
\hline 0.706148 & UNM/LAN \\
\hline 0.707749 & UNM/LAN \\
\hline 0.705409 & UNM/LAN \\
\hline 0.708658 & UNM \\
\hline 0.715335 & UNM \\
\hline 0.714235 & UNM \\
\hline 0.712022 & UNM \\
\hline 0.714216 & UNM \\
\hline 0.711504 & UNM \\
\hline 0.711282 & UNM \\
\hline 0.717174 & UNM \\
\hline 0.709684 & UNM \\
\hline 0.708406 & UNM \\
\hline 0.716672 & UNM \\
\hline 0.712557 & UNM \\
\hline 0.712320 & UNM \\
\hline 0.710694 & UNM \\
\hline
\end{tabular}

"Stable isotope analyses were performed at Geochron L aboratories, Cumbridge, Masuchusets. Strontium isotope analyses were obtained from the University of New Mexico, Albuquerque, New Mexico. "Collection years for all samples are in the sample number, i.e., F97-22 was collected in 1997.

'Many samples were identified using the geologic map of Speed (1976) and the report of Lutz et al (1997)

'This group of samples was collected for an earlier geothermal project but data were unpublished (Goff et al., 1994 and Hulen et al.. 1994). 
Table 15: Uranium-Thorium and Uranium-Protactinium Dates on Two Samples of Old Spring Deposits, Dixie Valley, Nevada

\begin{tabular}{|c|c|c|}
\hline Sample No. & F99-50b & F99-61 \\
\hline Material & Travertine Vein & Sinter Layer \\
\hline Location & Cottonwood Canyon & Lower Ranch \\
\hline Weight $(g)$ & 1.6666 & 0.9924 \\
\hline Th (ppm) & 0.00443 & 0.04653 \\
\hline$(+/-, \%)$ & 0.36 & 0.85 \\
\hline $\mathrm{U}(\mathrm{ppm})$ & 0.71 & 0.04 \\
\hline$(+/-, \%)$ & 0.25 & 0.31 \\
\hline $\mathrm{Th} / \mathrm{U}$ & 0.0062 & 1.1691 \\
\hline$(+/-, \%)$ & 0.44 & 0.91 \\
\hline${ }^{234} \mathrm{U} /{ }^{238} \mathrm{U}$ & 1.598 & 1.245 \\
\hline${ }^{230} \mathbf{T h} /{ }^{234} \mathrm{U}$ Age $(\mathbf{k a})$ & $182 \pm 4$ & $54 \pm 4$ \\
\hline${ }^{231} \mathrm{~Pa} /{ }^{235} \mathrm{U}$ & 0.967 & 0.565 \\
\hline$(+/-, \%)$ & 0.74 & 1.05 \\
\hline${ }^{231} \mathbf{P a} /{ }^{235} \mathbf{U}$ Age (ka) & $161 \pm 15$ & $39 \pm 2$ \\
\hline Initial ${ }^{234} \mathrm{U} /{ }^{238} \mathrm{U}$ & 1.842 & 1.344 \\
\hline
\end{tabular}

"Honey-colored calcite vein cutting altered gabbro, base of spring deposit near seep sample DV97-56.

'Sinter separate from layered silica and carbonate, base of northwestern edge of deposit, about $0.3 \mathrm{~km}$ from hot spring DV97-61. 
This report has been reproduced directly from the best available copy. It is available electronically on the Web (http://www.doe.gov/bridge).

Copies are available for sale to U.S. Department of Energy employees and contractors from:

Office of Scientific and Technical Information

P.O. Box 62

Oak Ridge, TN 37831

(865) $576-8401$

Copies are available for sale to the public from: National Technical Information Service

U.S. Department of Commerce

5285 Port Royal Road

Springfield, VA 22616

(800) 553-6847 
- Los Alamos

Los Alamos NM 87545 